\title{
Design and evaluation of CCA (Cooperative Collision Avoidance) applications for Vehicular Ad-hoc Networks
}

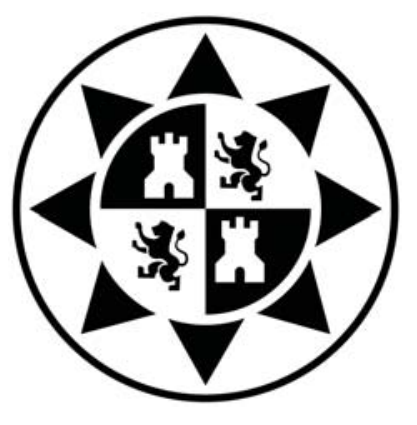

Juan Bautista Tomás Gabarrón

Departamento de Tecnologías de la Información y las Comunicaciones Universidad Politécnica de Cartagena

A thesis submitted for the degree of Dr.-Ing. (PhD degree in Engineering) 


\section{Design and evaluation of CCA (Cooperative Collision Avoidance) applications for Vehicular Ad-hoc Networks}

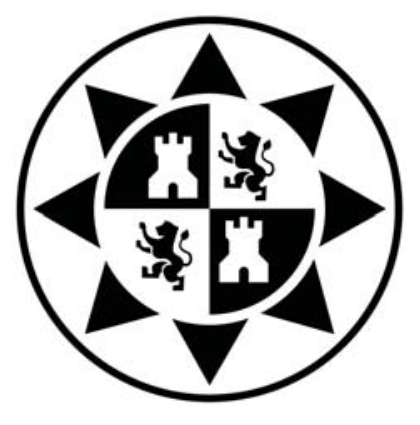

Juan Bautista Tomás Gabarrón

Departamento de Tecnologías de la Información y las Comunicaciones Universidad Politécnica de Cartagena

A Thesis directed and supervised by:

Prof. Joan García Haro, PhD

Universidad Politécnica de Cartagena 


\section{CONFORMIDAD DE SOLICITUD DEAUTORIZACIÓN DE DEPÓSITO DE} TESIS DOCTORAL POR EL/LA DIRECTOR/A DE LA TESIS

D./Da Joan García Haro Director/a de la Tesis doctoral "Design and evaluation of CCA (Cooperative Collision Avoidance) applications for Vehicular Ad-hoc Networks "

\section{INFORMA:}

Que la referida Tesis Doctoral, ha sido realizada por D/Da Juan Bautista Tomás Gabarrón, dando mi conformidad para que sea presentada ante la Comisión de Doctorado, para ser autorizado su depósito.

La rama de conocimiento por la que esta tesis ha sido desarrollada es:

$\square \quad$ Ciencias

$\square$ Ciencias Sociales y Jurídicas

$\mathrm{X}$ Ingeniería y Arquitectura

En Cartagena, a 8 de Enero de 2013

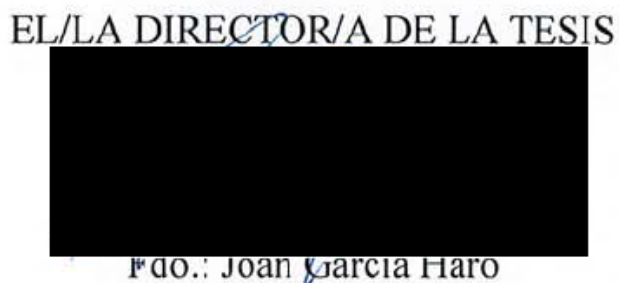

COMISIÓN DE DOCTORADO 


\section{CONFORMIDAD DE DEPÓSITO DE TESIS DOCTORAL POR LA COMISIÓN ACADÉMICA DEL PROGRAMA}

D/Da. Fernando Daniel Quesada Pereira, Presidente/a de la Comisión Académica del

Programa Tecnologías de la Información y las Comunicaciones (TIC).

\section{INFORMA:}

Que la Tesis Doctoral titulada "Design and evaluation of CCA (Cooperative Collision Avoidance) applications for Vehicular Ad-hoc Networks", ha sido realizada por D/D .Juan Bautista Tomás Gabarrón,

bajo la dirección y supervisión del Dr/ Dra. Joan García Haro.

En reunión de la Comisión Académica de fecha $10 / 12 / 12$, visto que la mencionada tesis doctoral tiene acreditados los indicios de calidad, requeridos para el depósito de tesis doctorales, regulados en el artículo 32 del Reglamento de Estudios Oficiales de Máster y Doctorado de la UPCT, y la autorización del Director de la misma, se acordó dar la conformidad para que a dicha tesis le sea autorizado, por la Comisión de Doctorado, su depósito.

La Rama de conocimiento por la que esta tesis ha sido desarrollada es:

Ciencias

$\square$ Ciencias Sociales y Jurídicas

$\mathrm{X}$ Ingeniería y Arquitectura

En Cartagena, a 8 de Enero de 2013

SOd NOIO

EL PRESIDENTE DE LA COMNSTA AEAREAICA DEL PROGRAMA

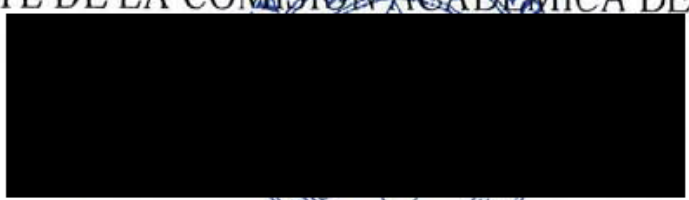

Fdo: Fernando anies 
A mis amigos y amigas, porque ellos son un verdadero tesoro, aquél que de verdad merece la pena cuidar en esta vida.

A mis padres, Juan e Isabel, a quiénes les debo la vida, quiénes siempre me han ayudado, quiénes siempre han puesto por delante el amor a sus

hijos frente a cualquier otra circunstancia, y a quiénes nunca podré pagarles todo lo que me han dado. A mi hermano José Ángel, por ser mi compañero durante los años de mi infancia, por ser una persona leal y excepcional, por ser alguien que siempre está ahí.

Y a Marina, porque eres la luz que alumbra mi vida. Gracias por tener paciencia conmigo, cada día, por perdonarme cuando cometo errores, por apoyarme cuando las cosas me salen bien, por ser la guía que cada día me enseña algo nuevo. Te quiero.

Gracias de corazón 


\begin{abstract}
New emerging technologies in vehicular traffic are aimed primarily at improving safety and driving comfort for passengers, by paying special attention to the gradual evermore automation of all aspects of the driving task. In this regard, a promising research perspective considered by the Academia and the Industry is to use communications to build a complex interoperable vehicular network that would serve as a means to provide autonomous robotic-guided vehicles with additional status information that might not be collected from sensors on board. With properly configured processing schemes, this additional stream of information can be used to help vehicles anticipate and react conveniently to potentially risky situations that might cause an accident if not previously considered. Particularly, in this Thesis we use these premises to propose and evaluate collision avoidance policies under two specific fashions: i) Design and evaluation of a Cooperative chain Collision Avoidance $(\mathrm{CcCA})^{1}$ strategy to reduce the impact of multiple rear-end collisions in a platoon of vehicles when evasive maneuvering is not possible, and ii) Analysis and optimization of different strategies for Cooperative Collision Avoidance (CCA) by evasive maneuvering. The CcCA application allows us to study how communication protocols, both by one-hop transmissions as well as by relaying (multi-hop) schemes, can help reduce the number of accidents, or at least minimize their impact, in cases where vehicles cannot execute sudden maneuvers to skip cars ahead, but only brake. Simulations are validated by using an advanced stochastic model which rigorously describes the behavior of vehicles in this type of situations. Among other aspects, results show that real implementations of CcCA must take into account with special relevance those vehicles that
\end{abstract}

${ }^{1}$ That we denote as $\mathrm{CcCA}$ to distinguish from general Cooperative Collision Avoidance (CCA) applications. 
might be humanly driven, and guarantee that during the transition stage (until a complete penetration of the technology is achieved) safety is preserved enough. Regarding CCA for evasive maneuvering, we provide an exhaustive optimization analysis for the calculation of optimum trajectories in cases where vehicles at high speeds are at risk of colliding with one or more obstacles appearing ahead. By reorienting trajectories through the lateral free spaces that might exist between the obstacles and the crash barriers (if the specific scenario allows it), vehicles can avoid crashing and simultaneously improve driving comfort even under such unpredictable circumstances. On the whole, despite much further effort is still required on these matters, results in this Work show that communications can help autonomous vehicles to make decisions in a cooperative fashion that will not only assist individuals to follow the best riding strategy, but also the traffic system as a whole to evolve according to the best possible behavior in terms of safety and comfort. 


\section{Resumen}

Las nuevas tecnologías emergentes en el tráfico rodado están destinadas principalmente a mejorar la seguridad y el confort de los ocupantes, con especial hincapié en la progresiva automatización de todos los elementos que conciernen la conducción. En este sentido, una perspectiva de investigación prometedora (así considerado por los OPIs y la Industria) es utilizar las comunicaciones para construir una compleja red vehicular interoperable que serviría como un medio para proporcionar a vehículos auto-guiados información de estado adicional (principalmente sobre localización y posicionamiento) que podría no ser obtenida por los sensores de a bordo. Por medio de dispositivos de procesamiento configurados a tal efecto, este flujo adicional de datos podría ser utilizado para ayudar a los vehículos a anticipar de forma efectiva situaciones potencialmente peligrosas que podrían ser causantes de un accidente de consecuencias imprevisibles. En particular, en este trabajo se aborda la propuesta y evaluación de políticas de prevención de colisiones bajo dos premisas específicas: i) Diseño y evaluación de una aplicación para la reducción del número de accidentes en cadena $(\mathrm{CcCA})^{1}$, fundamentada en la propuesta de estrategias para reducir el impacto de múltiples colisiones por alcance en un convoy de vehículos cuando la ejecución de maniobras evasivas no es posible, y ii) Análisis y optimización de estrategias de prevención de colisiones de forma cooperativa (CCA, Cooperative Collision Avoidance) mediante maniobras de evasión. La aplicación CcCA nos permite estudiar cómo los protocolos de comunicación, tanto de tipo mono-salto como aquellos basados en esquemas multi-salto, nos pueden ayudar a reducir el número de accidentes, o por lo menos minimizar su impacto, en aquellos casos en los que los vehículos no pueden ejecutar

\footnotetext{
${ }^{1}$ Esto se denota como CcCA, (Cooperative chain Collision Avoidance), véase Abstract en inglés.
} 
cambios de dirección para evitar colisiones frontales, y sólo pueden recurrir a frenadas bruscas en la dirección en la que se circula. Las simulaciones realizadas han sido validadas mediante el uso de un modelo estocástico avanzado que describe rigurosamente el comportamiento de los vehículos en este tipo de escenarios. Entre otros aspectos, los resultados muestran que en escenarios reales donde existan vehículos que implementen $\mathrm{CcCA}$, se habrá de tener en cuenta con especial atención la interacción entre vehículos autónomos y los asistidos por conductores humanos, y garantizar del mismo modo que durante la etapa de transición (hasta que se logre una completa penetración de la tecnología en el mercado) se preserve la seguridad suficientemente. En cuanto a CCA para maniobras de evasión, se proporciona un análisis exhaustivo para el cálculo de trayectorias óptimas en el caso de vehículos circulando a altas velocidades que se hallan en riesgo de chocar con uno o más obstáculos que aparecen en la dirección de tránsito. Mediante la reorientación de las trayectorias hacia los espacios laterales libres que podrían existir entre los obstáculos y las barreras laterales de la carretera (si la situación así lo permite), los vehículos tendrían la posibilidad de evitar colisionar y al mismo tiempo reducir el impacto inercial de la maniobra sobre los pasajeros bajo estas circunstancias. En general, a pesar de que todavía se requiere un esfuerzo más continuado en la investigacion de estos asuntos, los resultados de este trabajo muestran que las comunicaciones pueden ayudar a los vehículos autónomos a tomar decisiones de forma cooperativa que no sólo servirán para seguir la mejor y más segura estrategia de conducción en condiciones particulares de riesgo extremo, sino que también serán de enorme utilidad para el sistema del tráfico en su conjunto de cara a proporcionar altas cotas de seguridad y comodidad bajo cualquier circunstancia. 


\section{Acknowledgements}

This Thesis was supported by project grants MICINN/FEDER TEC201021405-C02-02/TCM (CALM) and 00002/CS/08 FORMA (Fundación SénecaBen Arabi Supercomputer RM). It was also developed in the framework of 'Programa de Ayudas a Grupos de Excelencia de la Región de Murcia, Fundación Séneca'. J. B. Tomas-Gabarron also thanks the Spanish MICINN for a FPU (REF AP2008-02244) pre-doctoral fellowship.

At first place, the Author would like to thank every single person of my country, Spain, who during the current dramatic world crisis that has even impacted on our economy with a much virulent force, has contributed with his taxes to pay the salary with which I could accomplish my Thesis. Thank you.

The Author would also like to personally thank PhD. candidate Carolina García Costa for her suggestions, aids and support along the predoctoral phase, which have been indispensable for the completion of this Thesis.

The Doctorand would also like to acknowledge Dr.-Ing. Esteban Egea for his devoted effort during the last four years to propose improvements, revise the work, provide new ideas, and, in general, because of his patience to me. And in particular, I would like to personally thank my Thesis advisor Dr.Ing. Joan García Haro, a second father to me. From the very first day he has managed to guide me through this complex process, giving me important suggestions step by step, as a father would do with his son. Always with a smile in his face, he has never doubted to help me whenever I demanded his attention, regardless of whatever important tasks he might have been carrying out. Thank you very much Joan, just for everything. 


\section{Contents}

$\begin{array}{ll}\text { List of Figures } & \text { xxi }\end{array}$

List of Tables $\quad$ xxvii

I Presentation 1

1 Introduction 3

1.1 Introduction . . . . . . . . . . . . . . . . 3

1.1 .1 Main goals $\ldots \ldots \ldots \ldots \ldots \ldots$

1.1 .2 Justification . . . . . . . . . . . . . . . . 6

1.1 .3 Originality and applicability . . . . . . . . . 7

1.2 Initial objectives . . . . . . . . . . . . . . . . . . 8

1.3 Tools and methodology $\ldots \ldots \ldots \ldots$

1.4 Schedule . . . . . . . . . . . . . . . . . . . . . . 11

1.5 Organization of this Book . . . . . . . . . . . 13

$\begin{array}{lll}2 & \text { State of the art } & 17\end{array}$

2.1 Autonomous driving and VANETs . . . . . . . . . . . . . . 17

2.1 .1 Until the 80 's . . . . . . . . . . . . . . . . 18

2.1 .2 The 80 's . . . . . . . . . . . . . . . . 21

2.1 .3 From the 90 's until now . . . . . . . . . . . . . . . 22

2.2 WAVE IEEE $1609 / 802.11 \mathrm{p}$, standard for wireless communications in VANETs . . . . . . . . . . . . . . . . . . . 26

2.2.1 Access methods and operation modes . . . . . . . . . . 27

2.2.1.1 Experiment 1................... 31

$2.2 .1 .2 \quad$ Experiment $2 \ldots \ldots \ldots \ldots \ldots \ldots$ 
2.2.1.3 Experiment $3 \ldots \ldots \ldots \ldots \ldots$

2.2.1.4 Experiment $4 \ldots \ldots \ldots \ldots \ldots$

2.2.1.5 Experiment $5 \ldots \ldots \ldots \ldots \ldots \ldots \ldots$

$2.2 .2 \quad$ Physical layer . . . . . . . . . . . . . . . . . 33

2.3 Applications of VANETs . . . . . . . . . . . . . . . . . . 35

2.3.1 Active safety applications . . . . . . . . . . . 36

2.3.2 Driving experience enhancement applications . . . . . . . . . . 39

2.3.3 Infotainment applications . . . . . . . . . . . . . . . . 40

2.3.4 Emergency service notifications . . . . . . . . . . . . . . 41

II Cooperative chain Collision Avoidance at high speeds 45

3 Simulation and mathematical validation of a CcCA application for $\begin{array}{ll}\text { VANETs using IEEE 802.11p } & 47\end{array}$

3.1 Introduction . . . . . . . . . . . . . . . . . . . 47

3.2 Related Work . . . . . . . . . . . . . . . . . . . . . . . . . 49

3.3 Simulations and results . . . . . . . . . . . . . . . . 51

3.3 .1 General scenario description . . . . . . . . . . . . . . 51

3.3 .2 Results . . . . . . . . . . . . . . . . . . 53

3.3.2.1 Scenario $1 \ldots \ldots \ldots \ldots \ldots$

$3.3 .2 .2 \quad$ Scenario $2 \ldots \ldots \ldots \ldots \ldots \ldots$

3.3 .2 .3 Scenario $3 \ldots \ldots \ldots \ldots \ldots$

$3.3 .2 .4 \quad$ Scenario $4 \ldots \ldots \ldots \ldots \ldots$

3.4 Stochastic model for $\mathrm{CcCA} \ldots \ldots \ldots$. . . . . . . . . . 60

3.4.1 Characteristics of the model . . . . . . . . . . . . . 60

3.4.2 Computation of the vehicle collision probabilities . . . . . . . 63

3.4 .3 Basic model . . . . . . . . . . . . . . . . . . . . . 66

3.4.3.1 Case 1. Exact computation of collision probabilities with constant kinematic parameters . . . . . . . 66

3.4.3.2 Case 2. Approximate computation of collision probabilities with constant kinematic parameters . . . . . 68

3.4.3.3 Validation and discussion . . . . . . . . . . . . . 69 
3.4.4 Influence of variability on deceleration, velocity and notification delay . . . . . . . . . . . . . . . . . 70

3.4.4.1 Validation and discussion . . . . . . . . . 73

3.4.5 Applications and discussion of the model . . . . . . . . . . 77

3.5 Final remarks . . . . . . . . . . . . . . . . . . . 83

Nomenclature . . . . . . . . . . . . . . . . . . . . . . . 84

4 Testing viability of relay policies for reactive CcCA applications in VANETs

4.1 Introduction and motivation . . . . . . . . . . . . 87

4.2 Related Work . . . . . . . . . . . . . . . . . . . . . 88

4.3 Evaluation and results . . . . . . . . . . . . . . . . . . 90

4.3 .1 General scenario description . . . . . . . . . . . . . . . . 90

4.3.1.1 Algorithm 1 . . . . . . . . . . . . . 94

$4.3 .1 .2 \quad$ Algorithm $2 \ldots \ldots \ldots \ldots$

4.3.1.3 Algorithm 3 ................... 94

4.3 .2 Results . . . . . . . . . . . . . . . . . . 94

4.3.2.1 Simulation case 1. Influence of transmission power . . . 95

4.3.2.2 Simulation case 2. Influence of percentage of $\mathrm{CcCA}$ technology penetration . . . . . . . . . . 98

4.3.2.3 Simulation case 3. Influence of background traffic . . . 100

4.3 .3 Summary of results . . . . . . . . . . . . . . . . . 103

4.4 Final remarks . . . . . . . . . . . . . . . . . . . . . . . . . 104

5 Evaluating communications and IDM in a context of a CcCA appli$\begin{array}{lr}\text { cation for VANETs } & 107\end{array}$

5.1 Introduction . . . . . . . . . . . . . . . . . . . . . . 107

5.2 Related Work . . . . . . . . . . . . . . . . . . . . . . 109

5.3 Communications in IDM . . . . . . . . . . . . . . . . . 112

5.3 .1 General Scenario Description . . . . . . . . . . . . . . 114

5.3 .2 Need for communications . . . . . . . . . . . . . . . 118

5.3.3 Communications in IDM: $T$ conservative approach . . . . . . . . 119

5.3.4 Linear Braking Algorithm (LBA) . . . . . . . . . . . . 120

5.4 Final remarks . . . . . . . . . . . . . . . . . . . . 125 
III Cooperative Collision Avoidance by evasive maneuvering 129

6 Cooperative Collision Avoidance by active evasive maneuvering 131

6.1 Introduction . . . . . . . . . . . . . . . . . . . . . . . . . . . . 131

6.2 Related Work . . . . . . . . . . . . . . . . . . . . . . 133

6.3 Problem statement and formulation . . . . . . . . . . . . 135

6.3.1 State equations and constraints . . . . . . . . . . . 138

6.3.2 Performance measure $\left(J_{L, M}\right) \ldots \ldots \ldots \ldots$

6.3.2.1 $f_{x}$ : minimization of the distance variance . . . . . . 142

6.3.2.2 $f_{v}$ : minimization of the final lateral speed . . . . . . 143

6.3.2.3 $f_{a}$ : minimization of the instantaneous lateral acceleration143

6.3.3 Reformulation as a set of differentiable subproblems . . . . . . . 145

6.3.4 The Modified Gradient Projection (MGP) algorithm . . . . . . . 154

6.3.5 Summary of optimization strategy . . . . . . . . . . . . 155

6.4 Evaluation results . . . . . . . . . . . . . . . . . . . . 155

6.4 .1 Influence of $\theta_{x} \ldots \ldots \ldots \ldots \ldots$

6.4 .2 Influence of $\theta_{v} \ldots \ldots \ldots \ldots \ldots$

6.4 .3 Influence of $\theta_{a} \ldots \ldots \ldots \ldots \ldots$

6.5 Conclusions and future work . . . . . . . . . . . . . . . . . . . . 164

Nomenclature . . . . . . . . . . . . . . . . . . . . . . 166

7 General Conclusions $\quad 169$

7.1 Main summary . . . . . . . . . . . . . . . . . . . . . . . 169

7.2 List of publications . . . . . . . . . . . . . . . . . . . . 172

$7.2 .1 \quad$ Journal Papers . . . . . . . . . . . . . . . . . . . . . . . . . 172

7.2 .2 Conference Proceedings ～. . . . . . . . . . . . . . . . 173

7.2 .3 Book Chapters . . . . . . . . . . . . . . . . . . . . . . 174

7.3 Future worklines . . . . . . . . . . . . . . . . . . . 174

$\begin{array}{ll}\text { A Over the NCTUns Network Simulator } & 177\end{array}$

A.1 Kernel reentering methodology . . . . . . . . . . . . . . . . 178

A.2 Main characteristics of NCTUns . . . . . . . . . . . . . . 179

A.3 CcCA Communications module for NCTUns . . . . . . . . . . . . 181

A.3.1 Mobility module . . . . . . . . . . . . . . . . . . . 183

A.3.2 WSMP Communications' module . . . . . . . . . . . . . . 190 
B Mathematical operations for the stochastic model of Chapter 3

B.1 Computation of the distance traveled by a vehicle for variable velocity and notification delay . . . . . . . . . . . . . . . . 193

B.2 Computation of the distance traveled by a vehicle for variable velocity and deceleration . . . . . . . . . . . . . . . . . . . 199

$\begin{array}{ll}\text { C Mathematical operations on Chapter } 6 & 205\end{array}$

C.1 Derivation of Eq. (6.42) . . . . . . . . . . . . . . . . 205

C.2 Constraints manipulation for the optimization procedure . . . . . . 206

D Trajectory optimization under Gaussian Noise disturbances 213

D.1 Introduction . . . . . . . . . . . . . . . . . . . . . . . . . 213

D.2 Performance criteria and formulation extension . . . . . . . . . . 214

D.2.1 State variability . . . . . . . . . . . . . . . . 215

D.2.2 Measurements variability . . . . . . . . . . . . . 216

D.3 Final remarks . . . . . . . . . . . . . . . . . . . . . . . . . . . . . . . . . . 219

$\begin{array}{lr}\text { References } & 223\end{array}$ 
CONTENTS 


\section{List of Figures}

1.1 Working timeline during the predoctoral stage . . . . . . . . . . 15

2.1 Picture of the proposal for a futuristic car by Norman Bel Geddes in the Futurama's World Fair of 1939 . . . . . . . . . . . . . . 20

2.2 Google Car in action . . . . . . . . . . . . . . . . . . . . . 24

2.3 Intelligent road train in action (SARTRE project) $\ldots \ldots \ldots 25$

2.4 Synthesis of a generic V2X environment operating under IEEE 802.11p 26

2.5 Protocol stack for the WAVE IEEE 1609/802.11p architecture . . . . . . 28

2.6 Graphical representation of the Medium Access methods of the WAVE IEEE $1609 / 802.11$ p standard . . . . . . . . . . . . . . . . 29

2.7 Backoff windows' duration in the EDCA mechanism for IEEE 802.11p . 29

2.8 Bandwidth assigned to each interface in the different Experiments . . . 32

2.9 Spectral use of the physical layer as defined by the DSRC for VANETs . 34

2.10 Frame structure in DSRC for WAVE . . . . . . . . . . . . . . 34

2.11 Critical emergency brake in which communications are used to provide information to anticipate the accident of a leading vehicle . . . . . . 37

2.12 Electronic Toll Collection (ETC) by using IEEE 802.11p V2I (Vehicle to infrastructure) communications . . . . . . . . . . . . . . . 39

2.13 Provision of information about menus in a nearby restaurant: possibility to make a reservation before arrival . . . . . . . . . . . . . . . . 40

2.14 Provision of timely alerts from emergency vehicles of the public service . 41

3.1 Simulation scenario with 802.11 p communication capabilities . . . . . . 48

3.2 Simulation scenario without vehicular communication capabilities . . . . 53 


\section{LIST OF FIGURES}

3.3 Percentage of accidents as a function of the average intervehicular distance $($ Scenario $1, \mathrm{CcCA}$ vs. No-CcCA) . . . . . . . . . . . 54

3.4 Percentage of accidents as a function of the average intervehicular distance (Scenario 2-I, automatic brake vs. human controlled brake, 30 $\mathrm{m} / \mathrm{s}) \ldots \ldots \ldots \ldots \ldots \ldots \ldots \ldots$

3.5 Percentage of accidents as a function of the average intervehicular distance (Scenario 2-II, automatic brake vs. human controlled brake, 40 $\mathrm{m} / \mathrm{s}) \ldots \ldots \ldots \ldots \ldots \ldots \ldots \ldots$

3.6 Percentage of accidents as a function of the average intervehicular distance (Scenario 3, transmission power influence) . . . . . . . . 57

3.7 Percentage of accidents as a function of the average intervehicular distance (Scenario 4, CcCA support influence) . . . . . . . . . . . 59

3.8 The scenario under consideration . . . . . . . . . . . . . . . 61

3.9 Probability tree diagram that defines the model: $S_{i, j}$ represents the state with $i$ collided vehicles and $j$ successfully stopped vehicles . . . . . . . 61

3.10 Probability tree chain for $N=2$ (3 vehicles) and corresponding state transition matrix . . . . . . . . . . . . . . . . 62

3.11 Parameters of the kinematic model used to compute the vehicle collision probabilities . . . . . . . . . . . . . . . . 63

3.12 The distance $l_{i}$ traveled by a vehicle when there is a collision (b) is shorter than the distance needed by it to stop successfully (a), $d_{s, i} \ldots 65$

3.13 Average percentage of accidents versus average intervehicular distance $\bar{s}=\frac{1}{\lambda+L} m$ for basic model, with exact solution (Case 1), approximate solution (Case 2) and Monte-Carlo simulation with a $99.5 \%$ confidence intervals . . . . . . . . . . . . . . . .

3.14 Validation of the model with constant deceleration through the evaluation of three different scenarios. . . . . . . . . . . . . . . . . 74

3.15 Validation of the model with constant notification delay through the evaluation of three different scenarios . . . . . . . . . . . . 75

3.16 Performance of the model with different constant decelerations (a) and message reception delay $(\mathrm{b}) \ldots \ldots \ldots \ldots 78$

3.17 Evaluation of the impact of the parameters' variability on the number of vehicle collisions . . . . . . . . . . . . . . . . . . 79 
3.18 Average percentage of accidents in a low speed scenario with $V_{i} \sim$

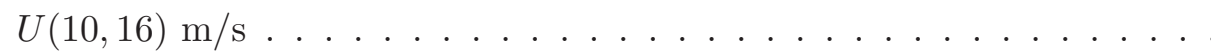

4.1 General scenario for testing relay policies in VANETs with 802.11p communication capabilities . . . . . . . . . . . . . . . . . . . . . 89

4.2 Percentage of accidents as a function of the average intervehicular distance 96

4.3 Merit factor in reception for the system of vehicles . . . . . . . . . 96

4.4 Average accident notification delay . . . . . . . . . . . . . . . . . 98

4.5 Percentage of accidents as a function of the average percentage of technology penetration in the market . . . . . . . . . . . . . . . . . . 99

4.6 Percentage of accidents as a function of the background data traffic delivered by vehicles to the channel . . . . . . . . . . . . . . . . . . . . 101

4.7 Percentage of accidents as a function of the transmission power . . . . 102

5.1 Critical emergency brake in which communications are used to provide information to anticipate the accident of a leading vehicle . . . . . . . 113

5.2 Average percentage of accidents in the platoon (left axis) and evolution of average acceleration variance of vehicles in the platoon (right axis) . . 117

5.3 Minimum obtainable speeds to stop (blue) versus simulation speeds (red) to stop for an average intervehicular distance of 6 meters . . . . . . . . . 117

5.4 Average percentage of accidents within the platoon (left axis) and evolution of average acceleration variance of vehicles in the platoon (right axis $\ldots \ldots \ldots \ldots \ldots \ldots \ldots \ldots$

5.5 Minimum obtainable speeds to stop versus simulation speeds to stop for an average intervehicular distance of 6 meters . . . . . . . . . . . . . 121

5.6 Evolution of average accidents percentage for the four implementation variants studied in this work . . . . . . . . . . . . . . . 126

5.7 Evolution of acceleration variance for the four implementation variants studied in this work . . . . . . . . . . . . . . . . 126

6.1 The general scenario under analysis . . . . . . . . . . . . . . 136

6.2 x-axis projections of elements . . . . . . . . . . . . . 137

6.3 Interpretation of the Inertial Vector on graphs . . . . . . . . . . . . 140 


\section{LIST OF FIGURES}

6.4 Example of $J_{L, M}$ formulation for $L=1, M=1(L=1$ vehicle, $M+2=3$ obstacles ．.. . . . . . . . . . . . . . . . . . 144

6.5 Exemplification of combination reordering for $M=1, L=2$. Each alternative is a weak $(M+1)$-composition of $L \ldots \ldots \ldots$. . . . . 146

6.6 Weak $(M+1)$-composition of $L$ problem, $L$ vehicles into $M+1$ gaps ( $L=1$ vehicle, $M+2=3$ obstacles $) \ldots \ldots \ldots$. . . . . . 147

6.7 Relation of subproblems associated to $J_{L, M}$ for $L=1, M=1(L=1$ vehicle, $M+2=3$ obstacles $) \ldots \ldots \ldots \ldots$. . . . . . . . 149

6.8 Graphic example of how to calculate the $\phi_{m, \beta}(t)$ function $\ldots . . . .151$

6.9 Particular scenario used for evaluation . . . . . . . . . . . 156

6.10 Evolution of trajectories for $M=1$ ( $M+2=3$ obstacles), $L=2$ vehicles and $\theta_{x}=1, \theta_{v}=0, \theta_{a}=0, t_{f} \in\{0.9,1,1.1\} \mathrm{s} \ldots \ldots \ldots 157$

6.11 Speeds for $\mathrm{M}=1, \mathrm{~L}=2$ and $\theta=[1,0,0], t_{f} \in\{0.9,1,1.1\} \mathrm{s} \ldots \ldots 158$

6.12 Accelerations for $\mathrm{M}=1, \mathrm{~L}=2$ and $\theta=[1,0,0], t_{f} \in\{0.9,1,1.1\} \mathrm{s} \ldots 159$

6.13 Evolution of trajectories for $M=1$ ( $M+2=3$ obstacles), $L=2$ vehicles and $\frac{\theta_{v}}{\theta_{x}}=\{100,10,1,0.1\}, \theta_{a}=0, t_{f} \in\{0.9,1,1.1\} \mathrm{s} \ldots \ldots \ldots$

6.14 Mobility profile of vehicle $1, \frac{\theta_{v}}{\theta_{x}}=\{100,10,1,0.1\}, \theta_{a}=0, t_{f}=1 \mathrm{~s} \ldots 161$

6.15 Mobility profile of vehicle $2, \frac{\theta_{v}}{\theta_{x}}=\{100,10,1,0.1\}, \theta_{a}=0, t_{f}=1 \mathrm{~s} \ldots 162$

6.16 Evolution of trajectories for $M=1$ ( $M+2=3$ obstacles), $L=2$ vehicles and $\frac{\theta_{v}}{\theta_{x}}=\{10,1,0.5\}, \frac{\theta_{a}}{\theta_{x}}=\{0,0.1,0.5\}, \theta_{x}=1, t_{f}=1.2 \mathrm{~s} \ldots \ldots 3$

6.17 Speed profiles of vehicle 1 , for $\mathrm{M}=1, \mathrm{~L}=2$ and $\frac{\theta_{v}}{\theta_{x}}=\{10,1,0.5\}, \frac{\theta_{a}}{\theta_{x}}=$ $\{0,0.1,0.5\}, \theta_{x}=1, t_{f}=1.2 \mathrm{~s} \ldots \ldots \ldots \ldots \ldots \ldots \ldots \ldots \ldots \ldots \ldots \ldots$

6.18 Acceleration profiles of vehicle 1 , for $\mathrm{M}=1, \mathrm{~L}=2$ and $\frac{\theta_{v}}{\theta_{x}}=\{10,1,0.5\}$, $\frac{\theta_{a}}{\theta_{x}}=\{0,0.1,0.5\}, \theta_{x}=1, t_{f}=1.2 \mathrm{~s} \ldots \ldots \ldots \ldots \ldots \ldots \ldots \ldots \ldots \ldots \ldots$

A.1 Kernel reentering methodology of NCTUns . . . . . . . . . . . . . 179

A.2 Communications' module flowchart, part 1 . . . . . . . . . . . 184

A.3 Communications' module flowchart, part 2 . . . . . . . . . 185

A.4 System's state diagram . . . . . . . . . . . . . . . . . . 186

A.5 Sampling period graph for calculating speed during braking . . . . . . . 190

C.1 Particularization cases to obtain the partial derivative $\frac{\partial \phi_{m, \beta}(k)}{\partial a_{p}(s)} \ldots 206$

D.1 Scenario considered for the evaluation of Gaussian noise on trajectories 215 
D.2 Kalman Filter effect on trajectories for $J_{D 1}$ and $J_{D 2}$ under measurement noise $\left(\sigma_{z} \in\{0.1,1,5\}\right) \ldots \ldots \ldots \ldots \ldots \ldots \ldots$

D.3 Degree-2 polynomials for regression of MSE under measurement noise $\left(\sigma_{z} \in\{0.1,1,5\}\right)$, for $J_{D 1} \ldots \ldots \ldots \ldots \ldots \ldots \ldots \ldots$

D.4 Percentage of distance respect to the lateral optimum position under measurement noise $\left(\sigma_{z} \in\{0.1,1,5\}\right)$, for $J_{D 1} \ldots \ldots \ldots \ldots$

D.5 Degree-2 polynomials for regression of MSE under measurement noise

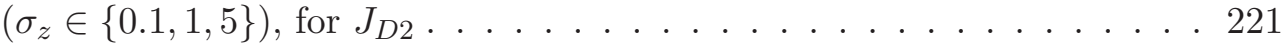

D.6 Percentage of distance respect to the lateral optimum position under measurement noise $\left(\sigma_{z} \in\{0.1,1,5\}\right)$, for $J_{D 2} \ldots \ldots \ldots \ldots 221$ 


\section{List of Tables}

2.1 Comparison between simulation and real experiments . . . . . . . . 23

2.2 Configuration values for electronic testbench . . . . . . . . . . . . 30

2.3 General classification of applications in VANETs according to the four proposed categories . . . . . . . . . . . . . . . 43

3.1 Configuration parameters for the simulation scenarios . . . . . . . 52

3.2 Merit factor comparison for Scenario 1 . . . . . . . . . . . . 55

3.3 Merit factor comparison for Scenario $2(30 \mathrm{~m} / \mathrm{s}) \ldots \ldots . \ldots 56$

3.4 Merit factor comparison for Scenario $2(40 \mathrm{~m} / \mathrm{s}) \ldots \ldots . . \ldots 57$

3.5 Merit factor comparison for Scenario $3 \ldots \ldots$. . . . . . . 58

3.6 Merit factor comparison for Scenario 4 . . . . . . . . . . . . . 60

4.1 Configuration parameters for the simulation cases . . . . . . . . . . . 95

4.2 Successful Delivery and Packet Collisions Ratio (SDR, PCR), tx. power

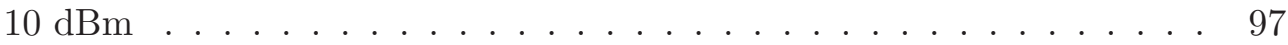

4.3 Successful Delivery and Packet Collisions Ratio (SDR, PCR), tx. power $28 \mathrm{dBm} \ldots \ldots \ldots \ldots \ldots \ldots \ldots \ldots$

4.4 Average accidents percentage for general background data traffic . . . . 100

4.5 Average accidents percentage for all Transmission Power Values . . . . . 102

5.1 Configuration parameters for all simulations . . . . . . . . . . 116

5.2 Characterization of transmission packets . . . . . . . . . . . . . 119

5.3 Specific simulation parameters for the $T$ conservative approach . . . . 120

6.1 Configuration parameters for the evaluation cases . . . . . . . . . 156

D.1 Configuration parameters for the scenario in Appendix D . . . . . . 215 


\section{Part I}

\section{Presentation}





\section{1}

\section{Introduction}

\subsection{Introduction}

Human beings have always needed to move. From walking to flying, the history of human transportation has always comprised a lot of different technologies, methods, timing requirements and safety issues. Modes of transport can be divided into aviation (planes, helicopters...), ship transport (ships, sailboats...) and ground vehicles, including rail (trains) and road transport (cars, motorcycles...). In particular, road transport, has grown to be the most prominent mode of transport in the world, specially during the last 50 years, due mainly to the massive commercialization of ground vehicles in rich and emerging economies $(1)^{1}$. Either for civil transport or for commercial purposes, ground vehicles have become the most relevant support for the growth and sustainability of developed societies, as well as an impulse for emerging countries (2). Actually, the huge network of vehicles currently operating exists thanks to the research taken during the past decades on all aspects of car driving: mechanical elements of the car, electronic devices, safety measures, fuels and so on. Specifically, safety is crucial due to the negative implications on passengers that a lack of it can entail. Seat belts, anti-blocking systems (ABS), airbargs, among others, are technologies designed by car manufacturers in order to increase passengers' safety and improve the driving experience.

However, despite all these new safety technologies and due to the sometimes unpredictable behavior of humans while driving, car accidents involve high costs to the

\footnotetext{
${ }^{1}$ Citation formatting will consist of red numbering delimited by square brackets in the rest of the Book.
} 


\section{INTRODUCTION}

healthcare systems of rich and emerging countries (for example, in the USA, where costs reached $\$ 100$ billion in 2010 (3)). For this reason, new emerging technologies are receiving most of the attention in the global research community so as to build advanced vehicles which can reduce these issues to a minimum. In particular, wirelessly interconnected autonomous vehicles are foreseen to be the next giant step in the automotive industry, providing ground transports with advanced intelligence and sustainability (4). Numerous research groups and industrial manufacturers are making a great effort to introduce robotics and wireless communications' support into the next generation vehicles for an improved safety on the road and to offer an attractive service of telematic apps which will make car journeys much more comfortable for passengers.

In order for autonomous vehicles ${ }^{1}$ to be a reality, numerous practical and ethical concerns must be solved at first. In practice, such automobiles will rely on advanced smart processing systems that will be carried on board or installed in processing servers far away. These systems will need information collected by sensors on board and/or by sensors of other vehicles or entities. Thus it will also be necessary to count on wireless technologies allowing the transmission of information vehicle to vehicle (V2V) or vehicle to infrastructure (V2I), so as to provide servers with the necessary data to process. Since vehicles operate under very different environmental conditions, communications are a very complex subject when dealing with information exchange between vehicles and/or other entities. Actually, due to the great complexity of this sort of networks, many initiatives have been undertaken worldwide to study the standardization of general ITS procedures for general inter-operability between brands and markets. Ranging from technical aspects regarding protocol specifications to general application requirements, many consortiums have been financed (either privately or publicly) to evaluate the feasibility of such a big network and the problems associated to the different penetration stages in the market. Due to the importance of inter-operability, these initiatives acquire a central responsibility in the future success of this still emerging technology. Some important examples like the Prometheus Project, which initiated a paneuropean collaboration scheme between different universities and organisms to promote research on autonomous vehicles (5), and, more recently, the DARPA Grand Challenge, for driverless car guidance (6). In this Book we will not provide detailed information

\footnotetext{
${ }^{1}$ In the rest of the Book we will refer to wirelessly interconnected autonomous vehicles simply as autonomous vehicles.
} 
about the different consortiums and initiatives in this regard that have appeared so far (for detailed information on them, please refer to the introductory part in the Thesis led by Alonso in (7)). However, we will use the next chapter to provide a general state-of-the-art description of the most important technologies related mainly to both autonomous vehicle guiding, and intervehicle communications, mentioning those initiatives and consortiums that might be more closely related to our main proposal. We will complete the chapter with further information about the standard for wireless communications in vehicular environments, in particular, the family of standards IEEE $1609 / 802.11$ p (8), designed by the IEEE organization to be the scalable framework of operation for general wireless communications in vehicular environments. Finally, in the last subsection of this chapter we will present the most important applications that will be available in vehicles dealing with such technologies as well as the most relevant benefits of using such services. This last subsection will serve us to introduce the central topic which has become the main research point of this Thesis: Cooperative Collision Avoidance at high speeds.

\subsubsection{Main goals}

The main objective of this Thesis is the proposal and evaluation of Cooperative Collision Avoidance applications for high-speed vehicles under critical risky circumstances. In particular, for situations in which vehicles might need to execute timely maneuvers in order to avoid crashing with other neighbors. In this regard, we have divided the predoctoral stage into two main phases:

1. Cooperative chain Collision Avoidance (CcCA). In the first part of this Thesis we have considered the analysis, either by simulation as well as by mathematical methods of single-lane chains of vehicles that have to react to a sudden stop of a vehicle (or the presence of an object) in the front part of the chain, by sending Collision Warning Messages (CWM) in order to reduce the probability of accident (or at least mitigate its impact in case it is unavoidable), and braking to avoid the crash (assuming that evasive maneuvers are not possible, i.e. worst case situations).

2. Cooperative Collision Avoidance by evasive maneuvering. In the second stage of the Thesis we have centered our attention in the study of optimum evasive 


\section{INTRODUCTION}

trajectories for vehicles on a cooperative scheme that allows them to benefit from lateral gaps on the road to avoid collisions with suddenly appearing (undetected) objects on the road.

\subsubsection{Justification}

We know from the present state of the art in Chapter 2 that currently under-development autonomous selfguiding vehicles rely on sensors to make real-time probabilistic-based decisions in order to follow a certain prefixed route, generally under a mid-low level of risk, such as urban environments, heavy traffic, etc. (see, for example, the work led by Sebastian Thrun, from the University of Standford in the USA, on autonomous vehicles like the media Google Car (9)). Although some cases like the Google Car (10) have already crossed the boundary of 300,000 miles without accidents of any type on the american roads, there still remains the open issue of investigating how autonomous vehicles can handle unpredictable circumstances, mainly those ones where safety might be notably reduced (heavy rain, icy asphalt, suddenly appearing objects on the road...) (11). Actually, this becomes confirmed by the, for instance, Google Inc. global job offer where technicians are demanded in order to propose and evaluate reactive policies for autonomous vehicles under such circumstances (12).

Additionally, the use of communications to complement robotic-assisted vehicular technology can be highly beneficial, since in practically all cases vehicles will obtain not only information registered due to the on board sensors, but also the traffic state of nearby vehicles, their short-term motion trajectories, more detailed environmental conditions, and so on. With an adequate processing scheme, vehicles can even anticipate the state of the nearby vehicles and phenomena even earlier than with autonomous vehicles working isolated.

Therefore, we propose to use communications (with their functionality provided by the IEEE 1609/802.11p family of standards) to improve safety of driverless vehicles, specially under unforeseen circumstances which might entail a remarkable risk to the passengers. In particular, for Cooperative Collision Avoidance in situations where vehicles might incur in a multiple chain collision on high-speed roads, as well as in cases which vehicles would have to execute timely evasive maneuvers to evade the collision with unpredicted objects appearing on their way. 


\subsubsection{Originality and applicability}

Autonomous self-guiding vehicles are already capable of operating properly in structured road networks (see (13)). Under, in general, controlled circumstances, noncommercial self-driving vehicles can cover currently distances up to 300,000 miles and even more without accidents of any kind. However, the next natural step is to provide them with communications support, so that even more complex strategies can be followed in unpredictable situations so that cooperativeness can help vehicles behave in a much safer manner, something that in isolation might not be possible.

In particular, despite research on safety applications for VANETs has already been tackled to some extent, very little has been conducted for both CcCA and CCA so far. Actually, works on Cooperative chain Collision Avoidance have focused primarily on the design and evaluation by simulations of pure communication protocols, paying exclusive attention to its performance in terms of the end-to-end delay, throughput, etc. (like the works of (14) and (15)) without evaluating extensively the impact on the number of accidents. On the other hand, regarding cooperative evasive maneuvering, most works have concentrated on optimizing trajectories in regular traffic/environment situations for individual self-guided entities (aerial, land and submarine vehicles), without focusing in depth on the characteristics of mobility for autonomous vehicles under unexpected situations, normally under harsh constraints of time. We will leave the details on the Related Work about the two approaches for the corresponding forthcoming chapters, so as to better introduce the information presented in the specific context. It is worth saying, whatsoever, that our work has been originally conceived taking as reference the studies led by Hartenstein (see his book on VANETs (16)), the study on communication protocols for relaying in vehicle platoons (by Biswas S. in (17)), the car-following models IDM (Intelligent Driver Model) and HDM (Human Driver Model), in (18), and all the mathematical theory of Optimal Control, in the outstanding book of Donald E. Kirk, in (19).

As we will see, the proposals appearing in this Thesis are aimed at operating in situations in which vehicles have to execute harsh maneuvers within very small intervals of time. Despite the extension of the Thesis, investigating on safety applications with timing constraints is complex, but in reality it will be very useful and, more importantly, necessary for future autonomous interconnected vehicles to operate reliably even under 


\section{INTRODUCTION}

unexpected circumstances. Most of the work on regular driving is already done, but now it is the turn to improve reliability of vehicles in unpredictable situations, for example, (12).

\section{$1.2 \quad$ Initial objectives}

In this Thesis we plan to investigate two specific situations of potential risk of accident in road traffic: i) vehicles in danger of chain collisions in platoons and how communications can be used to send warning notifications at an earlier stage to alert them about the event ( $\mathrm{CcCA}$ ), and ii) vehicles in danger of colliding against obstacles at short distances and high speeds, and how cooperative communication schemes can be used to execute timely maneuvers in order to avoid crashing (evasive CCA).

Regarding CcCA, we plan to extend the functionality of our main networking simulation tool NCTUns 6.0 (see Appendix A) by implementing inter-module in-car communications, between the mobility manager (which handles the movement of the vehicle) and the communications' module (WSMP module). This allows the car to react in terms of a modified motion pattern after the reception of some information (either CWMs or beacons) by the communications' module, and viceversa. The mobility manager has been adapted to implement vehicular motion as recreated by cars belonging to high-speed platoons. We intend to use this implementation to analyze the problem of anticipating an accident to forthcoming vehicles in a platoon of vehicles by using communications under three main premises:

- Simulation of a CcCA application for different platoon lengths, under different technology penetration ratios, without relay (retransmission of self-produced packets without intermediate delivery), for different values of the transmission power and comparing human assisted driving to autonomous driving. Validate results by means of a stochastic model which describes mathematically the characteristics of sudden braking events in worst-case situations of platoon collisions (cars can only brake without taking evasive maneuvers).

- Simulation of a CcCA application under the same conditions of the first point, this time applying different relay algorithms to show under which circumstances these schemes can be useful or even necessary to improve safety of the cars in 
hard braking events. Study of the number of percentage of accidents in the platoon for scenarios with different transmission power values, background noise, different technology penetration ratios, and with the evaluation of useful performance metrics (SDR, Succesful Delivery Ratio; PDR, Packet Delivery Ratio; Utility Factor).

- Implementation of a realistic car-following model for vehicular traffic in the mobility manager of NCTUns, to compare more realistically the benefits of using communications in hard braking events. Design of policies for improving driving comfort even in situations of emergency braking, in particular, with the LBA (Linear Braking Algorithm).

In CCA for evasive maneuvering, we plan to develop a mathematical optimization method in order to solve the problem of sudden trajectory tracing for cases in which vehicles need to cooperatively execute very rapid maneuvers in order to avoid one or more obstacles appearing ahead. In this case, the main objectives are classified next:

- Formulation of the problem for one vehicle to reach an optimum position in minimum time as regards the maximization of the lateral distance with respect to the lateral crash barriers. Inclusion of more performance measures to study the flexibility in the trajectory tracing procedure.

- Extension of the mathematical model for a scenario with an arbitrary number of vehicles and obstacles. Vehicles need to agree what the best cooperative strategy might be to avoid crashing against one or more obstacles appearing ahead, and profiting from the remaining free gaps appearing on the laterals of the obstacles in order to continue their trajectories along these free spaces. Performance metrics regard maximization of the lateral distance, minimization of the lateral speed at the end of the trajectory, and minimization of the total acceleration along the path.

- Evaluation of trajectories affected by random gaussian noise, and usage of Kalman Filtering to reduce the impact of path deviations from the optimum path. 


\section{INTRODUCTION}

In all cases, scenarios contain a finite number of vehicles, since the subject of research deals with practical situations of the vehicular traffic. Although not contemplated in this work, this allows for the experimentation with real autonomous interconnected vehicles, at a not so high costs (which might be necessary in cases where communication protocols might be tested for large vehicular networks at bigger scales).

\subsection{Tools and methodology}

All experiments in this Thesis have been led either by simulation and mathematical evaluation. For simulation we chose the NCTUns 6.0/Estinet network simulator. This election was made because by the time we had to decide which tool to use, NCTUns had already implemented the WAVE IEEE 1609/802.11p standard family. Furthermore, due to the Re-entering methodology offered by NCTUns, we could simulate vehicles with real pseudo-interfaces that could help us get more reliable results. In simulation scripting, we made use of the BASH and Python shell scripts in order to set up all network configuration files as well as the results formatting files.

For the mathematical evaluations we finally chose to work with Matlab, mainly because of the already experience of the Author with the environment, and the great support that this environment has in the scientific community for advanced mathematical evaluations.

Regarding results formatting, we relied on GNUplot and the Matlab plot function for graph illustrations. For general figures and pictures, we used the xFIG plotting function. The AWK text processing tool was also very useful, specially during the first stage of the predoctoral phase. Articles were written either using the Latex environment or the Office Word 2010 program.

The general methodology of this Thesis is based on an earlier study of the State of the Art of general applications for VANETs, as well as an in-depth characterization of the standard for vehicular communications WAVE (see references (20) and (21), in Spanish, where an exhaustive work on this two fields was carried out). Keeping in mind the original criteria of investigating how communications can be used to improve safety under critical risk-of-accident events, we went on with a more application-specific state-of-the-art research where we sought the last tendencies in both CcCA and CCA for evasive maneuvering, either by simulation as well as by mathematical modeling. 
After the main platforms to work (NCTUns and Matlab) were chosen, we developed the main implementations regarding the proposed applications:

1. Cooperative chain Collision Avoidance (CcCA), for which we first carried out a variety of simulations to evaluate the behavior of vehicular platoons under critical emergency braking when communications are used to anticipate information about collisions on the front part of the chains. Then, a mathematical model for this type of scenarios was led to validate results obtained by simulations. In the following stage we extended the capabilities of our simulator by including relaying algorithms in order to increase the coverage area and analyze the performance of the proposed protocols. After that we implemented the IDM/HDM car following models in order to evaluate the performance of our proposals as compared with realistic human-assisted car-traffic in the same situations.

2. Cooperative Collision Avoidance for evasive maneuvering, where we change the main focus and tackled the problem as the calculation of evasive trajectories for vehicles having to avoid the collision with ahead obstacles by using lateral free gaps in order to reorientate their paths. Although the next following step is to evaluate the performance of the optimization scheme for a network of vehicles in a simulation platform (NCTUns is a good option for this case), this is out of the scope of this Book, and consequently left as future work.

\subsection{Schedule}

As can be inferred from the previous sections, we differentiated the predoctoral stage into two main phases. We have illustrated a graphical timeline (represented in Fig. 1.1) to give the reader at a glance a general vision about the different working periods comprising the Thesis.

In the first phase, from the beginning (September 2009) until February 2010 we started by implementing our CcCA application in NCTUns, simulating different scenarios and testing its functionality with relevant performance metrics. Its resulting paper (22) was accepted and presented in the International Conference on Communications (ICC) in the 2010 Edition, held in Cape Town (South Africa). At the same time we started including relaying in the implementations of our simulator, we began 


\section{INTRODUCTION}

to model our CcCA application with a stochastic approach for the purpose of validation. The study on relaying comprised three months (until June 2010), turning into a paper presented in the Wimob 2010 Conference, (23), held in Niagara Falls, Ontario (Canada), in 2010. On the other hand, the stochastic model needed almost one year in order to be fully completed (with the final version being accepted by the T-ITS on July 2011, see (24)). During the first half of 2011 we devoted our efforts to the simulation of our chosen car-following models IDM/HDM in NCTUns, with a final submission to the RSS ( $3^{\text {rd }}$ International Conference on Road Safety and Simulation) conference, which resulted in an eventual acceptance of the article, see (25).

The second stage of the Thesis started in September 2011, and coincided in time with a research stay of the Author in the PEL (Performance Engineering Laboratory) of the UCD (University College Dublin) in the Republic of Ireland (which lasted six months, from October 2011 until March 2012). Until the end of 2011, the Author devoted his efforts to investigating CCA for evasive maneuvering by first carrying out a detailed search on related work to afford the design of the optimization model. From March 2012 until November 2012, the Author focused on the statement and characterization of the model, with final results in the paper accepted at the Wimob Conference of 2012 (held in Barcelona), see (26), and the article in Magazine Future Internet, (27), and a manuscript submitted to the Journal of Transactions in Intelligent Transportation Systems, (28), the last two still being under the review process by the time of writing this document.

Although out of the scope of the contents of this Book, the Author plans to continue with research on CCA for evasive maneuvering at least until the end of the FPU fellowship that has financed his work during the predoctoral period, by:

- Analyzing the behavior of the model for a large variety of scenarios and optimize its functionality according to the premises of minimum risk of accident, and maximum driving comfort.

- Evaluating the model in a network simulator in order to test the functionality of different communication protocols implementing the main characteristics of our proposal. 


\subsection{Organization of this Book}

This Book is divided into three main differentiated parts. In Part I, we have used the present Chapter to introduce the main objectives and evaluation methodologies of this Thesis. Continuing with Chapter 2 of Part I, we highlight the milestones of autonomous interconnected vehicles until the present day, devoting two Sections for the description of the main characteristics featuring the standard WAVE, and the types of possible applications that will appear in VANETs, respectively.

Part II will focus on our proposal on CcCA. It will be divided into three chapters. In particular, Chapter 3 will present, on the one hand, the simulation results after using NCTUns to evaluate the performance of the proposed application for chains of vehicles operating in different scenarios. On the other hand, a specific stochastic mathematical model will be presented to validate simulations and show some specific results when using this mathematical method under different configurations of the main scenario. Chapter 4 will analyze three specific algorithms for information relaying in CcCA scenarios, with a detailed performance evaluation of the main metrics in order to characterize the communications' channel. We will finalize Part II with Chapter 5 , where we will compare the performance of our communications' scheme with carfollowing models like IDM and HDM, and propose an algorithm for a more comfortable braking process (LBA) in cases where it is possible.

Part III will contain Chapter 6, where we will present an exhaustive optimization analysis of the typical problem of Collision Avoidance by evasive maneuvering, but using a cooperative decision-making approach to obtain the best set of solutions to this problem. In Part III, Chapter 7, we will eventually include the main Conclusions of the Thesis, along with the complete list of publications (journal papers, conference proceedings and book chapters) authored and coauthored by the Doctorand during the predoctoral stage.

After the General Conclusions, we will present four appendices regarding some additional aspects about the material presented so far. Appendix A will introduce the main characteristics of our networking simulation platform, NCTUns 6.0, particularly, applied to the specific approach (CcCA) we deal with in this Thesis. Appendix B will describe some necessary mathematical operations to obtain the mathematical results of the stochastic model for CcCA of Chapter 3. On the other hand, Appendix C 


\section{INTRODUCTION}

will present some additional operations to explain the mathematical derivations of the optimization model in Chapter 6. Lastly, Appendix D will give a brief study on how optimum trajectories (as calculated by the optimization procedure of Chapter 6) can be affected by Gaussian noise processes, and which solution can be given to this phenomena to reduce its impact. 


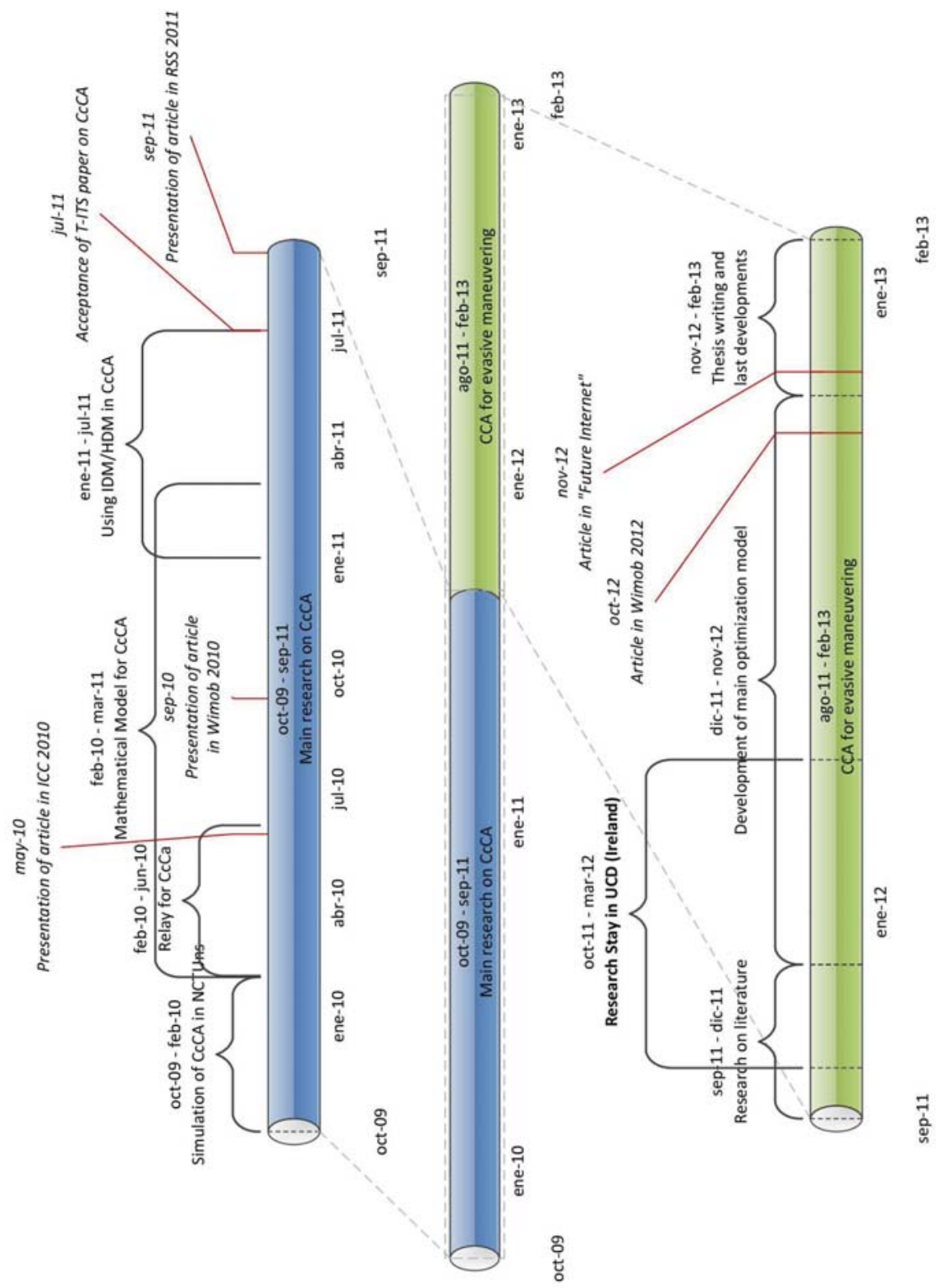

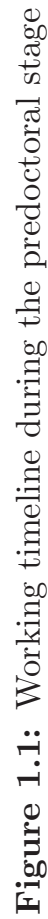




\section{2}

\section{State of the art}

Due to the enormous quantity of information that is already available in the literature to know the history of vehicular traffic technology, in this chapter we present a general State of the Art in terms of the, according to the Authors' point of view, most important landmarks in research on autonomous car guiding and vehicular connectivity. We devote Section 2.1 to a brief presentation of the most relevant inventions on related car technologies that the Industry and the Academia have made so far. Section 2.2 aims at introducing the general implementation concepts that build the Protocol Family of Standards for Wireless Access in Vehicular Environments WAVE IEEE $1609 / 802.11 \mathrm{p}$ (8). This standard is designed to regulate communications between interconnected vehicles and future roadway stations for providing advanced user services in the car. An exhaustive presentation of these referred applications is left to Section 2.3, where we highlight the most important characteristics of future applications operating in VANETs. For more detailed information on the concepts presented, please read the reference book written by researchers of the Carnegie Mellon University in USA on robotic car guidance Intelligent unmanned ground vehicles: autonomous navigation research at Carnegie Mellon (29), or the more recent work by Hannes Hartenstein from University Karlsruhe VANET Vehicular Applications and Inter-Networking Technologies (16).

\subsection{Autonomous driving and VANETs}

It is rather easy to perceive from general research on ITS in the past years that there have been two clear tendencies to afford the investigation in these matters. At first 
hand, we can stand out the investigation on all aspects regarding the implementation of driverless capabilities for automatic guidance in ground vehicles. The second tendency is, however, to pay more attention to the evaluation of vehicular communication protocols, in particular, to allow the exchange of information between pairs of vehicles and between vehicles and infrastructure for general (not necessarily safety) applications. In this regard, some important research organizations like the SwRI (Southwest Research Institute), in San Antonio, Texas (USA), agree that the next natural stage is to integrate both technologies (as long as their respective implementation maturity is reasonable), and evolve from a vehicular communication-enabled automation support, to a fully cooperative, autonomous operation where vehicles build a complete self-contained network of cooperative nodes that dynamically exchange information for the welfare of the whole system (30). Another example of this convergence can be also noticed in the research led by the Spanish CSIC (in Spanish: Consejo Superior de Investigaciones Científicas, Scientific Investigations' Main Counsel), as we will see in a later section.

Therefore, it is interesting to analyze qualitatively, at least as a starting point, up to which degree both approaches have converged so far (in terms of mutual complementarity), since in the future these two technologies will necessarily have to coexist and simultaneously benefit from each other, specially for those safety-related user services. This section presents the most important milestones arising during the past decades, in terms of both autonomous car driving and vehicular communications' support, focusing eventually on the most current technologies already on operation, or under development, and trying to extract the most important common aspects, specially as regards safety-related applications.

\subsubsection{Until the 80's}

As many surveys on autonomous robotic motion (like the work in (16)) agree, concepts on both robotics for self-guided vehicles as well as communication systems for active vehicle-to-vehicle connectivity appeared for the first time in the World Fair of 1939, exhibited by the Norman Bel Gedde's Futurama prototype car (see Fig. 2.1), envisioning many of the technological improvements that Intelligent Transportation Systems would hold in the following 20 years. Twenty years later, and influenced by the advances in mobility in the United States, the Mechanical Engineering Laboratory and the National Institute for Advanced Industrial Science and Technology in Japan 
developed an Automated Highway System (AHS) to control the lateral motion of a vehicle by magnetic attraction (thanks to the installation of an inductive cable under the road surface and using a couple of on-board bumpers to govern the car dynamics (31)), and reaching peak speeds of almost $100 \mathrm{~km} / \mathrm{h}$. Due to this new invention, it was possible for the first time in history to autonomously govern the driving direction of a vehicle without human intervention. However, using this sort of guidance rails for car mobility made the motion task impractical, thus needing new methods to allow vehicles to better react to the environment, while respecting their steering and braking capabilities. Therefore it can be considered that the first realistic approach in autonomous car research took place at the end of the seventies, with the great effort carried out by the Tsukuba Mechanical Engineering Lab team led by S. Tsugawa et al. (32) evaluating the mobility of a self-driving car on a pre-established road, reaching a peak speed of 30 $\mathrm{km} / \mathrm{h}$. This vehicle implemented a stereo visual-processing system which could analyze graphical information covering a maximum range of 40 degrees and up to a distance boundary of $20 \mathrm{~m}$. Despite using hardwired technology (by that time computers were not fully available yet), this vehicle could detect lateral crash barriers and central lane marks, and simultaneously trace a low-speed trajectory which guided the vehicle along the road. The same team designed an advanced version of the vehicle by equipping it with dead-reckoning ${ }^{1}$ capabilities for exact position detection. Although it drove up to a speed of $10 \mathrm{~km} / \mathrm{h}$, this new edition of the car can be considered to be the first one to use visual aids for obstacle avoidance purposes.

On the other hand, first investigations on wireless vehicular connectivity did not arrive until 1970, when the work of Rosen et al. (33) appeared, becoming one of the milestones in vehicular communications' research and the precursor of the current GPS (Gobal Position Systems). In the referred work, the authors proposed a routing assistance system for the drivers by installing roadside units in critical locations. These roadside receivers would be ready to reply to an on-board unit at the time of approaching an intersection in order to inform the driver about the optimal path to choose, according to a prestablished destination configured in advance by the user. Working at a frequency rate of $170 \mathrm{KHz}$, loop antennas were installed in the rear part of the vehicles and the roadside units would be equipped with the same type of antennas. By

\footnotetext{
${ }^{1}$ Dead reckoning is a simple mathematical procedure using trigonometric expressions to infer the current location of a robot.
} 


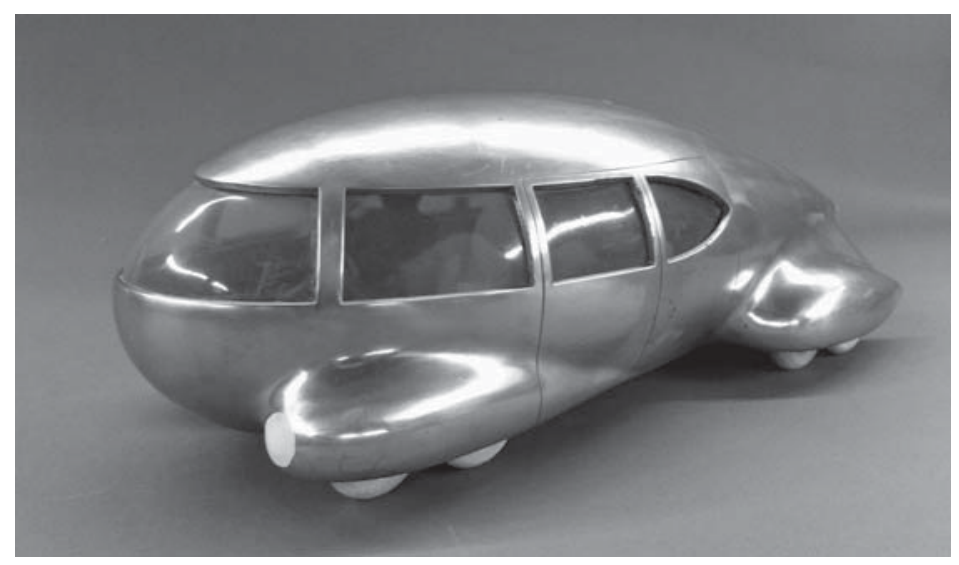

Figure 2.1: Picture of the proposal for a futuristic car by Norman Bel Geddes in the Futurama's World Fair of 1939

the end of the eighties, this system proved not to be profitable due to the high costs of the road infrastructure (16). Independently from autonomous car research, in Japan the Agency of Industrial Science and Technology, led by the Ministry of International Trade and Industry (MITI), designed and carried out during the seventies a framework to regulate car traffic in Japan and improve safety and efficiency while driving: the CACS (Comprehensive Automobile Traffic Control System) also evaluated an early vehicular ad-hoc network by proving Vehicle-to-X (V2X) communications at datarates of about 48 kbps. As described exactly by Kawashima (34) (and introduced in (16)), the objectives of the project, still valid nowadays, are summarized next:

- Reduction of road congestion

- Reduction of exhaust fumes caused by traffic congestion

- Prevention of traffic accidents

- Enhancement of public and social role of automobiles

In technical terms, these four objectives were reformulated as follows (34):

- To guide drivers along most appropriate routes in order to avoid congestion or prevent air pollution

- To provide useful information in order to assist safe driving 
- To give priority of transit to public or emergency vehicles

- To provide information promptly to drivers in case of emergency

Among the numerous consortiums and initiatives created by different government agencies and public administrations for the progress of car traffic technology, the project CACS can be considered the first real initiative led by a public administration to promote the evolution of car driving as a whole.

It is possible to conclude here that Japan played a big role in the initialization of research on vehicular communications and autonomous car driving initiatives. We will see in the next sections that this heavily influenced the fascinating technologies yet to come.

\subsubsection{The 80's}

In 1980 Ernst Dickmanns and his collaborators from the Bundeswehr University Munich in Germany designed a visually-aided robotic van which could drive autonomously on roads without traffic reaching speeds of $100 \mathrm{~km} / \mathrm{h}$ (35). Subsequently, the European Commission created the Eureka Prometheus Project (PROgraMme for an European Traffic of Highest Efficiency and Unprecedented Safety, 1987-1995), the largest initiative on advanced vehicle technology research ever, for which around $\$ 1$ billion were assigned. The Prometheus project stimulated the progress in the research of vehicular communications by organizing the objectives into three subprograms (16), namely:

1. PRO-CAR: driver assistance by electronic systems.

2. PRO-NET: vehicle-to-vehicle communications.

3. PRO-ROAD: vehicle-to-environment communications.

The main action points of the Prometheus project were to increase European traffic safety, reduce air polluted emissions, enhance traffic efficiency and open new economic perspectives by using new communication technologies both in vehicle to vehicle as well as vehicle to roadside connectivity (5). Considering whatsoever that the system would be evolutionary, the most relevant changes would come from the application of optimum route guidance and road throughput, as predicted by (36). Yet, the years to come would prove that autonomous car driving without human intervention would 
receive major attention in the associated research circles, as we will see. From the technical point of view it is worth mentioning the work of Dabous and Huitema (37), with an early study of the communication requirements that an ad-hoc communications protocol operating in vehicle-to-vehicle connectivity would need.

Almost simultaneously in the USA, the DARPA-funded Autonomous Land Vehicle (ALV) could drive autonomously on typical roads by using laser radar, computer vision and autonomous robotic control (38) for the first time in history. This invention became actually the origin of the current technologies that some companies like Google Inc. use in order to perform self-guided driving with their cars, therefore its importance (39). An advanced version of the ALV was tested on a road-off map by the HRL Laboratories, showing the capabilities of autonomous driving on even complex surfaces with slopes, vegetables and rocks (40).

We notice that whereas approaches on communication protocols for VANETs were on their initial stage (at least, most related research was first focused on the theoretical part of the general intervehicular communication analyses), autonomous car driving paid attention exclusively to the experimentation with self-guiding vehicular motion, but under very specific environmental conditions. However, it was not until the late nineties that autonomous driving suffered a drastic change in the basic paradigm: the use of probabilistic robotics (41), supported by the early work on linear filtering estimation originally conducted by Kalman in 1963 (42).

\subsubsection{From the 90's until now}

It is during the last twenty years that the field of VANETs and autonomous car driving have both attracted the highest attention of the research community. It can be considered that most of the research carried out on safety applications for interconnected autonomous vehicles has been done by means of simulation, due mainly to some important drawbacks regarding the investigation with real vehicles. We can have a look at the main differences between simulation and real experiments, as concerns the technologies related to safety in interconnected autonomous vehicles, in Table 2.1.

We can observe that researching in VANETs by means of simulation can be in general more reasonable than performing real experiments in this particular field. Due to the high costs of implementation and the risk which possible test passengers might be subject to makes it necessary to use simulations or recreation models at least at an 
Table 2.1: Comparison between simulation and real experiments

\begin{tabular}{ccc}
\hline Feature & Simulation & Field experiment \\
\hline Costs of implementation & Low & High \\
\hline Flexibility for changes & Very high & Very low \\
\hline Utility of results & Medium & High \\
\hline $\begin{array}{c}\text { Sensitivity to } \\
\text { malfunctions }\end{array}$ & Very low & High \\
\hline $\begin{array}{c}\text { Difficulty of } \\
\text { implementation }\end{array}$ & Medium/low & High \\
\hline Scalability of results & High & Low \\
\hline Spectrum of application & Wide & Limited \\
\hline
\end{tabular}

early stage. Normally, the flexibility provided by simulations in terms of the scope of possible experiments and trials, is much high as that of field experiments, for obvious reasons. The sensitivity to malfunctions is, as can be deduced from previous premises, higher for the cases of real experiments than for simulation. In general, already available simulation platforms contain multiple libraries and protocols that recreate operation in VANET environments very realistically, helping for a rapid implementation of scenarios of evaluation, and allowing for a great scalability of results. The most reasonable advantage of carrying out field experiments when compared to simulation is that the utility of results is more relevant to assess the functionality of the technology in real environments, with real conditions and with real applications. From these facts we can deduce that in general, specially for the investigation in autonomous vehicles, simulation is required at an early stage, covering a remarkable percentage of the dedicated time to research, and leaving the final part to field experiments which serve to validate the technology.

Multiple investigations on simulation approaches and mathematical modeling, of vehicular networks have taken place during the past two decades. Principally coming from the Academia, many research groups have taken an active part in these matters. Among the existing research teams on vehicular networking around the world, the Author would like to highlight the work of the group led by Hartenstein in the Telematics Institute of the University of Karlsruhe in Germany (43), focusing on the systematic 


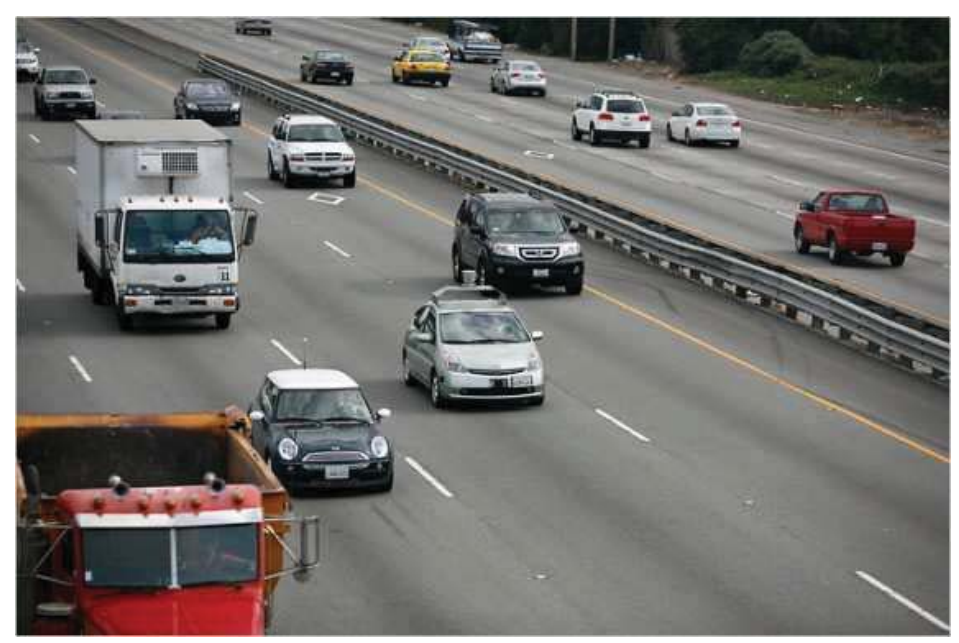

Figure 2.2: Google Car in action

analysis of communication protocols for VANETs, mainly as regards the study of the improvement of traffic safety by the deployment of such technologies. Some other initiatives worth mentioning are the LEGO project in Ireland, aiming at creating in this country a world-leading source of software engineering technologies for the smart cities of the future, and the LaRA project in France, a working proposal made by the french public administration to conduct analyses on the construction of safer roads.

More closely to the experimentation with real autonomous vehicles, we can stand out the initiative of the DARPA Urban Challenge in the USA, which evolved from the previously mentioned initiative (a project originally proposed to evaluate autonomous mobility in military vehicles) to a contest for the investigation of autonomous mobility in civil domains. Actually, the main supervisor of this technology, Sebastian Thrun, professor at the Standford University in the USA, was engaged by Google Inc. to design the currently famous Google Car (see Fig. 2.2). This vehicle has recently obtained a license by the State of Nevada in the USA to drive freely in all roads of this State (13). Gradually, more states in the Union are approving new normatives to allow autonomous vehicles operate on American roads.

In Spain, the framework AUTOPIA, led by the CSIC (Consejo Superio de Investigaciones Cientificas), and directed by $\mathrm{PhD}$. Teresa de Lucio Pedro, from UPM (Universidad Politécnica de Madrid), has carried out an interesting research during the past 15 years regarding autonomous car guiding. Integrating both communications and robotic actuators, this group has obtained interesting results in the particular fields 


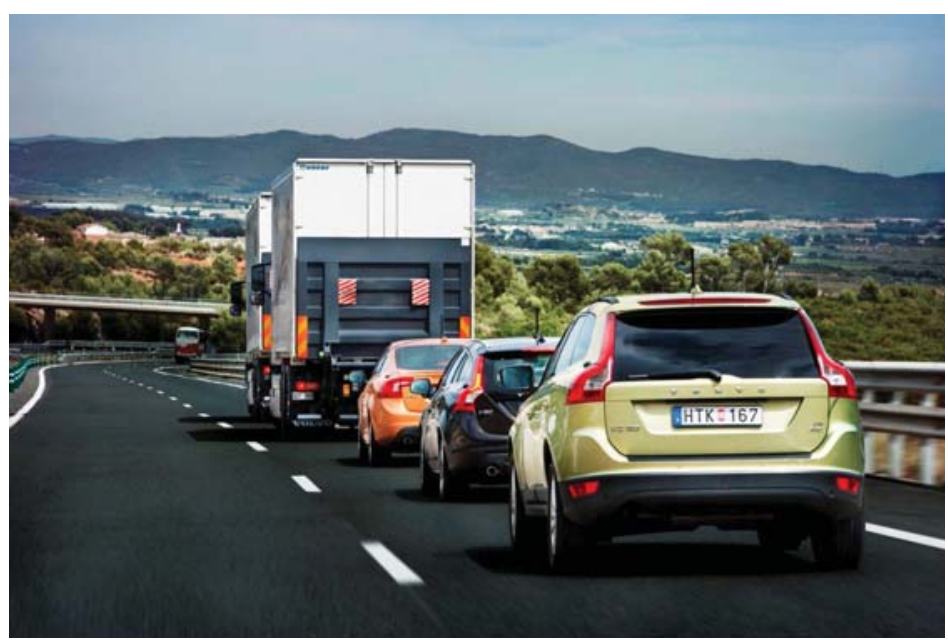

Figure 2.3: Intelligent road train in action (SARTRE project)

of advanced control design, localization, path and speed planning, navigation, vehiclevehicle and vehicle-infrastruture communications for driverless vehicles, see (44). Another very recent project integrating both autonomous driving and communications was led by VOLVO in the european SARTRE project (Safe Road Trains for the Environment) (45), funded by the European Commission under the Framework Program 7. In a few words, this initiative consisted of the design of cooperative strategies in order to allow vehicles to take part of intelligent semi-autonomous platoons on public highways. The key idea was to make a heavy truck responsible of leading a smart road-train in which interconnected vehicles could dynamically take part and let themselves be autonomously guided by reproducing exactly the same route of the leader (see Fig. 2.3). This would allow passengers to carry out activities that in normal conditions would be forbidden while driving: read a book, make a phone call, etc. The most remarkable aspect of this project is the conjunction of both autonomous driving by robotic means and the use of communications to manage the scheme.

In this brief presentation of the main research groups and initiatives that have appeared so far, we have only included the most relevant ones, according to the Author's point of view. For a more in-detail description on these matters please refer to the literature $(7,16,29,41)$. 


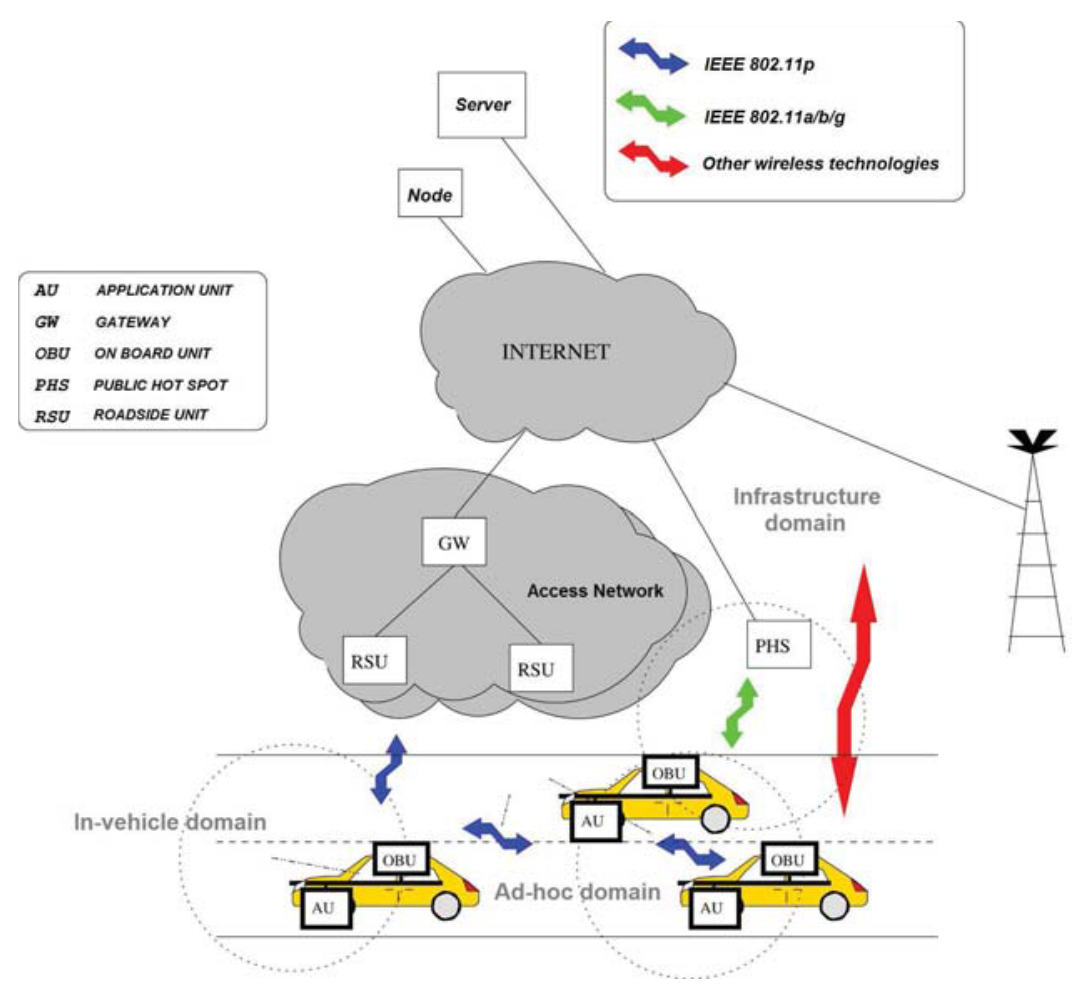

Figure 2.4: Synthesis of a generic V2X environment operating under IEEE 802.11p

\subsection{WAVE IEEE 1609/802.11p, standard for wireless com- munications in VANETs}

Communications in Vehicular Ad-hoc Networks will take place in continuously variable environments, therefore it will be necessary to implement Medium Access Control (MAC) procedures in order for communication protocols to perform properly in such networks. Due mainly to mobility, things get much more complicated, since signal transmissions are heavily influenced by attenuation and scattering. For this purpose, numerous initiatives have been carried out to adequately establish a standardization guideline in order to pave the way for a scalable interoperability that will help carmakers converge to a common framework that will build the efficient, safe and sustainable traffic system of our next future (see Fig. 2.4).

The standard WAVE (Wireless Access for Vehicular Environments) is composed of the family of standards known as 1609.2, 1609.3, 1609.4 and 802.11p (see Figure 2.5). The first three are responsible for defining the administrative level of the system architecture of protocols, while IEEE 802.11 p/DSRC is exclusively concerned with the 
regulation of access to the physical layer. The latter is an extension of the original standard for wireless networks, IEEE 802.11, which serves as a solution to the management of communications in VANETs (actually not originally supported in the standard IEEE 802.11), and therefore takes into account the particular characteristics of these environments: high relative velocities between nodes, short duration of connections, constant handovers and/or signal attenuation losses.

The WAVE amendment, which was finally approved by April 2010, introduces two techniques at the level of medium access control:

1. Reduction of the size of packet headers to strengthen communications, given that latency must be kept very small in such networks and accounting for the high mobility to which vehicles are subject to.

2. Introduction of a priority scheme to distinguish between different application services for providing adapted quality of service (QoS).

\subsubsection{Access methods and operation modes}

The basic scheme which implements the medium access procedure is based on Carrier Sense Multiple Access (CSMA). This mechanism is called EDCA (Enhanced Distributed Channel Access), and is used both for V2V (vehicle-to-vehicle) as well as for V2I (vehicle-to-infrastructure) communications in scenarios of distributed connectivity. It is based on the DCF (Distributed Coordination Function), which was specified for the original 802.11 standard, but integrates service differentiation by defining four access interfaces that implement different backoff schemes (interval of inactivity, i.e. random waiting time until a new transmission attempt is carried out) for transmitting data frames. That is, it makes use of a single networking interface that is shared by four virtual interfaces. As regards general functionality, the operation mode of each interface is identical except for the value of the configuration parameters, as explained below.

Each interface is configured with a series of parameters regarding the maximum window size of inactivity (backoff) and AIFS (arbitrary interframe space) spacing intervals (Fig. 2.6) which are determined by the access class that has been assigned 


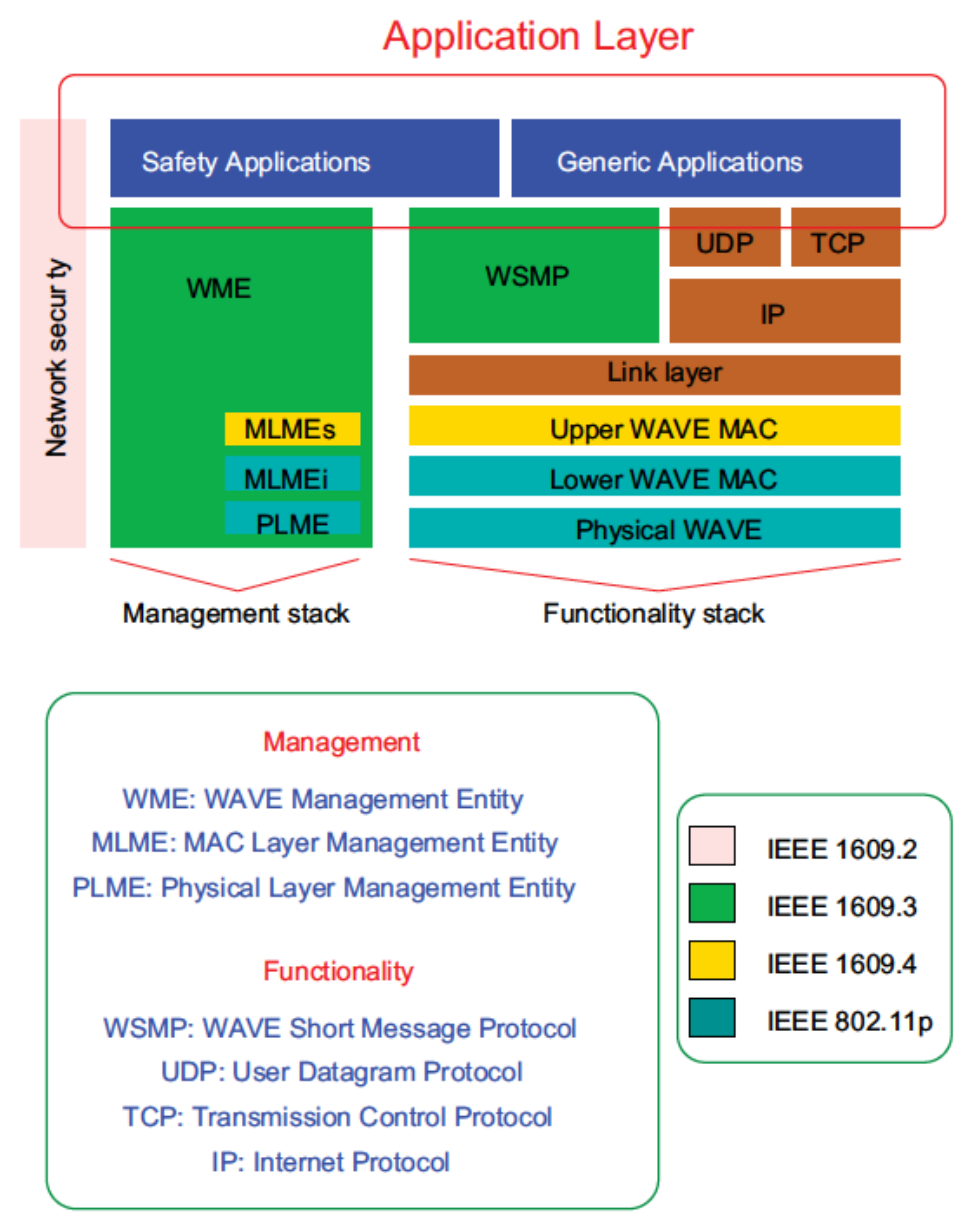

Figure 2.5: Protocol stack for the WAVE IEEE 1609/802.11p architecture

to that interface. This allows for different qualities of service for the many types of applications that have to be served.

According to the main draft standard (8), every application will have an associated value of priority in order to determine the interface to which the corresponding frames will be assigned for a subsequent transmission. Fig. 2.6 shows the mapping process with the eight possible values of priority that can be assigned to packets. The standard also defines a virtual collision manager responsible for handling situations in which two or more packages of different interfaces terminate the idle process (backoff) simultaneously. In this case, the access class with a higher priority will acquire the right to transmit, while the rest will interpret this, at first interblocking situation, as though it had occurred a real packet's collision. This internal scheme, along with the specific 


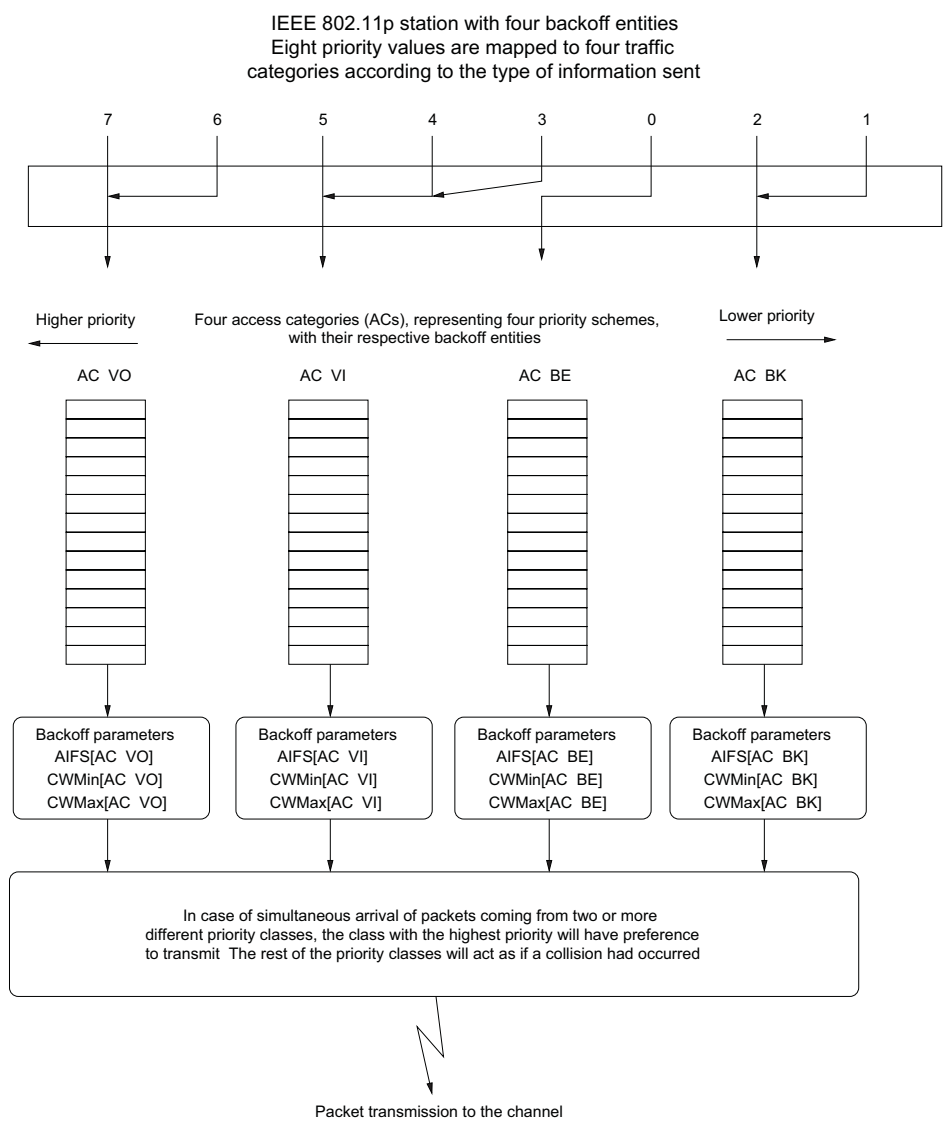

Figure 2.6: Graphical representation of the Medium Access methods of the WAVE IEEE $1609 / 802.11$ p standard

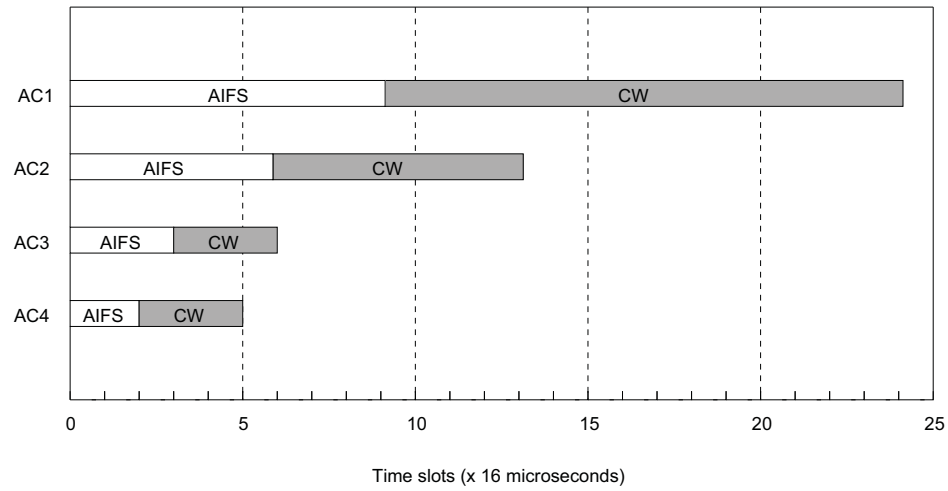

Figure 2.7: Backoff windows' duration in the EDCA mechanism for IEEE 802.11p

configuration of the inactivity period (backoff) of each interface will provide different qualities of service to the user services operating in the application layer. 
Table 2.2: Configuration values for electronic testbench

\begin{tabular}{clllll}
\hline & Exp. $\mathbf{1}$ & Exp. 2 & Exp. 3 & Exp. 4 & Exp. 5 \\
\hline CWMax1 & 7 cycles & 7 cycles & 7 cycles & 7 cycles & 7 cycles \\
\hline CWMax2 & 7 cycles & 7 cycles & 5 cycles & 7 cycles & 5 cycles \\
\hline CWMax3 & 7 cycles & 7 cycles & 4 cycles & 7 cycles & 4 cycles \\
\hline CWMax4 & 7 cycles & 7 cycles & 3 cycles & 7 cycles & 3 cycles \\
\hline txop1 & 7 cycles & 7 cycles & 7 cycles & 3 cycles & 3 cycles \\
\hline txop2 & 7 cycles & 7 cycles & 7 cycles & 7 cycles & 7 cycles \\
\hline txop3 & 7 cycles & 7 cycles & 7 cycles & 15 cycles & 15 cycles \\
\hline txop4 & 7 cycles & 7 cycles & 7 cycles & 31 cycles & 31 cycles \\
\hline AIFS1 & 3 cycles & 5 cycles & 3 cycles & 3 cycles & 2 cycles \\
\hline AIFS2 & 3 cycles & 4 cycles & 3 cycles & 3 cycles & 3 cycles \\
\hline AIFS3 & 3 cycles & 3 cycles & 3 cycles & 3 cycles & 4 cycles \\
\hline AIFS4 & 3 cycles & 2 cycles & 3 cycles & 3 cycles & 5 cycles \\
\hline
\end{tabular}

A good example of this concepts was analyzed by the Author of this Thesis in (46), where a single MAC interface was implemented in an electronic testbench using VHDL $^{1}$. As we can see from Table 2.2, the main aim of this work was to evaluate the performance of the scheme presented in Fig. 2.6 by setting different values for the three configuration parameters of each interface (see Fig. 2.7):

- Inactivity backoff period ( $C W M a x)$. Maximum size of the backoff window for each interface.

- Arbirtrary interframe space (AIFS). Total waiting time before entering the backoff mode.

- Total transmission time ( $t x \boldsymbol{x})$. Total transmission time after winning contention.

In total, five experiments were carried out, whose results are detailed next.

${ }^{1}$ VHDL states for Very High Speed Integrated Circuit in Hardware Description Language, and is used in electronic design automation to describe digital and mixed-signal systems such as field-programmable gate arrays and integrated circuits (47). 


\subsubsection{Experiment 1}

In this first trial, we configured all parameters to be identical (in each particular case) for the four interfaces. At least intuitively, we can predict that the four interfaces would receive the same amount of bandwidth to transmit information. Actually, as can be seen in Fig. 2.8 for Experiment 1, the bandwidth distribution is almost equal for the four interfaces, taking into account that the slight differences between each one correspond to the cases regarding internal collisions (packets ready to be served coming from two or more different interfaces simultaneously), where interfaces with higher priority will win contention against lower priority classes.

\subsubsection{Experiment 2}

In this new test, both the maximum lengths of the contention windows ( CWMax) as well as the transmission lengths (txop) remain fixed, as shown in Table 2.2 for Experiment 2. In this case we only investigate the influence of changing the AIFS parameter dynamically. Fig. 2.8 clearly shows for this experiment that those interfaces with shorter AIFS gain more bandwidth of transmission. Furthermore, in this particular experiment the channel utilization is even worse than in the last case due mainly to the bandwidth shortage to which interfaces with lower priorities are exposed.

\subsubsection{Experiment 3}

Experiment 3 evaluates the influence on bandwidth delivery when the CWMax lengths are modified, keeping the rest of parameters constant. As can be seen in Fig. 2.8 for Experiment 3, having different values for the window length can provide a fairer distribution of bandwidth to the different classes since now the medium access depends on random values to initiate transmissions, respecting the established priority scheme. This is confirmed by the channel utilization which reaches the $80 \%$ of bandwidth profitability, much higher than in the previous case.

\subsubsection{Experiment 4}

When increasing the length duration of transmissions (txop), what we can extract is that the channel utilization reaches its highest value (around $85 \%$ of the total bandwidth), because we use most of the total bandwidth to conduct packet transmissions, 


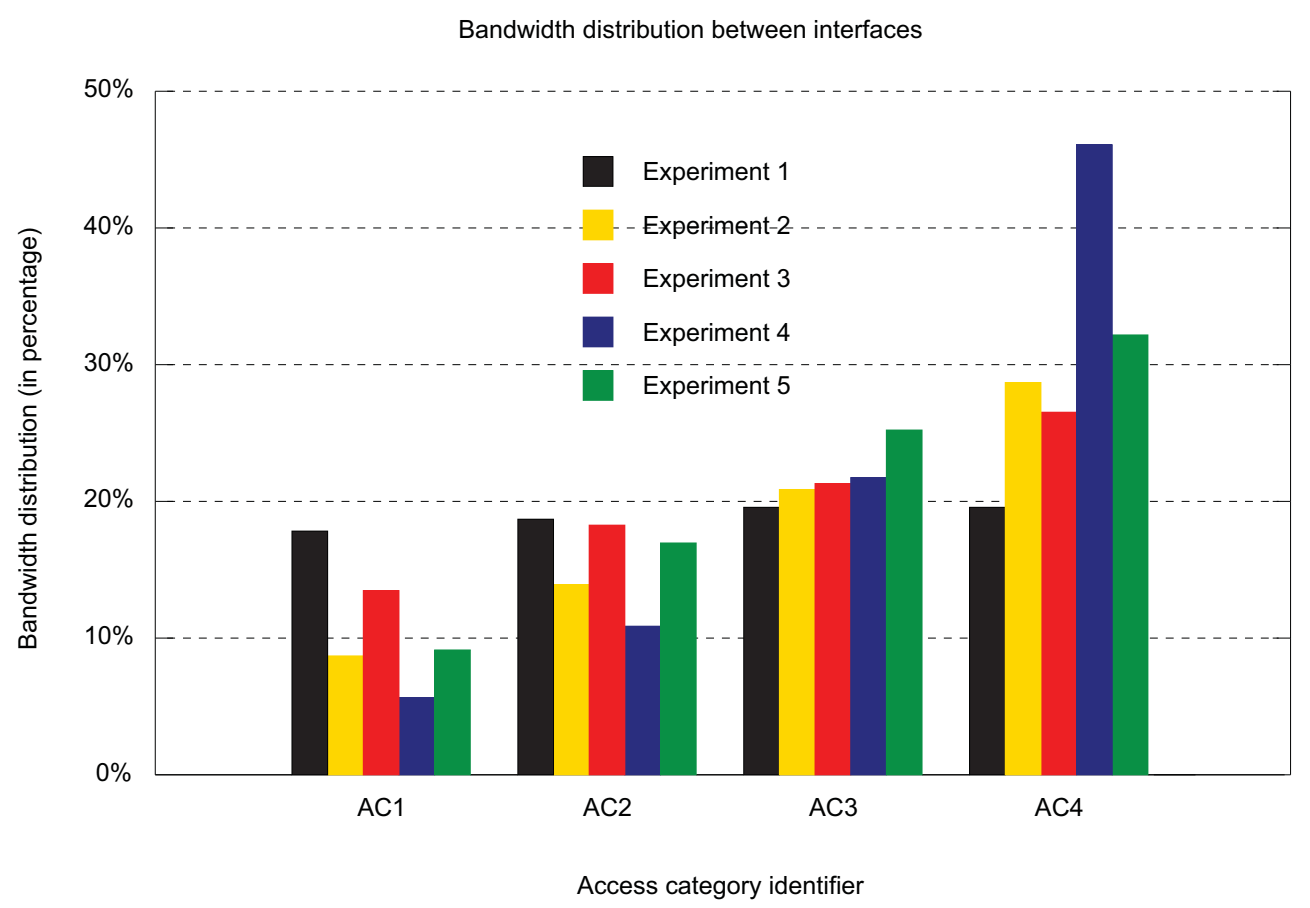

Figure 2.8: Bandwidth assigned to each interface in the different Experiments

minimizing the impact of no-transmissions when using the channel for the AIFS or the backoff procedures. However, priority is intensified here, where we obtain the unfairest distribution of bandwidth among the four classes.

\subsubsection{Experiment 5}

In Experiment 5 we cover the manipulation of all the test parameters, namely $C W M a x$, txop and AIFS, in order to investigate which one has more influence on the bandwidth distribution and channel utilization. We can see from Fig. 2.8 that although AIFS are configured (see Table 2.2) to give more priority to lower classes, the parameter which affects performance the most turns out to be the transmission duration (txop). For this reason, txop will play the major role (with respect to txop and CWMax) in terms of bandwidth distribution, at the expense of worse distributions of the total channel resources for low priority classes.

As could be seen in the previous experiments, the MAC priority classes, and in particular the different parameter setting options, provide a great flexibility to configure the medium access priorities of different types of packets. However we did not count on 
a real environment where there could be multiple simultaneous transmissions. In fact, although results were clearly useful to understand the medium access mechanisms of the standard, we only tested how the internal collision management of a MAC general interface worked. Thus, they would only be realistic for those cases in which a vehicle completely alone, far from urban environments, might communicate to some sort of roadside unit with different types of prioritized information.

In general, the utilization of the channel in a real environment will not reach those values (around 80\%) mainly due to the high number of parallel transmissions that may take place. And of course, if some parameters like the total transmission duration (txop) is too long, some nodes might monopolize the channel usage and not let others to transmit. As a result (and suggested in the standard (8)), connections in VANETs should be as short as possible in order to minimize the impact of these issues. Actually, due to the short longevity of connections in VANETs, vehicles require using lightweight packets to increase reliability and speed up the reception of information. In this regard, this standard implements WSMP ( Wave Short Message Protocol) (48), a network layer protocol that together with the IP Internet protocol share the same layer in the WAVE architecture. WSMP differs from IP in reducing the length and functionality of the packet header. For this reason, the WSM (Wave Short Messages), defined by WSMP packets, are particularly suitable for the transmission of safety information, but can also be used to convey any other type of information.

\subsubsection{Physical layer}

The physical layer is characterized by periodically sending superframes (8) (time slots for transmitting the datagrams). A superframe has a fixed duration of $100 \mathrm{~ms}$, from which the first interval (a default length of $50 \mathrm{~ms}$ ) is used for the control channel (CCH) and the second one for the multiplexing of different service channels ( $\mathrm{SCH}$ ) (Fig. 2.9). Although the Draft suggests the use of default interval durations for these channels, there are also some recommendations on investigating the timing requirements that each single type of application might need, in order to optimize the medium access times for each particular case (8).

As can be seen in Fig. 2.9, due to the unique conditions to be met in VANETs transmissions, the DSRC (Dedicated Short Range Communications) (48) allocated 75 $\mathrm{MHz}$ bandwidth for V2V and V2I communications centering the spectrum exclusively 


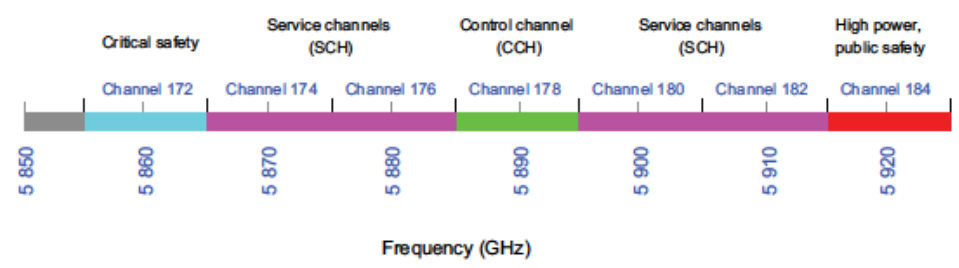

Figure 2.9: Spectral use of the physical layer as defined by the DSRC for VANETs

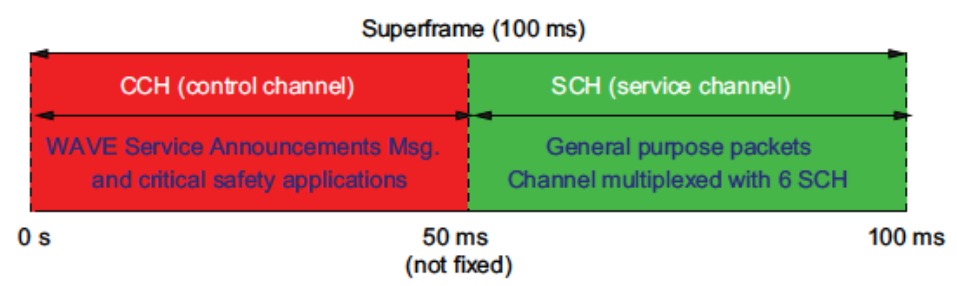

Figure 2.10: Frame structure in DSRC for WAVE

in the $5.9 \mathrm{GHz}$ band (frequencies of ITS - Intelligent Transportation Systems). It also defined four service channels for the transmission of information packets and entertainment (infotainment), and traffic control, and a control channel to issue management packets and safety connections.

Although enormous research efforts have been carried out during the last years either on mathematical modeling, see, for instance, $(49,50)$, as well as on network simulation performance, consult references $(51,52)$ for given examples, of the general WAVE standard, there still remain open some important issues like researching on the design and development of intelligent crosslayer techniques to provide the MAC layer interface with useful information in order to properly establish the settings of the MAC procedure according to the specific requisites of the application layer. Even more important for safety applications (as it is our case) where packet latencies must be minimized in order to allow an acceptable system's performance. In this regard, it is important to conduct a detailed study and classification of applications arising from the use of vehicular networks, in order to clarify what are the needs that different medium access protocols have in order to provide more effective functionality. This is the main question in the next section. 


\subsection{Applications of VANETs}

The design and development of user services for VANETs always has to be preceded by an evaluation phase of the requirements that any application needs to work properly. For example, the service provided by an application on traffic safety management for CCA is not the same as that of an application for Internet data sharing, for obvious reasons. The first one, for example, will have a decisive influence on a limited range of vehicles, consisting normally in a range of entities very close to a leader which launches the application. The latter, on the contrary, might have a spectrum of connectivity that could even reach nodes out of the VANET itself on which the car operates. For this reason, a detailed classification of all the distinctive features of these types of application will serve as an extra source of information for developers to know exactly the requirements of the application they develop.

As a generic list of applications, we can identify first four main categories in which upcoming VANET applications will be classified (according to the perspective of the utility offered to the passengers) (53):

1. Road safety applications: through continuous monitoring of the environment vehicles will send data beacons containing state information about the mobility conditions of the vehicle, such as speed, position, direction, and also event-driven messages to notify other neighbors of possible potential risk under critical circumstances.

2. Applications for driving experience improvement: related mainly to user services that serve to control the characteristics associated to car traffic and its regulation, and personal driving assistance.

3. Infotainment applications: this type of applications will be used to provide advertising information, office-in-car services and entertainment for passengers in the vehicle.

4. Applications of public service information: user services to support public agencies operating under possibly emergency circumstances such as the police or fire departments. 
In order to perform a systematic characterization of the specific user services arising from the previous list, we have also considered the following featuring concepts:

1. Sensitivity versus time. Applications' utility generally depends on the time elapsed since the event to be communicated takes place until the information is received in destination (specially relevant for safety applications).

2. Geographic sensitivity. Applications can be either location-dependent or location-independent, that is, according to the location where they receive the information, its utility will be high or low.

3. Communications scheme. Regarding how the transmission of information packets is triggered, the transmitter vehicle will follow an event-driven communication scheme, or by periodic transmissions (response to the expiration of a timer).

4. Communication partnership. The communication may be established between two vehicles $(\mathrm{V} 2 \mathrm{~V})$, between a vehicle and infrastructure (V2I) or hybrid (V2X), depending on the application context.

In the next subsections we will highlight the different types of applications that are expected to appear in the upcoming VANET networks. We will focus on the technical aspects and requirements arising from each particular type of application, concluding with a table specifying in detail the most important features of each particular application (Table 2.3). This table is based on the classification made in (53) but applying the characterization parameters proposed in the previous lists.

\subsubsection{Active safety applications}

Safety applications are considered the most important group of applications in the context of VANETs (43), and will have a direct impact on road traffic safety when operating in autonomously guided vehicles. This sort of user services are generally characterized by some particular requirements like the need to have very low latencies and high throughputs because the basic principle is to minimize the risk of accident under different traffic circumstances. 


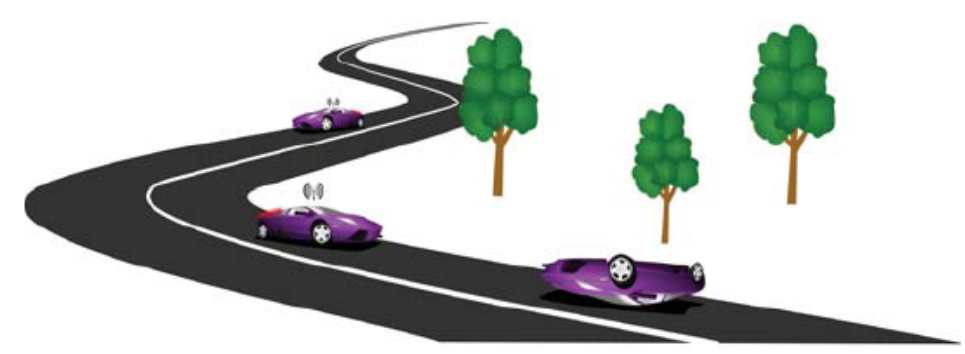

Figure 2.11: Critical emergency brake in which communications are used to provide information to anticipate the accident of a leading vehicle

These applications must generally work in real-time depending on the sort of information to be transmitted, with very small transmission delays (applications of critical risk, such as sudden braking notification (Fig. 2.11), unexpected lane change, etc.), while others may have looser margins in transmission latency (non-critical safety applications such as notification of abnormal conditions of the road at short distances, bad weather, etc.). The morphology of transmission packets should be as simple as possible, avoiding an excess of information that could result in a very large processing delay. However, it is also necessary to use criptography for messages to keep them secure from intentional third-party modifications which might incur into an increased risk of accident. Therefore, there exists a trade-off between message simplicity and complexity for better security of information that still has to be studied in detail.

On the other hand, the utility of safety applications usually have high spatial sensitivity (even more remarkable for safety-critical applications and less critical for non-critical user services). As regards the two possible transmission schemes, safety applications will implement different triggering policies to react to the environment (when considering it necessary), either by reaction to a single event, as a response to the reception of a packet sent by other vehicles, or by reaction to periodically received data, related to any constantly monitored parameter with an anomalous value. We usually associate safety-critical applications to the first policy, since they are related to unexpected situations which the sender might discover and which require to trigger a low-latency process by which neighbors must be timely informed (short-term reaction). In the second case the exchange of periodical information is mainly used to anticipate the possibility of potential risks withing a larger interval of time, i.e. in medium/long term (non-critical safety applications like traffic jams avoidance). Depending on the type of transmission (either if it is event-driven or periodic), app developers have the 


\section{STATE OF THE ART}

complex task of deciding when the notification (event-driven) or reaction (periodic) must take place, since the situation assessment might depend on a wide range of factors that increase the complexity of the decision.

About the type of connectivity, critical safety applications will rely on $\mathrm{V} 2 \mathrm{~V}$ communications primarily, specially for those cases in which complex cooperative decisions have to be made within very short intervals of time. However, it is possible that for this kind of applications the V2I communications' scheme will be also useful to some extent and complement the V2V connectivity with the roadway infrastructure. On the other hand, when talking about non-critical safety applications, the most straightforward solution is to use V2I communications in order to make it possible for a large amount of vehicles to get informed about safety issues that could not be relayed by means of V2V connectivity.

From the perspective of the standardization organisms, we can particularly mention the work of the National Highway Traffic Safety Administration (NHTSA) and the Crash Avoidance Metrics Partnership (CAMP) in the USA (54), which chose, willing to reasonably standardize safety applications by context and applicability differentiation, eight applications as representatives of general safety user services for VANETs: Traffic Signal Violation, Curve Speed Warning, Emergency Brake-lights, Pre-crash Sensing, Forward Collision, Left Turn Assistance, Lane Change Warning, and Stop Sign Assistance. As was previously introduced, the main aim of this Work is to study Cooperative Collision Avoidance schemes under critical situations in which vehicles need to brake or execute evasive maneuvers to avoid colliding with other vehicles or objects appearing on their way. Both Pre-crash Sensing and Forward Collision could be, in this particular case, regarded as Cooperative Collision Avoidance applications, with the specific features in terms of their support on chain collision avoidance, and collision avoidance by active maneuvering.

Due to the very short latencies that intervehicular communications should have in these cases, we assume in all studies regarded here that the type of communication will be V2V (although V2I could also be contemplated, but not in this case). Furthermore, since we consider for all the cases that the risk was caused by an unexpected event, we also assumed that the applications under evaluation work according to an event-driven approach. More details will be shown in the remaining chapters of this Work. 


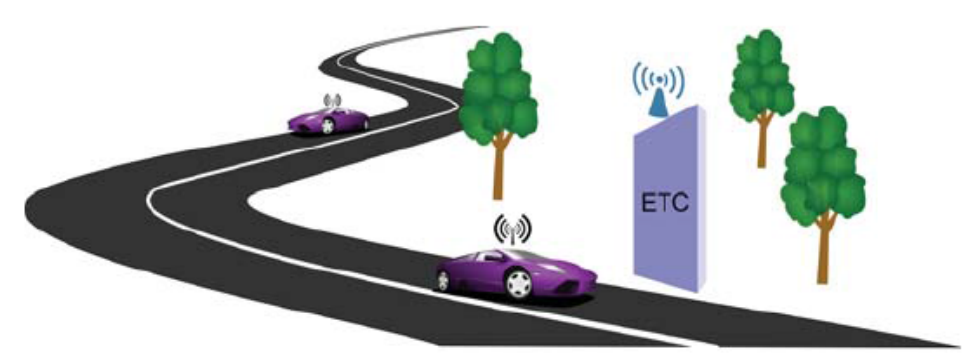

Figure 2.12: Electronic Toll Collection (ETC) by using IEEE 802.11p V2I (Vehicle to infrastructure) communications

\subsubsection{Driving experience enhancement applications}

Driving Management Systems will allow remarkable improvements in both car traffic efficiency as well as in driver assistance services. By car traffic efficiency we mean providing support for actively balancing vehicular traffic accounting for large road networks. This premise applies to everything that influences traffic flows' shaping: ranging from intelligent route planning involving optimal times of arrival until the provision of automatic toll payment services for improving traffic balance on highways (Fig. 2.12). On the other hand, driving assistance systems will serve to assist the driver in making decisions, specially for occasions under which the driver would not be able to react timely.

Traffic control applications are based primarily on vehicle-infrastructure communication schemes, since vehicles periodically send information to the roadway infrastructure about their immediate surrounding conditions, as well as specific information about their motion states. As traffic information is managed on regional (or even higher) levels, data to be transmitted obviously depends on the geographic location of the sender, although the volume of information that must be handled is much bigger than in the case of safety messages. The roadway infrastructure will process and relay this information to faraway processing centers that will in turn make decisions on how to use the received information for the welfare of the whole traffic system. On the other hand, applications of driving assistance will depend essentially on road infrastructure as well as on the own autonomous driving capabilities of the vehicle (actually there are currently brand-new technologies in the market supporting driving assistance, without communications support, like automatic car parking, or road sign visualizer, like it is the case of Opel (55)). 


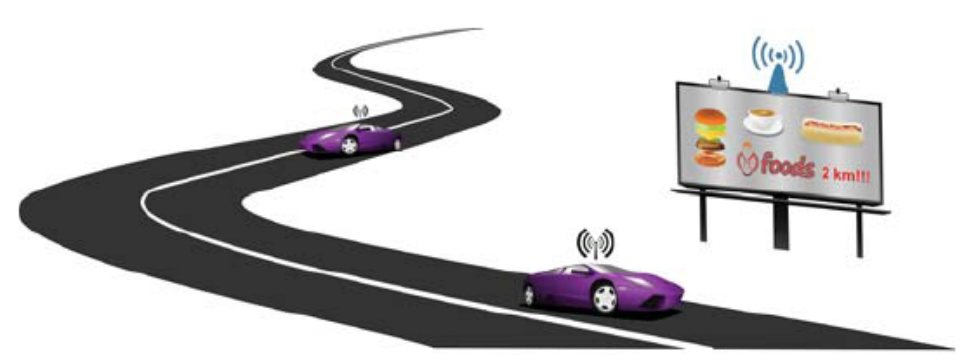

Figure 2.13: Provision of information about menus in a nearby restaurant: possibility to make a reservation before arrival

This type of applications are already present to some extent, but without the use of direct V2I communications as presented in this study. Due to the high costs of deploying a robust network of roadway signs for allowing V2I connectivity, the most probable evolution for this sort of applications is that of $\mathrm{V} 2 \mathrm{~V}$ connectivity in driverless vehicles, with additional connectivity to high performance servers by available technologies like 3G/LTE. This prediction can be justified by taking into account that despite the better functionality of specific roadway infrastructure, using this not-yet deployed technology would require very high costs of deployment. Furthermore, already existing technologies can save this huge costs and give support to this kind of connectivity just allowing IEEE 802.11p and mobile telephony technology to work seamlessly.

\subsubsection{Infotainment applications}

Infotainment applications provide the user with advertising, teletrade services and multimedia content of interest. This sort of applications are characterized by having lower transmission priorities when compared with other safety-related applications like the ones presented before. Moreover, they usually require higher bandwidths to operate, since multimedia and advertising often convey larger volumes of data.

Communications for infotainment applications will be primarily established between vehicles and infrastructure, although V2V schemes could be used in order to complement V2I and increase the coverage extension for cases where roadway infrastructure alone cannot suffice.

Sensitivity to temporal or spatial circumstances is notably reduced in such applications, since in general their usefulness does not depend strongly on time or location. For example, a query sent to the Internet from a vehicular terminal does not normally have to match real-time requirements like safety-related applications do. At the same 


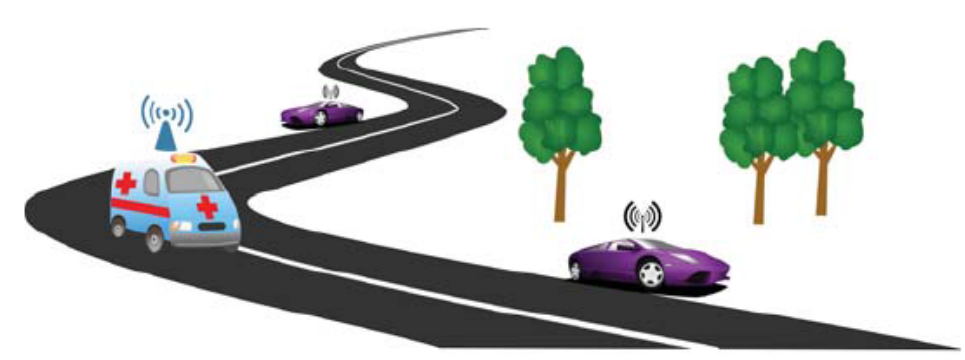

Figure 2.14: Provision of timely alerts from emergency vehicles of the public service

time, the location of the vehicle does not have a decisive influence on the usefulness, although there will possibly be implementations whose functionality will somewhat depend on the location. For example, a restaurant advertising information about its own services and menus, which will be more useful the greater the proximity of the receptor to the restaurant is (Fig. 2.13).

A crucial aspect to note about these applications is the major face possessing a density increase market penetration. Security applications and traffic control rely heavily on technology penetration, since the higher the number of vehicles with this feature, the greater the amount of information we receive to react appropriately to unexpected events. Infotainment applications instead allow from the outset to take advantage of the information provided from roadside access points without the need for a wide array of technology. It is therefore expected that automotive companies are initially inclined towards introducing infotainment applications which consequently induce the inclusion of other applications under the VANET go spreading.

\subsubsection{Emergency service notifications}

Public service applications are normally related to the active notification of alerts associated to the operation or transit of government agencies like ambulances, firemen or the police (Fig. 2.14). It has been considered convenient to distinguish this type of application from the other three possible user service types because although somehow related to safety, it did not originally have the same impact as of safety applications, nor can they be classified as traffic management applications or entertainment.

Regarding temporal and spatial sensitivity these applications could be considered as intermediate, since associated notification packets will in general be transmitted due to events in which higher priority vehicles such as police or ambulance vehicles will take 
part, with low transmission latencies, but not being as demanding as safety applications in these terms. For the geographical position, it all depends on where the originator of the information is located and how far is the destination to which the information must be conveyed.

In these cases, the communication scheme will normally respond to triggering mechanisms (event-driven), since government agencies need only notify their operation when an incident has occurred: fire, theft, accident, etc. The communication is carried out under a V2X scheme (communication between vehicles and vehicle to infrastructure), with $\mathrm{V} 2 \mathrm{~V}$ being of special relevance, since in these cases the public service vehicle on transit should be the one responsible of informing neighboring vehicles, although V2I might also be used for these purposes. 


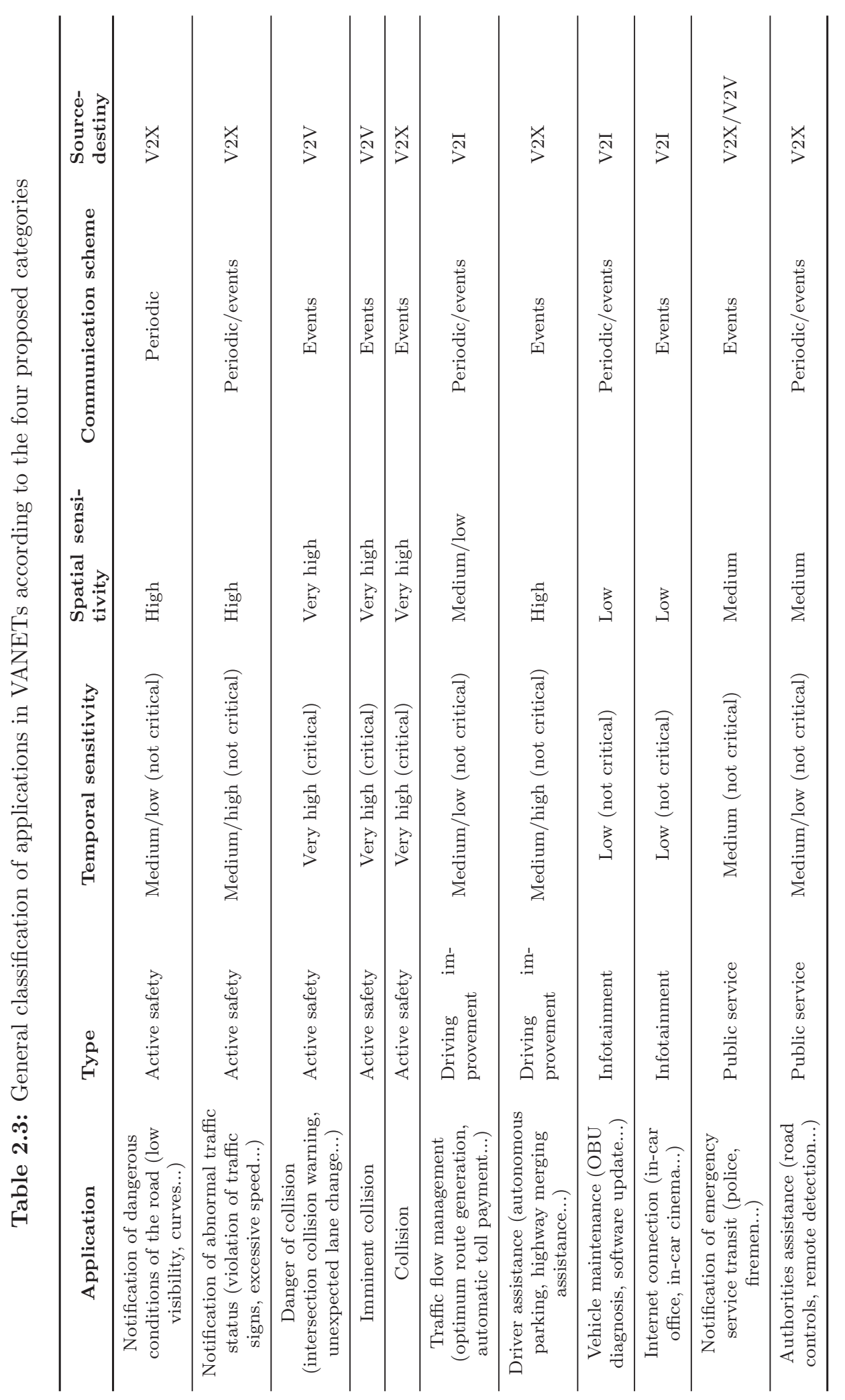


2. STATE OF THE ART 


\section{Part II}

\section{Cooperative chain Collision Avoidance at high speeds}





\section{3}

\section{Simulation and mathematical validation of a CcCA application for VANETs using IEEE 802.11p}

\subsection{Introduction}

We could see in the introductory part of this Book that during the predoctoral stage we centered our attention on the design of active policies to reduce the risk of collision under unpredictable circumstances like the sudden appearance of obstacles on the road. From the two approaches for collision avoidance that we have tackled along the Thesis, in this part of the Document we focus exclusively on CcCA (Cooperative chain Collision Avoidance). In this chapter in particular we present an extensive simulation study on how the implementation of intervehicular communications operating in VANETs can help to reduce the probability of cars getting involved in platoon chain crashes (see Fig. 3.1).

Due to the need to justify the realism of the results obtained by means of simulation, we also decided to propose a mathematical stochastic model which could actually reduce the drawbacks of simulation and at the same time be used as a means of proper validation. We will characterize it by highlighting its main features and testing it under different scenarios to show the correspondence between the simulation approach and the mathematical calculations.

Thus, we propose a CcCA application to evaluate the conditions required to transmit notification alerts in case of emergency deceleration in a platoon of vehicles driving in convoy, either by simulation as well as by the formulation of a mathematical model 


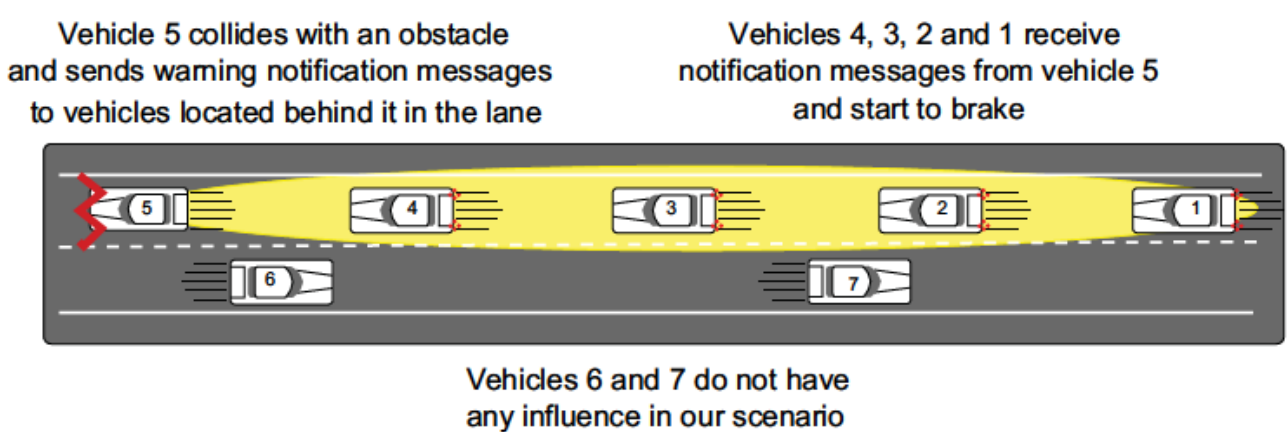

Figure 3.1: Simulation scenario with 802.11 p communication capabilities

that can reduce the complexity of simulations drastically. We show that relay mechanisms are a complementary aid in certain situations in which for a given transmission power, single one-hop delivery schemes are not sufficient to reach all vehicles within the platoon. A deeper analysis on relaying mechanisms is left for the next chapter. On the other hand, improvement over systems without any communication capability as well as other factors like the influence of the human's reaction time are also evaluated to show the detailed behavior of this safety-related application for VANETs. Special attention is also devoted to the evaluation of scenarios where not all the vehicles are equipped with CcCA support. In fact, our results show that when this situation occurs the number of accidents increases compared to a scenario where no vehicle has CcCA support at all. This counterintutive result implies that it is necessary to carefully design the CcCA mechanism, specially in the transition period until this technology becomes completely adopted.

The rest of this chapter is organized as follows. In 3.2 we describe some related work that we found useful to build a state-of-the art idea about CcCA, and compare it directly to the features of our proposal, both in the simulated approach as in the mathematical model. In Section 3.3 we introduce the most important aspects as regards the functionality provided by our application as well as the set of scenarios we chose in order to carry out a performance evaluation of our proposal. Some illustrative results, as well as useful performance metrics complete the set of simulation results obtained in this work. Besides, Section 3.4 will introduce the main characteristics of the mathematical model, with some interesting results that prove the validity of this proposal. Last but not least, Section 3.5 will present some concluding remarks about 
the evaluations conducted in this chapter. Chapter 3 will conclude with a Nomenclature section about the mathematical symbols used for the model ${ }^{1}$.

\section{$3.2 \quad$ Related Work}

Ensuring prompt delivery of emergency brake notifications is crucial for CcCA applications, due to the scarce time that vehicles have to react in this sort of scenarios. Previous simulation experiments on these matters evaluate the performance of different retransmission schemes for CcCA applications. It is the case of (14) where a collision avoidance strategy for intelligent transportation systems is implemented. They propose a vehicle clusterization mechanism based on different parameters such as speed, intervehicular distance, etc. A risk-aware MAC protocol is also designed (based on IEEE 802.11e), in which an emergency level is assigned to every vehicle in the different clusters, and which is used to modulate backoff stages to keep priority differentation for critical applications. However, this service differentiation is already supported in the draft IEEE 802.11p which is used as the MAC layer implementation of our CcCA application. In (17) different broadcast algorithms for emergency notification delivery are assessed to obtain the rate of car crashes under different values of both car and network traffic configuration parameters. A direction-aware protocol and service differentiation between non-safety oriented applications and CcCA are evaluated, as well as the effects of channel errors. They consider a naïve approach where all cars relay the notification as the baseline mechanism. However, we consider that just single transmission should be evaluated first as baseline. In fact, as we show, this simple mechanism performs equally well except for intervehicular distances in the medium range. We will whatsoever analyze the effects of relaying in the next chapter, in order to evaluate under which circumstances notification relaying can be beneficial for the CcCA scheme. As we will see in the corresponding analytical evaluation, we will also restrict our mathematical analysis to the one-hop retransmission policy seen in this chapter. The performance of packet routing mechanisms in this context is evaluated in (15). The routing schemes are pure flooding, OLSR (Optimized Link State Routing) and GAF (Geographic Aware Routing). Surprisingly, pure flooding outperforms the other two routing mechanisms as regards the ratio of delivered packets and average end-to-end delay due to the fact that

\footnotetext{
${ }^{1}$ The Nomenclature appearing here refers exclusively to the mathematical developments of Chapter 3 (please, do not get confused with equally named symbols in Chapter 6).
} 


\section{SIMULATION AND MATHEMATICAL VALIDATION OF A CCCA APPLICATION FOR VANETS USING IEEE 802.11P}

in this method vehicles retransmit everytime a message is received, and consequently the number of relaying nodes is higher and information can reach destination earlier. However flooding generates a significantly large network traffic overload, and for the sake of efficiency in every $\mathrm{CcCA}$ application, it is always important to send as less redundant information to the medium as possible.

Simulated approaches tend to need large computational overheads, implying some inflexibility when carrying out the collection of results. A possible solution to this, although usually more complex, is to derive models which can describe mathematically the problem of interest. Both for the previous reason and for the validation of results obtained by means of simulation, we have developed a rigorous stochastic model which helps us analyze scenarios concerned here with a very high accuracy. Another advantage of our proposal is that we obtain a stochastic model in nature. Actually, collision models for platoons of vehicles can be divided into two main groups: i) Statistical models of the frequency of accident occurrence and their circumstances $(56,57)$, and ii) Models of the collision process based on physical parameters $(58,59,60)$. We can consider our model to fit into the latter group, assuming that we additionally use a communication device that allows vehicles to react earlier to emergency events. In general, models related to the second case are deterministic, implying the need to use stochastic analyses or to execute multiple Monte Carlo simulation runs in order to introduce the randomness that by nature is contemplated in the process. Fortunately, our model is directly stochastic, modeling all physical parameters of the model (such as the intervehicular distance) as exponentially-distributed random variables, as seen in (61). The validation of results is carried out by means of the Monte Carlo approach. Among the previously mentioned works, the reference (58) constitutes an early work (almost a milestone) in the matter (written in 1980), providing a model which analyzes an autonomous train of vehicles by defining a cost function to evaluate the severity of a potential chain collision. Intervehicular spacings are considered fixed and vehicles drive at constant speeds. In (60) we see a similar approach for the collision model, where the motion of a platoon of four vehicles is evaluated to obtain deterministic equations that provide the number of accidents under specific circumstances. Like the model in (58), by randomizing some featuring parameters of the model and running independent Monte Carlo simulations they obtain a statistical study of the problem. The key aspect of our model is, fundamentally, the great generality that it accounts for, providing the 
opportunity to consider all physical variables directly stochastic: intervehicular spacing intervehicular communications' delay, speeds... Besides, the applicability of our model also extends to the qualitative evaluation of the severity of the accidents, as done in (58). All accidental cases reported in that reference, as in this study, are rear-end based. For an exhaustive analysis on head-on collisions, please refer to reference (59), where an extensive physical model of the vehicle is presented (for additional realism on results).

\subsection{Simulations and results}

We will start by the simulation approach and will use the platform NCTUns 6.0 Network Simulator (see Appendix A) to carry out multiple simulation runs to evaluate the different aspects of our $\mathrm{CcCA}$ application. As explained in detail in the referred Appendix, among other interesting issues NCTUns implements the current draft of WAVE (Wireless Access in Vehicular Environments) (48), whose main functionality was introduced in the previous chapter, Section 2.2.

In this section we will present some featuring results characterizing our preliminary CcCA application. First we will make a full description of our scenario under evaluation, and we will conclude this section by giving and discussing some important results on the particular configurations evaluated.

\subsubsection{General scenario description}

The general scenario we want to test consists of a two way road in which vehicles circulate in opposite directions (Fig. 3.1). We concentrate only on one road direction, where cars drive in convoy. When a collision occurs, cars behind the first colliding vehicle react according to one of two possible patterns: starting to brake due to a notification coming from the first vehicle (Fig. 3.1), or beginning to decelerate after noticing a reduction in speed of the car inmediately ahead (Fig. 3.2). In those cases where communications are supported, although either vehicle to vehicle (V2V) as well as vehicle to infrastructure (V2I) communications are valid approaches to implement the VANET in this particular case, for the purpose of this specific study, we will rely only on $\mathrm{V} 2 \mathrm{~V}$ communications to simulate the given functionality. This can be justified because in general it is possible that roadway infrastructure might not cover areas 


\section{SIMULATION AND MATHEMATICAL VALIDATION OF A CCCA APPLICATION FOR VANETS USING IEEE 802.11P}

Table 3.1: Configuration parameters for the simulation scenarios

\begin{tabular}{ccccccc}
\hline Scenario & $\begin{array}{c}\text { No. } \\
\text { vehicles }\end{array}$ & Speed & $\begin{array}{c}\text { Interv. } \\
\text { dist. }\end{array}$ & Tx. power & $\begin{array}{c}\text { Reaction } \\
\text { time }\end{array}$ & $\begin{array}{c}\text { CcCA } \\
\text { Support }\end{array}$ \\
\hline 1 & $31,41,51$ & $30 \mathrm{~m} / \mathrm{s}$ & {$[6-70] \mathrm{m}$} & $22 \mathrm{dBm}$ & $0.2 \mathrm{~s}$ & $100 \%, 0 \%$ \\
\hline $2-\mathrm{I}$ & 31,51 & $30 \mathrm{~m} / \mathrm{s}$ & {$[6-70] \mathrm{m}$} & $22 \mathrm{dBm}$ & $\begin{array}{c}0.2 \mathrm{~s},[0.5-1] \\
\mathrm{s}\end{array}$ & $100 \%$ \\
\hline $2-\mathrm{II}$ & 31,51 & $40 \mathrm{~m} / \mathrm{s}$ & {$[6-70] \mathrm{m}$} & $22 \mathrm{dBm}$ & $\begin{array}{c}0.2 \mathrm{~s},[0.5-1] \\
\mathrm{s}\end{array}$ & $100 \%$ \\
\hline 3 & 31,51 & $30 \mathrm{~m} / \mathrm{s}$ & {$[6-70] \mathrm{m}$} & $22,28 \mathrm{dBm}$ & {$[0.5-1] \mathrm{s}$} & $100 \%$ \\
\hline 4 & 31 & $30 \mathrm{~m} / \mathrm{s}$ & {$[6-70] \mathrm{m}$} & $28 \mathrm{dBm}$ & {$[0.5-1] \mathrm{s}$} & $\begin{array}{c}100 \%, 75 \%, \\
50 \%, 25 \% \\
0 \%\end{array}$ \\
\hline
\end{tabular}

large enough to deliver emergency notifications adequately to all vehicles circulating inside. Relying on an ad-hoc functional scheme for the communications protocol of the proposed $\mathrm{CcCA}$ application is more reasonable since this way it is easier to make sure that at least (if OBUs deployment is sufficiently high, as we will later see) an acceptable percentage of vehicles will receive notifications without any significant problem.

In order to test the worst case situation, cars do not have the chance of modifying their trajectory of movement. Speed is expressed in $\mathrm{m} / \mathrm{s}$, intervehicular distance is varied in steps of two meters, ranging from 6 to 70 meters. Signal transmission power is only accounted for the case with communication capabilities, and takes two values: 22 and $28 \mathrm{dBm}$. For the drivers' reaction time, two cases are studied: one in which vehicles react automatically to the reception of a notification (without any intervention of the driver), and which takes a value of $0.2 \mathrm{~s}$, and the other case, in which after receiving the notification, a driver has to react to this information, thus needing an additional uniformly distributed interval of $0.5-1 \mathrm{~s}$ to start braking. And as an additional parameter we evaluate how the system works when penetration of CcCA technology among the platoon of vehicles reaches only a certain value of deployment $(100 \%, 75 \%$, $50 \%, 25 \%$ and $0 \%$ of the total number of vehicles in the platoon).

The present study is conducted according to two different performance metrics which are described next:

- Percentage of accidents in simulation. In this case, according to a fixed driving speed, the percentage of collided vehicles is represented against different values of the average intervehicular distance parameter. 


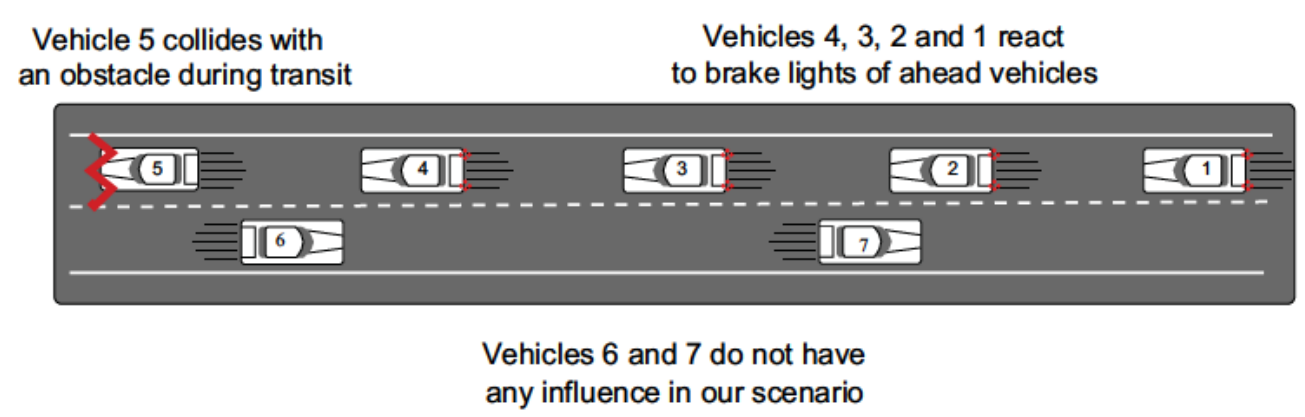

Figure 3.2: Simulation scenario without vehicular communication capabilities

- General merit factor of the system. We measure the quantitative improvement of the system by taking the number of accidents which were avoided using CcCA divided by the total number of collided cars in the system without CcCA and computing the average for all the intervehicular distances.

\subsubsection{Results}

In this subsection results related to four different scenarios are presented and discussed. Simulations are carried out according to a $99 \%$ confidence interval for every case and are performed using a Nakagami fading model, in which we use different values of the Nakagami-m parameter according to the so-called Combined Approach, which has already been shown to recreate more faithfully the influence of the environment on the process of radio propagation in vehicular scenarios (51).

\subsubsection{Scenario 1}

In this first simulation case we compare mainly the functionality of the system with and without the support of communication capabilities, as well as the influence of the total number of vehicles. Table 3.1 shows the rest of the parameters configured for this scenario.

Fig. 3.3 shows clearly that the use of vehicular communications can highly reduce the percentage of collided vehicles. Depending on the transmission power used, not all vehicles in the platoon are able to receive notification messages on time, hence the behavior of central regions in the range 12-35 $\mathrm{m}$ of average intervehicular distance. The behavior of the graph in this region can be explained by those vehicles sufficiently far away from the first colliding vehicle (the sender) which do not receive the accident 


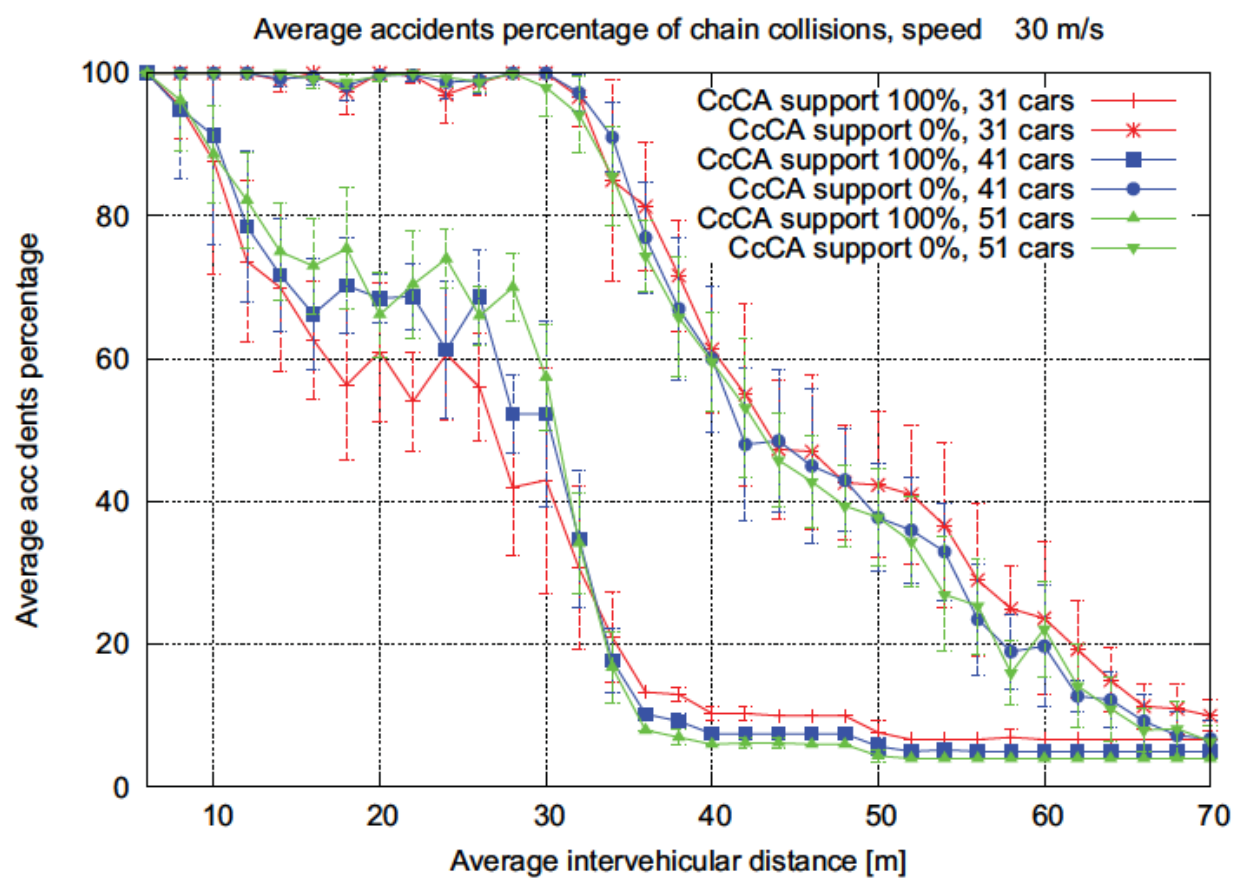

Figure 3.3: Percentage of accidents as a function of the average intervehicular distance (Scenario 1, CcCA vs. No-CcCA)

notification. They cannot start to brake on time and then the percentage of collided vehicles does not decrease so steeply. In the same region, the higher the number of vehicles in the chain, the greater number of collisions, since the number of vehicles which does not hear notifications is also higher. The behavior for the system without CcCA support shows that it is needed an average intervehicular distance of 40 meters to have a reduction of at least the $50 \%$ of accidents. The improvement (around a $50 \%$ for the three cases, Table 3.2) when using the communications' system is then obvious. Summarizing, there is a critical region where relaying becomes necessary, which depends on vehicle clustering (intervehicular distance) and transmission range.

\subsubsection{Scenario 2}

In this second simulation case we evaluate how the system with CcCA capabilities performs when the reaction time to the emergency deceleration (either due to the reception of a notification or because of the brake lights of vehicles ahead) is varied. Simulations compare a scenario of automatic brake reaction (with a latency of 0.2 
Table 3.2: Merit factor comparison for Scenario 1

\begin{tabular}{cc}
\hline $\begin{array}{c}\text { No. } \\
\text { vehicles }\end{array}$ & $\begin{array}{c}\text { Merit } \\
\text { factor }\end{array}$ \\
\hline 31 & $50.29 \%$ \\
\hline 41 & $46.83 \%$ \\
\hline 51 & $45.03 \%$ \\
\hline
\end{tabular}

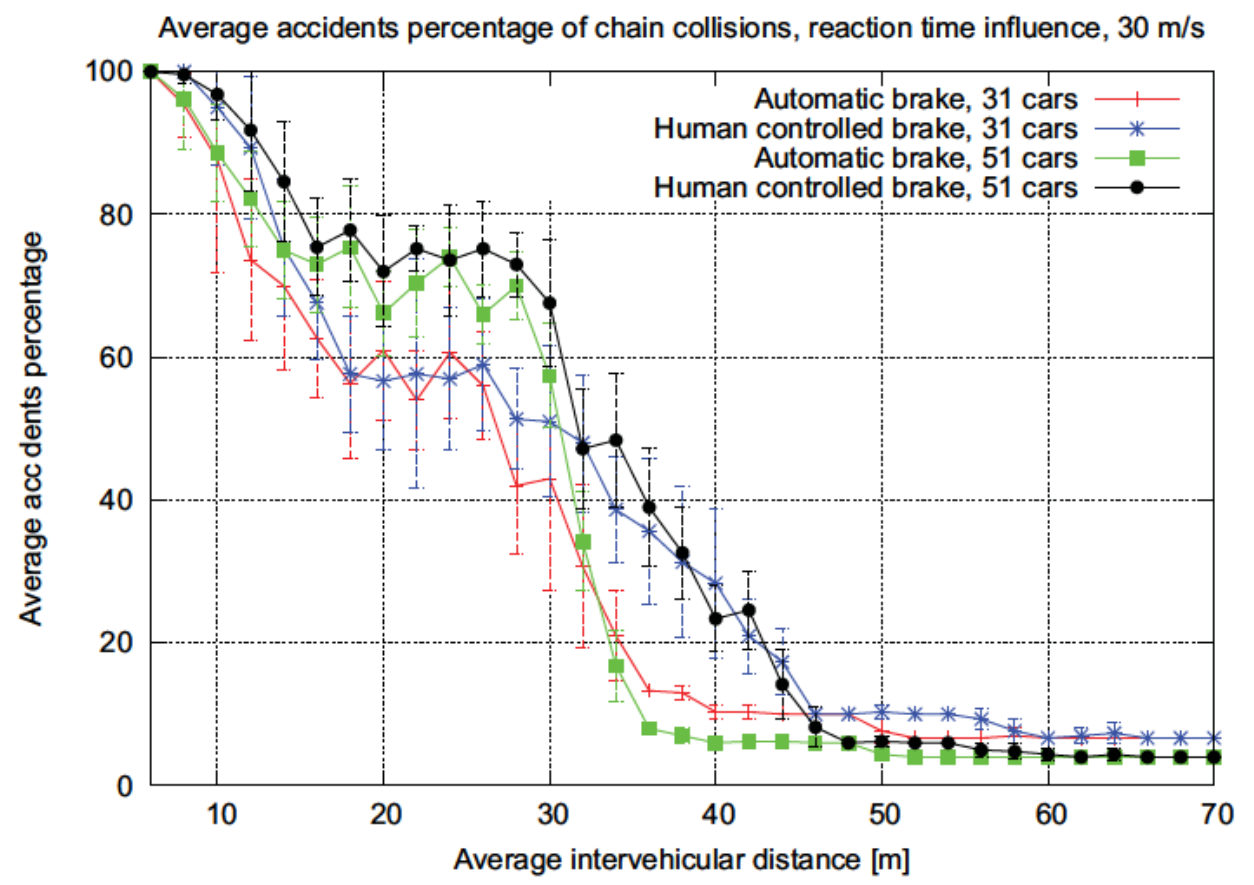

Figure 3.4: Percentage of accidents as a function of the average intervehicular distance (Scenario 2-I, automatic brake vs. human controlled brake, $30 \mathrm{~m} / \mathrm{s}$ )

$\mathrm{s})$ versus human controlled reaction $(0.2 \mathrm{~s}$ plus $0.5-1 \mathrm{~s}$ uniformly distributed human reaction time). The rest of the configuration parameter values is shown in Table 3.1.

Looking at the graphs we can distinguish a region between 0 and $30 \mathrm{~m}$ of average intervehicular distance for the Fig. 3.4 and a similar region between 0 and $40 \mathrm{~m}$ of average intervehicular distance for the Fig. 3.5 in which automatic braking does not imply a significant improvement over human controlled braking. However, in the middle region which takes from 30 till $50 \mathrm{~m}$ of average intervehicular distance in Fig. 3.4 as well as in the region from 40 until $60 \mathrm{~m}$ of average intervehicular distance in 
Table 3.3: Merit factor comparison for Scenario $2(30 \mathrm{~m} / \mathrm{s})$

\begin{tabular}{cccc}
\hline Vehicles & Automatic & Human & Difference \\
\hline 31 & $50.29 \%$ & $41.57 \%$ & $8.72 \%$ \\
\hline 51 & $45.03 \%$ & $33.31 \%$ & $11.72 \%$ \\
\hline
\end{tabular}

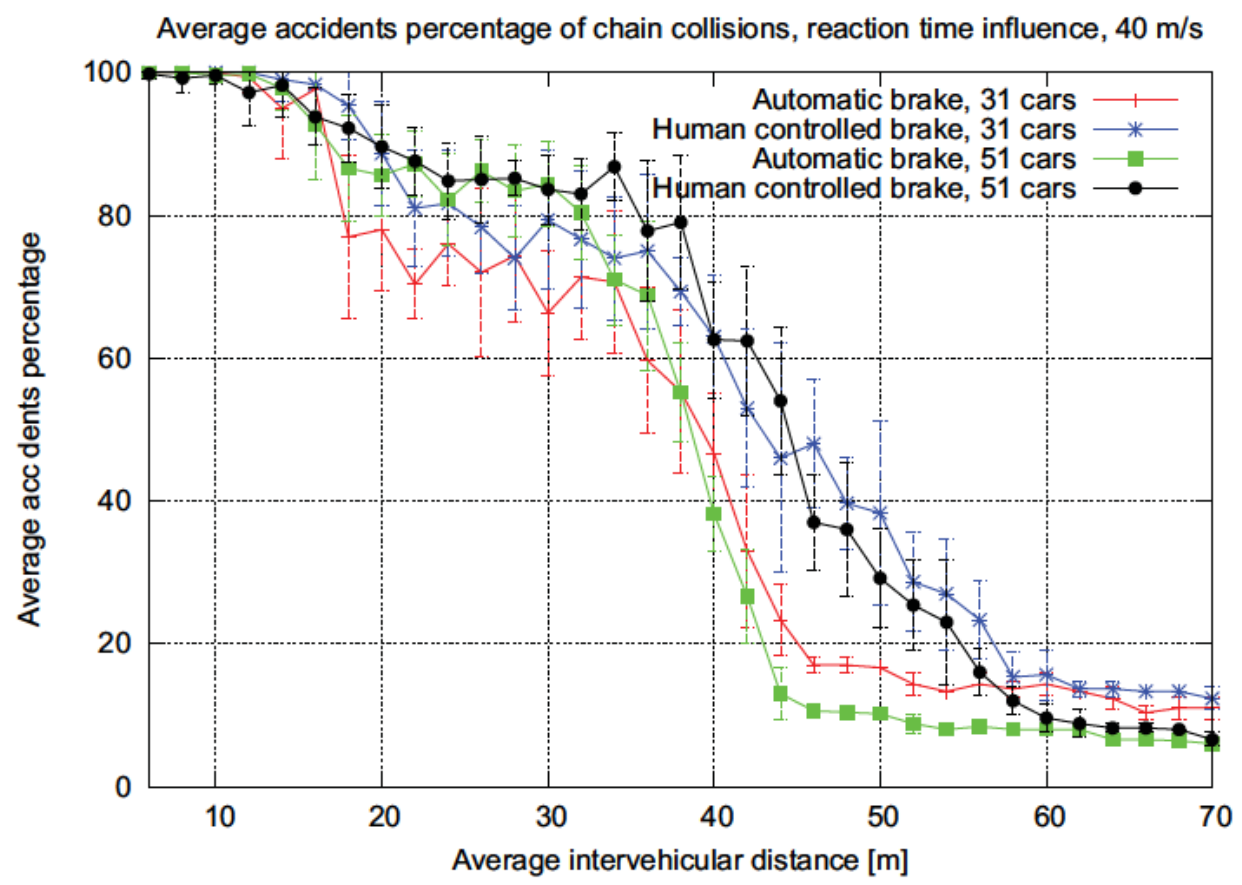

Figure 3.5: Percentage of accidents as a function of the average intervehicular distance (Scenario 2-II, automatic brake vs. human controlled brake, $40 \mathrm{~m} / \mathrm{s}$ )

Fig. 3.5, it is easy to notice that the improvement of automatic braking over human controlled brake is important, due to the fact that for these distances vehicles already have a real chance of stopping without colliding. Thus we can conclude that at middle intervehicular distances the reaction time factor has a major influence over the number of accidents.

In Tables 3.3 and 3.4 we can see the differences in the merit factor of the system for every simulation. We can notice that the higher the speed of vehicles, the worse the merit factor, as can be deduced from the fact that higher speeds require larger distances for vehicles to stop without colliding. Let us remark that these values provide an overall system improvement averaged through all the intervehicular distances. 
Table 3.4: Merit factor comparison for Scenario $2(40 \mathrm{~m} / \mathrm{s})$

\begin{tabular}{cccc}
\hline Vehicles & Automatic & Human & Difference \\
\hline 31 & $40.24 \%$ & $29.05 \%$ & $11.19 \%$ \\
\hline 51 & $37.85 \%$ & $26.45 \%$ & $11.4 \%$ \\
\hline
\end{tabular}

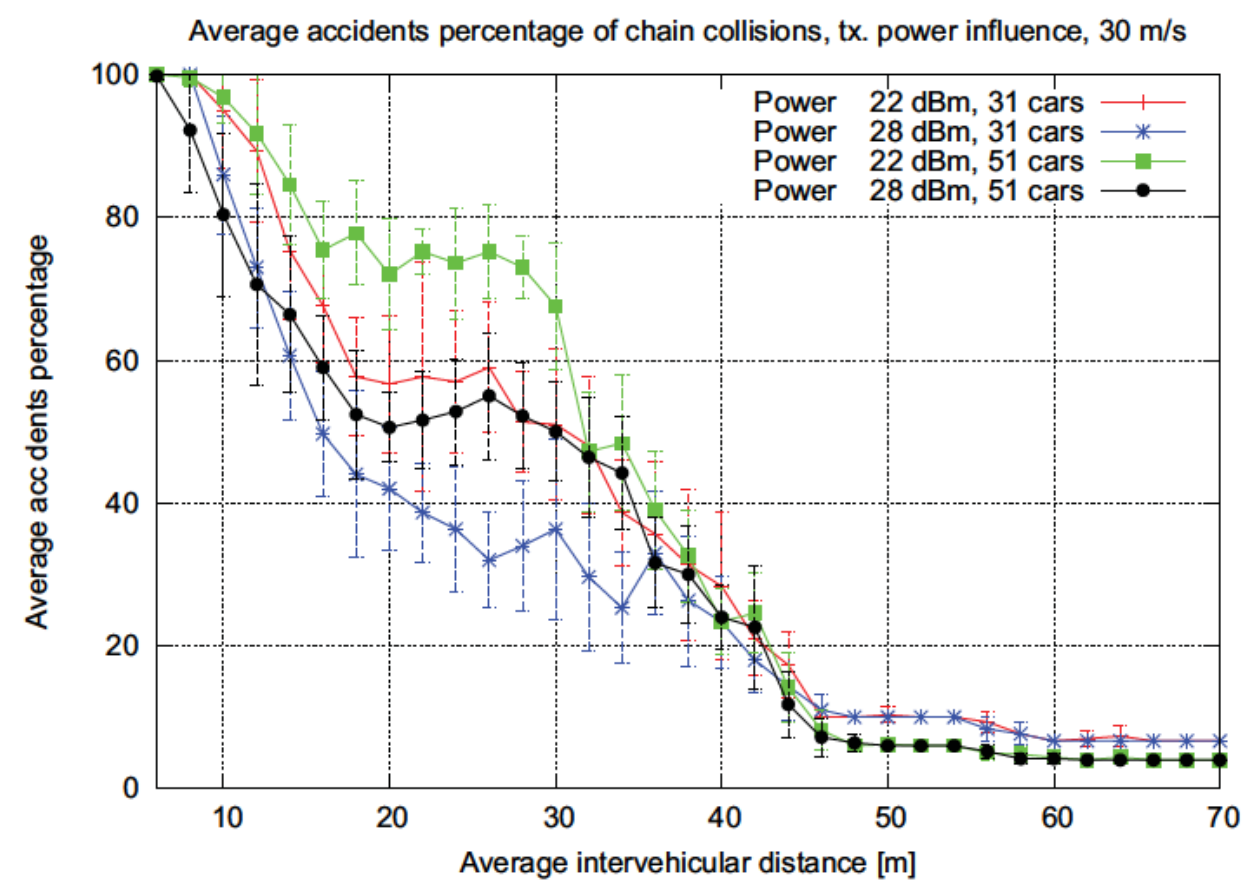

Figure 3.6: Percentage of accidents as a function of the average intervehicular distance (Scenario 3, transmission power influence)

\subsubsection{Scenario 3}

In this scenario we focus on the performance difference when changing transmission power values in our CcCA application. We test the functionality provided by our application by assuming two different transmission power values (22 and $28 \mathrm{dBm}$ respectively). The rest of the configuration parameter values are found in Table 3.1. Fig. 3.6 shows the results obtained.

As can be expected, higher values of the transmission power imply a larger number of vehicles under signal coverage, hence resulting in general in fewer accidents. It is remarkable how there is a certain value for this parameter that provides a considerable reduction in the amount of accidents, and shows what seems more intuitive, that the 


\section{SIMULATION AND MATHEMATICAL VALIDATION OF A CCCA APPLICATION FOR VANETS USING IEEE 802.11P}

Table 3.5: Merit factor comparison for Scenario 3

\begin{tabular}{cccc}
\hline Vehicles & $\mathbf{2 2 d B m}$ & $\mathbf{2 8 d B m}$ & Difference \\
\hline 31 & $41.58 \%$ & $53.08 \%$ & $11.5 \%$ \\
\hline 51 & $33.31 \%$ & $46.33 \%$ & $13.02 \%$ \\
\hline
\end{tabular}

notification must reach a minimum safety distance to minimize the number of collisions. Merit factor (Table 3.5) shows that when transmission power covers a larger number of vehicles it is possible to reduce more significantly the amount of accidents (Fig. 3.6). We conclude here that a retransmission policy, as an additional system, should mostly operate according to the location of vehicles in a convoy in order to further reduce the maximum number of collided vehicles when the transmission power is fixed and cannot reach the whole platoon of cars. Therefore, our evaluation study may serve to feed and tune the appropiate parameters to activate the relay system. Intuitively, from our study, we can realize that this complementary system does not have to be complex and must be designed to minimize the number of safety notification packets provided they travel as long and fast as possible.

\subsubsection{Scenario 4}

In this subsection we evaluate how the system works when the support for CcCA capabilities does not reach the $100 \%$ of deployment among the different cars in the platoon. The rest of the values for the configuration parameters can be found in Table 3.1.

If we compare in Fig. 3.7 the cases with full (100\% of vehicles equipped with CcCA) and null technology penetration ( $0 \%$ of vehicles) versus the other results $(25 \%, 50 \%$ and $75 \%$ of vehicles with CcCA support) we can notice two major features. At first, it is clear that in general for short intervehicular distances (under $40 \mathrm{~m}$ ) the higher the percentage of CcCA support among the convoy of vehicles, the fewer the number of car accidents. This implies that CcCA support is beneficial for low intervehicular distances because in average the number of car accidents is always reduced when the percentage of $\mathrm{CcCA}$ support is increased. However, at longer intervehicular distances, having a mixed compound of vehicles with and without CcCA capabilities (as it would happen at different deployment stages) turns out in a worse behavior of the system which is 


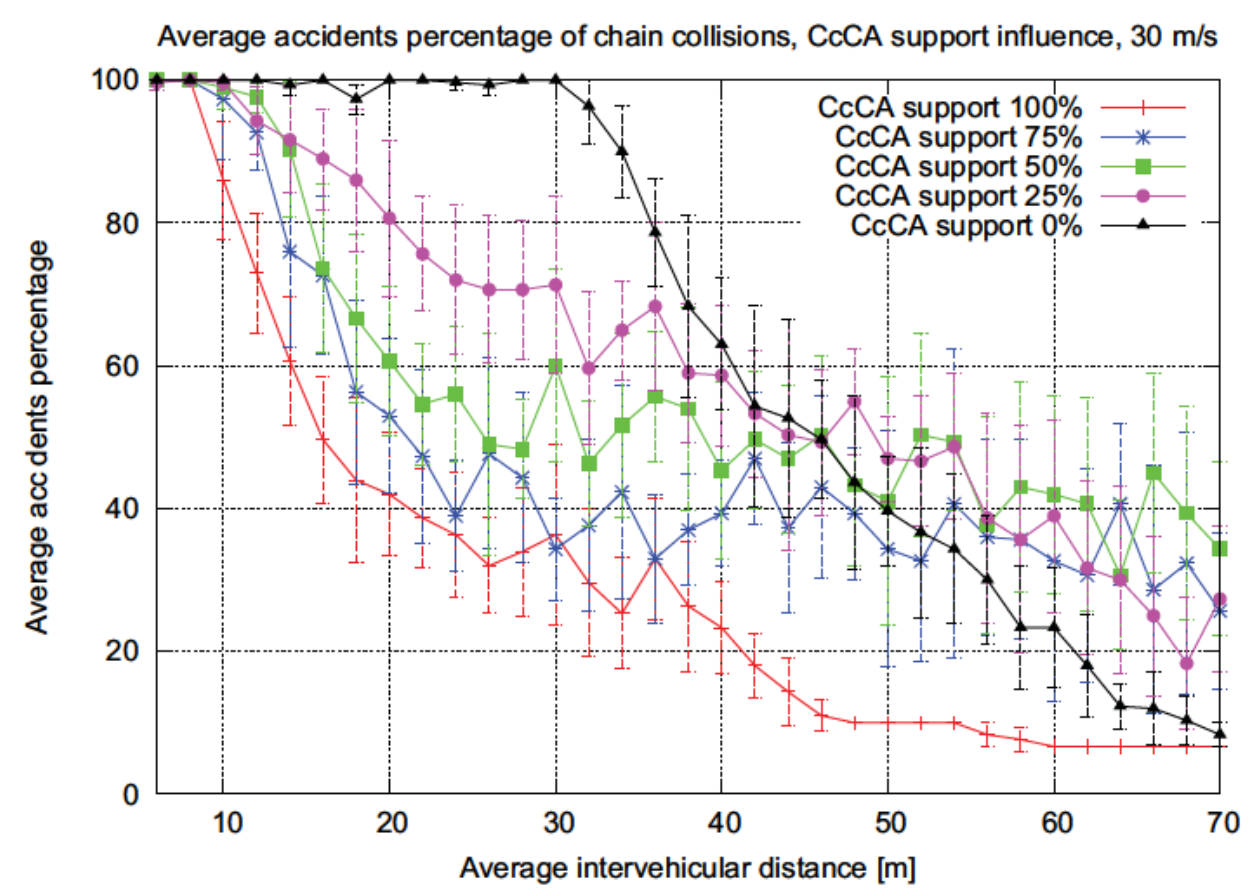

Figure 3.7: Percentage of accidents as a function of the average intervehicular distance (Scenario 4, CcCA support influence)

reflected in the regions at the right side of the graph in Fig. 3.7. In these regions, for $25 \%$ as well as for $50 \%$ and $75 \%$ support, the number of accidents in average is higher than for the case without CcCA capabilities. This behavior can be explained assuming that whenever a vehicle receives a notification or notices a deceleration of its next ahead vehicle, it will always try to stop timely to avoid crashing with its inmediately ahead neighbor. Obviously, this may cause a vehicle to collide with its neighbor behind due to a lack of CcCA support of the following car. Table 3.6 shows that a $25 \%$ of reduction in the number of CcCA-equipped vehicles in the platoon turns out in half the value of the merit factor with respect to full CcCA support (26.36\% versus $53.08 \%$ ). For the other two cases, as we can see, the merit factor is also notably lowered.

We can conclude that, in spite of the simplified scenario evaluated, CcCA may reasonably entail a dangerous risk at these intervehicular distances when full $\mathrm{CcCA}$ support is not possible. This basically would not be a problem if CcCA were not used. For this reason, it is obvious that for a correct penetration of the technology in the market these cases must be fully evaluated and a study of how can be solved 
Table 3.6: Merit factor comparison for Scenario 4

\begin{tabular}{ccccc}
\hline Vehicles & $\mathbf{1 0 0 \%}$ & $\mathbf{7 5 \%}$ & $\mathbf{5 0 \%}$ & $\mathbf{2 5 \%}$ \\
\hline 31 & $53.08 \%$ & $26.36 \%$ & $13.91 \%$ & $6.69 \%$ \\
\hline
\end{tabular}

the apparently problematic and unavoidable transition of null CcCA support to full CcCA support among vehicles with communication capabilities must be necessarily conducted.

\subsection{Stochastic model for CcCA}

In this section, we present the main features of the proposed stochastic model which calculates the probability of accident of vehicles belonging to a platoon in transit on a one-dimensional road (see Fig. 3.8). We will also present some results on the validation of the simulated approach presented earlier, as well as some additional illustrations to show how the model behaves under some particular configurations of the design parameters. At the end of this chapter the reader can also find a Nomenclature section (referring to the model of this section exclusively) where the mathematical symbols appearing in this section are presented for a better comprehension of the concepts treated here.

\subsubsection{Characteristics of the model}

We can observe from Fig. 3.8 that we are dealing in this case with the same scenario as the one evaluated in the simulations of the previous sections of this chapter, but from a mathematical point of view. From the total of $N+1$ vehicles driving in convoy, the first vehicle $C_{0}$ stops suddenly at the initial instant, for example, because of a collision with an obstacle, and automatically sends warning notifications to the following vehicles in the chain. All members in the platoon will support communications, so we assume in the model that all of them will receive information regarding the ahead collision, but after a delay of $\delta_{i}$ seconds that serves as an abstraction of possible effects on the communications' channel. Furthermore each vehicle will start to brake as soon as the notification is received with a constant deceleration $a_{i} \mathrm{~m} / \mathrm{s}^{2}$, independently of the state of the front vehicle $\left(C_{i-1}\right)$. 


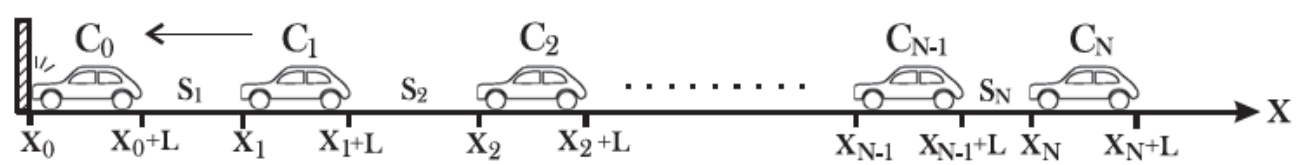

Figure 3.8: The scenario under consideration

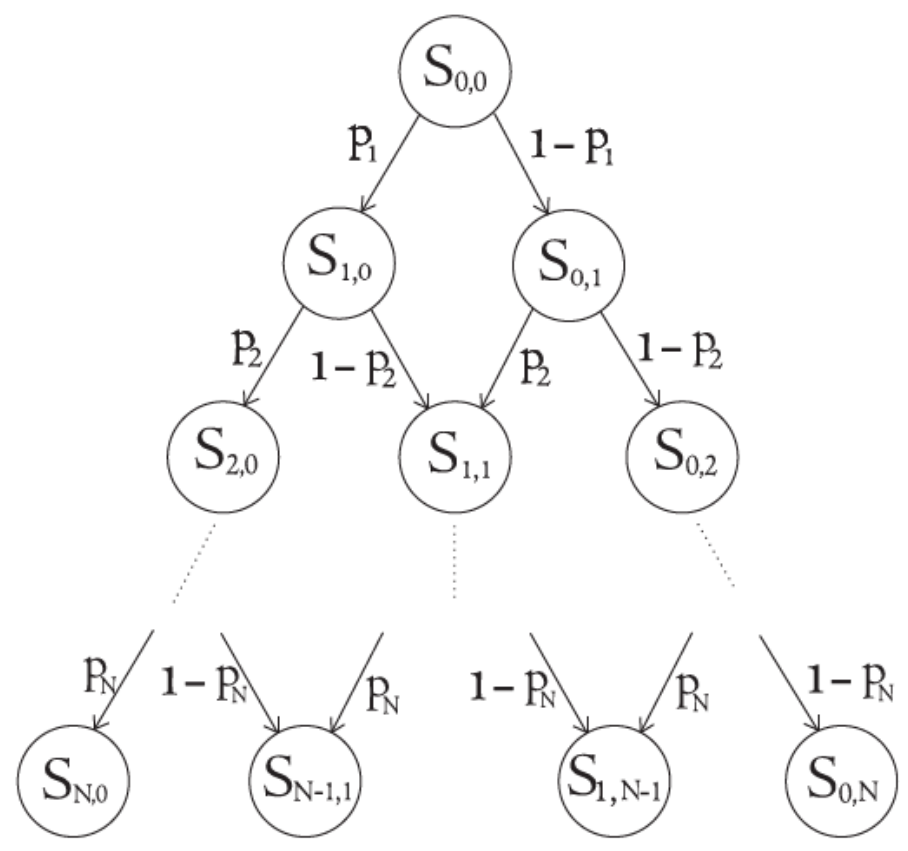

Figure 3.9: Probability tree diagram that defines the model: $S_{i, j}$ represents the state with $i$ collided vehicles and $j$ successfully stopped vehicles

For the sake of simplicity, we assume that every vehicle has the same length $L$ and its position is given by the $x$ coordinate of its front bumper. The leading vehicle stops at coordinate $x_{0}=0$ and the initial intervehicular spacing is $s_{i}=x_{i}-\left(x_{i-1}+L\right)$. We will consider the intervehicular distance $s_{i}$ (see Fig. 3.8) to be modeled by a probabilistic distribution, and other design parameters like the total deceleration $a_{i}$, the notification delay $\delta_{i}$ and the initial speed $V_{i}$ as stochastic variables too. As we did in the last sections, we will represent here the percentage of accidents in the platoon against the average intervehicular distance, ranging from 6 to 70 meters.

The key approach to state this mathematical model is to use a State Markov Chain as the one illustrated in Fig. 3.9 to compute the collision probabilities of each vehicle. Each state in the chain represents the number of collided vehicles $i$ and those that stopped successfully $j$. The first state $S_{0,0}$ corresponds to the initial situation where 


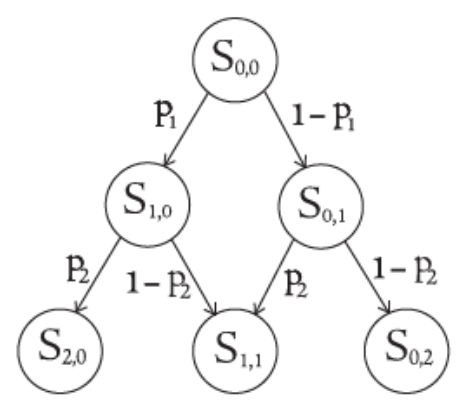

$$
P=\left(\begin{array}{cccccc}
0 & \mathrm{P}_{1} & 1-\mathrm{P}_{1} & 0 & 0 & 0 \\
0 & 0 & 0 & \mathrm{P}_{2} & 1-\mathrm{P}_{2} & 0 \\
0 & 0 & 0 & 0 & \mathrm{P}_{2} & 1-\mathrm{P}_{2} \\
0 & 0 & 0 & 0 & 0 & 0 \\
0 & 0 & 0 & 0 & 0 & 0 \\
0 & 0 & 0 & 0 & 0 & 0
\end{array}\right)
$$

Figure 3.10: Probability tree chain for $N=2$ (3 vehicles) and corresponding state transition matrix

there are no accidents or successful stops. When the first vehicle $C_{0}$ suddenly stops and delivers the critical warning message, the first upcoming vehicle $C_{1}$ may incur into a collision $S_{1,0}$, or stop completely without colliding against $C_{0}: S_{0,0}$. The following vehicle in the chain, namely $C_{2}$, will collide with $C_{1}$ or reach zero speed without colliding. This comes represented by states $S_{2,0}\left(C_{1}\right.$ and $C_{2}$ crash), $S_{1,1}$ (either $C_{1}$ or $C_{2}$ collide, but not the other one), and $S_{0,2}$ (neither $C_{1}$ nor $C_{2}$ collide). From these branches it is possible to derive the rest of possible states of the Markov chain. The important question here is to calculate the transition probabilities $p_{i}$ (probability of collision) that will be used to obtain the percentage of accidents within the platoon for different intervehicular distances according to the probabilistic distributions of the total deceleration $a_{i}$, the notification delay $\delta_{i}$ and the initial speed $V_{i}$ of vehicles.

Let us note how every path in the tree from the root to the leaves leads to a possible outcome involving every vehicle in the chain. The probability of a particular path results from the product of the transition probabilities that belong to the path. Since there are multiple paths that may lead to the same final outcome (a particular leaf node in the tree), the probability of that outcome $\left(S_{i, j}, i\right.$ collided vehicles, $j$ successfully stopped vehicles) will be the sum of the resulting probabilities of every possible path reaching it. As a result, we need to compute the probabilities of going from the initial state to each of the $N+1$ final states in $N$ steps, which are given by $\mathbf{P}^{N}$ (have a look for the case where $N=2$, i.e. three vehicles in the platoon, where the corresponding Markov chain is represented in Fig. 3.10, along with the respective transition matrix). Therefore, the final outcome probabilities are the last $N+1$ entries of the first row of the matrix $\mathbf{P}^{N}$. 


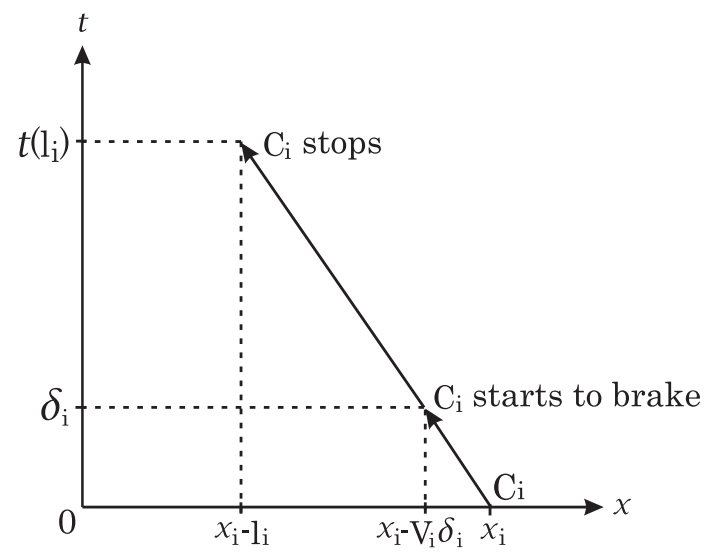

Figure 3.11: Parameters of the kinematic model used to compute the vehicle collision probabilities

Let $\Pi_{i}$ be the probability of reaching the final outcome with $i$ collided vehicles, that is, state $S_{i, N-i}$. Then, $\Pi_{i}=\mathbf{P}^{N}\left(1, \frac{(N+1)(N+2)}{2}-i\right)$. We obtain the average of the total number of accidents in the chain using the weighted sum:

$$
N_{a c c}=\sum_{i=0}^{N} i \cdot \Pi_{i} .
$$

\subsubsection{Computation of the vehicle collision probabilities}

Computing the collision probabilities is the main problem in our model. In this section we start from a deterministic kinematic model and compute the collision probabilities when different parameters of the kinematic model are considered variables. The results are validated by Monte-Carlo simulations. Hence, we start from a basic kinematic collision model provided by (14), that can be summarized as follows.

Let $l_{i}$ represent the total distance traveled by vehicle $C_{i}$ since the emergency event occurs at time instant $t_{0}=0$ until the vehicle completely stops or collides with $C_{i-1}$. Let $\delta_{i}$ be the time lapse that goes between the detection of the emergency event until vehicle $C_{i}$ actually begins to slow down. We call $\delta_{i}$ the notification delay which models the delay between the time instant $t_{0}=0$ and the instant the driver of vehicle $C_{i}$ is aware of it and starts to brake. These parameters are depicted in Fig. 3.11. The notification delay plays an important role if we consider a communication system in operation between the vehicles. In this case, we can assume that the driver starts to brake when it receives a warning message, so if the emergency event occurs at $t_{0}=0$ 


\section{SIMULATION AND MATHEMATICAL VALIDATION OF A CCCA APPLICATION FOR VANETS USING IEEE 802.11P}

the warning message is received at $t=\delta_{i}$ by the vehicle $C_{i}$. However, we consider a more realistic case in which there is also a reaction time before the driver actually starts to brake. Therefore $\delta_{i}=T_{m, i}+T_{r, i}$, where $T_{m, i}$ is the message reception delay and $T_{r, i}$ is the driver reaction time.

Considering a constant deceleration $a_{i}$, the distance needed by vehicle $C_{i}$ to completely stop if it does not collide is given by:

$$
d_{s, i}=\frac{V_{i}^{2}}{2 a_{i}}+V_{i} \delta_{i}
$$

However, when a collision occurs, the actual distance traveled by the car, $d_{c, i}$, is not given by (3.2) anymore, but one has to consider the way the collision has occurred. For example, if a vehicle crashes, its actual distance to stop is obviously shorter than $d_{s, i}$, as illustrated in Fig. 3.12, and also different when both vehicles are still in motion when the crash occurs.

Let us remark at this point that (3.2) implies that a communication system is in place and all vehicles start to brake when they receive the message, independently of the behavior of the preceding vehicles. Otherwise, drivers would start to brake only when they sensed the braking process of its nearest front neighbor as in a car-following approach $(18,62)$, so $(3.2)$ would become a function of the parameters of the preceding vehicle, that is, $d_{s, i}=f\left(V_{i}, V_{i-1}, a_{i}, a_{i-1}, \delta_{i}, \delta_{i-1}\right)$ and the problem would become more complex.

In all the cases the probability of collision of vehicle $C_{i}$ depends on the relationship between its distance to stop, $d_{s, i}$, the total distance traveled by the preceding vehicle, $l_{i-1}$, and the initial intervehicular space, $s_{i}$. That is, when $d_{s, i}<l_{i-1}+s_{i}$ the vehicle is able to stop without colliding.

At this point we also assume another simplification: if two vehicles collide we consider that they instantly stop at the point of collision. This way we keep on assuming a worst case evaluation. There are more realistic approaches, for instance, to take into account the conservation of the linear moments to compute the displacement due to the crash (63).

As can be seen from the previous equation, the number of collisions depends on the vector of velocities $V_{i}$, decelerations $a_{i}$, notification delays $\delta_{i}$, and intervehicular distances $s_{i}$, which we refer to as kinematic parameters. When all the parameters are given, the model is completely deterministic. However, we are interested in a more 


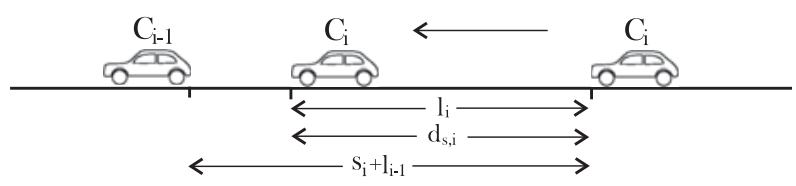

(a) Vehicle $C_{i}$ is able to stop successfully, then $l_{i}=d_{s, i}$

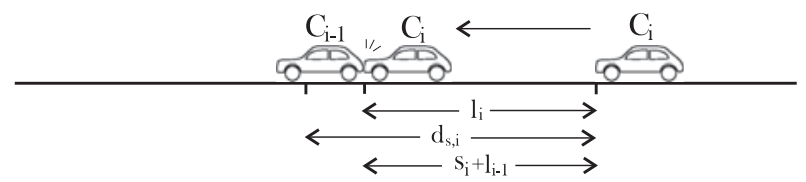

(b) Vehicle $C_{i}$ collides with $C_{i-1}$. In this case, the actual distance covered by $C_{i}$ up to the collision is shorter than $d_{s, i}$ as given by (3.2). Now it is $l_{i}=s_{i}+l_{i-1}$ and depends on the distance covered by $C_{i-1}$

Figure 3.12: The distance $l_{i}$ traveled by a vehicle when there is a collision (b) is shorter than the distance needed by it to stop successfully (a), $d_{s, i}$

realistic case involving random variability of the parameters. To study the influence of the different parameters on collisions we introduce variability on different model parameters as follows: for all the cases we consider that $s_{i}$ is an exponentially distributed random variable with parameter $\lambda$. This parameter represents the density of vehicles on the road, defined as the average number of vehicles per meter. Let us remark that $s_{i}$ can adopt a different distribution and the following model is still valid. The reason for this is that since $s_{i}$ is the intervehicular spacing when the emergency event occurs, we can consider it independent of the rest of parameters of the model, which means that the following equations would be essentially the same, but substituting the exponential probability density function by the corresponding new one. We have selected an exponential distribution because it simplifies the computations and it has been shown that describes well intervehicular spacing when traffic densities are small (61), whereas high traffic densities show log-normal distributions (61).

Once we have described our collision model, we next derive a basic model for the vehicle collision probabilities in which all the parameters are constant except for the intervehicular distance. Then, we extend the model by considering variable the rest of the kinematic parameters. This way we can evaluate the effects of the different parameters on the vehicle collision model. 


\section{SIMULATION AND MATHEMATICAL VALIDATION OF A CCCA APPLICATION FOR VANETS USING IEEE 802.11P}

\subsubsection{Basic model}

Our first step is to evaluate the basic model, considering all the parameters constant, except for $s_{i}$, which is assumed exponentially distributed. If a vehicle is able to stop without colliding and the kinematic parameters are constant it always travels the same distance $d_{s}$. But if there is a collision, a vehicle only travels the initial intervehicular distance plus the distance traveled by the preceding vehicle until it collides. Therefore, we have to compute the collision probability conditioned on the distance traveled by the previous vehicle. In the following subsections we first compute this probability exactly and then we provide an approximation that allows us to simplify the computations when additional variable parameters are considered in the model.

\subsubsection{Case 1. Exact computation of collision probabilities with constant kinematic parameters}

In this case we compute the collision probability exactly. For the sake of clarity, our assumptions are summarized as follows:

1. All vehicles move at the same constant velocity $V$.

2. All vehicles begin to slow down at the same constant deceleration $a$.

3. The delay $\delta$ is the same for all drivers. It implies that all the drivers receive the warning message at the same instant.

Since $V_{i}, \delta_{i}$ and $a_{i}$ are constants, from (3.2) we obtain:

$$
d_{s}=\frac{V^{2}}{2 a}+V \delta
$$

For $1 \leq i \leq N$, the collision probability will be computed as follows:

$$
\begin{aligned}
p_{i}= & P\left(d_{s} \geq l_{i-1}+s_{i}\right)= \\
= & P\left(l_{i-1}+s_{i} \leq d_{s} \mid l_{i-1} \leq d_{s}\right) P\left(l_{i-1} \leq d_{s}\right)+ \\
& +P\left(l_{i-1}+s_{i} \leq d_{s} \mid l_{i-1}>d_{s}\right) P\left(l_{i-1}>d_{s}\right),
\end{aligned}
$$

where $l_{i-1}$ is a random variable that represents the distance traveled by the preceding vehicle (assuming that $l_{0}=0$, since vehicle $C_{0}$ stops instantly at $x_{0}=0$ ), and $F$ is the cumulative distribution function of the exponential distribution, $\exp (\lambda)$, with $\lambda$ the vehicle density (in $v e h / m$ ). 
In this simple case, if vehicle $C_{i-1}$ does not collide then neither does vehicle $C_{i}$, because the velocity, the deceleration and the reaction time are the same for both of them. Moreover, if vehicle $C_{i-1}$ collides, it means that all of the preceding vehicles have collided. From these observations we can conclude that $l_{i-1}=s_{1}+s_{2}+\ldots+s_{i-1} \sim$ $\operatorname{Erlang}(i-1, \lambda)$, and $P\left(l_{i-1}+s_{i} \leq d_{s} \mid l_{i-1}>d_{s}\right)=0$.

Now, we need to compute $p_{i}=P\left(l_{i-1}+s_{i} \leq d_{s} \mid l_{i-1} \leq d_{s}\right) P\left(l_{i-1} \leq d_{s}\right)$.

The joint probability density function of $X=l_{i-1}+s_{i}$ and $Y=l_{i-1}$ is:

$$
g(x, y)=\frac{\lambda^{2}(\lambda y)^{i-2} e^{-\lambda x}}{(i-2) !} \text { for } \quad 0 \leq y \leq x .
$$

So, the joint cumulative distribution function is:

$$
\begin{aligned}
G(x, y) & =\int_{0}^{y} \int_{0}^{t} \frac{\lambda^{2}(\lambda s)^{i-2} e^{-\lambda t}}{(i-2) !} d s d t+ \\
& +\int_{y}^{x} \int_{0}^{y} \frac{\lambda^{2}(\lambda s)^{i-2} e^{-\lambda t}}{(i-2) !} d s d t= \\
= & \frac{\gamma(i, \lambda y)}{(i-1) !}+\frac{(\lambda y)^{i-1}}{(i-1) !}\left(e^{-\lambda y}-e^{-\lambda x}\right), \text { for } 0 \leq y \leq x .
\end{aligned}
$$

where $\gamma$ is the incomplete gamma function, defined as $\gamma(a, x)=\int_{0}^{x} t^{a-1} e^{-t} d t$.

Finally, for $1<i \leq N$ it holds:

$$
\begin{aligned}
& p_{i}=P\left(l_{i-1}+s_{i} \leq d_{s} \mid l_{i-1} \leq d_{s}\right) P\left(l_{i-1} \leq d_{s}\right)= \\
& =\frac{G\left(d_{s}, d_{s}\right)}{F_{y}\left(d_{s}\right)} \cdot F_{y}\left(d_{s}\right)=G\left(d_{s}, d_{s}\right)= \\
& =\frac{\gamma\left(i, \lambda d_{s}\right)}{(i-1) !}+\frac{\left(\lambda d_{s}\right)^{i-1}}{(i-1) !}\left(e^{-\lambda d_{s}}-e^{-\lambda d_{s}}\right)=\frac{\gamma\left(i, \lambda d_{s}\right)}{(i-1) !} .
\end{aligned}
$$

At this point, if the metric of interest is the average number of accidents, the procedure to obtain it is: once we have computed the collision probability for each vehicle, we have to construct the matrix $\mathbf{P}$ described on Section 3.4.1. The next step is to calculate the final outcome probabilities, $\Pi_{i}$, and finally the average number of accidents can be obtained through (3.1).

As can be seen, in this case it is relatively easy to compute the collision probability conditioned on the distance traveled by the preceding vehicle, $l_{i-1}$. However, in the following cases it becomes increasingly difficult. Besides, it can be seen that the collision probability basically depends on the difference $d_{s, i}-l_{i-1}$ of any two cars being 


\section{SIMULATION AND MATHEMATICAL VALIDATION OF A CCCA APPLICATION FOR VANETS USING IEEE 802.11P}

greater than the initial intervehicular distance $s_{i}$. From this observation, and in order to simplify the following computations, in the next section we compute the collision probability using the average distance traveled by the preceding vehicle and compare it with the results of this subsection.

\subsubsection{Case 2. Approximate computation of collision probabilities with constant kinematic parameters}

As discussed previously, in this subsection we compute an approximation to the collision probability for the basic model, where we use the average distance traveled by the preceding vehicle, and compare it with the exact computation of Case 1. For the sake of clarity, our assumptions are summarized as follows:

1. All vehicles move at the same constant velocity $V$.

2. All vehicles begin to slow down at the same constant deceleration $a$ at the same time (the delay $\delta$ is the same for all drivers).

3. We use the average distance traveled by the preceding vehicle to calculate the collision probabilities.

As in Case 1, the distance traveled by a vehicle until it completely stops if it does not collide is given by (3.3).

For $1 \leq i \leq N$, vehicle $C_{i}$ will collide with $C_{i-1}$ if and only if the distance needed by $C_{i}$ to stop is greater than the distance between them plus the average distance traveled by $C_{i-1}, \overline{l_{i-1}}$, so the collision probability of $C_{i}$ is:

$$
p_{i}=P\left(d_{s} \geq \overline{l_{i-1}}+s_{i}\right)=F\left(d_{s}-\overline{l_{i-1}}\right) .
$$

The average distance traveled by a vehicle, $\overline{l_{i}}$, must be computed recursively, starting from $\overline{l_{0}}=0$. For $1 \leq i \leq N$, the average distance traveled by vehicle $C_{i}$ is $\overline{l_{i}}=$ $d_{s}\left(1-p_{i}\right)+d_{c, i} p_{i}$, where $d_{c, i}$ is the average distance traveled by the vehicle in case of collision:

$$
\begin{aligned}
d_{c, i} & =\frac{1}{p_{i}} \int_{0}^{d_{s}-\overline{l_{i-1}}}\left(\overline{l_{i-1}}+x\right) \lambda e^{-\lambda x} d x= \\
& =\frac{1}{p_{i}}\left(\overline{l_{i-1}}+\frac{1}{\lambda}-\left(d_{s}+\frac{1}{\lambda}\right) e^{-\lambda\left(d_{s}-\overline{l_{i-1}}\right)}\right) .
\end{aligned}
$$




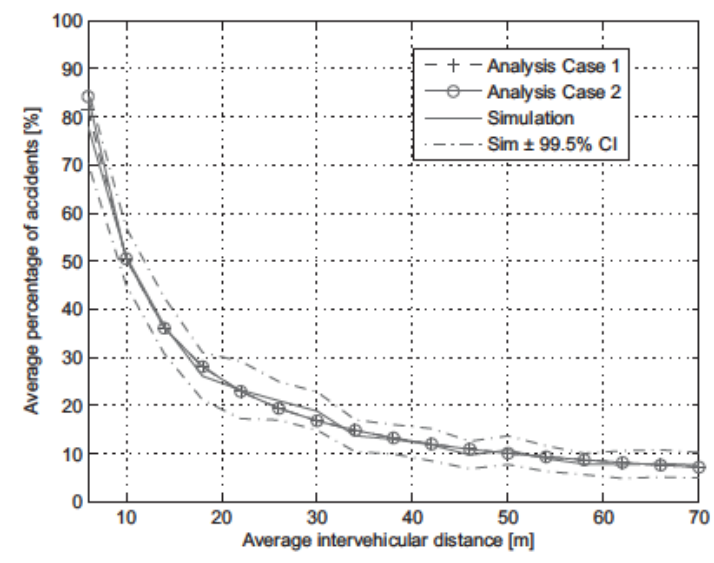

Figure 3.13: Average percentage of accidents versus average intervehicular distance $\bar{s}=$ $\frac{1}{\lambda+L} m$ for basic model, with exact solution (Case 1), approximate solution (Case 2) and Monte-Carlo simulation with a $99.5 \%$ confidence intervals

Then, the equation for $\overline{l_{i}}$ is:

$$
\overline{l_{i}}= \begin{cases}d_{s}\left(1-p_{i}\right)+d_{c, i} p_{i}, & p_{i}>0 \\ d_{s}, & p_{i}=0 .\end{cases}
$$

Now, like in Case 1, we have to construct the matrix $\mathbf{P}$ and calculate the average number of accidents through (3.1).

\subsubsection{Validation and discussion}

Fig. 3.13 shows the results of computing the basic model described in the previous sections. The number of vehicles in the chain is $N=20$, and the rest of the parameters have been fixed at $a=8 \mathrm{~m} / \mathrm{s}^{2}$, which is the maximum deceleration of what is considered as a normal vehicle (17), $V=33 \mathrm{~m} / \mathrm{s}$ and $\delta=T_{m, i}+T_{r, i}=0.1+0.9 \mathrm{~s}$. In this case, $T_{m, i}=0.1 \mathrm{~s}$ is the maximum delay for warning messages that vehicular communication standards specify (48), whereas $T_{r, i}=0.9 \mathrm{~s}$ is an average driver reaction time (64). Fig. 3.13 illustrates the curves for the exact and the approximate basic models. In addition, a Monte-Carlo simulation of the system has been also conducted in order to validate our model, using the platform already presented in Section 3.3 of this Chapter, and described in depth in Appendix A. All the Monte-Carlo simulations in this work have been performed with 10 replications per simulation point and results are shown with 


\section{SIMULATION AND MATHEMATICAL VALIDATION OF A CCCA APPLICATION FOR VANETS USING IEEE 802.11P}

99.5\% confidence intervals. As can be seen using the average distance traveled by the preceding vehicle, $\overline{l_{i-1}}$, computed in Case 2 , provides an excellent approximation to the exact collision probability, since the mean square error between the results of both cases is less than $0.5 \%$. Moreover, simulation results confirm that the model is correct enough, since the mean square error between the results of Case 2 and the Monte-Carlo simulation does not exceed $2 \%$.

\subsubsection{Influence of variability on deceleration, velocity and notification delay}

In this subsection the basic model is extended by considering notification delays $\delta_{i}$, velocities $V_{i}$ and decelerations $a_{i}$ as variables. In most of the cases, they should be considered random variables with their appropriate probability density functions to model some particular effect. At this point, we do not assume any particular probability distribution for them. A discussion on this matter is provided later in Subsection 3.4.4.1.

When deriving a model where all the involved parameters vary simultaneously, several problems arise. Our approach has been to derive a first model considering constant decelerations $a_{i}=a$, and then another one considering constant notification delays $\delta_{i}=\delta$. Later in this section this approach will be discussed and justified in detail.

Therefore let us first consider constant decelerations and variable velocities and notification delays. In this case, the distance needed to stop is neither constant nor equal for each vehicle anymore, but given by:

$$
d_{s, i}=\frac{V_{i}^{2}}{2 a}+V_{i} \delta_{i}
$$

As in Case 1 , for $1 \leq i \leq N$ the collision probability is given by

$$
p_{i}=F\left(d_{s, i}-\overline{l_{i-1}}\right) \text {. }
$$

Again, the average distance traveled and the collision probabilities must be recursively computed. But in this case vehicle collisions may occur in four different ways: (1) vehicles $C_{i}$ and $C_{i-1}$ have not started to brake; (2) only one of them is braking; (3) both of them are braking; or (4) vehicle $C_{i-1}$ has stopped. Each one of these possibilities 
results in a different distance to stop, $d_{c_{1}, i}, d_{c_{2}, i}, d_{c_{3}, i}$ and $d_{c_{4}, i}$, that must be weighted by its probability of occurrence and added to get the average distance traveled $\overline{l_{i}}$ as:

$$
\overline{l_{i}}=d_{s, i}\left(1-p_{i}\right)+d_{c_{1}, i} q_{c_{1}, i}+d_{c_{2}, i} q_{c_{2}, i}+d_{c_{3}, i} q_{c_{3}, i}+d_{c_{4}, i} q_{c_{4}, i}
$$

where

$$
\begin{aligned}
& d_{c_{1}, i}=\frac{1}{q_{c_{1}, i}} \int_{\text {inf }_{1}}^{\sup _{1}} V_{i} t_{c_{1}, i}(x) \lambda e^{-\lambda x} d x, \quad V_{i}>V_{i-1}, \\
& d_{c_{2}, i}=\left\{\begin{array}{lc}
\frac{1}{q_{c_{2}, i}} \int_{\text {inf }_{2}}^{\text {sup }_{2}}\left(V_{i-1} t_{c_{2}, i}(x)+x\right) \lambda e^{-\lambda x} d x, \\
\frac{1}{q_{c_{2}, i}} \int_{\text {inf }_{2}}^{\text {sup }_{2}} V_{i} t_{c_{2}, i}(x) \lambda e^{-\lambda x} d x, \\
d_{c_{3}, i}=\frac{1}{q_{c_{3}, i}} \int_{\text {inf }_{3}}^{\sup _{3}}\left(V_{i} t_{c_{3}, i}(x)-\frac{a}{2}\left(t_{c_{3}, i}-\delta_{i}\right)^{2}\right) \lambda e^{-\lambda x} d x,
\end{array}\right. \\
& d_{c_{4}, i}=\frac{1}{q_{c_{4}, i}} \int_{\text {inf }_{4}}^{\sup _{4}}\left(\overline{l_{i-1}}+x\right) \lambda e^{-\lambda x} d x,
\end{aligned}
$$

and

$$
q_{c_{j}, i}=P\left(i n f_{j} \leq s_{i} \leq s u p_{j}\right)=F\left(\sup _{j}\right)-F\left(i n f_{j}\right),
$$

for $j=1, \ldots, 4$.

The functions $t_{c_{1}, i}(x), t_{c_{2}, i}(x)$ and $t_{c_{3}, i}(x)$ represent the time instants at which the collisions (1), (2) and (3) has occurred, where $x$ is the distance between $C_{i}$ and $C_{i-1}$. The derivation of these time functions as well as the above Eqs. (3.14), (3.15), (3.16) and (3.17) and the appropriate values for the integration limits $i n f_{j}$ and $\sup _{j}$ for $j=1, \ldots, 4$ are provided in Appendix B, Section B.1. A discussion about the circumstances that cause the different types of collision is offered there as well.

At this point we can justify our previous discussed approach: if all the parameters are assumed variable, the number of possible ways in which collisions may occur increases remarkably, and all of them have to be taken into account for the computation of the average distance traveled by a vehicle, as $d_{c_{1}, i}, d_{c_{2}, i}, d_{c_{3}, i}$, and $d_{c_{4}, i}$ in (3.13). This fact makes the resulting equations cumbersome and hard to solve and makes it also difficult to describe the reasons why those events happen and to distinguish the 


\section{SIMULATION AND MATHEMATICAL VALIDATION OF A CCCA APPLICATION FOR VANETS USING IEEE 802.11P}

influence of the different parameters on them. On the contrary, with our approach we can still obtain solutions for most of the cases by computing the solutions of the model for a range of the constant parameter. That is, we can plot a family of curves for the above model varying the deceleration and illustrate another family of curves for the next model varying the notification delay as shown later in Subsection 3.4.5. Moreover, the first case with variable notification delay models a scenario where communications are in use but drivers have control over brake and so a driver reaction time has sense and must be taken into consideration. The second case with constant notification delay exemplifies a scenario where communications are in use and the car is automatically braked as soon as a warning message is received.

Therefore, in the second step we consider $\delta_{i}$ constant. The distance to stop without collision of vehicle $C_{i}$ now includes a variable $a_{i}$ and becomes:

$$
d_{s, i}=\frac{V_{i}^{2}}{2 a_{i}}+V_{i} \delta
$$

Using the same arguments as above, the collision probabilities are given by (3.12).

In this case collisions may occur only according to three different ways: (1) vehicles $C_{i}$ and $C_{i-1}$ have not started to brake; (2) both of them are already braking; or (3) vehicle $C_{i-1}$ has stopped, with their respective actual distances traveled, $d_{c_{1}, i}, d_{c_{2}, i}$ and $d_{c_{3}, i}$. Therefore, the average distance traveled by vehicle $C_{i}$ is given by

$$
\overline{l_{i}}=d_{s, i}\left(1-p_{i}\right)+d_{c_{1}, i} q_{c_{1}, i}+d_{c_{2}, i} q_{c_{2}, i}+d_{c_{3}, i} q_{c_{3}, i}
$$

where $q_{c_{1}, i}, q_{c_{2}, i}$ and $q_{c_{3}, i}$ are given by (3.18), $d_{c_{1}, i}$ and $d_{c_{3}, i}$ have the same form of (3.14) and (3.17) respectively, with slightly different integration limits and time functions which are derived in Appendix B, Section B.2, and $d_{c_{2}, i}$ is expressed by:

$d_{c_{2}, i}=\left\{\begin{array}{l}\frac{1}{q_{c_{2}, i}} \int_{\text {inf }_{2}}^{\sup _{2}}\left(V_{i} t_{c_{2}, i}(x)-\frac{a_{i}}{2}\left(t_{c_{2}, i}(x)-\delta\right)^{2}\right) \lambda e^{-\lambda x} d x, \quad V_{i}>V_{i-1}, a_{i-1}-a_{i}=0, \\ \frac{1}{q_{c_{2 a}, i}} \int_{\text {inf }_{2 a}}^{\sup _{2 a}}\left(V_{i} t 1_{c_{2}, i}(x)-\frac{a_{i}}{2}\left(t 1_{c_{2}, i}(x)-\delta\right)^{2}\right) \lambda e^{-\lambda x} d x \\ \quad+\frac{1}{q_{c_{2 b}, i}} \int_{\text {inf }_{2 b}}^{s u p_{2 b}}\left(V_{i} t 2_{c_{2}, i}(x)-\frac{a_{i}}{2}\left(t 2_{c_{2}, i}(x)-\delta\right)^{2}\right) \lambda e^{-\lambda x} d x, \quad a_{i-1}-a_{i} \neq 0 .\end{array}\right.$

Note that in all the cases the above equations provide additional meaningful information since we have derived the probability of the different ways in which vehicle 
collisions may occur $\left(q_{c_{j}, i}\right)$ as well as the average distance traveled by the vehicles $\left(d_{c_{j}, i}\right)$. This information can be used, for instance, to design a measure about the severity of collisions that assigns different weights to every particular type of collision. Finally, one may wonder whether it is possible to adopt a simpler approximation, instead of the average distance $\overline{l_{i}}$ to compute the probabilities, or whether there is significant difference between the $\overline{l_{i}}$. Indeed, for vehicles located in the chain far enough from the leading vehicle, $\overline{l_{i}}$ are closer to eq. (3.2), that is, the influence of the outcome of the previous vehicle is weak and the outcome depends mainly on the particular values of the parameters. However, for vehicles close to the leading vehicle, there is a strong dependence on the outcome of the previous vehicle and Eqs. (3.13) and (3.20) cannot be neglected.

\subsubsection{Validation and discussion}

The next stage would be to assign the kinematic parameters and notification delays appropriate values that model realistic scenarios. As an example, in order to take into account an underlying communication model, the notification delay should be assumed to be a random variable with an appropriate probability density function. In this way, information packet collisions in a heavily loaded shared communications channel can be modeled with an appropriate random variable for the access delay and characterized also by $T_{m, i}$ (65). Furthermore, since vehicles move at different speeds, the velocity should be assumed to be a random variable too. Let us note that, in most of the practical cases, intervehicular distances and velocities represent the state of the system when the incident occurs, and so they should be considered random variables, though determining their distributions and ranges require a proper characterization of the scenario of interest. Accelerations and delays can be controlled by different means after the incident, and so depending on the application evaluated they can be considered constant or assigned particular values. 


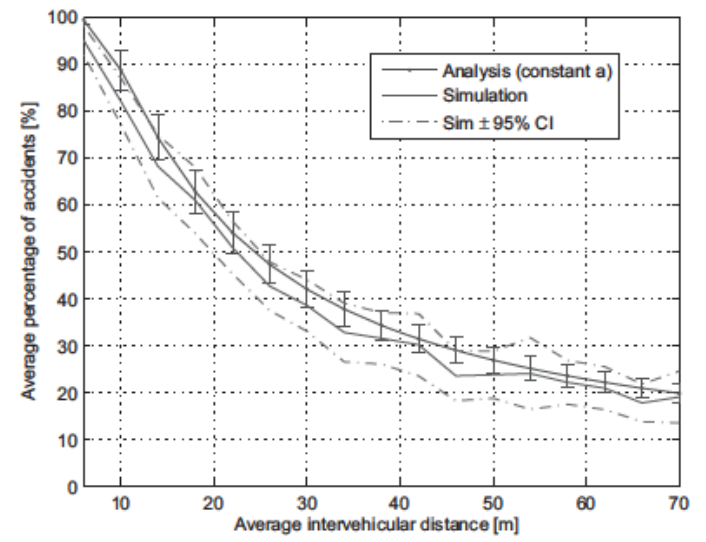

(a) $\delta_{i} \sim U(0.5,1.5) \mathrm{s}, V_{i}=33 \mathrm{~m} / \mathrm{s}$ and $a_{i}=8 \mathrm{~m} / \mathrm{s}^{2}$

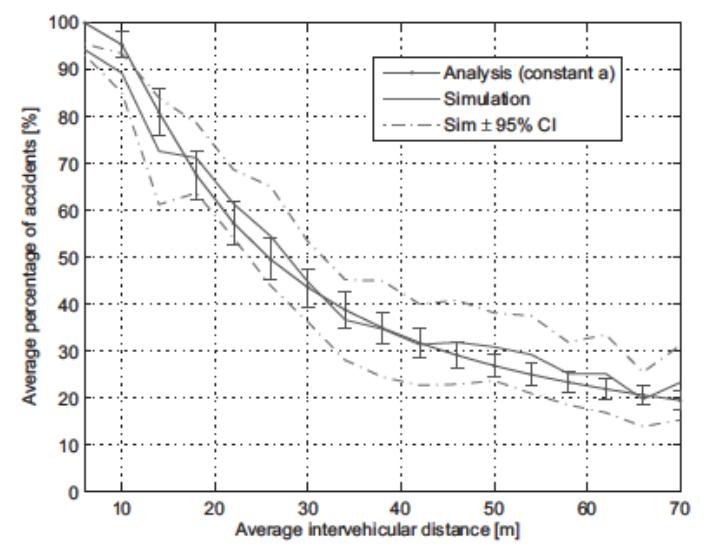

(b) $\delta_{i}=1 \mathrm{~s}, V_{i} \sim U(30,36) \mathrm{m} / \mathrm{s}$ and $a_{i}=8 \mathrm{~m} / \mathrm{s}^{2}$

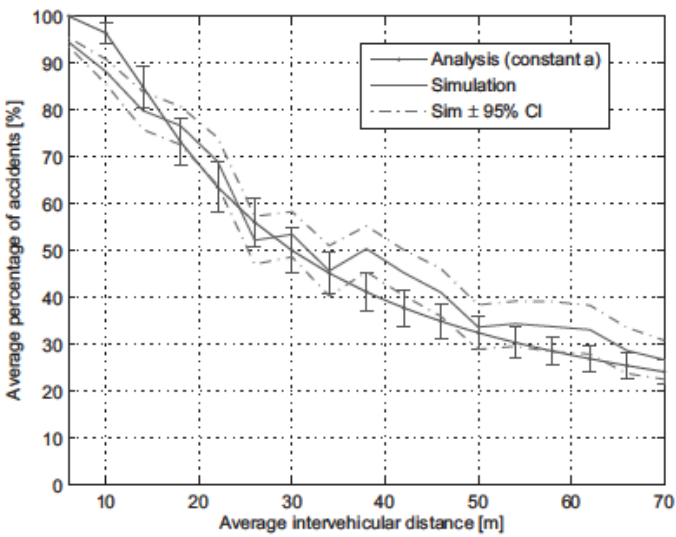

(c) $\delta_{i} \sim U(0.5,1.5) \mathrm{s}, V_{i} \sim U(30,36) \mathrm{m} / \mathrm{s}$ and $a_{i}=8$ $\mathrm{m} / \mathrm{s}^{2}$

Figure 3.14: Validation of the model with constant deceleration through the evaluation of three different scenarios. 


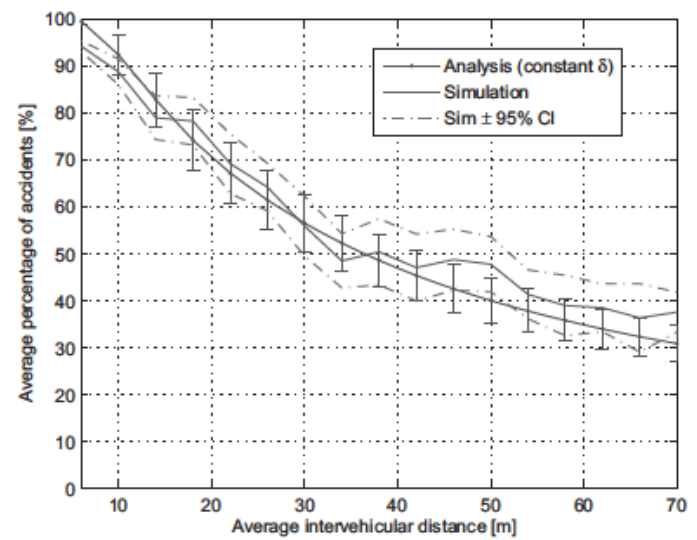

(a) $\delta_{i}=1 \mathrm{~s}, V_{i}=33 \mathrm{~m} / \mathrm{s}$ and $a_{i} \sim U(4,8) \mathrm{m} / \mathrm{s}^{2}$

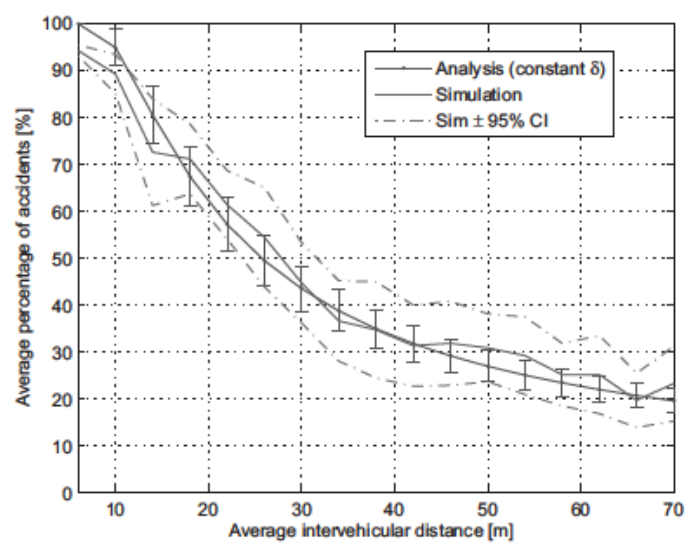

(b) $\delta_{i}=1 \mathrm{~s}, V_{i} \sim U(30,36) \mathrm{m} / \mathrm{s}$ and $a_{i}=8 \mathrm{~m} / \mathrm{s}^{2}$

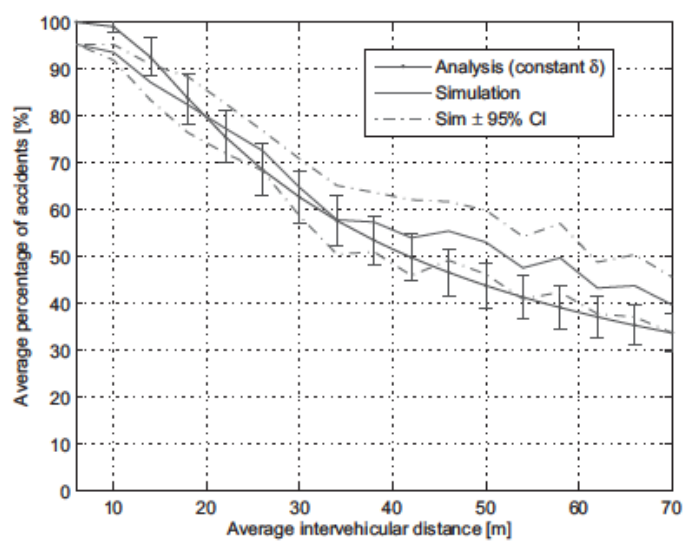

(c) $\delta_{i}=1 \mathrm{~s}, V_{i} \sim U(30,36) \mathrm{m} / \mathrm{s}$ and $a_{i} \sim U(4,8) \mathrm{m} / \mathrm{s}^{2}$

Figure 3.15: Validation of the model with constant notification delay through the evaluation of three different scenarios 


\section{SIMULATION AND MATHEMATICAL VALIDATION OF A CCCA APPLICATION FOR VANETS USING IEEE 802.11P}

However, with regard to the analysis, introducing additional random variables makes it hard to obtain a closed-form solution for the collision probabilities, even for the simple case when parameters are assigned uniformly distributed random variables, and the benefits are not clear. Therefore, the solutions when a parameter is a random variable have been computed numerically. The parameters are supposed to be uniform random variables and Eq. (3.1) has been computed 100 times and averaged. In all the cases we assume a chain of $N=20$ vehicles.

A solution for the model with constant deceleration has been computed for three different scenarios. In the first one, $\delta_{i}$ is assumed to be a uniform random variable ranging between 0.5 and $1.5 \mathrm{~s}$, whereas the velocity has been fixed at $V=33 \mathrm{~m} / \mathrm{s}$. In the second scenario, $V_{i}$ is assumed to be a uniform random variable between 30 and 36 $\mathrm{m} / \mathrm{s}$ and the notification delay has been fixed at $\delta=1 \mathrm{~s}$. In the last simulation, both the velocity and the notification delay are assumed to be uniform random variables ranging between 0.5 and $1.5 \mathrm{~s}$ and between 30 and $36 \mathrm{~m} / \mathrm{s}$, respectively. In all the simulations the deceleration is kept constant at $8 \mathrm{~m} / \mathrm{s}^{2}$.

The validation of the model with constant notification delay has been also done for three different scenarios. In the first one, deceleration $a_{i}$ is assumed to be a uniform random variable between 4 and $8 \mathrm{~m} / \mathrm{s}^{2}$, whereas the velocity has been fixed at $V=33$ $\mathrm{m} / \mathrm{s}$. In the second one, $V_{i}$ is assumed to be a uniform random variable between 30 and $36 \mathrm{~m} / \mathrm{s}$ and the deceleration has been fixed at $a=8 \mathrm{~m} / \mathrm{s}^{2}$. In the last simulation, both the deceleration and the velocity are assumed to be uniform random variables between 4 and $8 \mathrm{~m} / \mathrm{s}^{2}$ and between 30 and $36 \mathrm{~m} / \mathrm{s}$, respectively. In all the simulations the notification delay is kept constant at $1 \mathrm{~s}$.

Finally, in order to validate the results for our solutions, the corresponding MonteCarlo simulations in NCTUns have been conducted as well.

Figs. 3.14 and 3.15 show the results of this section. Let us remark that these pictures are provided to validate that our model shows correctly the dynamics of the system. A discussion on the influence of the parameters on the collision process is deferred to the next Section. The average number of accidents computed with our model for each of the six cases is compared with the aforementioned Monte-Carlo simulations. The standard deviation has been computed and shown as errorbars. Dashed lines show the $95 \%$ confidence interval of the corresponding simulation. In all the cases, the results reasonably confirm the validity of our model, even using $\overline{l_{i-1}}$ as approximation, since 
the mean square error between the results of the analysis and the simulation remains between $3.5 \%$ and $6 \%$ for all the cases.

\subsubsection{Applications and discussion of the model}

Once our model has been validated, we use it to evaluate the influence of the different parameters on the vehicle collision process. In this section we present some results as an example of the utility of our model. The metric used here is the average percentage of accidents in the chain, but the model could also provide information about the probability of collision or the average distance traveled for the different manners a collision can occur. A systematic evaluation of different scenarios as well as different metrics is out of the scope of the study on CcCA of the present Chapter, and left as possible future worklines. First, we focus on qualitative aspects of the influence of the parameters on the collision process that our model can quickly reveal. Then, we discuss quantitative aspects of the results provided in this section.

As for the qualitative evaluation, we first provide a set of figures that show the influence of the different parameters. Fig. 3.16 shows a family of curves for both instances of the model over a range of their constant parameter, $a$ or $\delta$. As can be seen in Fig. 3.16(a), the number of accidents is clearly sensitive to the deceleration capabilities of the vehicles, which agrees with the results obtained in (58). However, it does not seem to be statistical difference for different notification delays when the deceleration and velocities are variable. This result is also in accordance with (58), where it is shown that moderate changes in the notification delay cause small variations in accident severity. Later in this Subsection we discuss when the delay actually has an important influence on the number of accidents. 


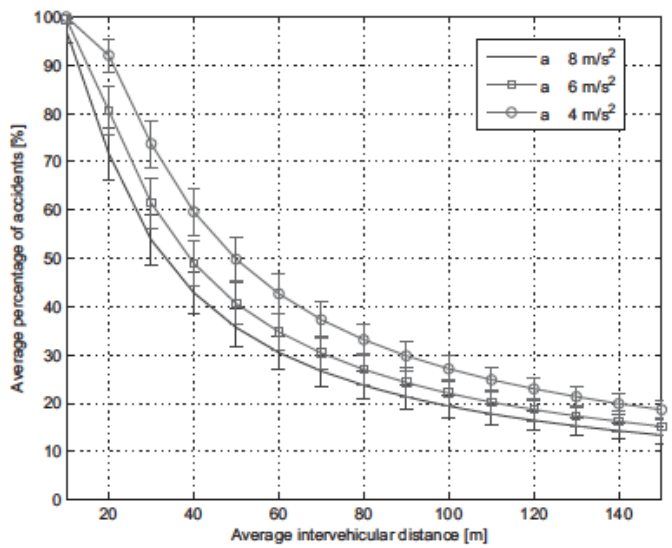

(a) Average percentage of accidents for different decelerations when $V_{i} \sim U(30,36) \mathrm{m} / \mathrm{s}$ and $\delta_{i} \sim U(0.5,1.8)$

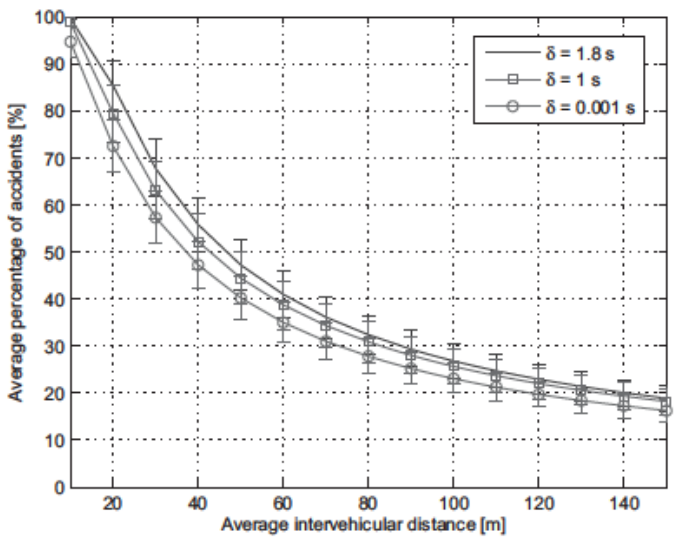

(b) Average percentage of accidents for different notification delay when $V_{i} \sim U(30,36) \mathrm{m} / \mathrm{s}$ and $a_{i} \sim U(4,8)$ $\mathrm{m} / \mathrm{s}^{2}$

Figure 3.16: Performance of the model with different constant decelerations (a) and message reception delay (b) 


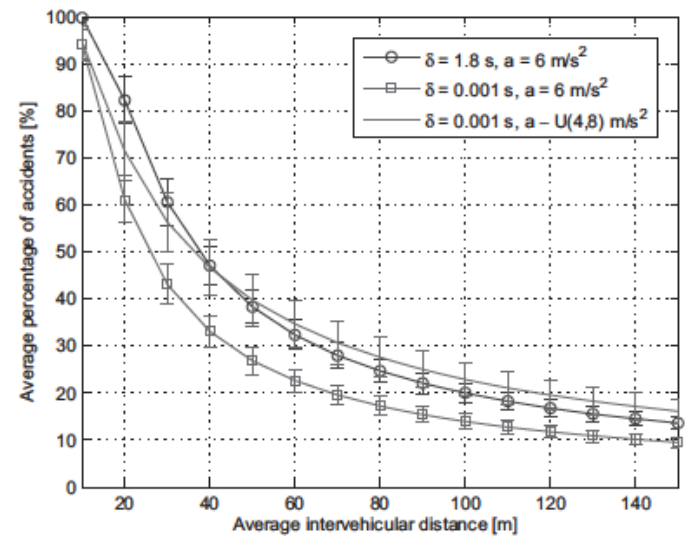

(a) Variability of deceleration with different notification delays

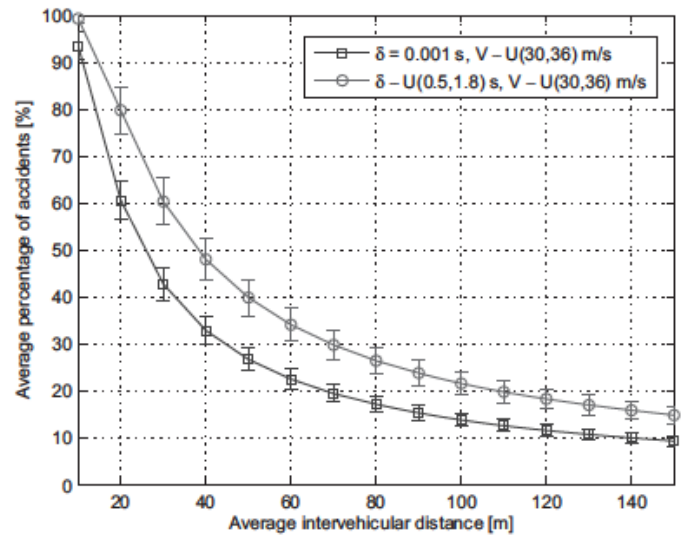

(b) Variability of velocity and notification delay

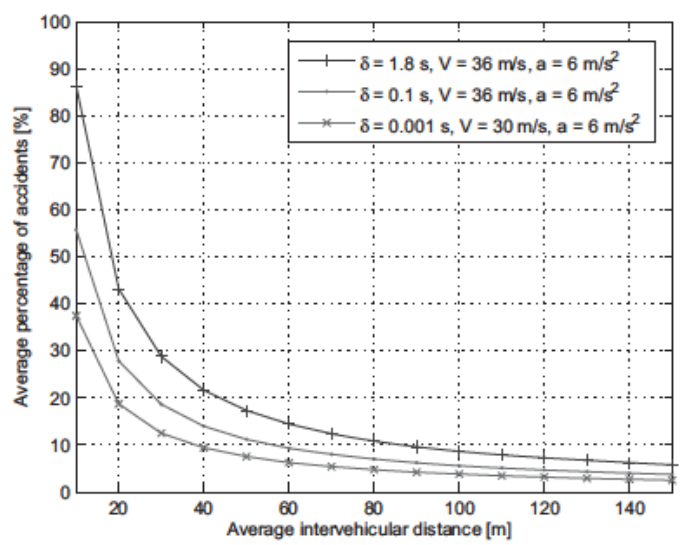

(c) Performance of the model with fixed parameters

Figure 3.17: Evaluation of the impact of the parameters' variability on the number of vehicle collisions 


\section{SIMULATION AND MATHEMATICAL VALIDATION OF A CCCA APPLICATION FOR VANETS USING IEEE 802.11P}

Fig. 3.17(a) shows the results when the velocities are randomly distributed. In this case if either deceleration or notification delay are kept constant it causes a reduction of the number of accidents. In fact, in this case it is noticeable the positive effect of a communication system able to deliver warning messages with short maximum delays and automatic vehicle response. Fig. 3.17(b) shows similar results when deceleration is kept constant at $a=6 \mathrm{~m} / \mathrm{s}^{2}$. The results however reveal that in general the variability of the kinetic parameters has a negative impact on the number of accidents. If the system is able to keep constant some of the parameters during the emergency event, an improvement can be achieved. The benefits of a warning collision system are even clearer in Fig. 3.17(c). When all the parameters remain constant, a shorter notification delay always results in fewer vehicle accidents.

Overall, these results suggest that a cooperative warning collision notification system combined with a vehicle control system able to smooth out the variations of speed and deceleration of the platoon of vehicles may improve the driver and passengers safety. In fact, more detailed conclusions can be extracted. As we said in the introduction, our model is useful to provide general guidelines about the design and operation of a CcCA application. For instance, a CcCA application may be based on a warning message delivered by an appropriate Human Machine Interface (HMI) (66), a specific sound for instance as in $(67)$, or by a fully automated braking response $(68,69)$. The latter is expected to provide better performance, but one may wonder if the former benefits from a communication system and for which range of parameters. Now, how can our model be applied to obtain relevant conclusions about these questions? First, it must be taken into account that for a reactive CcCA application, the only parameters in our model that can be controlled are delays, with the communication system, and decelerations, with some automated control response to the warning message. Let us recall that either the velocities of the vehicles and the intervehicular distance model the state of the traffic when the incident occurs and so both of them should be considered a random variable. Therefore, in the best case, the $\mathrm{CcCA}$ is able to provide a constant and short delay and enforce an appropriate constant deceleration. So, the curve in Fig. 3.17(b), provides the results for this case. If there is a warning message, but the driver still keeps the control of braking, a random reaction time should be added before the brake. This case is provided by the curve with uniform delay again in Fig. 3.17(c), assuming that the deceleration can be kept constant, which is not quite realistic. It is more reasonable 
that every driver applies also a different deceleration, which is the case shown in Fig. 3.16(b). But, as shown, in this case the actual delay is of little relevance. This has important implications in the design of the CcCA. The usual approach is to consider that the emergency messages must be sent as fast as possible $(14,51)$, but according to these results a higher delay could be traded off for other features such as reliability of warning message reception. For instance, adding a Request-To-Send/Clear-To-Send mechanism to avoid packet collisions due to hidden nodes (16). Or more importantly, the $\mathrm{CcCA}$ application should provide an acceleration control mechanism, so the margin in delay can be used to collect all the necessary information from neighbor vehicles to perform such control properly. This kind of insights on delay requirements is also important for designing $\mathrm{CcCA}$ applications based on predicting trajectory conflicts, in order to determine the time horizon for trajectory estimation (70).

However, if we consider a low speed and high density scenario, the delay has a remarkable influence. Fig. 3.18 shows the average percentage of accidents when velocities are uniformly distributed within 10 and $16 \mathrm{~m} / \mathrm{s}$. This scenario would model a urban road, where speed is relatively low but the vehicle density is high $^{1}$. And in this case, specially at short intervehicular distances corresponding to urban roads, the influence of delay is more noticeable, higher than that of deceleration. Therefore we can conclude that the use of an HMI message might not be sufficient to ensure safety and a special emphasis should be placed on providing automatic deceleration control. Moreover, in this scenario it is specially difficult for a communication system based on contention channel access (CSMA) to provide low delays, since the number of neighbors in range is high, unless additional congestion control mechanisms such as transmit power control are applied.

In fact, some of these conclusions can be drawn by directly examining eq. (3.12), that is, for high speeds it is more important to have good deceleration capabilities rather than to press the brake quickly, and conversely for low speeds. The previous discussion is provided to show that the model has potential to provide interesting qualitative and quantitative conclusions about the colliding process and the mechanisms that $\mathrm{CcCA}$ applications should include, but we have to remark that a precise definition of the

\footnotetext{
${ }^{1}$ Just for the sake of example, but let us remark that a log-normal distribution for intervehicular distances describes more accurately high density scenarios.
} 


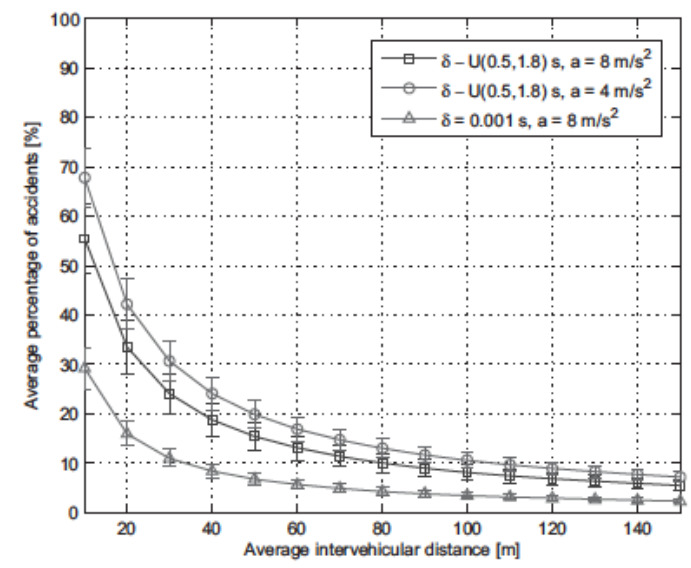

Figure 3.18: Average percentage of accidents in a low speed scenario with $V_{i} \sim U(10,16)$ $\mathrm{m} / \mathrm{s}$

scenarios of interest is still necessary, to set appropriate distributions and ranges for the parameters, which is left as future work.

Finally, as for the quantitative aspects of the results, the percentage of accidents might seem higher than expected, above $10 \%$ in many cases, as well as the slow decay of it for high intervehicular distances. This is first a consequence of the extreme case we are evaluating here, that is, the leading vehicle stops completely and immediately. It makes the collision of the first car of the platoon almost unavoidable in most of the cases. As a worst case approach, better outcomes are expected in reality. But also these results have to be interpreted with care, since using average intervehicular distances may lead to misleading conclusions. As an example, with the parameters used in Fig. $3.17(\mathrm{c}), V=36 \mathrm{~m} / \mathrm{s}, a=6 \mathrm{~m} / \mathrm{s}^{2}$ and $\delta=0.1 \mathrm{~s}$, the distance needed to stop is $111.6 \mathrm{~m}$. For an exponentially distributed intervehicular distance with mean $\bar{s}=60 \mathrm{~m}$, the probability of $s_{i}$ being less than $100 \mathrm{~m}$ is 0.81 , and even with a mean $\bar{s}=150 \mathrm{~m}$, this probability is still 0.48 . So the probability of collision is higher than one may intuitively think, specially for the first vehicles in the chain. Therefore, even at relatively high intervehicular distances, the collisions are mainly suffered by the first and second vehicle, which accounts for the $10 \%$ of accidents for our example with $N=20$ vehicles. 


\subsection{Final remarks}

The use of CcCA capabilities in vehicular networks guarantees passengers to drive with a much higher level of safety (when full CcCA support is implemented in all vehicles within the platoon). The dependence of collided vehicles against intervehicular distance actually relies upon the speed of vehicles, the reaction time to the event, the transmission power (only for $\mathrm{CcCA}$ ) and to a remarkable extent the percentage of CcCA technology penetration among vehicles inside the platoon. Reaction time can increase the number of accidents if the brake system relies on the driver's reaction when compared with the automatic brake system of the vehicle, mostly for middle intervehicular distances. Speed always plays a crucial role in results. Obviously, the higher the speed of vehicles, the greater the number of accidents. Transmission power is also a critical factor when we compare simulations of CcCA applications for different values of this parameter. As we could see here, a very simple one-hop delivery policy can perform well when power can reach most of the vehicles in the convoy. However, when it is not possible, the number of accidents increase partially, as illustrated in previous scenarios. On the other hand, the transition between the current state of vehicular traffic (without communication capabilities) and that with full $100 \%$ support must be seriously evaluated. We have shown that for CcCA applications, when not all vehicles support communication capabilities some undesiderable phenomena may appear, turning the system perform worse and implying a larger amount of accidents for some cases in which not using CcCA would be a better idea.

Along with simulations of CcCA scenarios presented in this chapter, we have proposed a stochastic model for the calculation of the probabilities of collision in a chain of vehicles where a warning collision system is in operation. The fact that a warning notification system is used allows us to overcome the difficulties for obtaining stochastic models for such vehicular scenarios, since we can assume that all the drivers/vehicles react to the warning message independently, and therefore the motion equations can be simplified. We also propose a good matching approximation to the exact model to further reduce the required computations to calculate the vehicle collision probabilities. In both cases, the validation of theoretical results has been carried out by simulation in NCTUns 6.0. Communications in the model were abstracted and characterized by an appropriate message notification delay including communication latency and driver 


\section{SIMULATION AND MATHEMATICAL VALIDATION OF A CCCA APPLICATION FOR VANETS USING IEEE 802.11P}

reaction times, giving support to the possibility of evaluating different technologies. Similarly, different probability distributions for the intervehicular spacing can be incorporated seamlessly into the model, due to the fact that the distribution of the initial intervehicle spacing is independent of the actions that drivers make after receiving the warning messages. Here we have used an exponential distribution, which is considered appropriate for low vehicle traffic densities, but using log-normal distributions is better justified under conditions of high vehicular density. Eventually, we compute the probability that collisions occur in different forms (both vehicles in motion, one stopped and one in motion, etc.), which constitutes a promising way to define detailed accident severity functions, that is, by assigning different degrees of severity to each collision possibility.

In the next chapter we will continue by designing and evaluating three different relay schemes to complement results obtained so far, and analyze under which circumstances one-hop communications are enough for an acceptable performance of the developed CcCA application.

\section{Nomenclature}

$N \quad$ Number of total vehicles in the chain (excluding the leader, which collides first).

$C_{i} \quad$ Vehicle $i \in\{0, . ., N\}$ in the chain.

$a_{i} \quad$ Braking deceleration of vehicle $C_{i}$.

$L \quad$ Length of vehicles (constant for all $C_{i}, i \in\{0, . ., N\}$ ).

$s_{i} \quad$ Intervehicular distance between the front bumpers of $C_{i}$ and $C_{i-1}$.

$V_{i} \quad$ Initial speed of vehicle $C_{i}$.

$S_{j, k} \quad$ State of the Model's Markov chain representing the situation of $j$ vehicles collided, and $k$ vehicles stopped successfully.

$p_{i} \quad$ Collision probability of vehicle $C_{i}$.

$\mathbf{P}^{N} \quad$ Matrix of probabilities of going from the initial state to each of the $N+1$ final states in $N$ steps.

$\Pi_{i} \quad$ Probability of reaching the final outcome with $i$ collided vehicles.

$N_{a c c} \quad$ Mean of the total number of accidents in the chain.

$t_{0} \quad$ Time instant in which the leader of the chain collides. 
$l_{i} \quad$ Total distance traveled by vehicle $C_{i}$ since the emergency event occurs at time $t_{0}$.

$T_{m, i} \quad$ Message reception delay.

$T_{r, i} \quad$ Reaction time to the reception (either for automatic as for human assisted driving).

$\delta_{i} \quad$ End-to-end delay of the transmitted notification packet between $C_{0}$ and $C_{i}, \delta_{i}=T_{m, i}+T_{r, i}$.

$d_{s, i} \quad$ Distance taken by vehicle $C_{i}$ to stop completely without colliding.

$d_{c, i} \quad$ Average distance traveled by the vehicle $C_{i}$ in case of collision.

$\lambda \quad$ Design parameter (vehicular density) for the exponential distribution of the intervehicular distance $s_{i}$.

$\overline{l_{i}} \quad$ Average distance traveled by $C_{i}$.

$t_{c_{1}, i}(x) \quad$ Time instant at which collision type (1) has occurred.

$t_{c_{2}, i}(x) \quad$ Time instant at which collision type (2) has occurred.

$t_{c_{3}, i}(x) \quad$ Time instant at which collision type (3) has occurred.

$i n f_{j} \quad$ Lower integration limit.

sup $_{j} \quad$ Upper integration limit.

$q_{c_{j}, i} \quad$ Probability of the different ways in which vehicle collisions may occur. 
3. SIMULATION AND MATHEMATICAL VALIDATION OF A CCCA APPLICATION FOR VANETS USING IEEE 802.11P 


\section{Testing viability of relay policies for reactive CcCA applications in VANETs}

\subsection{Introduction and motivation}

It was obvious from Chapter 3 that under certain circumstances, one-hop communications in $\mathrm{CcCA}$ applications can suffice when the number of vehicles belonging to the platoon is not too high, or when the transmission power is high enough to improve the reception ratio of receiving nodes. One-hop communication schemes do not presumably fill the channel with unnecessary relaying packets that might incur into multiple channel collisions, thus its initial attractiveness. However, we could notice that for relatively long chains (more than 20 vehicles), and even more remarkable for low transmission power values, the percentage of accidents in a platoon would not be minimized because vehicles located in the middle of the chain would neither receive the message timely to start reaction, nor would they have enough time to react to front vehicles' sudden braking actions. Under these particular circumstances, it could be interesting to analyze how relaying ${ }^{1}$ can solve this issue and reduce the height of the elbow of the central region in the graphs of the percentage of accidents in the platoon (see, for example, Figs. 3.3 and 3.7). Relaying can, whatsoever, increase the ratio of collided packets sent to the channel, since the number of packets delivered is much higher that with simple one-hop retransmissions. The percentage of accidents by using relaying

\footnotetext{
${ }^{1}$ By relaying we mean here the retransmission of a packet by a vehicle that was not the original sender.
} 


\section{TESTING VIABILITY OF RELAY POLICIES FOR REACTIVE CCCA APPLICATIONS IN VANETS}

schemes, as well as the evaluation of the channel performance when these schemes are employed is the main object of evaluation in this Chapter.

In summary, the main goal of this Chapter is to evaluate if warning notification packet relaying can practically improve safety-related applications and when such mechanisms should be used to reach the necessary number of vehicles affected by a risky situation in a platoon, that is, when they cannot be informed with only a one-hop transmission. Therefore, determining which vehicles could be involved in an accident according to their situation in the platoon is necessary to assess if relaying is worth enough. The relay policies we evaluate are the schemes proposed in (17) (NB and IBIAS), with some improvements. These schemes (Algorithms 2 and 3) will be compared together with a scheme without relaying (Algorithm 1), actually, the same scheme as the one evaluated in Chapter 3. We perform a thorough evaluation by simulation, testing the behavior of $\mathrm{CcCA}$ relaying according to different parameters that have direct influence like the transmission power, background traffic and percentage of vehicles supporting CcCA. This latter case is specially interesting since the adoption of vehicular technologies will be gradual. We also provide different metrics that help showing the real utility of the mechanism.

The rest of this Chapter is organized as follows. In Section 4.2 a description of relevant related work is introduced, focusing exclusively on relaying schemes that might be useful for the purpose of evaluation in this Chapter. In Section 4.3 we describe in detail the most important issues concerning the different simulation cases we studied here and we evaluate the performance of the three relay policies under different configuration parameters. In this section, some illustrative results are also shown and discussed. Section 4.4 finishes Chapter 4 with some concluding remarks, extracting the most important aspects of the use of relaying for $\mathrm{CcCA}$ applications.

\subsection{Related Work}

Several studies on the reduction and minimization of channel occupation and end-toend delay in CcCA applications have been conducted according to different perspectives $(17,71,72)$. Biswas et al. (17) proposed two protocols for broadcasting CcCA-related safety messages, NB (Naïve Broadcast) and I-BIA (Intelligent Broadcast with Implicit Acknowledgement). In both mechanisms, when a vehicle receives a warning message 


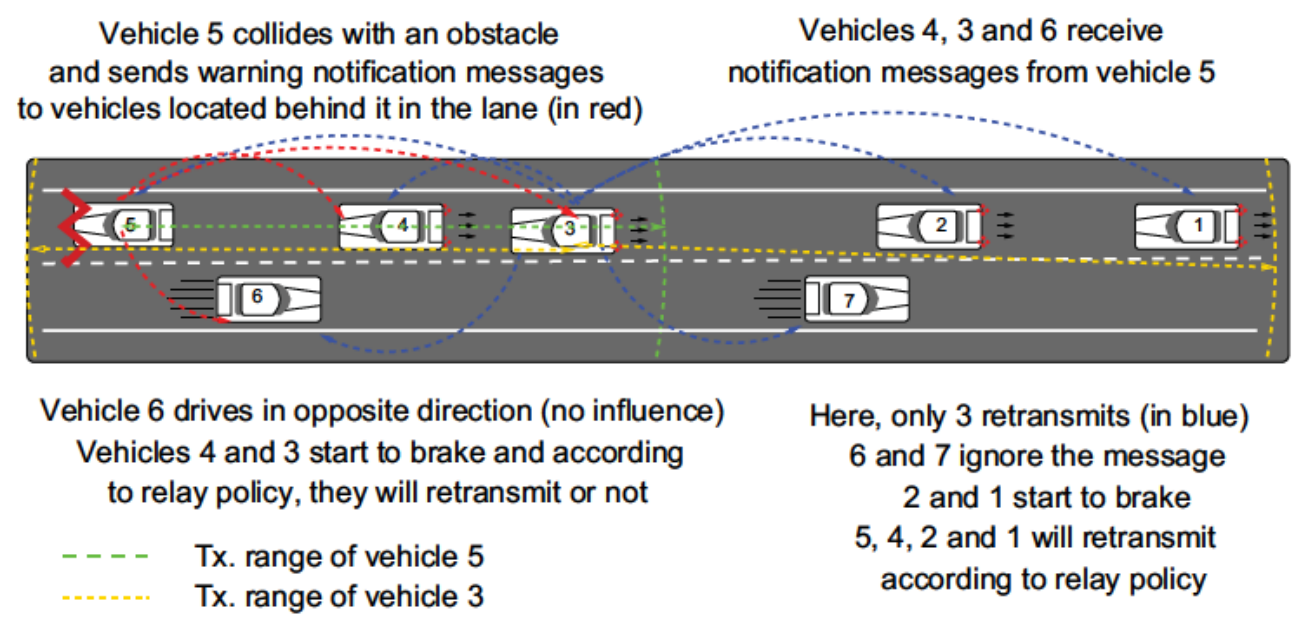

Figure 4.1: General scenario for testing relay policies in VANETs with 802.11p communication capabilities

coming from other vehicle located ahead, a retransmission will follow. In I-BIA, however, messages will be only retransmitted after a random period in which a receiver does not hear the retransmission of a further vehicle which previously received the same message. I-BIA notably reduces the number of data packets sent to the channel (when compared with NB), but random times are not appropriate to give a complete view of the optimum time which vehicles should wait to retransmit (i.e. to maximize the delivery rate and on the other hand to minimize the end-to-end delay).

In (71) a power control procedure is used to avoid redundant use of the channel bandwidth by an estimation of the transmission power according to the safety distance. A remarkable reduction in the number of car accidents is obtained (as well as a smaller packet delivery delay). Power control can be a good mechanism when the scenario is not highly dynamic, but in the case fading, attenuation and scattering affect continuously the reception process power control can be quite complex to implement. Furthermore, in (71) authors use the deterministic Two Ray propagation model (73) which does not capture realistically the behavior of electromagnetic propagation in vehicular environments (51). In (72), VeSOMAC, a self-configuring TDMA protocol for the transmission of CWM (Collision Warning Messages) is described. Access to the medium is distributed into slots and when compared with contention based protocols like that of the WAVE architecture, it is shown that it is possible to obtain deterministic and lower delay bounds than those of IEEE 802.11p based devices (49). We will 


\section{TESTING VIABILITY OF RELAY POLICIES FOR REACTIVE CCCA APPLICATIONS IN VANETS}

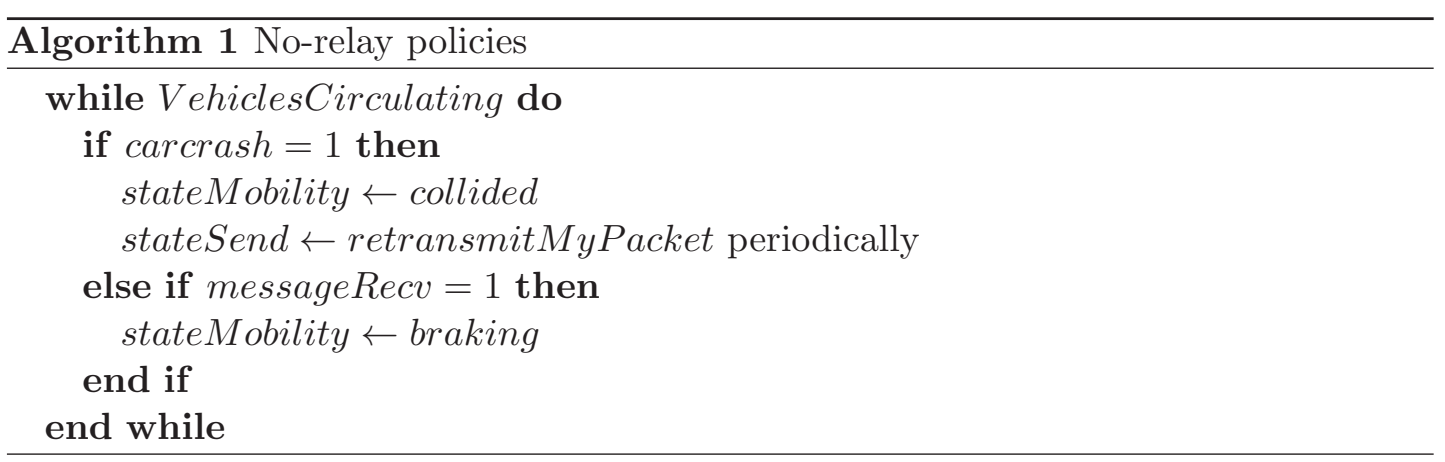

show nevertheless that, despite this fact, IEEE 802.11 p can perform well for CcCA applications. In this work, we focus on determining which is the situation that makes relaying useful rather than providing a particular algorithm, that is, when relaying really improves the functionality of the system when compared to a single transmission. Since there is a high number of parameters that have influence on the effectiveness of $\mathrm{CcCA}$, we provide a thorough evaluation by using different metrics that help deciding if collisions are actually avoided because of the reception of a warning message.

Some research has been also devoted to carry out realistic testbeds in which $\mathrm{CcCA}$ has been evaluated. It is the case of (74), in which different algorithms are presented to guarantee collision avoidance in highway mergings and traffic circles, by using techniques related to partial order and order preserving dynamics. They show the real time applicability of such system by evaluating the behavior of two vehicles in a roundabout scaled drill in which cars are running continuously.

\subsection{Evaluation and results}

As in the previous Chapter, we use here the simulation platform NCTUns 6.0 Network Simulator, see Appendix A, to test the viability of using relay mechanisms for CcCA applications.

\subsubsection{General scenario description}

Our evaluation study aims at assessing the usefulness of relaying warning messages in CcCA applications in scenarios where a collision makes it necessary for a platoon of vehicles to try to stop in the shortest time possible and avoid crashing against preceding vehicles. The basic scenario is thus based on a two way road in which vehicles drive 


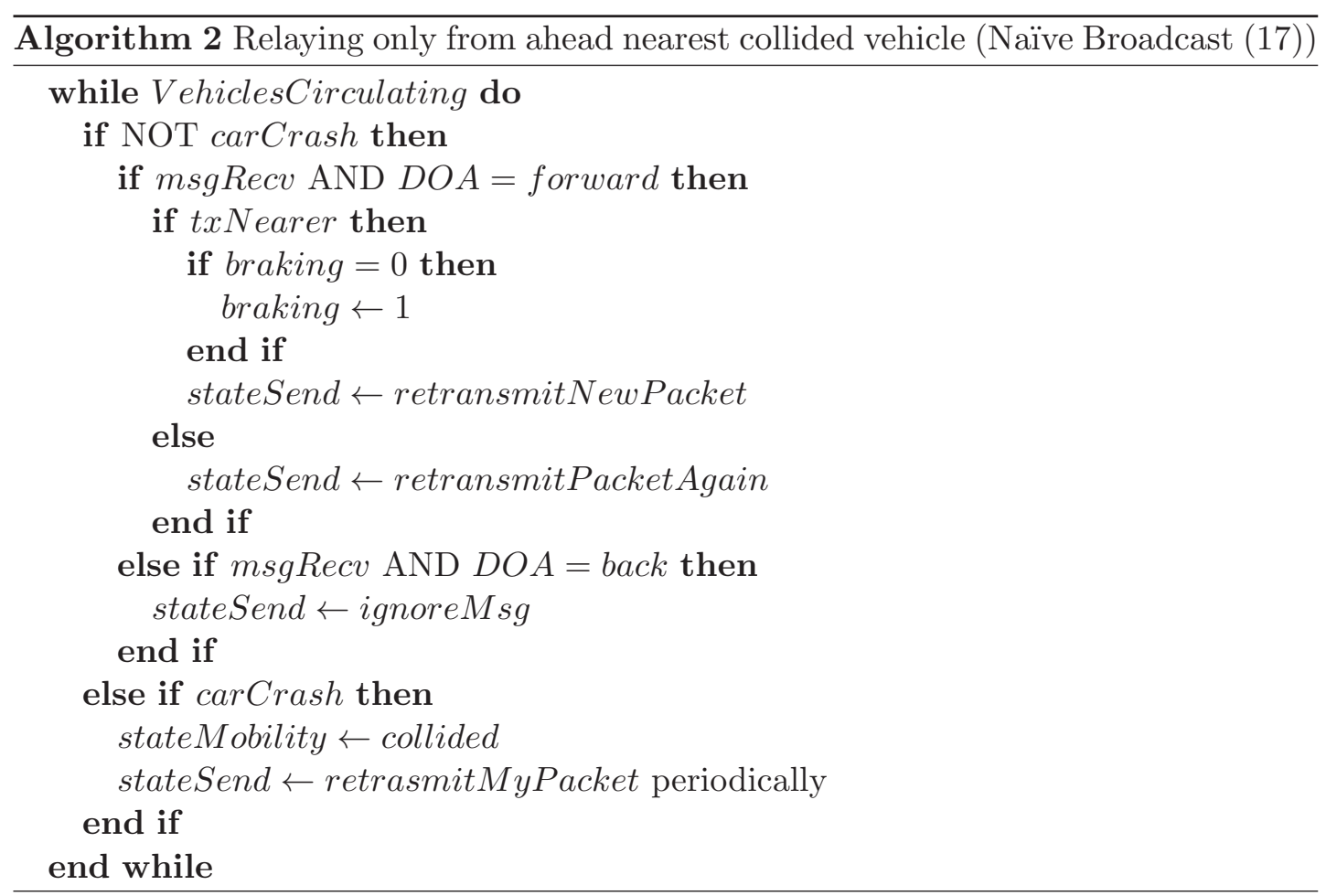

in opposite directions, where only one of them is taken into consideration (Fig. 4.1). Vehicles drive in convoy, reacting to the first collision of another car according to two possible schemes: starting to brake because of a previously received warning message transmitted by a collided vehicle (directly from the source or relayed) or starting to decelerate after noticing a reduction in the speed of the vehicle immediately ahead (the relay Algorithms are explained later in detail).

Vehicles will not be able to change their direction of movement (worst case situation). For all simulation cases speed is set to $33 \mathrm{~m} / \mathrm{s}$ (around $74.5 \mathrm{mph}$ ), a value which is used in average by vehicles driving on highways (75). For those cases in which we analyze the influence of the intervehicular distance on the metrics under consideration, this parameter ranges between 6 and $72 \mathrm{~m}$. Usefulness of relaying is tested by using two different values for the transmission power, $28 \mathrm{dBm}$ and $10 \mathrm{dBm}$ respectively (the first value is an upper bound as presented in the draft standard IEEE 802.11p (48) and the second one is low enough as to evaluate in detail how relaying performs). When noticing a crash, each vehicle will transmit (according to its respective relaying algorithm) at a maximum rate of 1 packet every $20 \mathrm{~ms}$. Vehicles will begin to brake after an interval 


\section{TESTING VIABILITY OF RELAY POLICIES FOR REACTIVE CCCA APPLICATIONS IN VANETS}

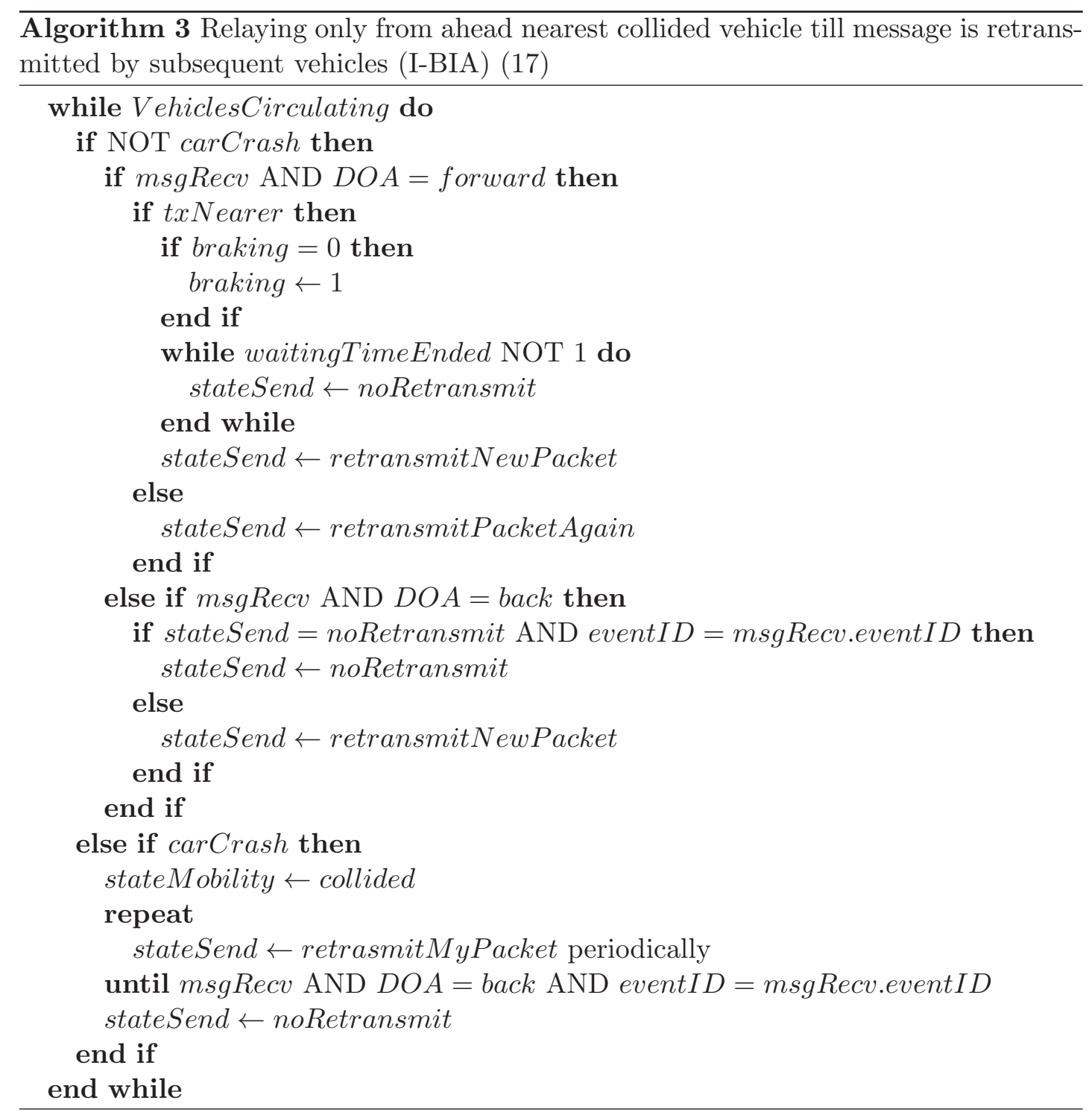

of time consisting of the transmission time plus the reaction time of the driver $(0.2 \mathrm{~s}$ plus a uniformly distributed interval of 0.5-1 s). Relay mechanisms are also assessed subject to different penetration ratios of the CcCA technology in the market. Background data traffic is additionally considered in order to determine the functionality of the relay procedures when influenced by different loads of the communications channel.

The study is conducted according to different performance metrics which are described next:

- Percentage of accidents. This metric shows directly the improvement of the 
system when using CcCA under different relaying (or non-relaying) schemes. In this case, for a fixed driving speed, the percentage of collided vehicles is represented against different values of the average intervehicular distance parameter, in Simulation case 1 (Fig. 4.2). In Simulation case 2 the percentage of collided vehicles is shown against the technology penetration ratio (Fig. 4.5). Finally, in Simulation case 3 this parameter is faced against the background data traffic present in the channel, in addition to the safety related information (Fig. 4.6).

- Successful Delivery Ratio (SDR). This performance measure is used to quantify the effectiveness of informing vehicles over an incidence of collision. We measure the notification warning delivery success for the different Algorithms we assess. Values are averaged for the whole platoon in Tables 4.2 and 4.3.

- Merit factor in reception. This metric represents the usefulness of the delivery of warning notification messages. It is calculated by the Eq. $M F=(1-P) \times \mathrm{SDR}$, where $M F$ denotes the Merit factor in reception (1 highest usefulness of messages, 0 lowest usefulness of messages), $P$ is the percentage of accidents in the platoon and SDR is the Successful Delivery Ratio. This metric is represented according to the position of the vehicle in the platoon and the average intervehicular distance (Fig. 4.3).

- Average end-to-end delay. Here we measure the time taken by a vehicle to receive a warning notification message since the message was first sent, that is, by the source vehicle who registered the incidence. It is calculated to quantify the delay in transmitting safety-related information to vehicles. This metric is represented according to the position of the vehicle in the platoon and the average intervehicular distance (Fig. 4.4).

- Packet Collisions Ratio (PCR). This metric is used to calculate the ratio of collided packets according to the relay algorithm and the transmission power used. Values are shown in Tables 4.2 and 4.3.

The three different relay policies evaluated in this study (we have chosen these schemes because they cover the three basic ways of relaying broadcast messages in the application layer (17)) are presented next: 


\section{TESTING VIABILITY OF RELAY POLICIES FOR REACTIVE CCCA APPLICATIONS IN VANETS}

\subsubsection{Algorithm 1}

There is no relay mechanism. Vehicles transmit warning notifications periodically only when they crash. This scheme is exactly the same as the one used for the simulations in Chapter 3.

\subsubsection{Algorithm 2}

Vehicles will only retransmit messages coming from cars located ahead (forward DOA, direction of arrival). However, if a message is received by a car from a preceding vehicle which is closer to it than the first sender of the message being retransmitted till now, the new message will replace the previous one. If the vehicle collides, from then onward the vehicle will only retransmit messages with his own eventID (identification number of incidence).

\subsubsection{Algorithm 3}

Vehicles will retransmit messages from preceding vehicles only after a random waiting time interval during which they do not hear the retransmission of another vehicle located behind. If during this time interval a vehicle receives from a following vehicle the message it was going to retransmit, then the retransmission will be canceled (it is supposed that the packet is already relayed).

\subsubsection{Results}

In this subsection the results obtained for three different simulation cases are presented and discussed. Simulations are performed according to a $99 \%$ confidence interval for all the statistics we measure ( $t$-Student distribution) and the Nakagami fading model is used (51). Speed is kept fixed for $33 \mathrm{~m} / \mathrm{s}$ as well as the number of vehicles in the platoon: 31 cars. Reaction to stimuli is based on the natural human delay to detect the incidence and start braking ( $0.2 \mathrm{~s}$ plus a uniformly distributed value in the time interval [0.5-1] s). Intervehicular distance ranges between 6 and 72 meters, in 3-meter steps (where car position is shifted according to a normal distribution with respect to the average value of the position on the road (meters) and standard deviation proportional to the intervehicular distance too). Please, refer to Table 4.1 for further details on configuration values for simulation parameters. 
Table 4.1: Configuration parameters for the simulation cases

\begin{tabular}{cccccc}
\hline $\begin{array}{c}\text { Simulation } \\
\text { case }\end{array}$ & Tx. power & $\begin{array}{c}\text { Relay } \\
\text { algorithm }\end{array}$ & Interv. dist. & $\begin{array}{c}\text { \% Tech. } \\
\text { penetration }\end{array}$ & $\begin{array}{c}\text { Background } \\
\text { data traffic }\end{array}$ \\
\hline 1 & $10,28 \mathrm{dBm}$ & $1,2,3$ & {$[6-70] \mathrm{m}$} & $100 \%$ & $\mathrm{~Kb} /\left(\mathrm{s}^{*}\right.$ vehicle $)$ \\
\hline 2 & $10,28 \mathrm{dBm}$ & $1,2,3$ & $25,60 \mathrm{~m}$ & {$[0-100] \%$} & $\mathrm{~Kb} /\left(\mathrm{s}^{*}\right.$ vehicle $)$ \\
\hline 3 & $10,28 \mathrm{dBm}$ & $1,2,3$ & $25,60 \mathrm{~m}$ & $100 \%$ & $\begin{array}{c}{[0-700]} \\
\mathrm{Kb} /\left(\mathrm{s}^{*} \text { vehicle }\right)\end{array}$ \\
\hline
\end{tabular}

\subsubsection{Simulation case 1. Influence of transmission power}

In this first simulation we evaluate the basic differences between using each of the aforementioned Algorithms for relaying with respect to different values of the transmission power. First of all, if we observe the Graphs of Fig. 4.2 we can notice that, despite the differences in the functionality of Algorithms 2 and 3, when the transmission power is enough to cover all vehicles in the platoon (in this case $28 \mathrm{dBm}$ is enough, IEEE 802.11p standard (48)), relay behaves like the case without relaying capabilities (Graph 4.2.b). However, the importance of relay arises only when transmission power is not enough to reach an advisable number of vehicles (Graph 4.2.a).

On the other hand, as an additional important goal, every relaying algorithm for CcCA applications must always try to minimize the channel occupation when necessary, because it might be shared with other critical safety applications which could also require a certain bandwidth. Graphs in Fig. 4.3 show the evolution of the Merit factor in reception for the different average intervehicular distances and the position of vehicles in the platoon, according to the transmission power and the relay Algorithm used. As can be seen, the worst case corresponds to that of Graph 4.3.a, where only one hop transmissions of $10 \mathrm{dBm}$ (low transmission power) are not heard by an acceptable number of vehicles in the platoon. This causes the Merit factor in reception to reach its lowest values on the left (because vehicles collide massively) and on the right (because vehicles at these average intervehicular distances are too far to receive notification packets). In 4.3.b it is easy to see that the Merit factor in reception evolves in the same way like in Algorithms 2 and 3. The other four Graphs in Fig. 4.3, namely 4.3.c, 4.3.d, 4.3.e and 4.3.f, show a similar behavior, where SDR is high enough to reduce notably the number of accidents. 


\section{TESTING VIABILITY OF RELAY POLICIES FOR REACTIVE CCCA APPLICATIONS IN VANETS}

a) Transmission power $10 \mathrm{dBm}$

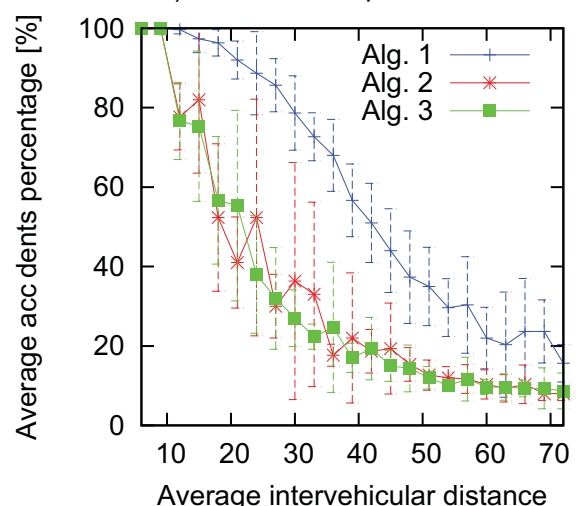

b) Transmission power $28 \mathrm{dBm}$

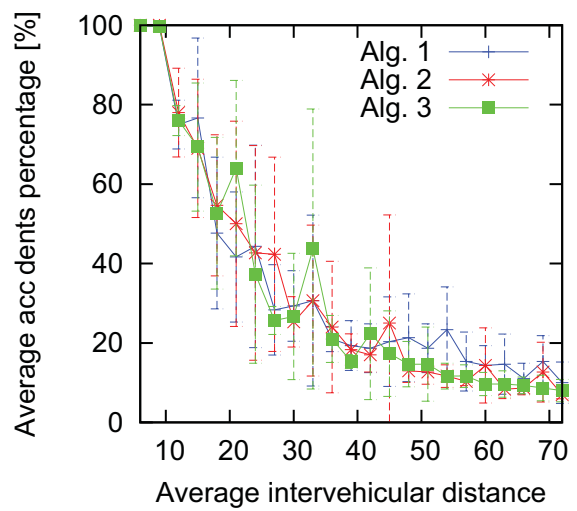

Figure 4.2: Percentage of accidents as a function of the average intervehicular distance

a) $10 \mathrm{dBm}$, Algorithm 1

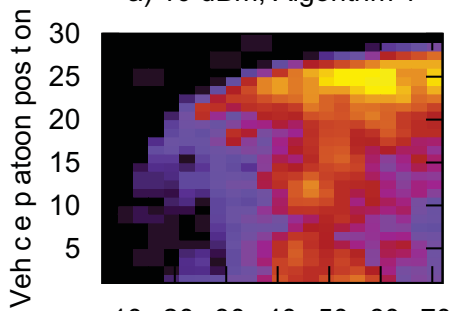

$\begin{array}{lllllllllllllllllllll}10 & 20 & 30 & 40 & 50 & 60 & 70 & 10 & 20 & 30 & 40 & 50 & 60 & 70 & 10 & 20 & 30 & 40 & 50 & 60 & 70\end{array}$ Average intervehicular distance $[\mathrm{m}]$

d) $28 \mathrm{dBm}$, Algorithm 1

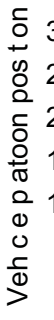

b) $10 \mathrm{dBm}$, Algorithm 2

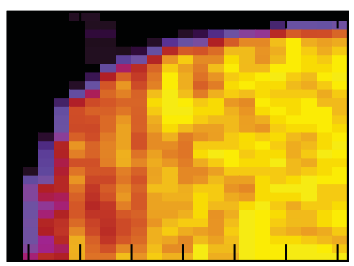

e) $28 \mathrm{dBm}$, Algorithm 2

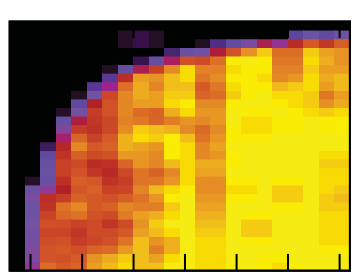

f) $28 \mathrm{dBm}$, Algorithm 3

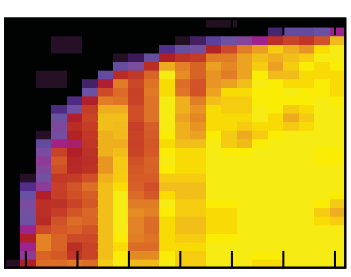

$\begin{array}{lllllllllllllllllllll}10 & 20 & 30 & 40 & 50 & 60 & 70 & 10 & 20 & 30 & 40 & 50 & 60 & 70 & 10 & 20 & 30 & 40 & 50 & 60 & 70\end{array}$

Average intervehicular distance $[\mathrm{m}]$

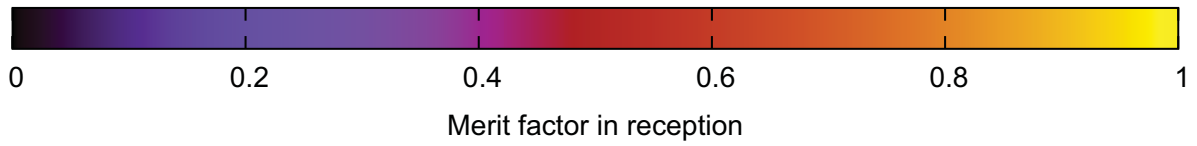

Figure 4.3: Merit factor in reception for the system of vehicles

In addition, when comparing Algorithms 2 and 3 after looking at Tables 4.2 and 4.3 we find out that Algorithm 3 reduces significantly the number of packets sent to the 
Table 4.2: Successful Delivery and Packet Collisions Ratio (SDR, PCR), tx. power 10 $\mathrm{dBm}$

\begin{tabular}{cccc}
\hline Power 10 dBm & Algorithm 1 & Algorithm 2 & Algorithm 3 \\
\hline SDR & $87.62 \%$ & $91.92 \%$ & $94.23 \%$ \\
\hline PCR & $23.19 \%$ & $55.16 \%$ & $25.75 \%$ \\
\hline Utility factor & 0.4810 & 0.8188 & 0.8467 \\
\hline
\end{tabular}

Table 4.3: Successful Delivery and Packet Collisions Ratio (SDR, PCR), tx. power 28 $\mathrm{dBm}$

\begin{tabular}{cccc}
\hline Power 28 dBm & Algorithm 1 & Algorithm 2 & Algorithm 3 \\
\hline SDR & $95.55 \%$ & $94.64 \%$ & $95.81 \%$ \\
\hline PCR & $11.82 \%$ & $58.09 \%$ & $28.78 \%$ \\
\hline Utility factor & 0.8297 & 0.8486 & 0.8556 \\
\hline
\end{tabular}

medium (deduced by the lower amount of packet collisions, PCR). The SDR is thus higher for those cases in which Algorithm 3 is used. As can be also inferred from this Table (comparing Algorithms 2 and 3), using higher values for the transmission power turns out in an increase of the Merit factor in reception, since the number of packet collisions is also smaller.

To finish with this first simulation case, an evaluation of the end-to-end delay evolution is also carried out. SDR is important, but it is also critical to deliver messages as soon as possible (mainly because their usefulness depends on the celerity taken to deliver them). As we can observe in Fig. 4.4, Graphs 4.4.a and 4.4.d correspond to the case without relay capabilities. For short intervehicular distances, transmission power is enough to reach all vehicles in the platoon, but for high distances, even vehicles close to the first collided car register a high end-to-end delay (Graph 4.4.a, further vehicles experience a lower delay because the message they receive belongs to a car behind the first one, which obviously collides later). In Graph 4.4.d, however, transmission power is enough to reach the whole platoon, and this implies that in general the end-to-end delay is also lower. On the other hand, when relaying is used, a higher number of packets must be sent to the channel. For low values of the transmission power in Algorithm 2 , the higher number of packet collisions implies higher delays (that is surprisingly minimized by Algorithm 3 due to its lower occupation of the medium, Graphs 4.4.b and 4.4.c). Using $28 \mathrm{dBm}$ of transmission power results in general in a shorter end-to-end delay (Graphs 4.4.e and 4.4.f). 


\section{TESTING VIABILITY OF RELAY POLICIES FOR REACTIVE CCCA APPLICATIONS IN VANETS}

a) $10 \mathrm{dBm}$, Algorithm 1

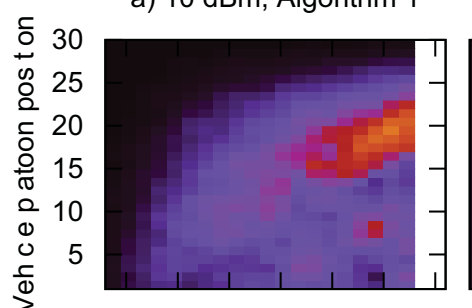

$\begin{array}{lllllllllllllllllllll}10 & 20 & 30 & 40 & 50 & 60 & 70 & 10 & 20 & 30 & 40 & 50 & 60 & 70 & 10 & 20 & 30 & 40 & 50 & 60 & 70\end{array}$ Average intervehicular distance [m]

d) $28 \mathrm{dBm}$, Algorithm 1

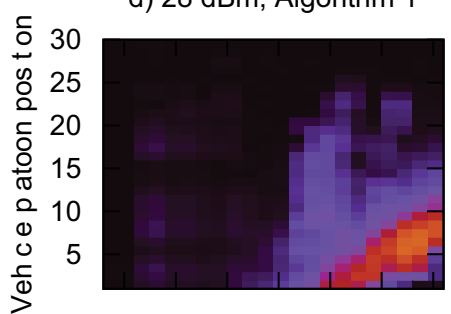

$\begin{array}{lllllllllllllllllllll}10 & 20 & 30 & 40 & 50 & 60 & 70 & 10 & 20 & 30 & 40 & 50 & 60 & 70 & 10 & 20 & 30 & 40 & 50 & 60 & 70\end{array}$ Average intervehicular distance $[\mathrm{m}]$

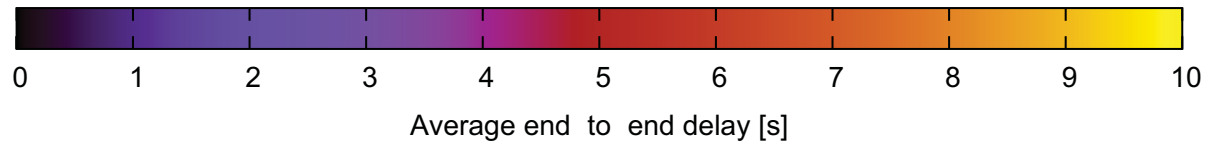

Figure 4.4: Average accident notification delay

From these results we can conclude that relaying is only necessary when the transmission power is not enough to cover a large amount of vehicles in danger of collision. Furthermore, it also needs more bandwidth to retransmit messages to the channel when compared with no-relay (Algorithm 1), whereas no-relay policies do not in general occupy the medium that much and offer at the same time a lower end-to-end delay when high enough transmission power values are used.

\subsubsection{Simulation case 2. Influence of percentage of CcCA technology penetration}

In this second simulation test we want to analyze thoroughly how relay mechanisms can perform under different penetration stages of the technology in the market.

As we can see from the Graphs in Fig. 4.5, the trend of the relay Algorithms 2 and 3 is the same regardless of the transmission power used by vehicles. For these 
a) $10 \mathrm{dBm}$, Intervehicular distance $25 \mathrm{~m}$

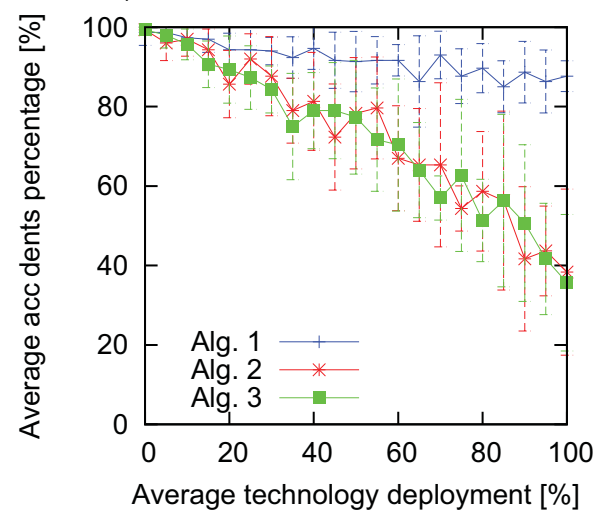

c) $28 \mathrm{dBm}$, Intervehicular distance $25 \mathrm{~m}$

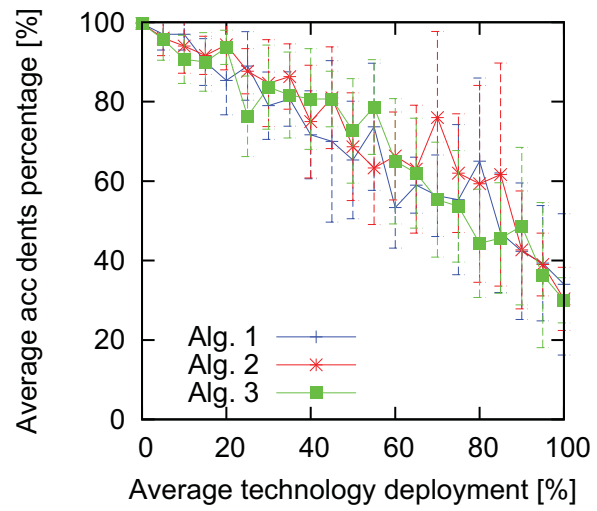

b) $10 \mathrm{dBm}$, Intervehicular distance $60 \mathrm{~m}$

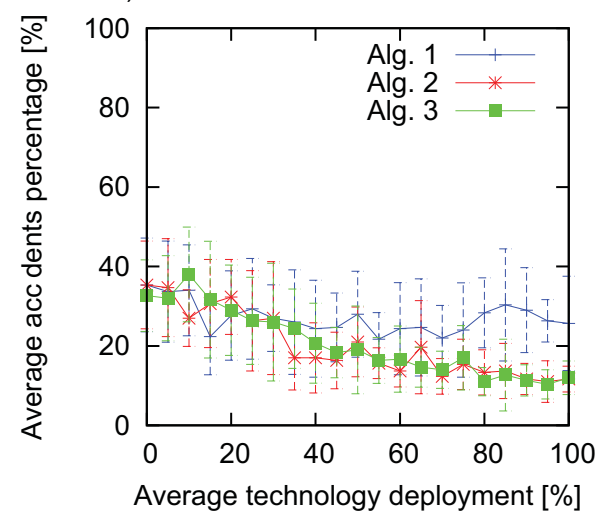

d) $28 \mathrm{dBm}$, Intervehicular distance $60 \mathrm{~m}$

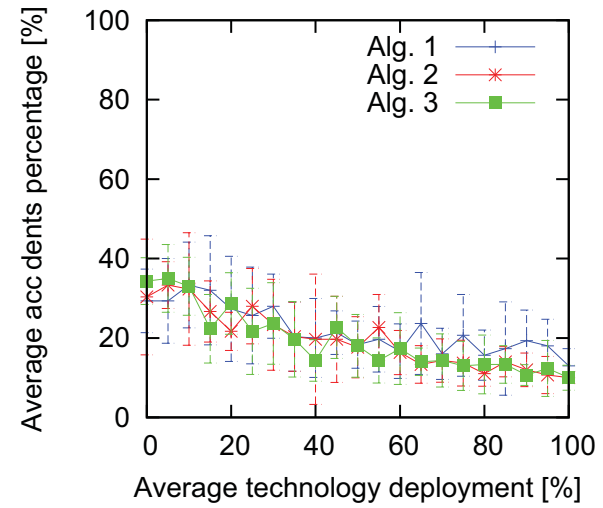

Figure 4.5: Percentage of accidents as a function of the average percentage of technology penetration in the market

Algorithms the percentage of collided vehicles is always reduced with an increase of the technology penetration percentage. However, curves are steeper for low intervehicular distances than for higher values of this parameter, that is to say, as the penetration of the technology in the market increases, the improvement on passengers' safety is more noticeable for low intervehicular distances. Nevertheless, the channel occupation of Algorithms 2 and 3 is much higher than that for Algorithm 1 (no relay). This can be stringent for other safety applications which may need to use the channel too. As regards Algorithm 1, lower values of transmission power lead to higher ratios of collided vehicles (Graphs 4.5.a and 4.5.b). When increasing transmission power for Algorithm 1 , it behaves very similarly to relaying mechanisms (Algorithms 2 and 3). 


\section{TESTING VIABILITY OF RELAY POLICIES FOR REACTIVE CCCA APPLICATIONS IN VANETS}

Table 4.4: Average accidents percentage for general background data traffic

\begin{tabular}{cccc}
\hline $\begin{array}{c}\text { Tx. power, Av. } \\
\text { distance }\end{array}$ & Algorithm 1 & Algorithm 2 & Algorithm 3 \\
\hline $10 \mathrm{dBm}, 25 \mathrm{~m}$ & $94.72 \%$ & $50.18 \%$ & $54.97 \%$ \\
\hline $10 \mathrm{dBm}, 60 \mathrm{~m}$ & $29.81 \%$ & $12.63 \%$ & $12.21 \%$ \\
\hline $28 \mathrm{dBm}, 25 \mathrm{~m}$ & $77.84 \%$ & $63.69 \%$ & $62.84 \%$ \\
\hline $28 \mathrm{dBm}, 60 \mathrm{~m}$ & $24.33 \%$ & $14.69 \%$ & $13.21 \%$ \\
\hline
\end{tabular}

From these results we can thus conclude that it is necessary to evaluate under which circumstances relay is critical, because it implies a higher occupation of the channel. Furthermore it is proved that using non-relay mechanisms with standard values of the transmission power $(28 \mathrm{dBm},(48))$ can be sufficient to reach a reasonable number of vehicles in the platoon and obtain a behavior very similar to what could be achieved with relaying, and at the same time have a much lower occupation of the medium. Moreover, during the early stages of $\mathrm{CcCA}$ deployment relaying cannot guarantee that a message will be retransmitted to those vehicles in a platoon. Using higher values for the transmission power and employing derivatives of Algorithm 1 can be enough and even more efficient to deliver safety related information.

\subsubsection{Simulation case 3. Influence of background traffic}

In this case we test how background data traffic in the channel can affect the functionality of the CcCA notification technology according to the transmission power used and the particular relaying Algorithm employed.

From Graphs in Fig. 4.6 it is easy to infer that the higher rate of background data traffic produced by vehicles, the higher the number of car accidents in the platoon (regardless of the relay algorithm used). However, Algorithm 1 (no relay) seems to be more affected by the influence of background data traffic, as can be deduced from the smaller number of warning notification messages that are sent to the radio channel in comparison to the other two Algorithms (2 and 3). Using low values of the transmission power leads to a better behavior of Algorithms 2 and 3 (Graph 4.6.a) when compared to Graph 4.6.c because notification packets are delivered with a higher probability to the platoon (due to the lower ratio of packet collisions caused by the low transmission power value used to send packets to the medium). However, no-relay behaves much worse since this specific value of the transmission power does not reach the entire 
a) $10 \mathrm{dBm}$, Intervehicular distance $25 \mathrm{~m}$

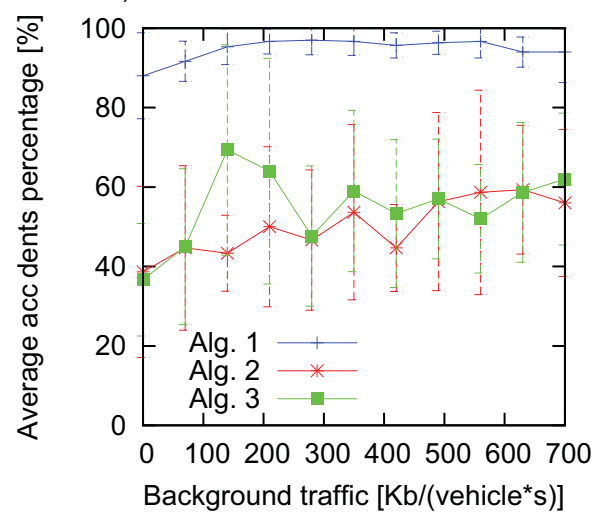

c) $28 \mathrm{dBm}$, Intervehicular distance $25 \mathrm{~m}$

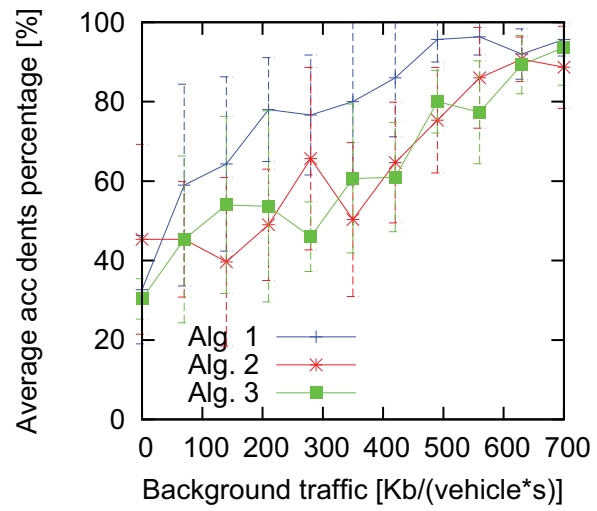

b) $10 \mathrm{dBm}$, Intervehicular distance $60 \mathrm{~m}$

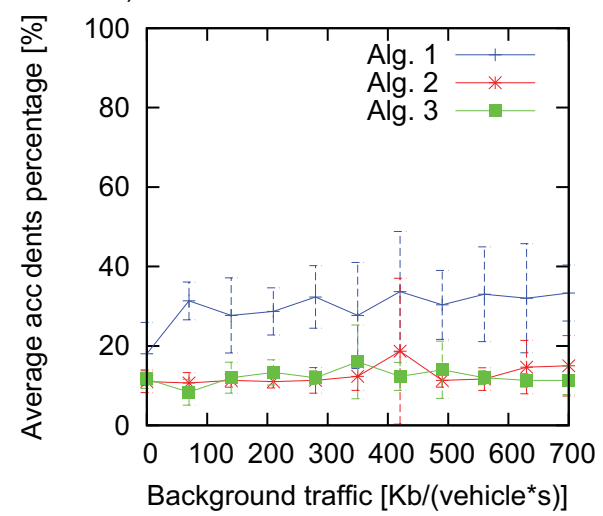

d) $28 \mathrm{dBm}$, Intervehicular distance $60 \mathrm{~m}$

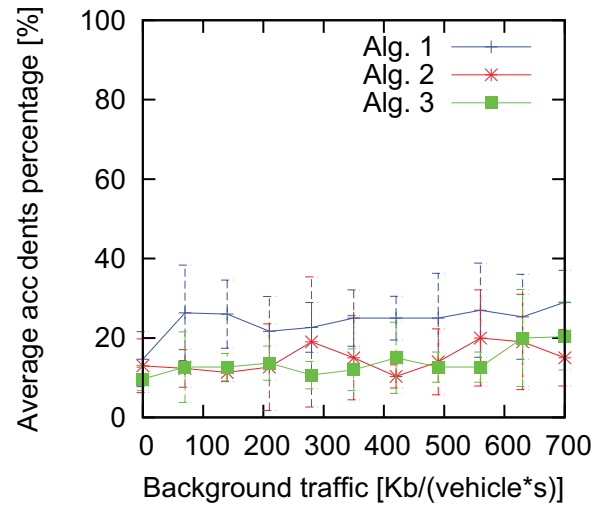

Figure 4.6: Percentage of accidents as a function of the background data traffic delivered by vehicles to the channel

chain of vehicles. In Graph 4.6.c, $28 \mathrm{dBm}$ of transmission power reduces the number of accidents for Algorithm 1 but also has a negative impact on the performance of relay Algorithms 2 and 3 as background data traffic increases. In Table 4.4 we can see in detail the averaged results of the Graphs, showing that Algorithm 3 will normally produce a slightly lower number of accidents due to the less channel overload it causes.

According to previous Graphs in Fig. 4.6 we have noticed that as the transmission power of vehicles increases, the interference between notification packets and background data traffic sent to the environment will also get higher, thus causing a significant growth in the number of car accidents. On the other hand, if transmission power is too low it will take a longer time for vehicles to receive safety information as the 


\section{TESTING VIABILITY OF RELAY POLICIES FOR REACTIVE CCCA APPLICATIONS IN VANETS}

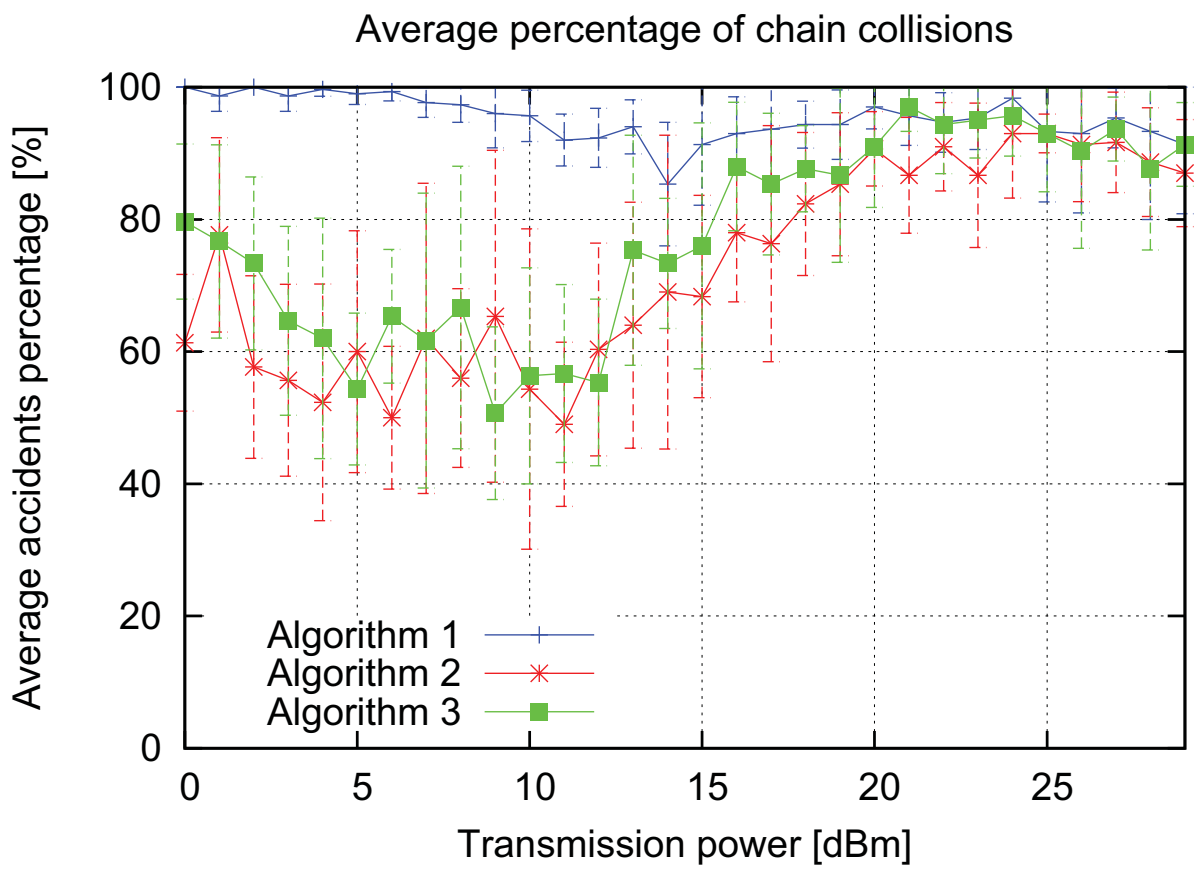

Figure 4.7: Percentage of accidents as a function of the transmission power

Table 4.5: Average accidents percentage for all Transmission Power Values

\begin{tabular}{cccc}
\hline Algorithm & $\mathbf{1}$ & $\mathbf{2}$ & $\mathbf{3}$ \\
\hline Avg. percentage & $95.32 \%$ & $72.82 \%$ & $77.48 \%$ \\
\hline
\end{tabular}

number of hops to reach destination will also be greater, and of course car accidents will consequently increase. As a result, a mechanism to adapt the transmission power according to the background data traffic present in the channel could be a good approach to keep the number of car accidents always low, regardless of the load of the communications channel. In the case in which every vehicle produces a simultaneous background data traffic of $700 \mathrm{~kb} / \mathrm{s}$, we can see in Fig. 4.7 that there seems to be an optimum transmission power value for those cases in which relay Algorithms 2 and 3 are used, which will take a value between 5 and $10 \mathrm{dBm}$. This value depends on the current setting of the platoon of vehicles, the background data traffic and to a minor extent, the relay Algorithm used. In Table 4.5 we can see that in average for the results obtained, only Algorithms 2 and 3 can guarantee a certain reduction in the number of collided vehicles. Even more, Algorithm 2 offers a better result than Algorithm 3 since 
SDR for this case is greater (because more notification messages are retransmitted), although the channel occupation is also higher.

We can conclude from this particular simulation test that background data traffic can be too damaging for safety related packet transmissions in which information must be delivered within the least time affordable. Above all, no-relay can be seriously affected by background data traffic, as could be shown in Fig. 4.6. With relaying, there will be a higher channel occupation, but it will be also guaranteed that packets will reach destination with a higher probability. However, performance of relay Algorithms will be worse as the transmission power is increased, whereas no-relaying mechanisms can perform better in this case when faced against low values of the transmission power.

\subsubsection{Summary of results}

In this subsection, the most relevant results obtained in the present evaluation study are summarized.

- When the channel occupation (in $\mathrm{CCH}$ ) is minimum, one-hop transmissions with enough transmission power values guarantee high SDR in comparison with relay Algorithms, at the same time reducing the channel overload and showing a similar behavior to relay Algorithms as regards the number of car accidents.

- No-relay can guarantee in general lower end-to-end transmission delays (mainly because messages are not retransmitted). This is only effective in those cases where there is no background data traffic sent to the radio channel.

- During the transition phase between $0 \%$ and $100 \%$ technology penetration, norelay behaves very similar to the relay options if transmission power reaches a high enough value (normally the standard value, $28 \mathrm{dBm}$ ).

- Background data traffic affects dramatically the reception of warning notification messages, as can be indirectly deduced from the higher percentage of accidents which take place. There might be a theoretical optimum value for the transmission power which gives us the best trade-off between the lowest channel occupation (for a lower interference) and the SDR (in order to inform vehicles among the shortest interval of time over the incidence), so as to reduce the number of car accidents in the presence of additional background data traffic. 


\section{TESTING VIABILITY OF RELAY POLICIES FOR REACTIVE CCCA APPLICATIONS IN VANETS}

\subsection{Final remarks}

Relaying warning notification messages in CcCA applications is a necessary requirement when the channel's bandwidth is shared with other different applications. Simulation cases in this Chapter have revealed that under circumstances of low channel load, it is enough to use no-relay mechanisms to transmit $\mathrm{CcCA}$ related information to vehicles in the platoon affected by an incidence of collision. Nevertheless, when the level of background data traffic is increased, relay can guarantee a better Successful Delivery Ratio (as shown indirectly by Graphs of Fig. 4.6, due to a lower ratio of car accidents). In this respect, transmission power also plays an important role, since higher values of this magnitude allows for a higher number of vehicles to be under signal coverage. As a result, there is also a higher amount of data packets which are heard by vehicles and thus the packet collision probability also gets increased (more noticeable when comparing relay Algorithms in Graphs 4.6.a and 4.6.c). This makes us believe that there is an optimum value of the transmission power that allows for a correct functionality of the system (minimizing the number of accidents) and at the same time reducing the impact of multiple data packet collisions, which can reduce the Successful Delivery Ratio. A mechanism that can estimate the channel load and provide an optimal value for the transmission power (reducing the channel load as much as possible, but allowing for an acceptable SDR) is a good option to take into account as part of a hypothetical postdoctoral work in order to continue developing improvements for the present reactive $\mathrm{CcCA}$ application. On the other hand, we have assumed for Algorithm 3, that the waiting time interval is always proportional to the sensitivity distance (the distance which in average the transmission power can cover according to the fading model used). It could be a good idea to perform an analysis of the behavior of the SDR and the end-to-end delay according to different configurations of the waiting time interval in Algorithm 3.

In the next Chapter we will study the Intelligent Driver Model (IDM) (18) to improve the realism of results, mainly those recreating human assisted driving. IDM is a simplistic car-following model that is used to describe mobility of vehicles at high speeds in a very reliable fashion. Since in the near future autonomous vehicles will implement support for Automatic Cruise Control (ACC) (as usually called in the literature) for long-duration journeys, it is a good idea to evaluate how communications, 
and in particular, our proposed CcCA application, can be implemented in such models. We will use the tools developed so far, either no-relay as relaying schemes, to integrate anticipation schemes in the aforementioned mobility model, and present a mechanism to reduce driving aggressiveness even under critical emergency braking processes. 
4. TESTING VIABILITY OF RELAY POLICIES FOR REACTIVE CCCA APPLICATIONS IN VANETS 


\section{5}

\section{Evaluating communications and IDM in a context of a CcCA application for VANETs}

\subsection{Introduction}

In Chapters 3 and 4 we have dealt with the simulation and stochastic analysis of scenarios where a CcCA application, both under a simple one-hop retransmission scheme as well as under a relaying approach, was tested. Among other aspects, we compared the performance of our proposal with conventional human-assisted driving in order to extract important remarks about the performance improvement in safety of passengers under dangerous circumstances like the already described. In particular, we implemented a self-made mobility model for human-driven vehicles that consisted of starting to brake after a prudential randomized reaction time had passed since only the directly ahead car started to decelerate (see Fig. A.4 in Appendix A). In reality, humans tend to start pushing the brake pedal not only when the ahead car initiates the deceleration maneuver, but also, when the drivers sense that more vehicles ahead modify their speed. That is, the deceleration policy in general does not depend exclusively on the motion patterns of the ahead neighbor, but to a certain extent also on the other front vehicles. To solve this issue, we can rely on car-following models which recreate these conditions more faithfully, whatsoever needing a multiple-run stochastic simulation approach to implement these schemes (as introduced in the stochastic model of Chapter 3, Section 3.4, this new focus requires Monte-Carlo simulations to obtain results, losing the pure-stochasticity characterization of the initial mathematical model). 


\section{EVALUATING COMMUNICATIONS AND IDM IN A CONTEXT OF A CCCA APPLICATION FOR VANETS}

In this regard, we have chosen the Intelligent Driver Model (IDM), a car-following model extensively studied and evaluated that has been proven to efficiently reproduce car traffic behavior in simulations, and more specifically, in CcCA scenarios. After a coherent calibration of parameters made by its authors (18), the model exhibits its main advantages: it can show very realistically the evolution of mobility of vehicles as a function of time according to several values of the initial boundary conditions (speed, position, acceleration, etc.) by a fixed assignment of the model's physical parameters. In general, when the simulation model is tested against general car-following scenarios, the behavior of vehicles is very realistic, although their steady-state tendency always converges to stable velocities and equal intervehicular distances.

On the other hand, car-following models can also be used to support ACC (Automatic Cruise Control) applications. That is, using sensors to measure the input variables (distance to preceding vehicle, speed difference, etc.) their equations can be used to adapt the speed and acceleration to keep the desired safety gap to the leader car, see (76). In fact, IDM has also been used as an ACC automatic driving guider whose main features were tested in (76). In this evaluation, authors propose an extension to the IDM model as regards an acceleration smoothing heuristic (CAH, Constant Acceleration Heuristic) to avoid the unrealistic behavior of IDM in cases where the accelerations are really high (due to sudden lane changes, or sudden stops by vehicles located ahead). In spite of this fact, the performance of CAH could still be improved if a more specific real-time manipulation of the model parameters was carried out (such as high variations in the deceleration in emergency brake situations).

Vehicular communications have a great potential to improve ACC systems since they remarkably extend the range of the available input and feedback and remove to some extent instabilities caused by parameters such as reaction times. For this reason, in this Chapter we present a proposal to improve safety of passengers when using ACC (with IDM) on critical situations such as CcCA by modifying dynamically the model parameters which influence directly the braking procedure by the use of vehicle to vehicle $(\mathrm{V} 2 \mathrm{~V})$ communications. This way we can also analyze the performance of the already developed policies of our application by using a realistic mobility model like IDM. To the best of the authors knowledge, there has not appeared any evaluation trying to study how communications can influence the behavior of the IDM model (when changing model parameters in real time after receiving warning notification messages), 
and particularly, for the aforementioned situation. Our intention here is to determine in which way it can impact on the best improvement of safety and driver's comfort. Thereby, we propose a braking algorithm which will effectively reduce the probability of accident, and increase the driving comfort (understood as the maximum reduction of the driving style aggressiveness) in such critical situations, thanks to the exploitation of communications. In this chapter, an extensive performance evaluation of the proposed solution will be provided, along with multiple illustrative results which will serve to evaluate how safety can be improved when using already available car-following models by adapting them to the use of communications.

The rest of this Chapter is organized as follows. In the next section relevant related work is described, focusing primarily on the concept of Behavioral Adaptation (BA) (i.e. how general human driving behavior gets influenced by the use of automatic steering assistance devices), and secondly dealing with important questions about the advancement on green consciousness for a cleaner driving. In the following section, our view of communications in IDM is explained; three implementations of the CcCA application (in ACC) are compared together to remark the usefulness of vehicular networks to improve the performance of the IDM model. Then, they are evaluated against our proposed braking algorithm (LBA, Linear Brake Algorithm). Some results and graphs are represented and discussed to illustrate the main features of our proposal. Eventually, the last section closes Part I of the Book by discussing some final remarks on this Chapter and the other two belonging to this Part too (Chapters 3 and 4).

\subsection{Related Work}

When engineers produce and test new technologies, they must always try to construct gadgets and prototypes which behave exactly (or at least with a very good approximation) as they were conceived. For these reasons, designers must deal in parallel with the correct choice of technology, tuning employed devices to work so that performance can be optimized, and analyze how the new technology can introduce changes in the behavior of the driver which could affect overall driving performance. In the particular case of CcCA applications for ACC systems, there are two requirements which the system must necessarily satisfy: reduce the probability of accident with severe consequences, and increase the driving comfort (namely, reducing the driving aggressiveness). To this 


\section{EVALUATING COMMUNICATIONS AND IDM IN A CONTEXT OF A CCCA APPLICATION FOR VANETS}

aim, designers must deal first of all with what is referred as Behavioral Adaptation (BA).

BA involves how Intelligent Transportation Systems (ITS) induce behavioral adaptation in drivers, mainly due to changes in their driving style, risk detection and hazard treatment. This concept has not been treated in detail so far by the industry, since engineers, designers and developers have always assumed that any single technology which can improve mechanical response to a traffic event on vehicles would always mean better driving conditions for drivers. However, it is not always true. That is the case, for example, of Rudin-Brown et al. (77), where authors show that at first glance ACC systems can reduce drivers workload (reliance by them on the technology is significantly increased), but the induced distractions can invalidate some optimistic engineering considerations which assure that car-collision frequency and severity are reduced. They propose to benefit from the so-called training programs to improve human response to avoid this safety issue. In (78), the same authors give some hints to face these already mentioned problems associated to BA. They suggest implementing adaptive interfaces which can, either explicitly or implicitly, learn from the driver about the conditions associated to his/her driving style. This will undoubtedly generate new information which will be used to better understand the reaction of the driver to a certain situation and simultaneously feedback her/him with instructions on how to improve her/his behavior at certain traffic events. In the case of ACC systems (see (78)) some strategies are considered, like for example temporarily disabling the interface whenever the smart system notices larger reaction times to events on the road and longer periods of distraction by the driver. Work in (79) evaluates the causes by which drivers alternate between active and inactive ACC in vehicles. They characterize the system and human behavior by measuring some metrics like mean Time Headway (THW) and Relative Differential Speed (RDS) before and after a transition from one state to another state of the ACC system. Briefly, this study concluded that drivers tend to overrule the ACC when they need an extra acceleration (when with the ACC alone it is not possible to obtain), or when the intervehicular distance is too short in dense traffic and ACC alone is not capable of braking enough. Overall-in-time utilization of ACC (without human intervention) has been treated in (76), where authors of IDM propose an extension of the IDM model (the Constant Acceleration Heuristic, CAH), to avoid (or at least reduce) extreme changes in sudden acceleration/deceleration magnitudes which appear 
when vehicles change lanes or when there are critical emergency stops due to car accidents. A smoother driving style is obtained by their heuristic, which they apply for all the possible traffic states. However, using this smoothing scheme in all possible situations does not allow to improve specific patterns of the vehicle mobility which could induce a better driving experience. In our braking procedure for emergency scenarios, we study this in detail, taking into account that, on the one hand, at first it is possible that drivers will not rely the whole time during the journey on the ACC system, as can be shown in (79); and on the other hand, mobility patterns can be specifically modified (according to the particular state of traffic) to induce the best driving experience.

Apart from the concept of Behavioral Adaptation (BA), during the last years, and due to the significant increase in Green Consciousness among the population, new technologies aim at designing products which respect the environment, reducing gas emissions and contributing to a higher environmental wellness. In particular, Green Driving has evolved to be a key issue during the last decade, because of the predictable shortfalls in gas which are foreseen to take place in the near future, and over all, as a result of the widespread phenomena of the climate change. Applying policies to reduce gas emissions in any driving situation minimizes the impact of these problems. Multiple researches have focused on analyzing how the driving style can influence the fuel consumption mostly on normal traffic situations (city, highway), but critical situations like emergency stop have not been taken into account yet. We suppose that under such critical circumstances, the influence of the gas consumption per vehicle when designing policies which minimize the driving aggressiveness while minimizing the probability of accident may be negligible. Anyway, it is intuitive that reducing variations in acceleration even in critical situations like the one we consider here can be beneficial. This will be a probable subject of study in the postdoctoral stage. The work in (80) has evaluated for instance how Traffic-Light-to-Vehicle communications can significantly reduce gas emissions by anticipating drivers' information about the time remaining to turn to a different state. By using a detailed gas emissions model, they discover that it is possible to reach high percentages of savings in energy consumption and gas emissions to the atmosphere when such information is provided to drivers. The expenditure required to add this feature to vehicles and traffic lights is unfortunately expensive, and furthermore, BA has not been yet evaluated (as regards how this could affect pedestrians' safety). On the other hand, talking about initiatives already present in the market, we 


\section{EVALUATING COMMUNICATIONS AND IDM IN A CONTEXT OF A CCCA APPLICATION FOR VANETS}

can find the EcoGyzer (81), which allows drivers to acquaint some driving skills to help reduce the gas emissions to atmosphere by minimizing driver aggressiveness. Thanks to an active adviser system, EcoGyzer technology benefits from smartphones to provide drivers with real time information about the features of their driving style; thus giving them the capability of consciously enhancing its conditions.

Although the concepts presented in this Related Work do not closely match the main concern of this Work, we can conclude directly that increasing driving comfort even under critical circumstances like CcCA does not only help for the driving experience, but might also be useful to reduce gas emissions and fuel consumption. We also noticed in the previous chapters that the transition stage until the technology is completely deployed will require a great effort (see Fig. 3.7 in Chapter 3) to overcome the problems associated to the simultaneous operation of autonomous and human-assisted vehicles. BA, as explained above, will play an important role in these matters, requiring a tremendous effort in order to facilitate this period, making human driving easier, but at the same time without affecting negatively the functionality of autonomous vehicles, and the whole traffic system, in the end. This is still an open issue (16).

\subsection{Communications in IDM}

IDM is a mobility model already tested to work under different traffic conditions, including support to lane change and smoothing of acceleration/deceleration ${ }^{1}$ magnitudes in general emergency brake situations, as it was shown by the simulated approach with ACC support in (76). As they showed, authors propose five traffic regimes in which the three IDM parameters $(a, b$ and $T)$ are used to set up the particular functionality of the model as regards the specific traffic regime in which vehicles operate (acceleration/deceleration policies, intervehicular spacing, etc.).

However, ACC support could provide a much better functionality to the general system if communications were applied, especially for those situations in which the system is affected by a critical event, such as an emergency brake. For example, assuming that the number of accidents is reduced to the minimum physically possible, deceleration values could be assigned to vehicles in such a way that the maximum comfort and extra safety are guaranteed to driver and passengers as an added value (see Fig. 5.1).

\footnotetext{
${ }^{1}$ Note that along this Chapter, the terms acceleration and deceleration will be used indifferently to denote the process of braking after a car accident occurs.
} 


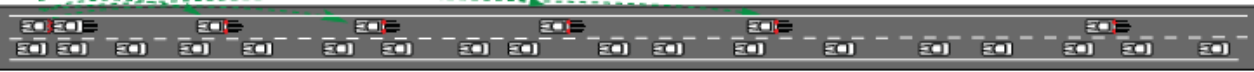

\section{Farthest vehicles collide together and send CWM (Collision Warning Messages) to vehicles located behind to inform about the accident}

Figure 5.1: Critical emergency brake in which communications are used to provide information to anticipate the accident of a leading vehicle

In order to allow IDM to take advantage from communications, we must first interpret the physical/model implications of its most important parameters and the corresponding modifications should be carried out in order to give this support to the model. Let us have a look at the general expression for the calculation of the acceleration in IDM, (82):

$$
\begin{gathered}
\dot{v}_{\alpha}=A\left[1-\left(\frac{v_{\alpha}}{v_{0}}\right)^{4}-\left(\frac{s^{*}\left(v_{\alpha}, \Delta v_{\alpha}\right)}{s_{\alpha}}\right)^{2}\right] \\
s^{*}\left(v_{\alpha}, \Delta v_{\alpha}\right)=s_{0}+v_{\alpha} T+\frac{v_{\alpha} \Delta v_{\alpha}}{2 \sqrt{a b}}
\end{gathered}
$$

where

$\dot{v}_{\alpha}$ : current acceleration of vehicle $\alpha$

$v_{\alpha}$ : current velocity of vehicle $\alpha$

$A$ : acceleration factor

$v_{0}$ : maximum velocity

$s^{*}\left(v_{\alpha}, \Delta v_{\alpha}\right):$ desired safety gap

$s_{\alpha}$ : current intervehicular spacing

$s_{0}$ : minimum intervehicular distance

$\Delta v_{\alpha}:$ speed differential

$a$ : desired acceleration factor

$b$ : desired deceleration factor

$T$ : desired time gap

According to Exp. (5.1), acceleration depends on the speed difference with the leading vehicle, the speed of the current vehicle and the intervehicular distance with 


\section{EVALUATING COMMUNICATIONS AND IDM IN A CONTEXT OF A CCCA APPLICATION FOR VANETS}

the car in front. If we have a detailed glance at the equation, we can see that it can be decomposed into the sum of two expressions:

$$
\dot{v}_{\alpha}=\dot{v}_{\text {free }}+\dot{v}_{\text {brake }}=\left(A\left[1-\left(\frac{v_{\alpha}}{v_{0}}\right)^{4}\right]\right)+\left(-A\left[\left(\frac{s^{*}\left(v_{\alpha}, \Delta v_{\alpha}\right)}{s_{\alpha}}\right)^{2}\right]\right)
$$

where

$\dot{v}_{\text {free }}$ free road acceleration procedure

$\dot{v}_{\text {brake }}$ : deceleration strategy

The second addend $\left(\dot{v}_{\text {brake }}\right)$ in Eq. (5.3) is the relevant one when we talk about hard-braking events in which a critical situation requires vehicles to stop as soon as possible due to a relatively small time gap to the leading car. Under this kind of critical conditions, it is possible to configure dynamically the IDM parameters which determine the behavior of the model in time. The desired minimum gap $(T)$ and the comfortable acceleration/deceleration factors $a$ and $b$ can be thus modified during runtime when a critical situation takes place, especially considering that these three parameters are strongly related to the intervehicular distance a vehicle keeps against its ahead neighbor $(T)$, and the way a vehicle accelerates $(a)$ and decelerates $(b)$ in critical emergency brake situations. Our purpose here is to restrict ourselves to the dynamic configuration of the safety time gap $T$ to help vehicles react according to the braking algorithm we propose, and evaluate how it performs under different circumstances of sudden braking events when compared with other proposed smoothing deceleration algorithms, (76). Our particular focus on the $T$ parameter can be explained assuming that the main intention is to simplify the dynamic scheme process. Taking into consideration parameters $a$ and $b$ would mean many design variables which make the procedure development more intricate.

\subsubsection{General Scenario Description}

In this subsection we introduce the main scenario used to evaluate the performance of the IDM model with the different implementation variants which will be tested to optimize our proposal for the braking procedure. First, we briefly show here how communications can obviously improve the performance of a CcCA application (it could 
be seen in previous chapters). Then, we present the new LBA algorithm to smooth the braking maneuver during an emergency event.

Our general scenario consists of 21 vehicles in a platoon, all of them behaving according to the IDM Model's Eq. (5.1). The initial configuration for the scenario is set for vehicles to keep at the beginning of the simulation a intervehicular distance taken from an exponential distribution (61), with an average gap ranging from 6 to $70 \mathrm{~m}$ in steps of $4 \mathrm{~m}$. Initial speeds for all vehicles are equal and set to $30 \mathrm{~m} / \mathrm{s}$ (at low distances, initial speeds of $30 \mathrm{~m} / \mathrm{s}$ could seem unrealistic, mostly in highways, but this allows us to estimate a lower bound in order to evaluate such extreme event in detail). After a period of $20 \mathrm{~s}$ from the beginning of the simulation, vehicle 21 (the leading vehicle of the platoon, see Fig. 5.1) brakes instantaneously and vehicle 20 crashes with it. Before this instant, vehicles drive in equilibrium (null accelerations and equal intervehicular distances). When the first car crash takes place, information in this regard is sent to further vehicles, entailing an automatic car brake procedure in them to avoid or at least reduce the impact of new hypothetical vehicle collisions. We can see that the CcCA procedure as proposed in this chapter is the same as in the previous two chapters, but with a modified mobility model, given by the Model's Eq. (5.1). Reaction times are defined for each simulation case in particular.

For those cases in which communications apply, the Nakagami channel propagation fading model is used for the transmission of information, see (83). Transmissionreception delay of messages can be ignored (since we assume that the communications channel is not shared with other applications).

Simulation parameters whose values keep constant for all simulation cases can be seen in Table 5.1. For each case, only variable parameters are shown to reduce total writing space. To compare the performance of our proposal against real traffic circumstances, we choose the values for the parameters set up in (18). Initial speeds are set to $30 \mathrm{~m} / \mathrm{s}$ since this is an average value found in highways.

The evolution of the percentage of collided vehicles in the platoon is shown for the range of intervehicular distances mentioned before. We also represent the average variance of the braking decelerations that vehicles use to decrease speed to a complete stop versus every intervehicular distance. The purpose of representing this evolution is to provide further information regarding the associated driving aggressiveness related to the corresponding deceleration algorithm employed (when it applies), in order to 


\section{EVALUATING COMMUNICATIONS AND IDM IN A CONTEXT OF A CCCA APPLICATION FOR VANETS}

Table 5.1: Configuration parameters for all simulations

\begin{tabular}{cc}
\hline Model parameters & Values \\
\hline$A$ & $1 \mathrm{~m} / \mathrm{s}^{2}$ \\
$b$ & $1.5 \mathrm{~m} / \mathrm{s}^{2}$ \\
$s_{0}$ & $2 \mathrm{~m}$ \\
$a$ & $1 \mathrm{~m} / \mathrm{s}^{2}$ \\
$v_{\text {ini }}$ & $30 \mathrm{~m} / \mathrm{s}$ \\
$v_{0}$ & $33 \mathrm{~m} / \mathrm{s}$ \\
\hline Simulation parameters & Values \\
\hline$N$ (Number of vehicles) & 21 \\
$a_{\text {stop }}$ (Maximum & $-8 \mathrm{~m} / \mathrm{s}^{2}$ \\
deceleration value) & \\
$d$ (Intervehicle distance & {$[6-70] \mathrm{m}$} \\
range) for exponential & \\
distribution &
\end{tabular}

give a general qualitative view of the comfort offered to passengers by the respective braking procedure. According to (84), maximum comfort is obtained when changes in braking decelerations to reach the final traffic state (all cars stopped) are reduced to the minimum physically affordable.

In some illustrative cases we represent the average final speed to stop for all vehicles (20 samples), when the intervehicular distance is $6 \mathrm{~m}$, along with the minimum theoretical obtainable values of speeds to stop, see Fig. 5.3 for an example. Theoretical minimum speeds are defined as the speed the vehicles will reach when they stop completely (either by crashing or stopping successfully) if they react to the first accident by braking at the maximum deceleration physically obtainable $\left(8 \mathrm{~m} / \mathrm{s}^{2}\right)$ with null reaction time (vehicles begin to decelerate at the same time instant the first accident takes place). The use of a maximum theoretical deceleration value of $8 \mathrm{~m} / \mathrm{s}^{2}$ can be justified assuming that it acts as an upper reference (in reality, vehicles achieve such value under very favorable road, driver and environmental conditions). In red we show the averaged simulation speeds when stopping versus the minimum theoretical speeds (in blue). Simulations are performed and shown with a $99 \%$ confidence interval for all the statistics we measure. 


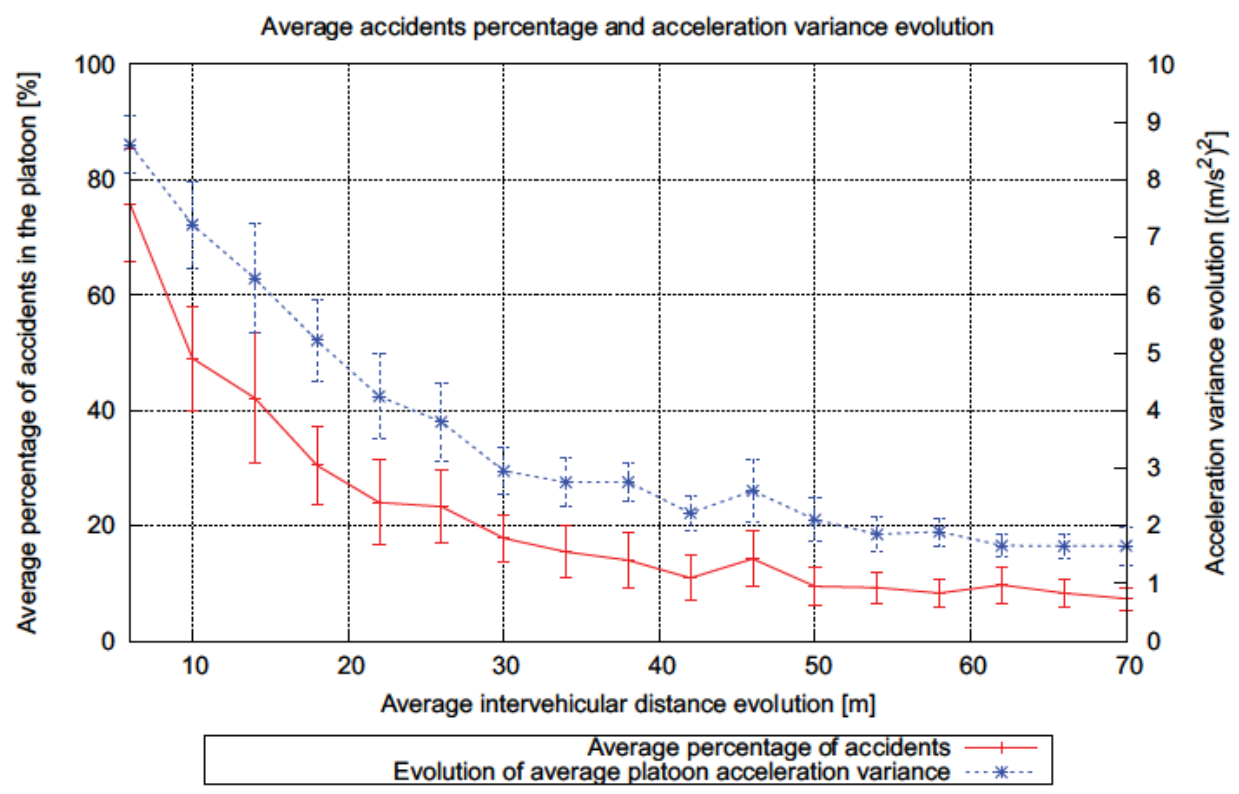

Figure 5.2: Average percentage of accidents in the platoon (left axis) and evolution of average acceleration variance of vehicles in the platoon (right axis)

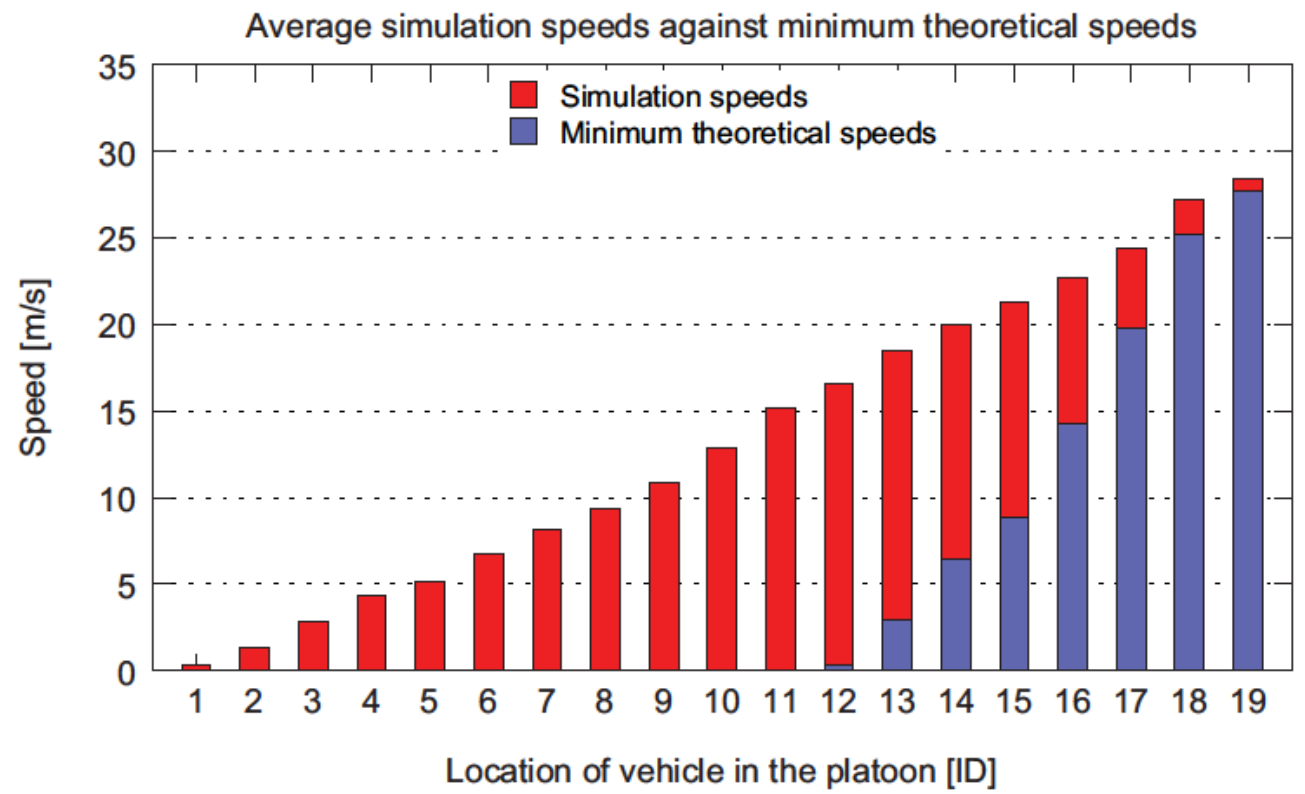

Figure 5.3: Minimum obtainable speeds to stop (blue) versus simulation speeds (red) to stop for an average intervehicular distance of 6 meters 


\section{EVALUATING COMMUNICATIONS AND IDM IN A CONTEXT OF A CCCA APPLICATION FOR VANETS}

\subsubsection{Need for communications}

Now, we discuss the advantage of using a system which supports vehicular communications to reduce the number of car accidents (as a prologue to the definition of our braking procedure). When communications are not in use, microscopic mobility patterns are calculated according to the general Exp. in (5.1). In this equation, vehicles react to the changes of the vehicle located ahead. This entails a chain induced reaction, in which there is an obvious incremental delay from the instant in which the first vehicle collides until the last car in the chain begins to decelerate due to the platoon's leading vehicle which crashed first. To show this, we conduct a simulation supporting IDM with null reaction time, along with general values for the model parameters in Table 5.1. The platoon is composed of 21 vehicles (as in the general scenario description), the maximum deceleration value is $-8 \mathrm{~m} / \mathrm{s}^{2}$ (as a physical upper value for the maximum deceleration), and the reaction time is set to $0 \mathrm{~s}$ because we are using IDM (however, since the sampling period of the network simulator is established to $S=100 \mathrm{~ms}$ (see Appendix A), the delay to react to phenomena is intrinsically $0.1 \mathrm{~s}$, which is used to model the turn-around time regarding reception and packet processing of OBUs in vehicles).

If we observe Fig. 5.2, we can notice that even though reaction time is very small (approximately the time taken by the ACC system to account for the change of the speed of ahead vehicles), when the average intervehicular distance is $6 \mathrm{~m}$, more than $75 \%$ of the vehicles of the platoon still collide (this metric decreases obviously as the average intervehicular gap increases). In Fig. 5.3 we show the speeds of the vehicles in the platoon when stopping, averaged with 20 simulation runs and for a mean intervehicular distance of $6 \mathrm{~m}$. The red bars in Fig. 5.3 show that there is a large amount of vehicles (mainly located at the middle of the platoon) which could have stopped previously, thus reducing the average speed to stop (or even avoiding collision, which happens when the speed is null), mainly if some kind of communications system were used to inform them earlier about the incidence. As we can easily realize, this high value in the average final speed of vehicles in the middle of the chain corresponds to the same phenomena extracted from the central elbow as seen, for example, in Figs. 3.3 and 3.7 in Chapter 3. We have chosen the short distance of $6 \mathrm{~m}$ for the intervehicular 
Table 5.2: Characterization of transmission packets

\begin{tabular}{ccc}
\hline & Beacons & CWM \\
\hline Purpose & $\begin{array}{c}\text { Transmission of } \\
\text { general mobility } \\
\text { information }\end{array}$ & $\begin{array}{c}\text { Transmission of } \\
\text { accident related } \\
\text { information }\end{array}$ \\
\hline Type & Periodic & Event-driven \\
\hline Position $[\mathrm{m}]$ & $\nabla$ & $\nabla$ \\
Speed $[\mathrm{m} / \mathrm{s}]$ & $\nabla$ & $\boldsymbol{\nabla}$ \\
Acceleration & $\nabla$ & $\boldsymbol{x}$ \\
{$\left[\mathrm{m} / \mathrm{s}^{2}\right]$} & $\nabla$ & $\nabla$ \\
Timestamp $[\mathrm{s}]$ & $\boldsymbol{\nabla}$ & $\nabla$ \\
Priority flag & &
\end{tabular}

distance because it is a worst-case scenario (differential speed between theoretical and simulated speed is smaller as the intervehicular distance is increased).

\subsubsection{Communications in IDM: $T$ conservative approach}

As it was observed in the previous subsection, some kind of system to anticipate information to vehicles facing a critical event like an emergency brake is beneficial to mitigate the impact of chain collisions in a context of Cooperative chain Collision Avoidance (CcCA). The communication system's main purpose consists of beaconing status information periodically to inform about the mobility data of every vehicle. Whenever an unpredictable event takes place (i.e. an accident) beacons are replaced by collision warning messages (CWM), with higher priority, which will be sent to the remaining vehicles in the chain (only by collided cars, periodically after the emergency incident). The way in which these messages are treated (how they affect to the model parameters $a, b$ and $T$ ) will influence the braking procedure of every vehicle until a complete stop. The contents of beacons and CWM are detailed in Table 5.2.

Position, speed and acceleration fields inform about the mobility patterns associated to the sender, while the Timestamp registers the time in which this information was recorded. The priority flag denotes the importance of the message (1 maximum, 0 normal). With these data, vehicles will have enough information to react earlier in order to perform a complete stop, thus reducing the probability of crashing. To define the main features of our particular braking algorithm we first find out how is the 


\section{EVALUATING COMMUNICATIONS AND IDM IN A CONTEXT OF A CCCA APPLICATION FOR VANETS}

Table 5.3: Specific simulation parameters for the $T$ conservative approach

\begin{tabular}{cc}
\hline $\begin{array}{c}\text { Model and simulation } \\
\text { parameters }\end{array}$ & Values \\
\hline$T$ & $1.5 \mathrm{~s}$ (constant) \\
$T_{r}$ (reaction time) & $0 \mathrm{~s}$ \\
\hline
\end{tabular}

behavior of the system supporting communications without a dynamic configuration of the IDM parameter $T$, that is to say, this parameter will take for all vehicles a more conservative value at the time a CWM is received (which will make all vehicles increase the distance respect to their frontal one). The main reason for this test is to evaluate the performance of a general approach in which vehicles tend to keep a conservative distance to neighbors ahead when they receive a CWM, without taking into account the associated driving aggressiveness in the protocol design. Configuration values for the present simulation are summarized in Table 5.3.

As can be seen from Figs. 5.4 and 5.5 (and comparing them with Figs. 5.2 and 5.3 ), the system obviously behaves much better when communications are used, because vehicles can react to the incident earlier, adopting a remarkable conservative approach respect to ahead vehicles. This helps to reduce the probability of getting involved in an accident: around $50 \%$ less accidents with communications (when compared to Simulation Case 1, without communications support). On the other hand, if we observe the evolution of the acceleration variance when braking proceeds, values tend to be notably high (very similar to those of Simulation Case 1). This implies that although car collisions are reduced, accelerations employed to brake to a complete stop are still inadequate, since extreme variations in the braking acceleration show that the braking procedure could be effectively improved (reaching the same average acceleration to a complete stop, but minimizing the variations in acceleration). This would enhance the driving experience even under these critical situations, since driver and passengers would not be exposed to so high acceleration variations, meaning a better and more comfortable braking process (of course, in those cases in which it is physically possible).

\subsubsection{Linear Braking Algorithm (LBA)}

After introducing the main technical aspects related to the braking procedure, as well as the communications' protocol associated, we will perform a simulation of the pro- 


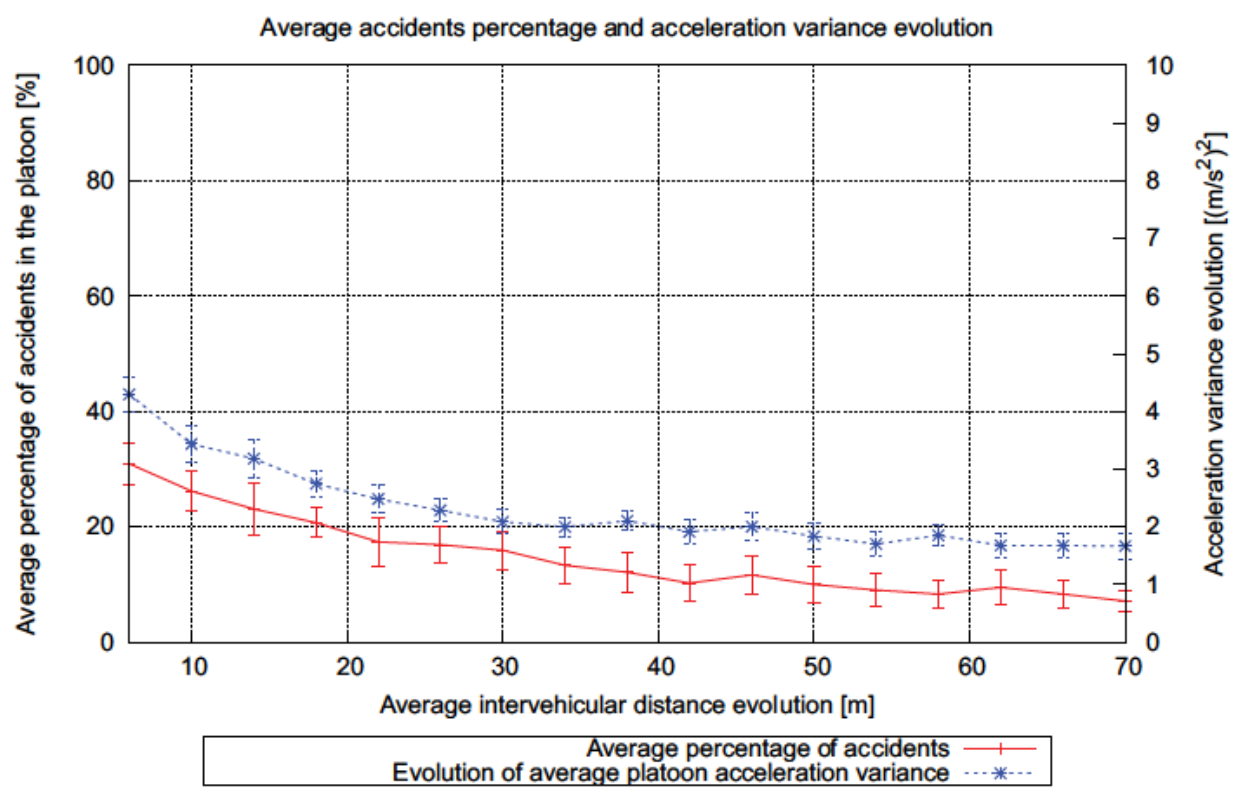

Figure 5.4: Average percentage of accidents within the platoon (left axis) and evolution of average acceleration variance of vehicles in the platoon (right axis)

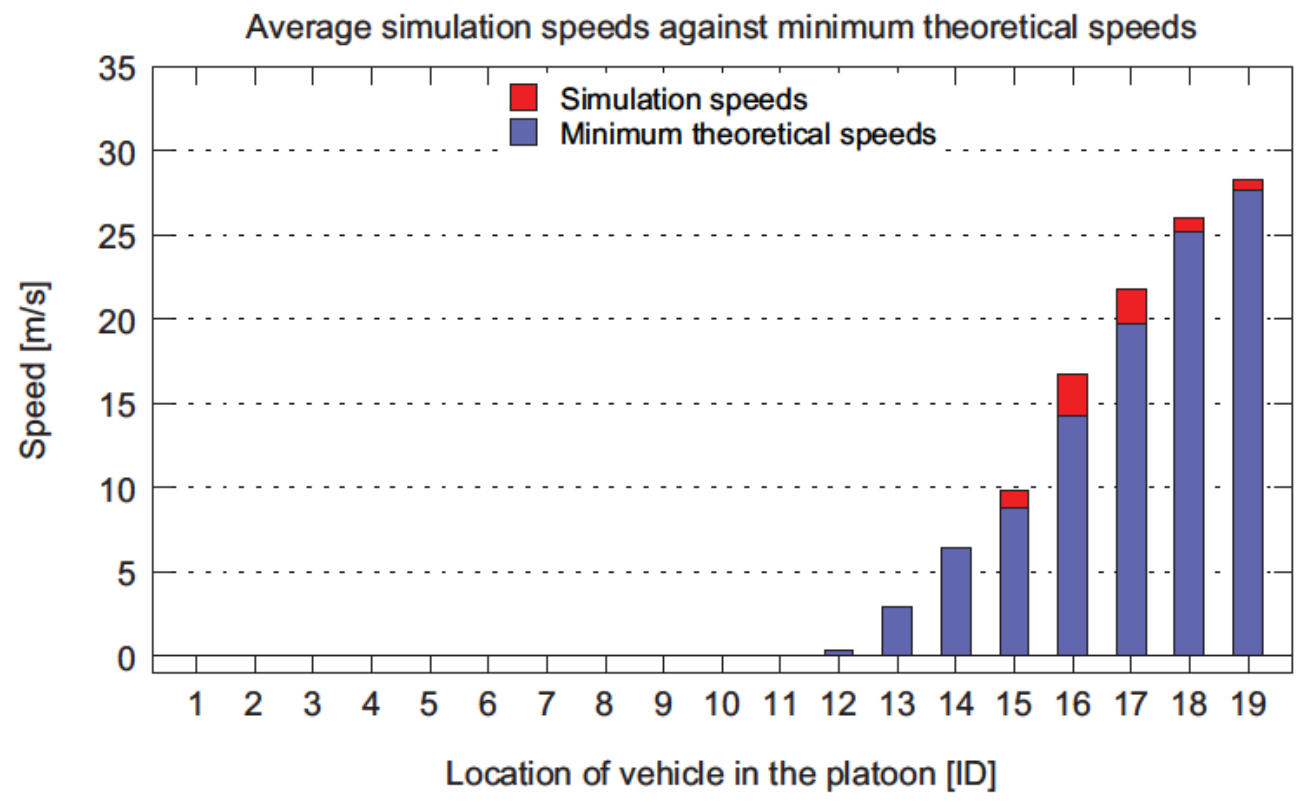

Figure 5.5: Minimum obtainable speeds to stop versus simulation speeds to stop for an average intervehicular distance of 6 meters

posed braking scheme and represent its results. Another simulation regarding the $\mathrm{CAH}$ (constant acceleration heuristic), will be carried out to compare the performance of our 


\section{EVALUATING COMMUNICATIONS AND IDM IN A CONTEXT OF A CCCA APPLICATION FOR VANETS}

proposal with this smoothing acceleration/deceleration procedure already presented in other work by the authors of the IDM, (76). For a fair comparison, the CAH will rely on communications as well, working only when a CWM is received (accelerations according to this heuristic will be calculated only when $\mathrm{CcCA}$ is triggered). The main functionality of $\mathrm{CAH}$ is described in the Algorithm 5. Two graphs, as regards the Average acceleration variance evolution and the Average percentage of accidents in the platoon will be represented, to compare the four cases illustrated in this work together.

By designing a Linear Braking Algorithm (LBA) we mean to configure dynamically the braking scheme of vehicles which must deal with a critical situation such as an emergency stop. The main purpose has been already mentioned: to decrease or smooth the changes in acceleration and, as a result, obtain a reduction in the driving aggressiveness as well. Communications receive a major attention here, since they allow vehicles to own and use relevant and updated information about their neighbors. When treated correctly, in a system like CcCA (under the consideration of the IDM model), it can be really useful, as we have seen, not only for the reduction of the probability of accident, but also for the best improvement of the driving comfort even for extreme conditions of the vehicular traffic. In LBA, vehicles interchange beacons periodically, informing neighbors about their associated mobility patterns: acceleration, speed and position, and the related timestamp of these values. This way, vehicles will know in real time the mobility patterns of their surrounding neighbors. Assuming that driving is human-assisted before the critical event takes place, when this happens, CWMs are sent to inform about the incidence occurred. Thanks to the information provided by beacons and CWMs, it is possible to design a dynamic braking scheme which can help vehicles stop under a quasi-constant deceleration value (without human intervention). The main algorithm for the CcCA support of IDM in this work is explained in the next paragraph (see also Algorithm 4).

A vehicle will circulate on the road sending periodically state information regarding its main mobility parameters. If a beacon is received, the receiver will update its status table with new neighbors' information (position, speed and acceleration) according to the data contained in the message. If a vehicle receives a CWM, it calculates the LBA deceleration value to stop without colliding with neighbors ahead and to reduce the driving aggressiveness. Knowing the information related to vehicles in front and between the vehicle under consideration and the first collided car, the receiver computes 

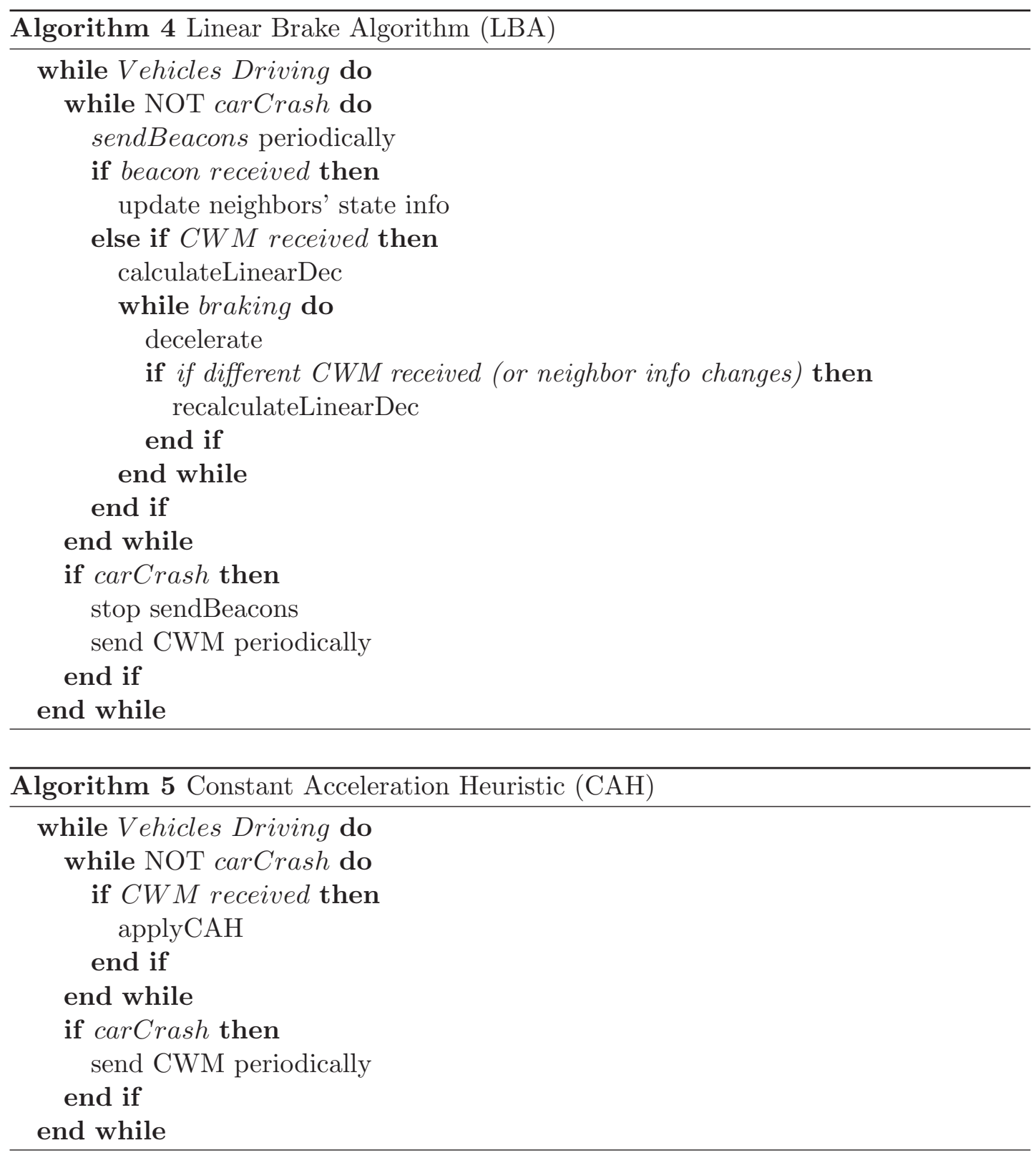

an estimation of what is the position in which it should stop completely, obtaining thus the deceleration value necessary to brake to a complete stop without changing the deceleration while braking, that is, with constant deceleration. If the mobility patterns of vehicles located ahead (namely deceleration) change during the braking procedure, the vehicle will recalculate its deceleration value, to deal with unforeseen changes which could naturally occur in the emergency scenario. Afterwards, the $T$ 


\section{EVALUATING COMMUNICATIONS AND IDM IN A CONTEXT OF A CCCA APPLICATION FOR VANETS}

parameter is calculated according to the general equation which solves the safety time gap value $T$ according to the general mobility parameters. This parameter will be calculated at every time step to keep the deceleration value constant and equal to the one calculated previously, Eq. (5.4):

$$
T=\frac{\sqrt{(-1)\left[a_{i}(t)-A+A\left(\frac{v_{\alpha}}{v_{0}}\right)^{\delta}\right] \frac{s_{\alpha}^{2}}{A}}-s_{0}-\left(v_{\alpha} \Delta v_{\alpha}\right)}{v_{\alpha} 2 \sqrt{a b}}
$$

where

$$
\begin{aligned}
& v_{\alpha}: \text { current velocity of vehicle } \alpha \\
& A \text { : acceleration factor } \\
& v_{0}: \text { maximum velocity } \\
& s_{\alpha}: \text { current intervehicular spacing } \\
& s_{0}: \text { minimum intervehicular distance } \\
& a_{i}(t) \text { : current acceleration of vehicle } i \\
& \Delta v_{\alpha}: \text { speed differential } \\
& a \text { : desired acceleration factor } \\
& b: \text { desired deceleration factor } \\
& T \text { : desired time gap }
\end{aligned}
$$

To test the behavior of our scheme, we compare the two previous cases with it and the acceleration-smoothing algorithm $\mathrm{CAH}$ proposed by (76). If we observe the evolution of the number of car accidents in the platoon for the different intervehicular distances (Fig. 5.6), we can notice the great difference in performance between using and not using communications in the IDM model. When there is no communications support, at low intervehicular distances $(6 \mathrm{~m})$ the percentage of accidents reaches almost the $80 \%$ of the platoon. On the other hand, regardless of the braking procedure used, the probability of accident is reduced to the half (mainly at lower distances) when communications are used, which implies a remarkably higher guarantee of safety for drivers and passengers. At higher distances, results for all schemes are the same (Fig. 5.6). This behavior can be explained assuming that vehicles keep large enough distances to neighbors ahead, so that it is possible to brake timely in order to avoid crashing. The percentage of accidents appearing at these high distances corresponds to those vehicles 
sufficiently close to the first accident, which do not have the chance of stopping without colliding.

However, when we evaluate the driving aggressiveness implied by all the braking procedures, it is clearly shown that there are some differences between each single approach (Fig. 5.7). The plain IDM behaves noticeably much worse than the rest of the cases. It is caused by the fact that without communications support, vehicles tend to react later to the sudden brake of neighbors ahead. When communications are operating, in the case in which a constant (conservative) value of $T=1.5 \mathrm{~s}$ is employed when a CWM is received, the acceleration variance is reduced by half for low distances (6,10 and $14 \mathrm{~m}$ of intervehicular distance). CAH also succeeds in smoothing deceleration changes by getting (little) lower values for the acceleration variance. Nevertheless, if we look carefully at the evolution achieved by the LBA proposal we can notice that configuring mobility parameters in such a way that braking deceleration is kept quasi-constant during the whole emergency procedure, means obtaining a very low acceleration variance which obviously implies higher driving comfort. Thereby it is possible to reduce the number of car accidents in the platoon (Fig. 5.6), and simultaneously decrease the driving aggressiveness, which is actually a very important design concern for the vehicle industry (85).

\subsection{Final remarks}

As the previous sections reveal, the design of advanced braking policies is essential to cover all possible car traffic situations in order to assure the best performance and safety guarantees while driving. IDM, as regards the particular traffic configurations provided in the evaluations in (76), can achieve a very realistic performance in terms of safety, driving aggressiveness and general car traffic throughput. However, the coverage is very general, and under certain specific circumstances the model can miss a better performance which could be obtained if a more detailed manipulation of the model parameters was taken (see Fig. 5.7). In this Chapter in particular, we have shown that during emergency brake situations, it is possible to reduce the number of accidents in a platoon by using a car-following model like IDM for ACC support, while simultaneously decreasing the driving aggressiveness of drivers. Our Linear Brake Algorithm (LBA) enables the possibility to benefit from communications under emergency brake 


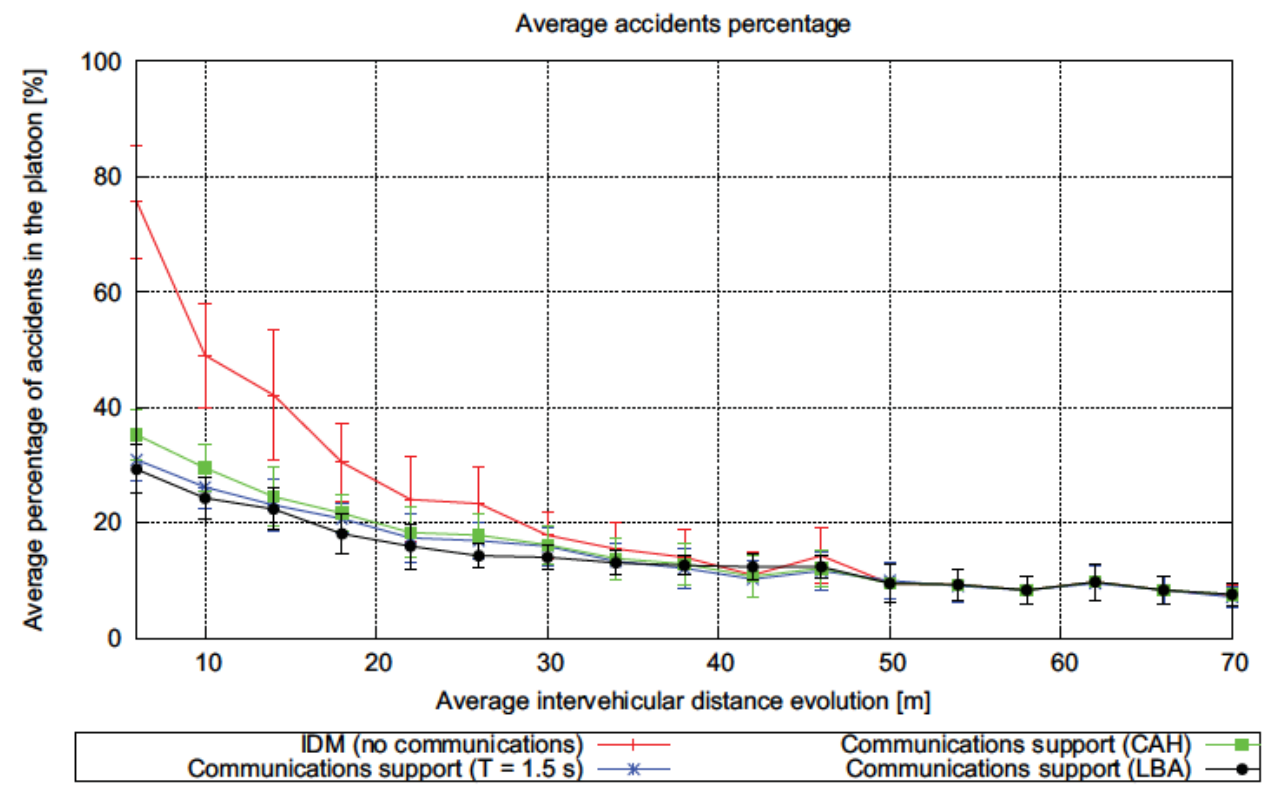

Figure 5.6: Evolution of average accidents percentage for the four implementation variants studied in this work

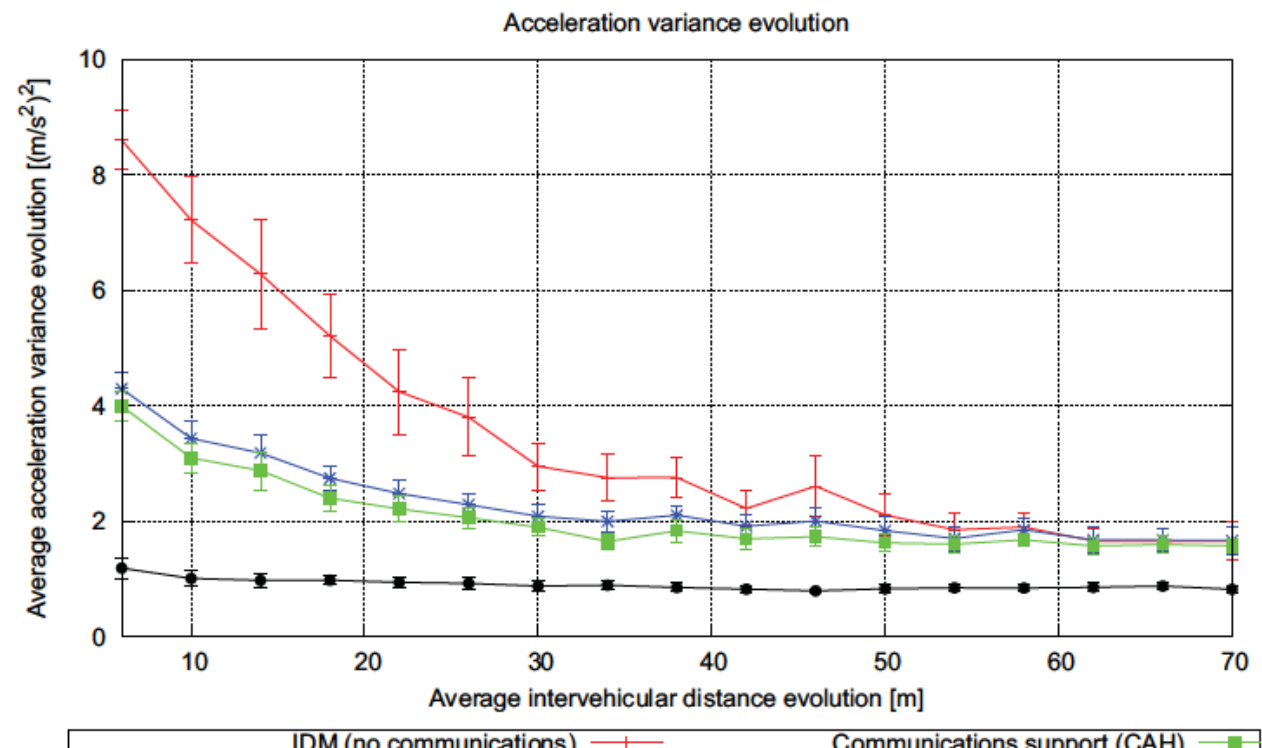

Figure 5.7: Evolution of acceleration variance for the four implementation variants studied in this work 
conditions to reduce simultaneously the impact on the number of car accidents and the driving aggressiveness of the vehicle. This enhancement in the driving comfort will have positive health implications in passengers (since they will be exposed to lower braking decelerations as well as less changes in their magnitudes), along with minor gas consumptions and brake deterioration. Taking into account the previous results, acceptable scalability can be obtained in simulations when general configuration parameters $(a, b$ and $T)$ are assigned to the different traffic regimes in IDM. However, it is necessary to work on specific schemes to deal with those specific circumstances where a better behavior of the system could be achieved if a particular configuration of such parameters was made.

Unfortunately, integrating communications into IDM and modifying dynamically the $T$ parameter unavoidably has two important drawbacks: sensitivity of parameters to the changes, and the problem associated to the $T$ parameter when the speed of a vehicle tends to zero ( $T$ value tends to infinite, see Eq. (5.4)). In order to get rid of these two inconsistencies, one solution could be to analyze these issues more closely. Furthermore, the model parameters $a$ and $b$ might be also useful to better improve the braking algorithm and reduce the effect of the two aforementioned inconsistencies related to parameters sensitivity and the $T$-infinite trend when approaching zero speed. Whatsoever, already available technologies for ACC are conceived to work under stable circumstances of the car traffic, thus although the main aim of using IDM was to compare functionality of this model with our self-developed mobility model of vehicles (as introduced in Appendix A), the utilization of IDM (despite with communications' support) might be unrealistic in CcCA situations. Anyway, we could derive some useful conclusions as could be exposed in the earlier paragraph, which justify the premise of driving aggressiveness minimization even under this sort of critical circumstances. In Part III we will change the paradigm and investigate on how to design a procedure to obtain real-time evasive trajectories for CCA applications, by simultaneously taking into account how the impact of mobility on driving aggressiveness can be reduced. 
5. EVALUATING COMMUNICATIONS AND IDM IN A CONTEXT OF A CCCA APPLICATION FOR VANETS 


\section{Part III}

\section{Cooperative Collision Avoidance by evasive maneuvering}





\section{6}

\section{Cooperative Collision Avoidance by active evasive maneuvering}

\subsection{Introduction}

During the first part of this Thesis we have dealt with the characterization and study of how communications can help reduce the risk of accident when vehicle platoons are exposed to events of multiple chain collisions. We assumed that in these cases vehicles did not have enough time to make alternative evasive routes to avoid the collision, so the only chance was to execute a braking maneuver in order to avoid the imminent crash with ahead vehicles. However, it is possible that there might be certain situations in which vehicles could have enough time and lateral space when they are approaching a traffic jam ahead, and then use this free gap as an additional space to avoid further collisions with stopped vehicles, or even with other sort of obstacles (animals, pedestrians...) standing on the road (see Fig. 6.1).

In this chapter we propose a complete procedure for the computation of trajectories for vehicles that can benefit from these lateral gaps to reduce the probability of crashing. We pose this as an optimal control problem and simplify it by considering only lateral motion, thus having to solve a one-dimensional trajectory tracing problem. This simplification is brought up as a worst case scenario where vehicles have to share the lateral space to overcome the obstacles appearing ahead.

We introduce a general performance measure including three different optimization criteria to evaluate the goodness of the possible trajectories as follows. First, from the point of view of safety, vehicles should keep a maximum separation between them and 


\section{COOPERATIVE COLLISION AVOIDANCE BY ACTIVE EVASIVE MANEUVERING}

the obstacles at the instant they cross the obstacle's position. Second, it is important to control (minimize) the lateral speed at the moment of overcoming the obstacles. That is, we could find a trajectory that maximizes the lateral distance with the obstacle at the moment of reaching its position, but if it has a non-zero lateral speed it might result in a later collision with the lateral barriers or another vehicle. There may be a trade-off between both goals as we will show. Finally, to minimize the impact of sudden accelerations on the passengers, the lateral acceleration evolution during the maneuver should be also controlled.

The optimal control problem is solved by applying an optimization strategy that makes use of a modified version of the Gradient Projection method (86) (which we call Modified Gradient Projection method, and refer to it as MGP from now on). The reason is that the lateral distance component makes the functional non-differentiable. Actually, this strategy divides the problem into a set of separate subproblems with differentiable functionals that are minimized by using the MGP method. However, each subproblem is non-convex, so one has to choose carefully the initial guess trajectory to perform the gradient descent with the MGP method because different initial values might turn into different solutions. However, it does not require a high processing power to compute such trajectories, since in practical terms the number of subproblems to solve is relatively small ${ }^{1}$. The utility of this method is justified by accounting for the recommendations to decrease the processing cost for this kind of ITS applications since they usually imply very high workloads (LIDAR sensors and 3D-image processing (39)) that have to be processed at minimum time. We discuss first these issues in Subsection 6.3.3, and leave the main details of the optimization strategy to the Subsection 6.3.5. On the other hand, the algorithmic structure of the MGP method is introduced in Subsection 6.3.4. To end this chapter, we will illustrate some results to show the validity of our approach and its flexibility to compute evasive trajectories adapted to different requirements, leaving the particular operations needed to obtain some of the mathematical expressions of this chapter to Appendix C. As additional material, we will use Appendix D to conduct a study regarding the computation of optimum trajectories for vehicles under additive Gaussian noise, and how these inconveniences can be minimized by making use of Kalman Filters (42).

\footnotetext{
${ }^{1}$ It depends on the number of vehicles and objects participating in the problem, and in real world situations this is not high in general.
} 


\subsection{Related Work}

When characterizing different optimum trajectory tracing schemes, the specification of the performance criteria plays a major role. If we begin by focusing on tracking error minimization (between the desired and the followed route), one relevant work regarding optimum lane tracking was conducted by Fenton (87). In that work, different controller designs were tested for the lateral motion control of a vehicle on a lanedependent coordinate system. Also, an extension of this work was later presented by the same author in (88), where an updated speed-adaptive version of the main controllers was given, increasing robustness, accuracy and ride comfort during the lane tracking process. Although not in tight correspondence with our application's main purpose (evasive maneuvering), both works justify the presence of feedback controllers to constantly correct the lateral position due to unpredictable disturbances. A similar approach was carried out by the work of Tomizuka (89), which relies on the same state equations that we propose in our work, but performing lane tracking with error minimization. By determining a desired given trajectory to be traced after the present moment $t_{0}$, they evaluate the influence of an additive Gaussian noise and how this can be mitigated by making use of the past control history with Kalman filtering (42). On optimum lane changing (where preview trajectories are calculated to carry out laneto-lane transitions) we can highlight the work of Chee (90), where the performance evaluation of some controllers was carried to test their performance when tracking a VDT (Virtual Desired Trajectory), whose shape is curiously identical to the trajectories traced by our algorithm when minimizing the final lateral speed (see Section 6.4).

Another approach for the optimization criteria can be to cover a trajectory in minimum time. That is the case of the mathematical study in (91), where Venkatraman et al. presented some results on how to trace optimum trajectories in minimum time until a final heading is reached. Another interesting approach is found in (92), where a nearly-time optimal control for a Dubin's car model (93) is proposed in order to complete paths between two pre-established positions in space. The nearly-time optimality avoids bang-bang ${ }^{1}$ controls in order to improve the convergence of the optimization algorithm at the expense of not reaching the last position while minimizing the time period. Another work worth mentioning was proposed by Godbole et al. (94). They

\footnotetext{
${ }^{1}$ Bang-bang trajectories are those which oscillate between the two extreme values of the controls to optimize the paths (see (19)).
} 


\section{COOPERATIVE COLLISION AVOIDANCE BY ACTIVE EVASIVE MANEUVERING}

provided an optimum lane change scheme for collision avoidance in a specific scenario, where vehicles in the destination lane leave space in-between to allow the vehicle executing the maneuver fit between them and avoid the collision with an object ahead on the same lane. Depending on the time available to perform the operation, this will have to be executed in minimum time at the expense of degrading driving comfort, or using a smoother lane transition for an improved driving experience. The work in (95) also focused on comfort and provided optimum trajectories for lane-change maneuvers while ensuring overall and maximum lateral acceleration are minimized for a reduction in the inertial effects while lane-changing. They also made a fifth-degree polynomial regression of their trajectories (see (96)) in order to reduce the computational costs of the procedure. As we will see later in our results, the trade-off between minimum time and comfort is also taken into account in our functionals, but for a more general multivehicular worst-case scenario. We can also remark the work of Shamir (97), who showed that minimizing the total kinetic energy during a complete overtaking process (over a slower vehicle) provides time- and arc-length optimum trajectories. Using an advanced mechanical model for the vehicular motion, Dingle dealt in (98) with the calculation of optimum evasive maneuvers for a vehicle so as to avoid a static obstacle by a three-phase trajectory approach as in $(94,97)$. By using an advanced mechanical model for the tyres' response, the work in (98) justified the need to use simpler mobility models to compute such optimum trajectories due to the, even for just one car, high computational costs required. In our case we significantly reduce the complexity of the model by solving a one-dimensional problem in order to calculate optimum cooperative maneuvers for more than one vehicle at a time.

If we now focus directly on literature for obstacle avoidance by threat assessment we can highlight the work of Schmidt et al. (99). They presented a novel approach where the so-called Exclusion Regions are computed in an emergency obstacle avoidance situation to obtain all the possible collision avoidance trajectories that a car could follow. From (99) it is possible to justify the non-convexity of the problem we propose by visualizing the obvious multiplicity of trajectories that a car can take in obstacle avoidance. Only by incorporating additional performance criteria (or constraints) which model the maneuver, we can reduce the number of possible solutions or even come out with a convex problem. Eidehall et al. (100) performed a study about the utility of using the ELA (Electronic Lane Assist) concept when comparing to other 
systems alike. Interestingly, the Collision Avoidance by Steering system (CAbS), for which our proposal provides a possible implementation, is one of the obstacle avoidance safety concepts whose impact on the metric utility/cost of implementation gives the highest ratio. A more recent work was presented by Bränströmm (101), modeling an algorithm to calculate the set of possible maneuvers so as to avoid colliding under critical circumstances in general traffic scenarios by assessing under which conditions autonomous or human intervention should be chosen. Although not tightly related with our study, it is quite interesting to note that in that work both autonomous and human driving are seamlessly integrated, an aspect which is clearly crucial at the early stage of the total penetration of the technology in the market.

\subsection{Problem statement and formulation}

Let us consider a scenario as shown in Fig. 6.1 where a total of $L$ vehicles (a maximum of one vehicle per lane) circulate on a two-dimensional planar road ( $O$ lanes with $W_{l}$ $\mathrm{m}$ of width, external edge $\left(W_{e} \mathrm{~m}\right)$ and internal edge $\left(W_{i} \mathrm{~m}\right)$, where $\left.L \leq O\right)$. Assume that vehicles have width and length dimensions, and that their positions with respect to the given pair of axes are referenced according to the central front part of each one, $\left\{\left[x_{1}(t), y_{1}(t)\right], \ldots,\left[x_{L}(t), y_{L}(t)\right]\right\}$ (see Fig. 6.1). For tractability of the problem, let us consider that $y_{j}(0)=0 \forall j \in\{1, \ldots, L\}$, that is, all vehicles keep the same longitudinal position at the initial instant $t=0 \mathrm{~s}$; assume as well that longitudinal speeds are constant and fixed to $v_{I} \mathrm{~m} / \mathrm{s} \forall t \geq 0$. Given that at $t=0 \mathrm{~s}$ a total of $M+2$ one-dimensional obstacles are discovered $l$ meters ahead, vehicles only have a maximum

period of $t_{f}=\frac{l}{v_{I}}$ s from the initial time to execute an evasive maneuver to avoid or at least minimize the damage of a hypothetical accident. Thus, we wish to determine the trajectories' evolution of the $L$ vehicles during the time interval $\left[0, t_{f}\right]$ that can avoid the collision with the obstacles, with each other and against the lateral crash barriers, while optimizing some other mobility parameters established beforehand.

The constant longitudinal speed assumption might not be realistic in some circumstances, although it can be justified as a worst-case scenario where vehicles can only use the remaining lateral space when reaching the obstacles, without modifying the longitudinal speed. On the contrary, for low values of the maneuverability time interval $\left(t_{f}\right)$, and/or with high vehicle lengths, this assumption is better suited. In fact, by 


\section{COOPERATIVE COLLISION AVOIDANCE BY ACTIVE EVASIVE MANEUVERING}

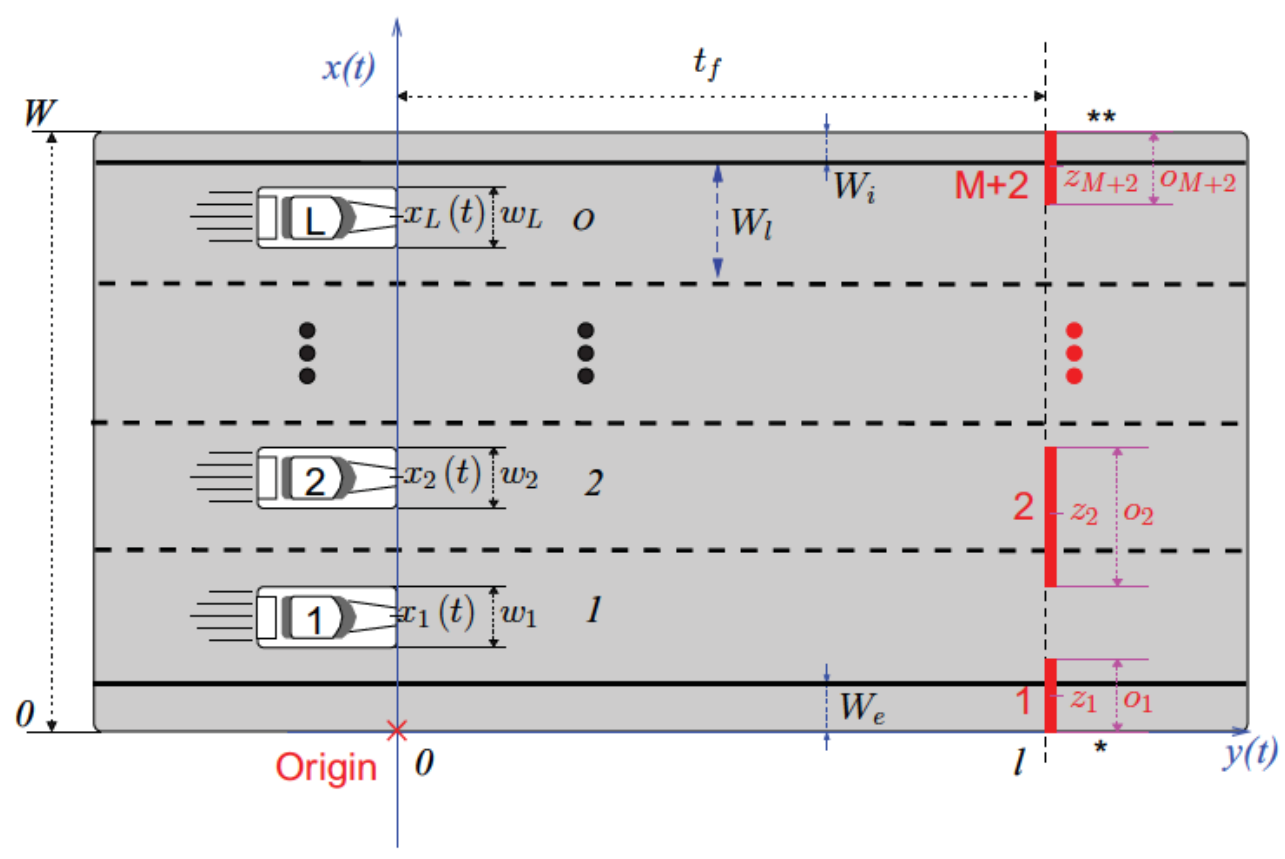

Figure 6.1: The general scenario under analysis

using low values of the maneuverability time interval $t_{f}$ we assume that at very short distances from the obstacles it is more reasonable to execute only lateral movements, at least as a first approach. In fact, sudden decelerations may become a higher risk for following vehicles (even more under higher values of the traffic density).

Given the assumptions presented above, we can restrict the problem to only governing the lateral mobility, and model the lateral motion of vehicles with the usual laws of kinematics as:

$$
\left\{\begin{array}{r}
\dot{x}_{j}(t)=v_{j}(t) \\
\dot{v}_{j}(t)=a_{j}(t)
\end{array} \quad \forall j \in\{1, \ldots, L\}\right.
$$

where $x_{j}(t) \in \mathbb{R}$ defines the instantaneous lateral position of vehicle $j$ according to axis $x, v_{j}(t) \in \mathbb{R}$ denotes the associated lateral speed of vehicle $j$, and $a_{j}(t) \in \mathbb{R}$ symbolizes the lateral acceleration of vehicle $j$. According to Eq. (6.1), the control domain variable that manages the mobility of each vehicle is the lateral acceleration $a_{j}(t)$.

Since our analysis reduces to the lateral motion of vehicles, we can perform a projection of the widths of vehicles and obstacles onto the $x$ axis as shown in Fig. 6.2 


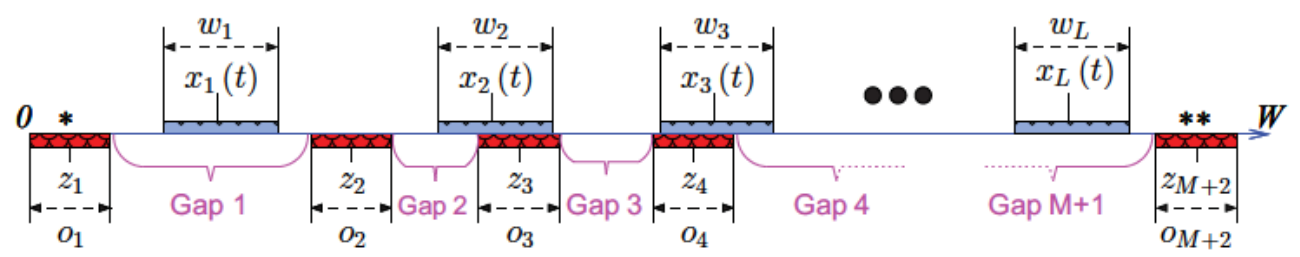

Figure 6.2: $x$-axis projections of elements

(x-axis projection), where $w_{j}$ corresponds to the width ${ }^{1}$ of vehicle $j$, while $z_{k}$ and $o_{k}$ refer to the central position and the width of obstacle $k$, respectively. The idea is to find an admissible control history ${ }^{2}$, in this case the lateral accelerations $a_{j}(t)$, which results in a set of admissible trajectories $x_{j}(t)$, which minimize a given performance measure or functional $J_{L, M}$ (see Section 6.3.2). We will also assume that: (1) vehicles can only move within the width limits of the road $[0, W]$ (see Fig. 6.1), whereas (2) the $x$-axis projections of vehicles cannot overlap on each other, which means that vehicle trajectories do not cross. Obstacles keep fixed and static, and (3) their projections can be overlapped by the vehicles' projections during the lateral motion. Additionally, the controls are subject to further constraints, that is, there is a maximum admissible lateral acceleration. The statements (1) to (3), as well as the constraints on the maximum allowable acceleration, are translated into state constraints as we will show. We consider the minimum number of obstacles to be $2(M=0)$, both located at the two extremes of the laterals of the road (crash barriers), symbols $\left(^{*}\right)$ and $(* *)$ respectively in Fig. 6.1). The projection on the $x$-axis of elements simplifies the original problem to a one-dimensional motion problem ${ }^{3}$, in which we only have to focus on the lateral displacement of the vehicles, thus notably simplifying the complexity of the set of possible solutions.

As we discuss later in Section 6.3.2 the functional we formulate is not differentiable so we propose a variation of the algorithm and solve a series of differentiable subproblems from which we will select the most suitable solution. In order to use the

\footnotetext{
${ }^{1}$ The $x$-axis projection of the width of a generic vehicle $j$ is assumed to be equal to $w_{j}$ regardless of the orientation of its associated trajectory, since the variability of the width's projection would increase the complexity of the problem in the constraining relations (nonlinearity of inequalities (6.10), see Subsection 6.3.1) and the expression of the performance measure. Moreover, since longitudinal speed $v_{I}$ is much higher than lateral speed $v_{j}(t)$, this variability can be neglected.

${ }^{2}$ That is, for all time instants.

${ }^{3}$ Only one spatial dimension, though the problem is still multidimensional in the sense that trajectories are computed for multiple vehicles.
} 


\section{COOPERATIVE COLLISION AVOIDANCE BY ACTIVE EVASIVE MANEUVERING}

MGP technique we first have to discretize the state equations. Afterwards we have to establish appropriate constraints which the state equations must obey. Finally, we propose a suitable performance measure or functional which is used to optimize the state trajectories. In the next subsections we describe these steps in detail.

\subsubsection{State equations and constraints}

Let us begin with the transformation of the state differential equations of (6.1) into discrete difference equations by using an incremental time period $\Delta t$ :

$$
\left\{\begin{aligned}
x_{j}(t+\Delta t) & \approx x_{j}(t)+v_{j}(t) \cdot \Delta t \\
v_{j}(t+\Delta t) & \approx v_{j}(t)+a_{j}(t) \cdot \Delta t
\end{aligned} \quad \forall j \in\{1, \ldots, L\}\right.
$$

Assuming we can observe the states at $0, \Delta t, 2 \Delta t, \ldots, N \Delta t$, with $N$ the number of steps in which the state trajectories are divided, and that the lateral acceleration can only be changed in these time instants, letting $t=k \Delta t$ and denoting the time step by just $k$ we have:

$$
\left\{\begin{array}{l}
x_{j}(k+1)=x_{j}(k)+v_{j}(k) \cdot \Delta t \\
v_{j}(k+1)=v_{j}(k)+a_{j}(k) \cdot \Delta t
\end{array} \quad \forall j \in\{1, \ldots, L\}\right.
$$

We express now the system as an evolution of the first time instant by using the acceleration as the independent variable. If we focus first on the lateral speed, we obtain:

$$
v_{j}(k+1)=V_{0 j}+\Delta t \cdot A_{0 j}+\Delta t \cdot \sum_{l=1}^{k} a_{j}(l)
$$

where $V_{0 j}$ denotes the initial lateral speed of vehicle $j$ and $A_{0 j}$ the initial lateral acceleration of vehicle $j$.

And for the expression of the lateral position we have:

$$
\begin{aligned}
x_{j}(k+1)= & X_{0 j}+(k+1) \Delta t \cdot V_{0 j}+ \\
& +(\Delta t)^{2} \cdot \sum_{l=0}^{k-1}(k-l) \cdot a_{j}(l)
\end{aligned}
$$

where $X_{0 j}$ is the initial lateral position of vehicle $j$. 
Substituting the right hand-side terms of the equations in the System (6.3) by taking the acceleration-dependent expressions for the position, Eq. (6.5), and speed, Eq. (6.4), we can now obtain the state trajectories $\left(x_{j}(k), v_{j}(k)\right.$ for $\left.k \in\{0, \ldots, N\}\right)$ by only calculating the controls (the lateral accelerations), with $a_{j}(k)$ defined for $k \in\{0, \ldots, N\}$. Although in reality it is sufficient to calculate the controls for the first $N$ instants (actually, the state trajectories are fully determined for $a_{j}(k)$ where $k \in\{0, \ldots, N-1\}$ ), we will always set the interval limit of integrals and summations to $k=N$ in order to keep uniformity in notation.

Since we are using a gradient descent-based approach, we need to set an initial guess for the controls $\left(a_{j}(k), k \in\{0, \ldots, N\}, j \in\{1, \ldots, L\}\right)$ to begin the descent:

$$
a_{j}^{(0)}(k)=A_{0 j} \quad k \in\{0, \ldots, N\}, j \in\{1, \ldots, L\}
$$

As boundary values for the position and speed of vehicle $j$ we have:

$$
\begin{aligned}
& x_{j}(0)=X_{0 j} \quad j \in\{1, \ldots, L\} \\
& v_{j}(0)=V_{0 j} \quad j \in\{1, \ldots, L\}
\end{aligned}
$$

From these initial configurations we obtain the solution that locally minimizes the performance measure (see Section 6.3.3). The minimization is local because the problem is non-convex, as we will see later.

The state variables and the controls must also adjust to the physical constraints of car motion. In this study we will introduce two main state restrictions, namely:

1. Lateral acceleration restrictions. The absolute value of the lateral acceleration cannot take a value higher than the limit $c\left(v_{I}\right) \mathrm{m} / \mathrm{s}^{2}$, where $v_{I}$ is the constant longitudinal speed of all vehicles and $c(\cdot): \mathbb{R} \rightarrow \mathbb{R}$ is a function of the longitudinal speed (same value for all vehicles).

$$
\left|a_{j}(t)\right| \leq c\left(v_{I}\right) \quad t \in\left[0, t_{f}\right], j \in\{1, \ldots, L\}
$$




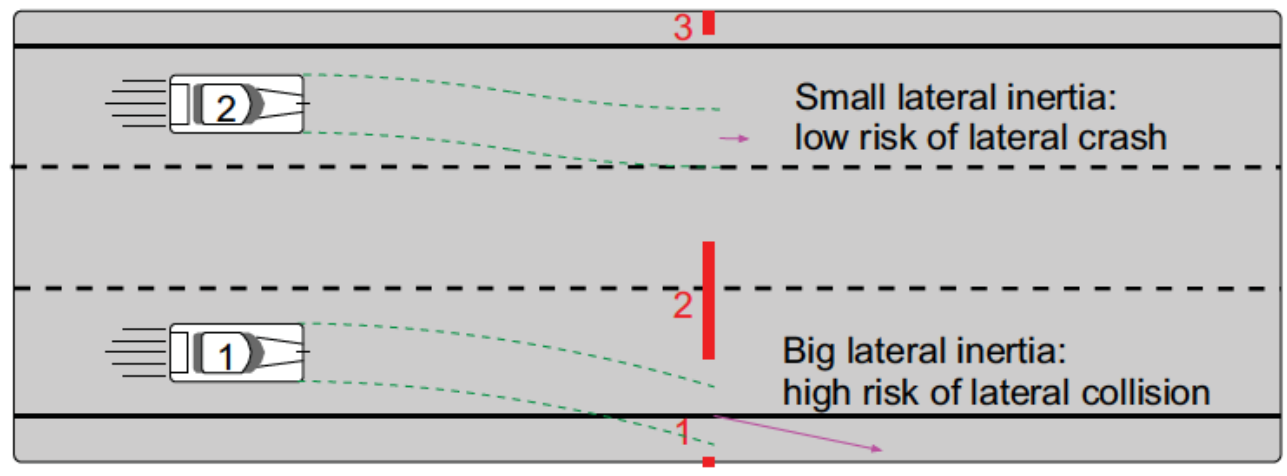

Figure 6.3: Interpretation of the Inertial Vector on graphs

2. Lateral position restrictions. The vehicles can only have a lateral displacement inside the width limits of the road, and their dimensions cannot overlap.

$$
\begin{gathered}
0<x_{1}(t) \pm \frac{w_{1}}{2}<\cdots<x_{L}(t) \pm \frac{w_{L}}{2}<W \\
t \in\left[0, t_{f}\right]
\end{gathered}
$$

Applying discretization for inequalities (6.9) and (6.10), we get:

$$
\begin{gathered}
-c\left(v_{I}\right) \leq a_{j}(k) \leq c\left(v_{I}\right) \\
0 \leq x_{1}(k) \pm \frac{w_{1}}{2} \leq \cdots \leq x_{L}(k) \pm \frac{w_{L}}{2} \leq W
\end{gathered}
$$

where

$$
k \in\{0, \ldots, N\}, j \in\{1, \ldots, L\}
$$

\subsubsection{Performance measure $\left(J_{L, M}\right)$}

In this section we focus on the statement and formulation of the general performance metric. The optimization goals we seek are summarized in the following three points:

1. Minimization of the lateral distance variance function at the end of the trajectory (at $t_{f}$ ). This term is used to maximize lateral distance at the time instant the obstacle/s is/are reached, $t_{f}$.

2. Minimization of the sum of the square of the lateral speeds at the end of the trajectory (at $t_{f}$ ). The purpose of this term is to reduce the lateral inertia (speed) at the time instant the obstacle/s is/are reached, $t_{f}$. 
When graphically representing vehicular trajectories (in Section 6.4), each vehicle shows a vector heading the same direction as that of the trajectory at the end of the path, with a modulus equal to the absolute value of the lateral speed at that time, which we call Inertial Vector from now on. Its purpose is to provide at a glance what is the inertial state of the vehicle at the end of the maneuver: the larger the modulus and the angle, the higher the lateral speed will be, thus resulting in an increased risk of further lateral collisions (see Fig. 6.3).

3. Minimization of the sum of the square of accelerations along the trajectory (during all the interval $\left.\left[0, t_{f}\right]\right)$. This term is used to reduce the negative impact on comfort of large accelerations along the trajectory.

These three optimization criteria are reflected in the dimensionless general performance measure $\left(J_{L, M}\right)$ of Eq. (6.13):

$$
J_{L, M}=\theta_{x} f_{x}+\theta_{v} f_{v}+\theta_{a} f_{a}
$$

where $f_{x}, f_{v}, f_{a}$ represent the functionals regarding the respective optimization goals presented earlier ${ }^{1}, L, M$ denotes the number of vehicles and intermediate obstacles (excluding the two crash barriers) in this evaluation, respectively. On the other hand, $\theta_{x}, \theta_{v}, \theta_{a} \in[0, \infty)$ are used to configure mathematically the weight of each addend on the general performance measure $J_{L, M^{2}}$. The general problem can be now formulated as:

$$
\left\{\begin{array}{cc}
\text { minimize } & J_{L, M} \\
\text { s.t. } & \text { constraints }
\end{array}\right.
$$

where constraints refer to the physical restrictions of car motion presented in Section 6.3.1. In order to characterize the problem statement in (6.14), we now focus on the definition of each of the $f_{x}, f_{v}$ and $f_{a}$ functionals.

\footnotetext{
${ }^{1} f_{x}$ being measured in $\mathrm{m}^{2}, f_{v}$ expressed in $(\mathrm{m} / \mathrm{s})^{2}$, and $f_{a}$ defined in $\left(\mathrm{m}^{2} / \mathrm{s}^{3}\right)$.

${ }^{2}$ To obtain a dimensionless performance measure $J_{L, M}, \theta_{x}$ is measured in $\mathrm{m}^{-2}, \theta_{v}$ is expressed in $(\mathrm{m} / \mathrm{s})^{-2}$, and $\theta_{a}$ also defined in $\left(\mathrm{m}^{-2} / \mathrm{s}^{-3}\right)$. In the rest of the text we disregard writing the corresponding units for the sake of simplicity (both for $f_{x}, f_{v}, f_{a}$ as well as for $\theta_{x}, \theta_{v}, \theta_{a}$ ). For obvious reason, this does not affect the optimization process.
} 


\section{COOPERATIVE COLLISION AVOIDANCE BY ACTIVE EVASIVE MANEUVERING}

\subsubsection{1 $f_{x}$ : minimization of the distance variance}

The main point in minimizing the distance variance functional $f_{x}$ is to displace vehicles laterally during the time interval $\left[0, t_{f}\right]$ so that each one can keep the largest distance to the surrounding elements on both sides. In order to express $f_{x}$, we introduce some definitions first:

Definition 1. Consider the two ordered sets

$$
S(t)=\left\{x_{1}(t), x_{2}(t), \ldots, x_{L}(t)\right\}
$$

and

$$
V=\left\{z_{1}, z_{2}, \ldots, z_{M+2}\right\}
$$

consisting of the instantaneous $x$-axis projections of the $L$ vehicles at time $t$, and the fixed positions on the projected axis of the $M+2$ obstacles, respectively.

We define $S(t) \cup V$ as the ordered union of the sets $S(t)$ and $V$ at time $t$, and let $\gamma_{i}(t)$ represent the $i$-th element of this union set, with $i \in\{1, \ldots, L+M+2\}$.

Let us note that the edges of the road will keep always the first and last position in the union set $S(t) \cup V$, that is:

$$
\begin{array}{r}
\gamma_{1}(t)=z_{1}, \quad \forall t \in\left[0, t_{f}\right], \\
\gamma_{L+M+2}(t)=z_{M+2}, \quad \forall t \in\left[0, t_{f}\right] .
\end{array}
$$

Definition 2. Given two elements $\gamma_{i}(t)$ and $\gamma_{j}(t)$ of the ordered union set $S(t) \cup V$, so that $\gamma_{i}(t) \leq \gamma_{j}(t)$, we define the function $\operatorname{dist}\left[\gamma_{i}(t), \gamma_{j}(t)\right]$ as the distance between the position on the $x$-axis of the right limit of the vehicle or obstacle $\gamma_{i}(t)$ and the left limit of $\gamma_{j}(t)$ (see Fig. 6.4 for an example).

Definition 3. Let $\tilde{\sigma}_{\text {dist }}^{2}(t)$ be a function that computes the variance in the distance between the lateral limits of consecutive elements on the projected axis. Then, considering the last two definitions, we express the distance variance function $\tilde{\sigma}_{\text {dist }}^{2}(t)$ as:

$$
\tilde{\sigma}_{\text {dist }}^{2}(t)=\sum_{i=1}^{M+L+1} \frac{\left(\operatorname{dist}\left[\gamma_{i}(t), \gamma_{i+1}(t)\right]\right)^{2}}{M+L}-\left(\frac{W-\mu}{M+L}\right)^{2}
$$

where $\mu=\sum_{j=1}^{L} w_{j}+\sum_{\beta=1}^{M} o_{\beta}$ refers to maximum distance occupied by the elements' projections on the axis when no one overlaps onto any other. Since the constants in 
Eq. (6.19) can be disregarded in the optimization procedure (their derivative does not influence the final solution), we will use the following expression of $\tilde{\sigma}_{\text {dist }}^{2}(t)$ instead:

$$
\sigma_{\text {dist }}^{2}(t)=\sum_{i=1}^{M+L+1}\left(\operatorname{dist}\left[\gamma_{i}(t), \gamma_{i+1}(t)\right]\right)^{2}
$$

The objective is then to minimize the adapted distance variance function $\sigma_{\text {dist }}^{2}(t)$ at the end of the trajectory, that is, at time instant $t_{f}$, Eq. (6.21), so that the lateral distances in the projected axis are maximized:

$$
f_{x}=\sigma_{d i s t}^{2}\left(t_{f}\right)
$$

In Fig. 6.4 an example for the case $L=1, M=1$ is shown. In that case, a single vehicle can choose the left or right gap, marked as cases A) and B). It can be seen how $f_{x}$ is a piecewise function whose arguments depend on the instantaneous position of each vehicle with respect to the obstacles. It makes this function non-differentiable, as we discuss at the end of this section.

\subsubsection{2 $f_{v}$ : minimization of the final lateral speed}

The main goal of this subfunctional is to minimize the final lateral speed. Its expression is:

$$
f_{v}=\sum_{j=1}^{L} v_{j}^{2}\left(t_{f}\right)
$$

\subsubsection{3 $f_{a}$ : minimization of the instantaneous lateral acceleration}

In this case, $f_{a}$ is used to minimize the total acceleration along the trajectory to execute the evasive maneuver. Eq. (6.23) defines this subfunctional:

$$
f_{a}=\sum_{j=1}^{L} \int_{0}^{t_{f}} a_{j}^{2}(t) d t
$$

The general performance measure then becomes: 


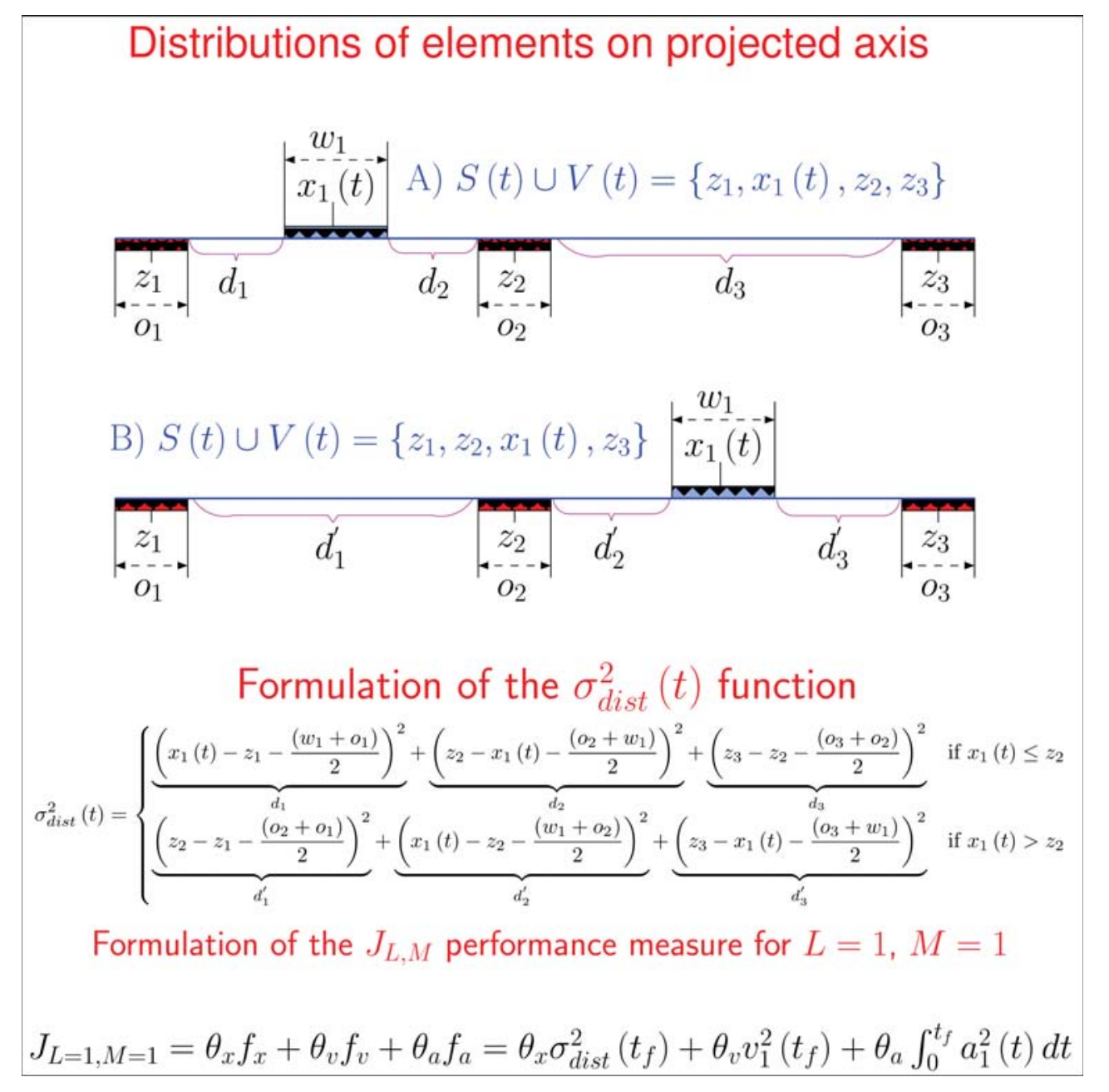

Figure 6.4: Example of $J_{L, M}$ formulation for $L=1, M=1(L=1$ vehicle, $M+2=3$ obstacles)

$$
\begin{aligned}
J_{L, M} & =\theta_{x}\left[\sum_{i=1}^{M+L+1}\left(\operatorname{dist}\left[\gamma_{i}\left(t_{f}\right), \gamma_{i+1}\left(t_{f}\right)\right]\right)^{2}\right]+ \\
& +\theta_{v}\left[\sum_{j=1}^{L} v_{j}^{2}\left(t_{f}\right)\right]+ \\
& +\theta_{a}\left[\sum_{j=1}^{L} \int_{0}^{t_{f}} a_{j}^{2}(t) d t\right] .
\end{aligned}
$$

Once the general expression for the performance measure is specified, now it is turn 
to obtain the associated gradient function that will be used to minimize the general performance measure. For this purpose, the first action to do is to discretize the performance measure in order to express it as a function of the $L \times(N+1)$ variables: the controls $a_{j}(k)$, where $j \in\{1, \ldots, L\}, k \in\{0, \ldots, N\}$. Next we obtain the corresponding gradient function by deriving respect to each of the $L \times N$ control variables, whose values are calculated to match our optimization criteria. Then we substitute this array of controls in the discretized difference equations of (6.3) to finally obtain the desired trajectories the vehicles have to follow.

Discretizing $f_{v}$ and $f_{a}$ and expressing the result as a function of the controls is straightforward. Furthermore, in this case, both $f_{v}$ and $f_{a}$ are differentiable functions for which the Gradient Projection algorithm can be used in order to obtain the minimum we seek as a solution. However, the expression for $f_{x}=\sigma_{\text {dist }}^{2}\left(t_{f}\right)$ has to be formulated as a piecewise function whose arguments depend on the instantaneous position of each vehicle respect to the obstacles (see Fig. 6.4). The number of subfunctions of the piecewise functional $f_{x}$ is the number of possible compositions of a group of $L$ elements (vehicles) into $M+1$ parts (gaps). It has a total of $R_{L, M+1}=C(M+L, M)$ solutions ${ }^{1}$. This solution is called a weak composition of $L$ into $(M+1)$ parts, or a weak $(M+1)$ composition of $L$ (see (102)).

Thus, $R_{L, M+1}$ is the total number of subfunctions that build the corresponding $f_{x}=\sigma_{\text {dist }}^{2}\left(t_{f}\right)$ piecewise functional. The non-differentiability of general piecewise functions, and in particular, the $f_{x}=\sigma_{\text {dist }}^{2}\left(t_{f}\right)$ functional, implies that the performance measure $J_{L, M}$ is also non-differentiable Thus, a gradient descent-based approach cannot be directly applied to solve the optimization problem (6.14) when formulated this way.

To overcome this issue, we will reformulate the non-differentiable optimization problem (6.14) as a set of differentiable optimization subproblems from which we will choose the solution which better fits our requirements.

\subsubsection{Reformulation as a set of differentiable subproblems}

It is possible to overcome the non-differentiability of the problem by reformulating it as a series of differentiable subproblems, everyone of which has to be minimized, and then choose the solution with the lowest cost. In plain words, the overall idea is to

\footnotetext{
${ }^{1} C(M+L, M)$ denotes the combinatorial number $\left(\begin{array}{c}M+L \\ M\end{array}\right)$ (see (102)).
} 


\section{COOPERATIVE COLLISION AVOIDANCE BY ACTIVE EVASIVE MANEUVERING}

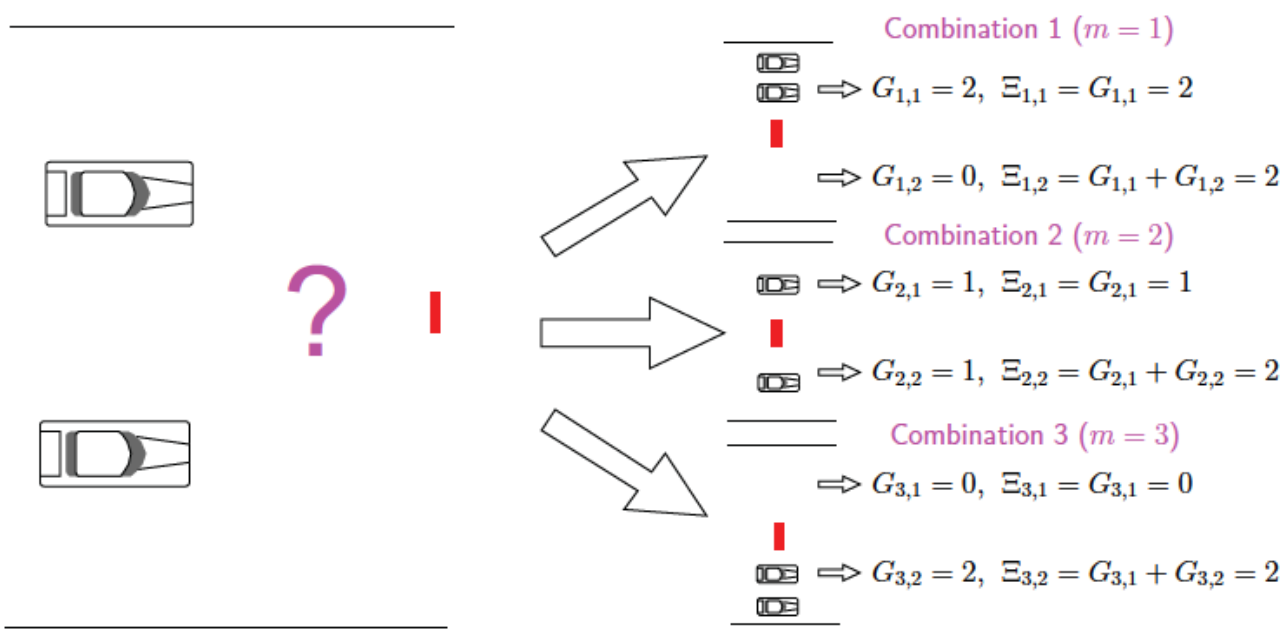

Figure 6.5: Exemplification of combination reordering for $M=1, L=2$. Each alternative is a weak $(M+1)$-composition of $L$

evaluate the functional of every possible combination of vehicles into gaps and select the combination with the lowest value.

More formally, let us describe again the procedure to establish each subfunctional: when a total of $L$ vehicles is approaching $M+2$ obstacles, each vehicle has at most $M+1$ gaps to pass through. Since vehicles cannot overlap each other laterally, the number of possible reorderings is reduced, having a total of $R_{L, M+1}$ possible reordering combinations.

Definition 4. Let $G_{m, \beta}^{(L, M)}$ denote the number of vehicles willing to pass through a certain gap $\beta \in\{1, \ldots, M+1\}$ in the combination $m \in\left\{1, \ldots, R_{L, M+1}\right\}$. These numbers $G_{m, \beta}^{(L, M)}$ are obtained by solving the system:

$$
\begin{aligned}
& \sum_{\beta=1}^{M+1} G_{m, \beta}^{(L, M)}=L, \\
& 0 \leq G_{m, \beta}^{(L, M)} \leq L,
\end{aligned}
$$

(see (102) and have a look at Fig. 6.5 to understand this concept by a given example).

It is easy to see that the number of solutions to this nonnegative-integer-sum equality is the same as the number of subfunctions of the corresponding non-convex, nondifferentiable function $\sigma_{\text {dist }}^{2}$ (see Subsection 6.3.2). 


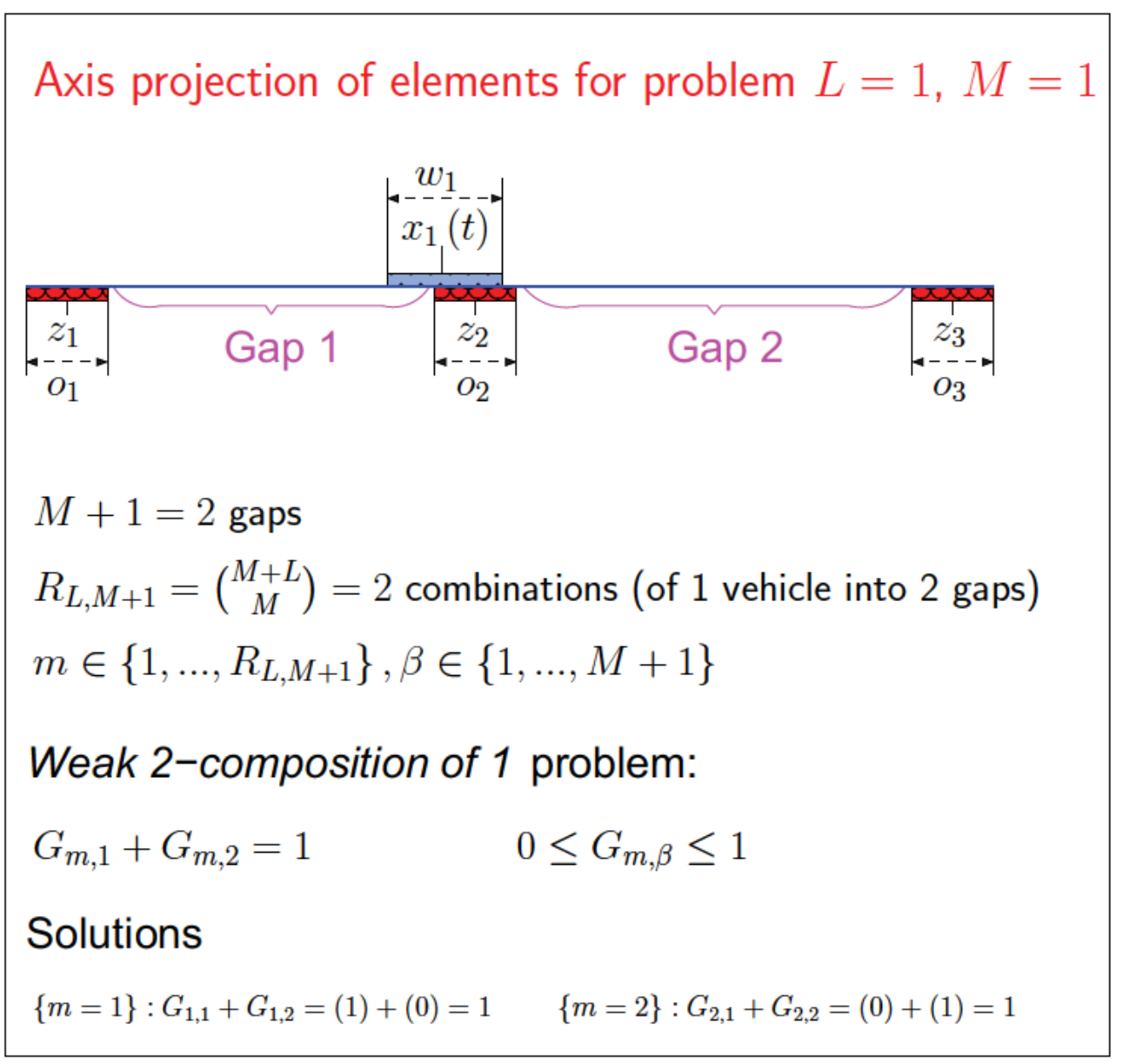

Figure 6.6: Weak (M+1)-composition of $L$ problem, $L$ vehicles into $M+1$ gaps $(L=1$ vehicle, $M+2=3$ obstacles)

Let $\Xi_{m, l}^{(L, M)}=\sum_{\beta=1}^{l} G_{m, \beta}^{(L, M)}$ denote the cumulative sum of vehicles until gap $\beta$. For notation easiness, superindex ${ }^{(L, M)}$ will be omitted in the following equations, assuming that these parameters, namely $L$ and $M$, are previously established and known. We assume that vehicles maintain their order in the $x$-axis, so that, the indexes of the $G_{m, \beta}$ vehicles heading for gap $\beta$ in the combination $m$ will be $\left\{\Xi_{m, \beta-1}+1, \ldots, \Xi_{m, \beta}\right\}$.

As we said, the general idea is to quantify mathematically how good is for the $G_{m, \beta}$ vehicles leading to a certain gap $\beta$ to pass through it, and do the same for the rest of the gaps of this combination $m$. This evaluation is accounted for with the $\phi_{m, \beta}(t)$ function. Then, for a combination $m$, the results of the $\phi_{m, \beta}(t)$ function for the $M+1$ gaps 


\section{COOPERATIVE COLLISION AVOIDANCE BY ACTIVE EVASIVE MANEUVERING}

$(\beta \in\{1, \ldots, M+1\})$ are summed, to obtain the distance variance of vehicles heading for this gap $\left(\sigma_{m, d i s t}^{2}\right)$ in combination $m$. Adding the other two performance criteria $f_{v}$ and $f_{a}$ to the $\sigma_{m, d i s t}^{2}$ function of combination $m$ will give us the performance cost functional of vehicles choosing to pass through obstacles as defined by the ordering scheme of the present combination $m$. Doing this for the rest of the $R_{L, M+1}-1$ combinations, we will obtain the set of performance cost functionals $\left\{J_{L, M}^{(1)}, \ldots, J_{L, M}^{\left(R_{L, M+1}\right)}\right\}$ regarding all combinations. We proceed to minimize each $J_{L, M}^{(q)}$ subfunctional to obtain the new set $\left\{\overline{J_{L, M}^{(1)}}, \ldots, \overline{J_{L, M}^{\left(R_{L, M+1}\right)}}\right\}$, from which we will choose the entry with the lowest value: $\overline{J_{L, M}^{(q)}}$.

Let us now express the previous concepts more formally. For each $m \in\left\{1, \ldots, R_{L, M+1}\right\}$ we solve the following non-convex differentiable subproblem:

$$
\left\{\begin{array}{cc}
\operatorname{minimize} & J_{L, M}(m) \\
\text { s.t. } & \text { constraints }
\end{array}\right.
$$

by using the MGP procedure (see Subsections 6.3.4 and 6.3.5). The result is a locally optimal value of the functional and its associated control history, namely $\overline{J_{L, M}^{(m)}}$ and $\overline{a_{j}^{(m)}}(k), k \in\{0, \ldots, N\}$, respectively.

Then, to find the optimum value of the general problem (6.14) by applying the new approach, we have to choose the combination $q \in\left\{1, \ldots, R_{L, M+1}\right\}$ with the lowest performance measure, that is

$$
\overline{J_{L, M}}=\min \left\{\overline{J_{L, M}^{(1)}}, \ldots, \overline{J_{L, M}^{\left(R_{L, M+1}\right)}}\right\}=\overline{J_{L, M}^{(q)}} .
$$

Therefore, the control history values that globally minimize the functional $J_{L, M}$ are those associated with this combination $q$, that is

$$
\overline{a_{j}}(k)=\overline{a_{j}^{(q)}}(k), k \in\{0, \ldots, N-1\} .
$$

The $J_{L, M}^{(m)}$ functions take the expression:

$$
\begin{aligned}
J_{L, M}^{(m)} & =\theta_{x} f_{m, x}+\theta_{v} f_{v}+\theta_{a} f_{a}= \\
& =\theta_{x}\left[\sigma_{m, \text { dist }}^{2}\left(t_{f}\right)\right]+\theta_{v}\left[\sum_{j=1}^{L} v_{j}^{2}\left(t_{f}\right)\right]+ \\
& +\theta_{a}\left[\sum_{j=1}^{L} \int_{0}^{t_{f}} a_{j}^{2}(t) d t\right] .
\end{aligned}
$$




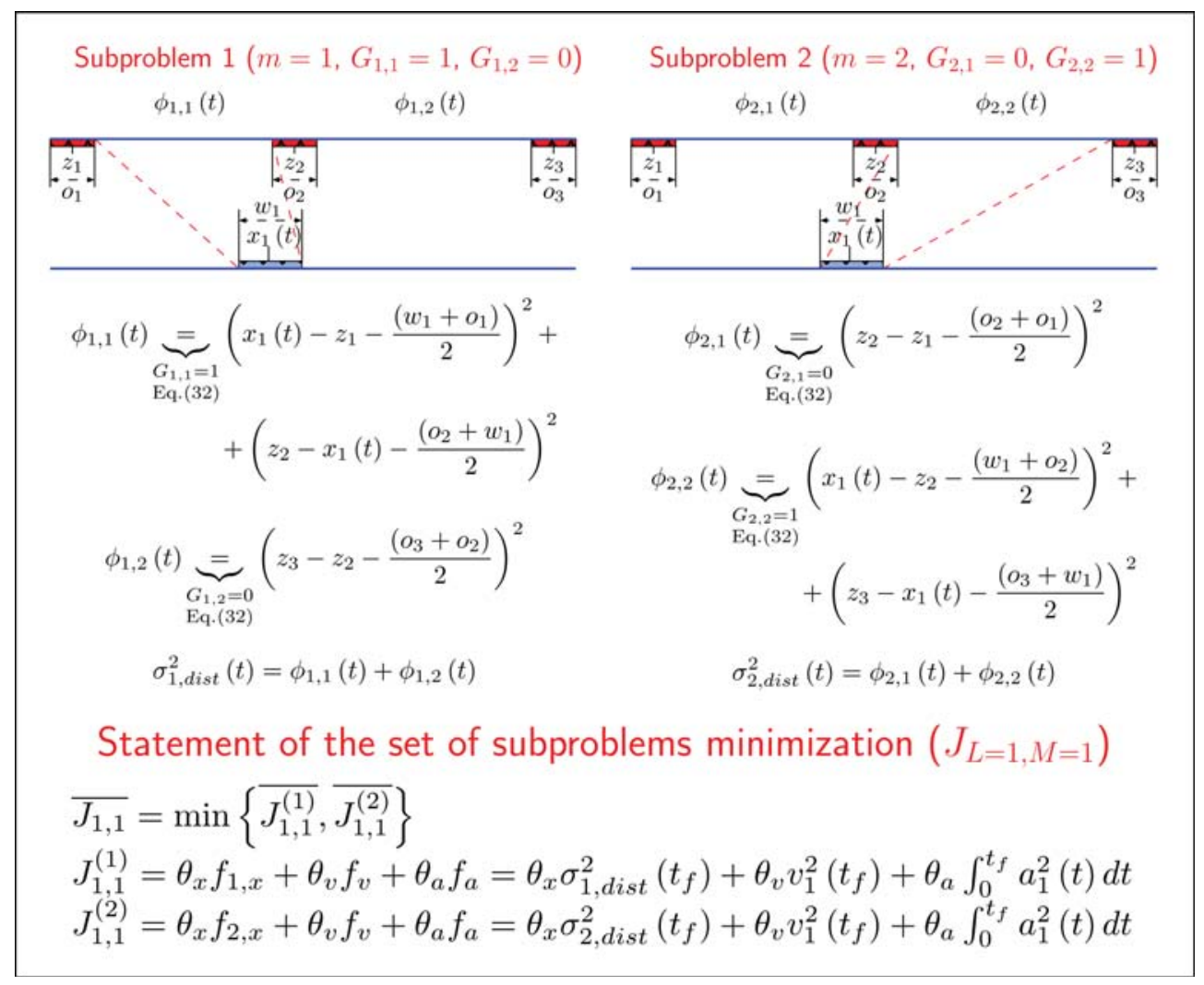

Figure 6.7: Relation of subproblems associated to $J_{L, M}$ for $L=1, M=1$ ( $L=1$ vehicle, $M+2=3$ obstacles)

where in this case $f_{m, x}=\sigma_{m, d i s t}^{2}\left(t_{f}\right)$ represents the adapted distance variance functional, as in Eq. (6.20), for each $J_{L, M}^{(m)}$ minimization subproblem in the new formulation proposal.

Bearing in mind the previous discussion, we express $\sigma_{m, d i s t}^{2}(t)$ as:

$$
\sigma_{m, d i s t}^{2}(t)=\sum_{\beta=1}^{M+1} \phi_{m, \beta}(t)
$$

with 


\section{COOPERATIVE COLLISION AVOIDANCE BY ACTIVE EVASIVE MANEUVERING}

$$
\begin{aligned}
\phi_{m, \beta}(t) & =\left(z_{\beta+1}-z_{\beta}-C_{1}\right)^{2}+ \\
& +G_{m, \beta}\left[\left(x_{\Xi_{m, \beta-1}+1}(t)-z_{\beta}-C_{2}\right)^{2}+\right. \\
& +\left(z_{\beta+1}-x_{\Xi_{m, \beta}}(t)-C_{3}\right)^{2}- \\
& \left.-\frac{\left.\left(z_{\beta+1}-z_{\beta}-C_{1}\right)^{2}\right]+}{G_{m, \beta}}\right] \\
& +\sum_{l=\Xi_{m, \beta-1}+1}^{\Xi_{m, \beta}-1}\left[\left(x_{l+1}(t)-x_{l}(t)-C_{4}(l+1)\right)^{2}-\right. \\
& -\left(x_{\Xi_{m, \beta-1}+1}(t)-z_{\beta}-C_{2}\right)^{2}- \\
& \left.-\left(z_{\beta+1}-x_{\Xi_{m, \beta}}(t)-C_{3}\right)^{2}\right]
\end{aligned}
$$

where we have:

$$
\begin{array}{cc}
C_{1}=\frac{o_{\beta+1}+o_{\beta}}{2} & C_{2}=\frac{w_{\Xi_{m, \beta-1}+1}+o_{\beta}}{2} \\
C_{3}=\frac{o_{\beta+1}+w_{\Xi_{m, \beta}}}{2} & C_{4}(l)=\frac{\left(w_{l}+w_{l-1}\right)}{2} \\
G_{m, 0}=0 & G_{m, M+2}=0
\end{array}
$$

We see that the $\phi_{m, \beta}(t)$ function computes the adapted distance variance for the $G_{m, \beta}$ vehicles present in the $\beta$-th gap of the $m$-th combination (an example is provided in Fig. 6.8). Finally, Eq. (6.31) calculates the sum of the local distance variances of the $M+1$ gaps, giving the value of the total adapted distance variance for the combination $m$ (see Figs. 6.6 and 6.7 on how to obtain each of the $J_{L, M}^{(m)}$ functions for the same case of Fig. 6.4).

The following step is to obtain the acceleration history for the $L$ vehicles which minimizes the $J_{L, M}^{(m)}$ function for each instance of the $R_{L, M+1}$ possible combinations, and then choose the combination (input of accelerations) which globally minimizes the problem (6.14). For this purpose we have to apply discretization and derive respect to $a_{j}(k)(1 \leq j \leq L, 0 \leq k \leq N)$. The discretized version of the $J_{L, M}^{(m)}$ function takes the form: 


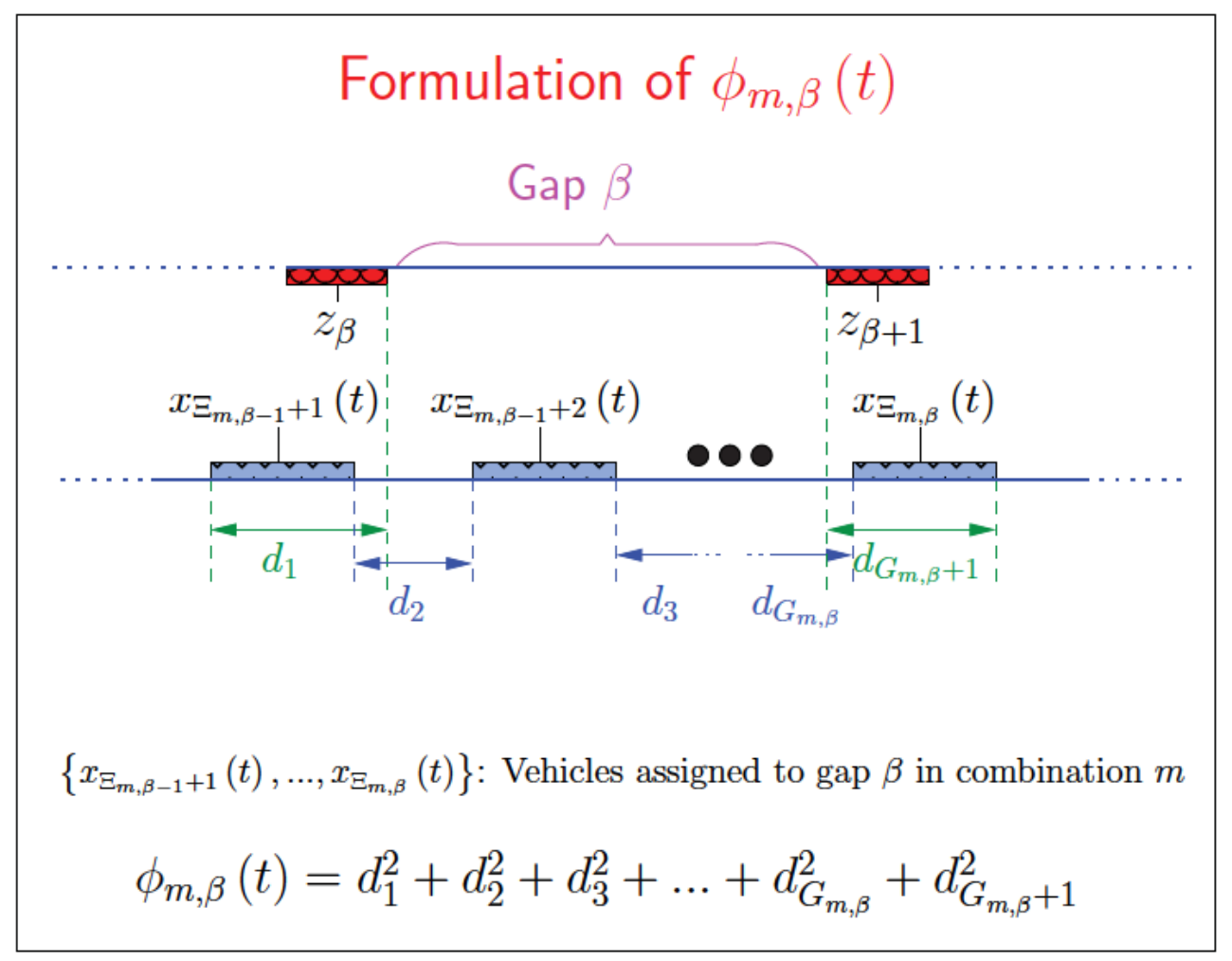

Figure 6.8: Graphic example of how to calculate the $\phi_{m, \beta}(t)$ function

$$
\begin{aligned}
J_{L, M}^{(m)} & =\theta_{x}\left[\sigma_{m, d i s t}^{2}(N)\right]+\theta_{v}\left[\sum_{j=1}^{L} v_{j}^{2}(N)\right]+ \\
& +\theta_{a}\left[\Delta t \sum_{j=1}^{L} \sum_{k=0}^{N} a_{j}^{2}(k)\right] .
\end{aligned}
$$

Deriving $J_{L, M}^{(m)}$ with respect to a fixed $a_{p}(s)$, we obtain:

$$
\begin{aligned}
\frac{\partial J_{L, M}^{(m)}}{\partial a_{p}(s)} & =\theta_{x}\left(\frac{\partial \sigma_{m, d i s t}^{2}(N)}{\partial a_{p}(s)}\right)+ \\
& +\theta_{v} \alpha_{v}(p, s)+ \\
& +\theta_{a} \alpha_{a}(p, s)
\end{aligned}
$$




\section{COOPERATIVE COLLISION AVOIDANCE BY ACTIVE EVASIVE MANEUVERING}

with

$$
\begin{aligned}
& \alpha_{v}(n, l)=\left\{\begin{array}{cc}
0 & n \neq p \\
2 \Delta t\left[V_{0 p}+\Delta t A_{0 p}+\right. & \\
\left.+\Delta t \sum_{k=1}^{N-1} a_{p}(k)\right] & n=p
\end{array}\right. \\
& \alpha_{a}(n, l)=\left\{\begin{array}{cc}
0 & n \neq p \vee l \neq s \\
2 \Delta t a_{p}(s) & n=p \wedge l=s
\end{array}\right.
\end{aligned}
$$

If we focus now on the term in $(6.36)$ containing $\sigma_{m, d i s t}^{2}$, we can define $\frac{\partial \sigma_{m, d i s t}^{2}(k)}{\partial a_{p}(s)}$ as:

$$
\frac{\partial \sigma_{m, d i s t}^{2}(k)}{\partial a_{p}(s)}=\left\{\begin{array}{cc}
\frac{\partial \phi_{m, \beta}(k)}{\partial a_{p}(s)} & \Xi_{m, \beta-1}+1 \leq p \leq \Xi_{m, \beta} \\
0 & \Xi_{m, \beta-1}+1>p \vee \Xi_{m, \beta}<p
\end{array}\right.
$$

The condition in (6.39) for $\frac{\partial \sigma_{m, d i s t}^{2}(k)}{\partial a_{p}(s)}=0$ is obvious (the derivative is not null only for those cases in which the vehicle $p$ is inside gap $\beta$ ), so we will focus only on the case where $\Xi_{m, \beta-1}+1 \leq p \leq \Xi_{m, \beta}$. From this it is easy to obtain:

$$
\begin{aligned}
& \frac{\partial \phi_{m, \beta}(k)}{\partial a_{p}(s)}= \\
& =G_{m, \beta}\left[2\left(x_{\Xi_{m, \beta-1}+1}(k)-z_{\beta}-C_{2}\right) \frac{\partial x_{\Xi_{m, \beta-1}+1}(k)}{\partial a_{p}(s)}-\right. \\
& \left.-2\left(z_{\beta+1}-x_{\Xi_{m, \beta}}(k)-C_{3}\right) \frac{\partial x_{\Xi_{m, \beta}}(k)}{\partial a_{p}(s)}\right]+ \\
& +\sum_{m=\Xi_{m, \beta-1}+1}^{\Xi_{m, \beta}-1}\left[2\left(x_{m+1}(k)-x_{m}(k)-C_{4}(m+1)\right) \cdot\right. \\
& \cdot\left(\frac{\partial x_{m+1}(k)}{\partial a_{p}(s)}-\frac{\partial x_{m}(k)}{\partial a_{p}(s)}\right)- \\
& -2\left(x_{\Xi_{m, \beta-1}+1}(k)-z_{\beta}-C_{2}\right) \frac{\partial x_{\Xi_{m, \beta-1}+1}(k)}{\partial a_{p}(s)}+ \\
& +2\left(z_{\beta+1}-x_{\Xi_{m, \beta}}(k)-C_{3}\right) \cdot \\
& \left.\cdot \frac{\partial x_{\Xi_{m, \beta}}(k)}{\partial a_{p}(s)}\right]
\end{aligned}
$$


where

$$
\frac{\partial x_{j}(k)}{\partial a_{p}(s)}=\left\{\begin{array}{cc}
0 & (p \neq j) \vee(k<s+2) \\
(\Delta t)^{2}(k-s-1) & (p=j) \wedge(k \geq s+2)
\end{array}\right.
$$

It is possible to particularize Eq. (6.40) further depending on the surrounding elements of the derivation index $p$, by abstracting position, speed, acceleration and width for vehicles and obstacles as seen in Eq. (6.42) (see Appendix C for details on operations):

$$
\begin{aligned}
& \frac{\partial \phi_{m, \beta}(k)}{\partial a_{p}(s)}= \\
& =2(\Delta t)^{2}(k-s-1)\left[\left(2 \pi_{0 p}-\pi_{0 p+1}-\pi_{0 p-1}\right)+\right. \\
& +k \Delta t\left(2 \lambda_{0 p}-\lambda_{0 p+1}-\lambda_{0 p-1}\right)+ \\
& +(\Delta t)^{2} \sum_{l=0}^{k-2}(k-l-1)\left(2 \psi_{p}(l)-\psi_{p+1}(l)-\psi_{p-1}(l)\right)+ \\
& \left.+\frac{\varpi_{p+1}-\varpi_{p-1}}{2}\right]
\end{aligned}
$$

where

$$
\left.\begin{array}{c}
\psi_{n}(l)=0 \\
\lambda_{0 n}=0 \\
\pi_{0 n}=z_{\beta-1} \\
\varpi_{n}=o_{\beta-1}
\end{array}\right\} n \leq \Xi_{m, \beta-1}
$$

From Eq. (6.42) and its specification in (6.43) we can notice the influence on the mobility of vehicle $p$ (term $\psi_{p}$ ) originated by its immediate neighbors (terms $\psi_{p+1}$ and $\psi_{p-1}$ ), depending on the class of element (either if both are obstacles, one vehicle and one obstacle, or two vehicles). Intuitively, this term of the performance measure reflects the distance variance minimization objective we are looking for. 


\section{COOPERATIVE COLLISION AVOIDANCE BY ACTIVE EVASIVE MANEUVERING}

\subsubsection{The Modified Gradient Projection (MGP) algorithm}

Despite overcoming non-differentiability, each subfunctional $J_{L, M}^{(q)}$ is still non-convex. The reason is that, since we are optimizing respect to the final lateral position of vehicles (at time instant $t_{f}$ ), intermediate trajectories are multiple (see (99)). As we previously introduced, to overcome this we use a modified version of the Gradient Projection algorithm, the Modified Gradient Projection (MGP), whose steps are summarized next:

1. Set an initial guess for the controls $\left(a_{j}(k), k \in\{0, \ldots, N\}\right)$, (see Subsection 6.3.1).

2. Perform the gradient descent by the typical approach $\left(x_{n+1}=x_{n}-\alpha \cdot \nabla f\left(x_{n}\right)\right.$, see $(103))$.

(a) If $\nabla f\left(x_{n}\right)=0$, go to Step 4 .

(b) If $\nabla f\left(x_{n}\right) \neq 0$ and no constraints (section 6.3.1) are violated, continue with Step 2.

(c) If $\nabla f\left(x_{n}\right) \neq 0$ and one or more constraints (section 6.3.1) are violated, go to Step 3.

3. Move along the projection of the negative gradient onto the boundary of the admissible region of controls (based on the Gradient Projection approach for constrained convex minimization, see $(19,86))$.

(a) If a minimum is found (between the values on the boundaries), go to Step 4.

(b) Else, continue to Step 3.

4. $x_{n}$ is the local solution of the problem. Finalize.

The Gradient Projection algorithm initially projects the negative gradient direction (if minimizing) onto the closest boundary of the admissible region of values, and then it continues 'descending' along the boundaries until the minimum of the admissible region is found. This initial projection is valid if the function is convex, but in our case it is not, thus requiring to skip this initial operation to avoid getting trapped into multiple non-convex regions. With MGP we will only descend along the limits of the region of possible values if, while locally optimizing, we reach a boundary of the admissible region. 


\subsubsection{Summary of optimization strategy}

In this subsection we briefly summarize the different steps followed to solve the Optimal Control Problem with MGP (Section 6.3.4). This procedure is based on the work of (19), accounting for some modifications to include the proposed MGP procedure:

1. Formulation of the differential equations which model the behavior of the system, and its expression, System (6.1), as a system of difference equations. Determination of the expressions for the state constraints, Eqs. (6.9-6.10), and formulation of the general performance measure to set up the optimization requirements (6.13$6.24)$.

2. Determination of the expressions of the state constraints, Eqs. (6.11), in terms of the control domain (accelerations history). Reformulation of the minimization problem as a set of differentiable subproblems, Eqs. (6.27-6.28), and expression of the subfunctionals in terms of the control domain history (Subsection 6.3.3).

3. Input of an initial control history (accelerations, see Section 6.3.1) and calculation of the associated state trajectories (initial positions and speeds for $k \in\{0, \ldots, N\}$ ).

4. Local minimization of the $J_{L, M}^{(m)}$ subfunctionals by using the MGP algorithm (see Section 6.3.4 for details on its execution).

5. Selection of the combination $J_{L, M}^{(q)}$ achieving the lowest value of the general performance measure $J_{L, M}$. Substitute values for $a_{j}^{(q)}(k)$ in the System of difference Eqs. (6.3) by using Eqs. (6.4) and (6.5). Calculation of the optimum state trajectories for the $L$ vehicles.

\subsection{Evaluation results}

As was said previously, our goal in this work is to introduce a relatively simple approach to obtain optimum cooperative maneuvers when facing collision avoidance at high speeds. Therefore, the main aim of this section is to provide results which show the validity of the proposed procedure. Additionally, we are interested in evaluating the influence of the different components of the functional on the resulting trajectories and the potential flexibility to shape them to our needs. A more in-depth study of particular possible scenarios as well as the most relevant cases are left as future work. 


\section{COOPERATIVE COLLISION AVOIDANCE BY ACTIVE EVASIVE MANEUVERING}

Table 6.1: Configuration parameters for the evaluation cases

\begin{tabular}{|c|c|c|c|}
\hline Parameter & Evaluation 1 & Evaluation 2 & Evaluation 3 \\
\hline$N$ & 100 & 20 & 20 \\
\hline$L$ & 2 & 2 & 2 \\
\hline$M$ & 1 & 1 & 1 \\
\hline$O$ & 3 & 3 & 3 \\
\hline$t_{f}$ & $\{0.9,1,1.1\} \mathrm{s}$ & $\{0.9,1,1.1\} \mathrm{s}$ & $1.2 \mathrm{~s}$ \\
\hline$v_{I}$ & $33 \mathrm{~m} / \mathrm{s}$ & $33 \mathrm{~m} / \mathrm{s}$ & $33 \mathrm{~m} / \mathrm{s}$ \\
\hline$c\left(v_{I}\right)$ & $5.5432 \mathrm{~m} / \mathrm{s}^{2}$ & $5.5432 \mathrm{~m} / \mathrm{s}^{2}$ & $5.5432 \mathrm{~m} / \mathrm{s}^{2}$ \\
\hline$W_{l}$ & $3.5 \mathrm{~m}$ & $3.5 \mathrm{~m}$ & $3.5 \mathrm{~m}$ \\
\hline$W_{i}$ & $1.5 \mathrm{~m}$ & $1.5 \mathrm{~m}$ & $1.5 \mathrm{~m}$ \\
\hline$W_{e}$ & $2.5 \mathrm{~m}$ & $2.5 \mathrm{~m}$ & $2.5 \mathrm{~m}$ \\
\hline$W$ & $14.5 \mathrm{~m}$ & $14.5 \mathrm{~m}$ & $14.5 \mathrm{~m}$ \\
\hline$X_{0 j}$ & $\{4.25,11.25\} \mathrm{m}$ & $\{4.25,11.25\} \mathrm{m}$ & $\{4.25,11.25\} \mathrm{m}$ \\
\hline$w_{j}$ & $2 \mathrm{~m}$ & $2 \mathrm{~m}$ & $2 \mathrm{~m}$ \\
\hline$V_{0 j}$ & $0 \mathrm{~m} / \mathrm{s}$ & $0 \mathrm{~m} / \mathrm{s}$ & $0 \mathrm{~m} / \mathrm{s}$ \\
\hline$A_{0 j}$ & $0 \mathrm{~m} / \mathrm{s}^{2}$ & $0 \mathrm{~m} / \mathrm{s}^{2}$ & $0 \mathrm{~m} / \mathrm{s}^{2}$ \\
\hline$a_{j}^{0}(k)$ & $0 \mathrm{~m} / \mathrm{s}^{2}$ & $0 \mathrm{~m} / \mathrm{s}^{2}$ & $0 \mathrm{~m} / \mathrm{s}^{2}$ \\
\hline$z_{\beta}$ & $\{0,4.5,14\} \mathrm{m}$ & $\{0,4.5,14\} \mathrm{m}$ & $\{0,4.5,14\} \mathrm{m}$ \\
\hline$o_{\beta}$ & $\{0,2,1\} \mathrm{m}$ & $\{0,2,1\} \mathrm{m}$ & $\{0,2,1\} \mathrm{m}$ \\
\hline$\theta_{x}$ & $1 \mathrm{~m}^{-2}$ & $1 \mathrm{~m}^{-2}$ & $1 \mathrm{~m}^{-2}$ \\
\hline$\theta_{v}$ & $0(\mathrm{~m} / \mathrm{s})^{-2}$ & $\{100,10,1,0.1\}(\mathrm{m} / \mathrm{s})^{-2}$ & $\{10,1,0.5\}(\mathrm{m} / \mathrm{s})^{-2}$ \\
\hline$\theta_{a}$ & $0\left(\mathrm{~m}^{-2} / \mathrm{s}^{-3}\right)$ & $0\left(\mathrm{~m}^{-2} / \mathrm{s}^{-3}\right)$ & $\{0,0.1,0.5\}\left(\mathrm{m}^{-2} / \mathrm{s}^{-3}\right)$ \\
\hline
\end{tabular}

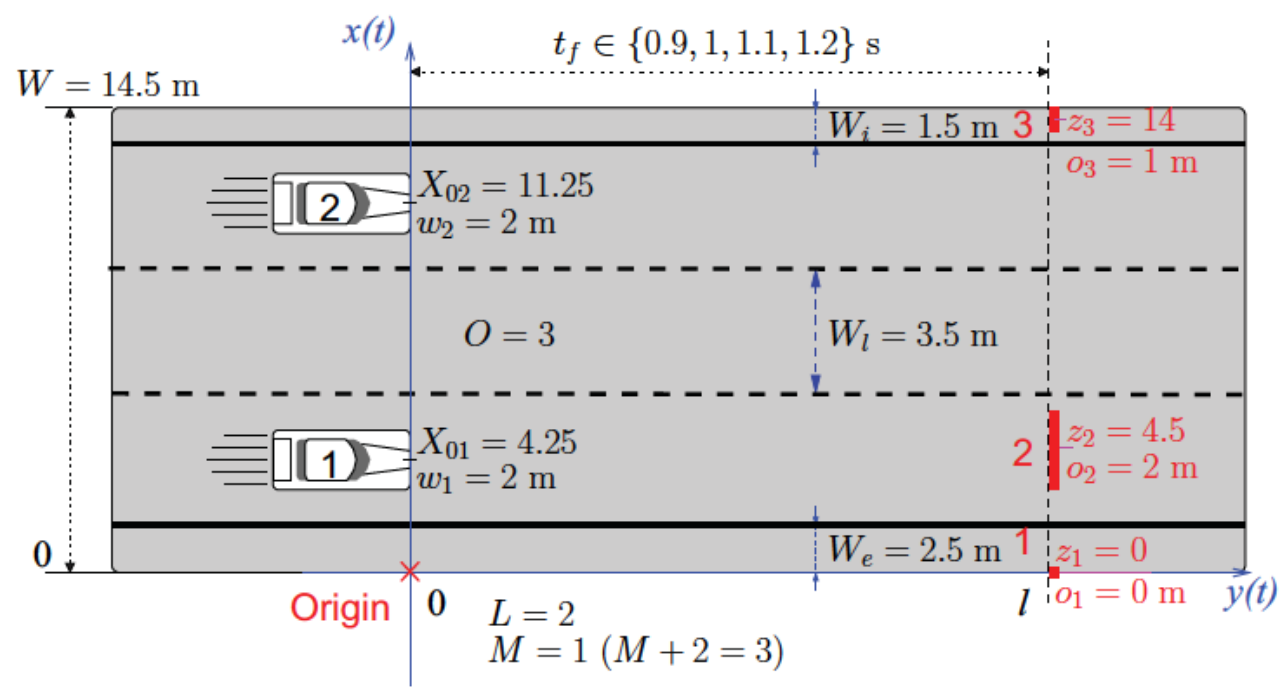

Figure 6.9: Particular scenario used for evaluation

We focus on the analysis of the scenario shown in Fig. 6.9, where $L=2$ vehicles circulate at $v_{I} \mathrm{~km} / \mathrm{h}$. At $t=0$ both vehicles discover $M+2=3$ obstacles $(M=1$ central object and the 2 default crash barriers) on the road $l$ meters (or $t_{f}=\frac{l}{v_{I}}$ seconds) ahead. In particular, the value of the maximum allowable lateral acceleration we set up is taken from (104) $\left(c\left(v_{I}\right)=5.5432 \mathrm{~m} / \mathrm{s}^{2}\right)$, using the regression model of Table 1 in (104) for $\Gamma^{\prime}=9.42 \mathrm{~m} / \mathrm{s}^{2}$ and $\Delta C^{\prime}=3.56 \mathrm{~km}^{-1}$ (Fast Driving conditions). These values are based on an average estimation of the extreme steering capabilities extracted from multiple test-benches of a Renault Laguna on a Renault test track in France (see (104)). 


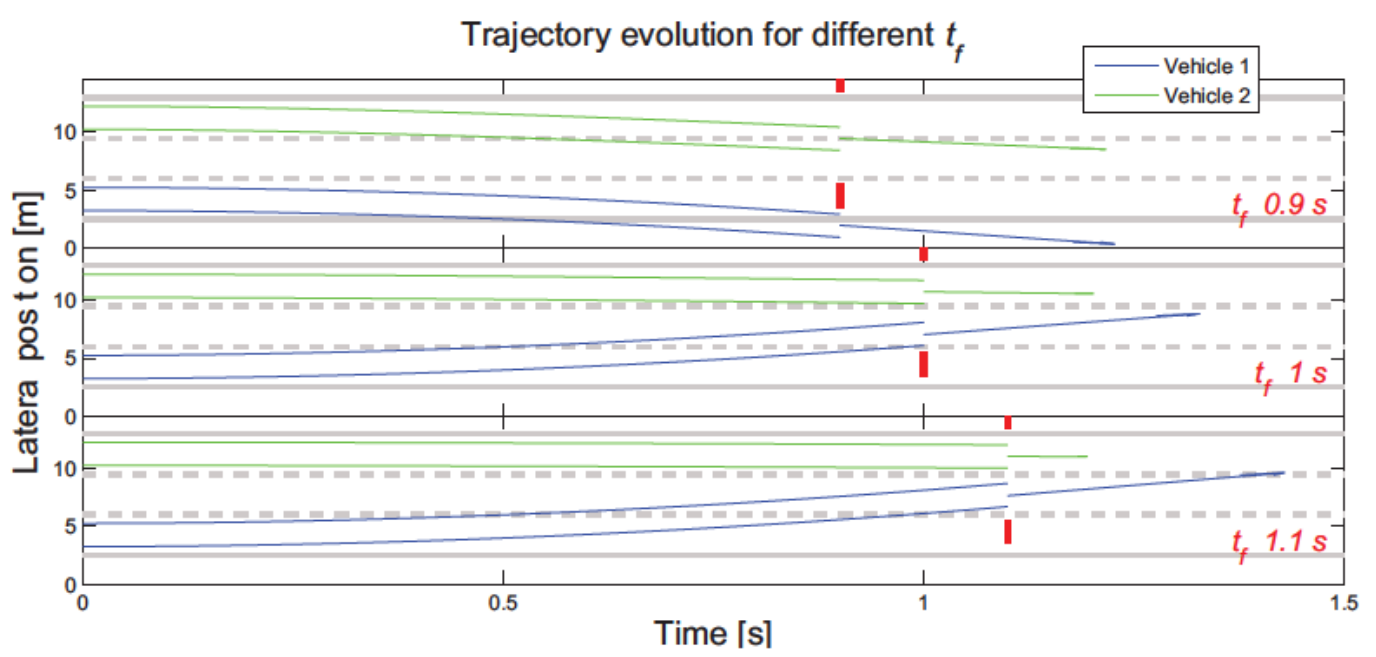

Figure 6.10: Evolution of trajectories for $M=1$ ( $M+2=3$ obstacles), $L=2$ vehicles and $\theta_{x}=1, \theta_{v}=0, \theta_{a}=0, t_{f} \in\{0.9,1,1.1\} \mathrm{s}$

On the road dimensions, we choose the corresponding dimensions found in (105), where there are 3 lanes, $3.5 \mathrm{~m}$ wide each, with external and internal edge measuring $2.5 \mathrm{~m}$ and $1.5 \mathrm{~m}$ respectively. We will divide the evaluation into three subsections, each one corresponding to the influence of the $\theta=\left[\theta_{x}, \theta_{v}, \theta_{a}\right]$ factors (which are the weights of the subfunctionals $f_{m, x}, f_{v}$ and $f_{a}$, respectively). The general configuration of parameters for the three evaluation cases is shown in Table 6.1 (see also the Nomenclature at the end of this Chapter). The choice of the values for $\theta=\left[\theta_{x}, \theta_{v}, \theta_{a}\right]$ is arbitrary and aims at showing the flexibility that this procedure provides. Optimizing these parameters according to a previously chosen evasive strategy will be examined in a future work.

Next we will show the trajectories traced by both vehicles in each case, as well as the mobility profiles of each one (speed and acceleration), along with a detailed analysis of the results at hand. Trajectories will show the sweeping area traced by each vehicle on the projected axis and its evolution on the 2 D-plane ${ }^{1}$ as shown in Fig. 6.9 by incorporating the longitudinal mobility. They will cover the time interval $t \in\left[0, t_{f}\right]$, i.e, since they start the maneuvers until they reach the longitudinal position of the obstacles (the Inertial Vector will also be represented for each single path). Acceleration profiles will only be traced for the instants $k \in[0, N-1]$ since the optimization requires to find the controls for the first $N$ steps $\left(a_{j}(N)\right.$ does not influence the trajectories). Obstacles

\footnotetext{
${ }^{1}$ The representation of trajectories will be made in two-column graphs so as to help for the interpretation of the curves.
} 


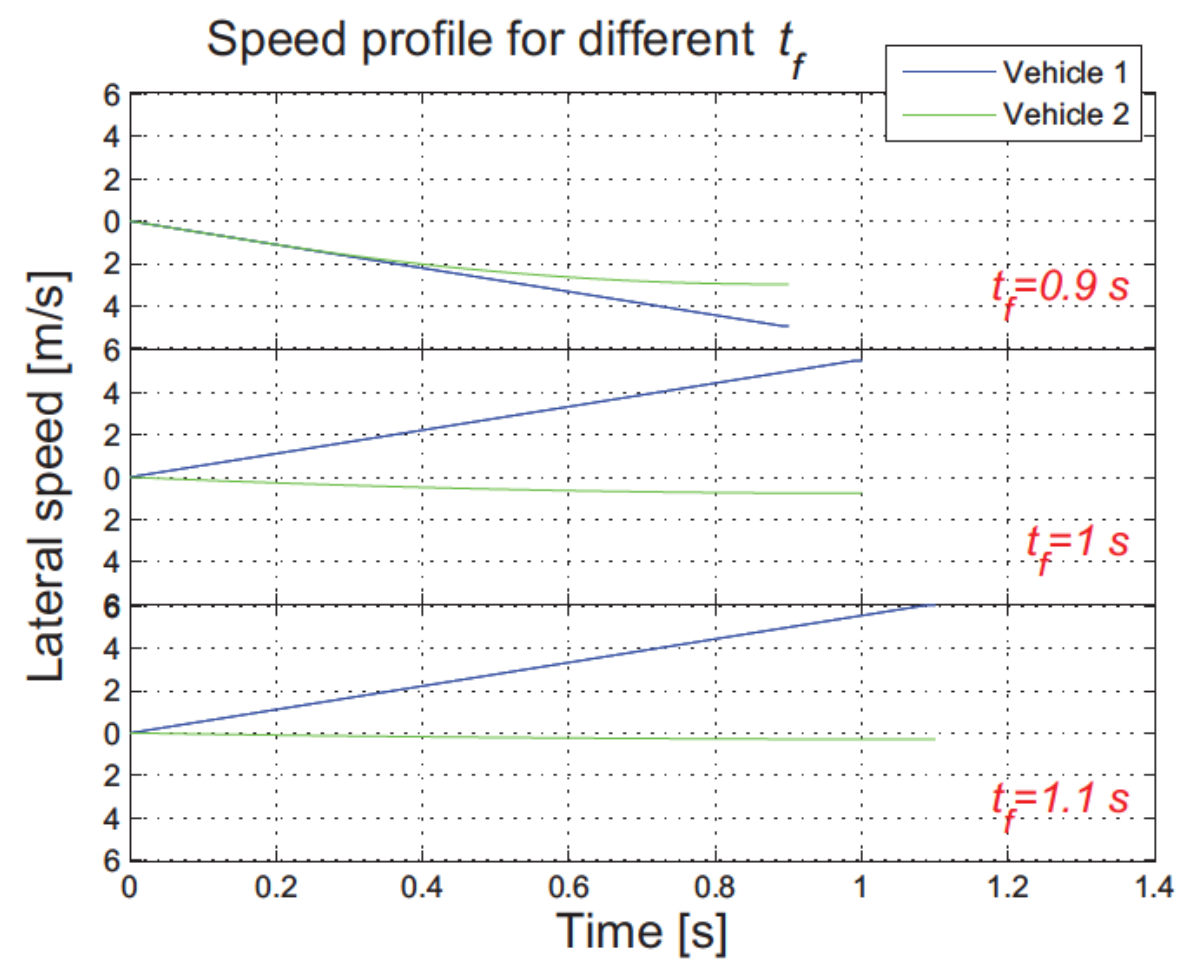

Figure 6.11: Speeds for $\mathrm{M}=1, \mathrm{~L}=2$ and $\theta=[1,0,0], t_{f} \in\{0.9,1,1.1\} \mathrm{s}$

will be printed as red lines and, lanes and external/internal edges will be delimited in graphs by corresponding gray (dashed) lines when applicable. The software Matlab (version 7.10.0, R2010a) is used to perform all the mathematical calculations.

\subsubsection{Influence of $\theta_{x}$}

Considering that the most important aspect to take into account when calculating the optimum trajectories is the maximization of the lateral distance between elements (vehicles and objects), we first evaluate the influence of $\theta_{x}$ for different values of $t_{f}$. We assume that $\theta_{v}=0$ and $\theta_{a}=0$, i.e. just implying that only lateral distance maximization $\left(\theta_{x}\right)$ is required. The rest of the parameters' configuration is shown in Table 6.1.

If we have a look at Fig. 6.10, we can see how trajectories are traced for $t_{f} \in$ $\{0.9,1,1.1\}$ s. It is obvious that the situation for Vehicle 2 is intuitively better than that of Vehicle 1: whereas Vehicle 2 does not have any obstacle directly ahead, Vehicle 1 has to unavoidably decide between turning to Gap 1 or heading for Gap 2 and sharing 


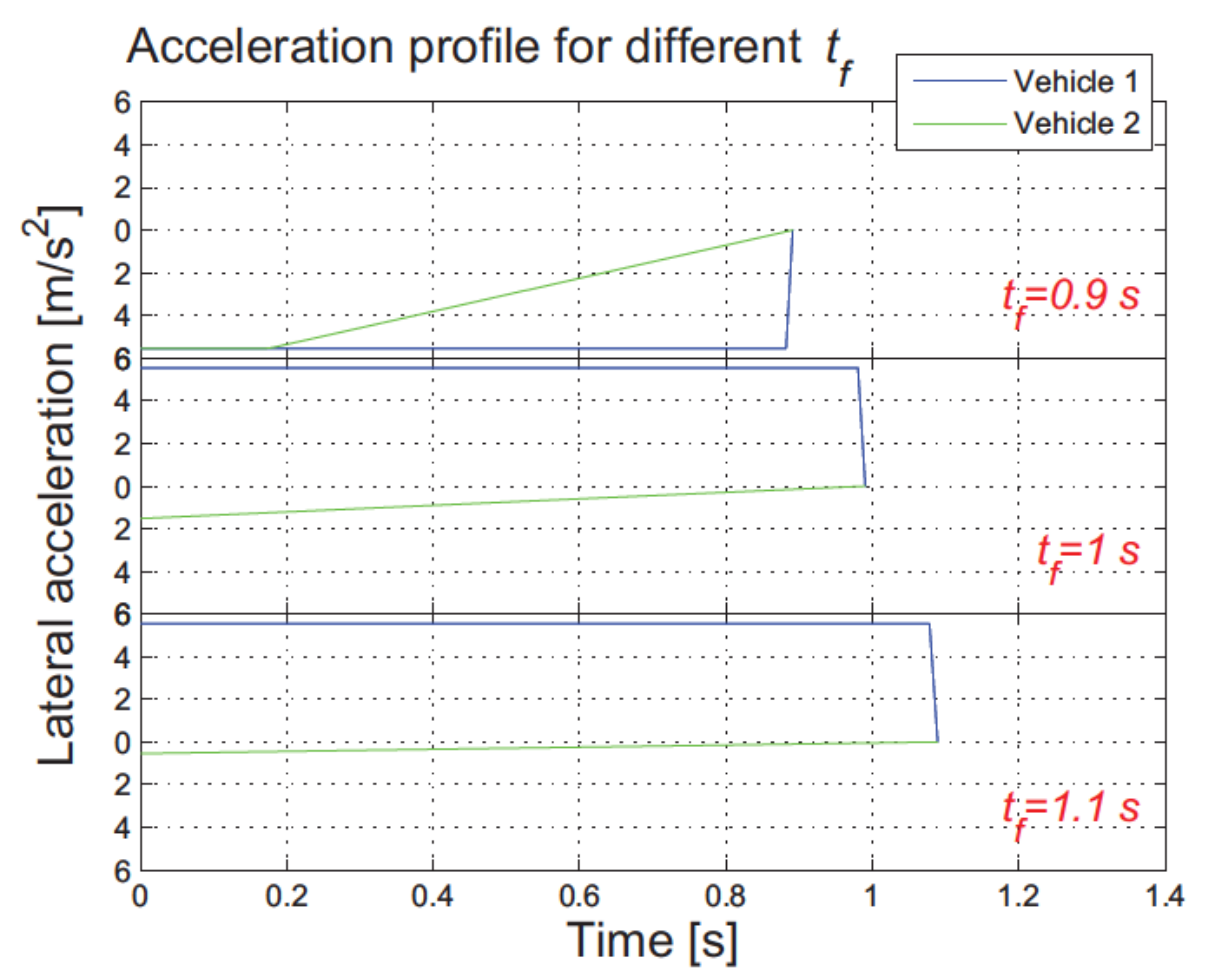

Figure 6.12: Accelerations for $\mathrm{M}=1, \mathrm{~L}=2$ and $\theta=[1,0,0], t_{f} \in\{0.9,1,1.1\} \mathrm{s}$

the space with Vehicle 2 in order to avoid the collision with Obstacle 2 (see Fig. 6.9). For the case $t_{f}=0.9 \mathrm{~s}$, Vehicle 1 only has $0.9 \mathrm{~s}$ to execute the maneuver successfully, so it decides to select Gap 1 (if Gap 2 was chosen, the short time to turn would imply a collision against Obstacle 2). In the other two cases, namely $t_{f}=1$ and $1.1 \mathrm{~s}$, vehicle 1 has already enough time to turn to Gap 2 without colliding with Obstacle 2, therefore sharing the lateral space with Vehicle 2, and maximizing the lateral distance.

In this particular case, the Inertial Vectors are represented with a modulus scaled to $1 / 3$ of its associated lateral speed (for visualization purposes). As we can see in Fig. 6.10 , the Inertial Vector of Vehicle 1 has a larger angle as well as a greater modulus than the Inertial Vector of Vehicle 2 showing that in all cases the maneuver of Vehicle 1 is harder than that of Vehicle 2. Although collisions with obstacles are avoided at the moment of reaching their position, having Inertial Vectors with large angles and modulus can be potentially risky due to the inertial motion that the vehicles will have after that, increasing the probability of lateral crashing between them and/or with the crash barriers. 


\section{COOPERATIVE COLLISION AVOIDANCE BY ACTIVE EVASIVE MANEUVERING}
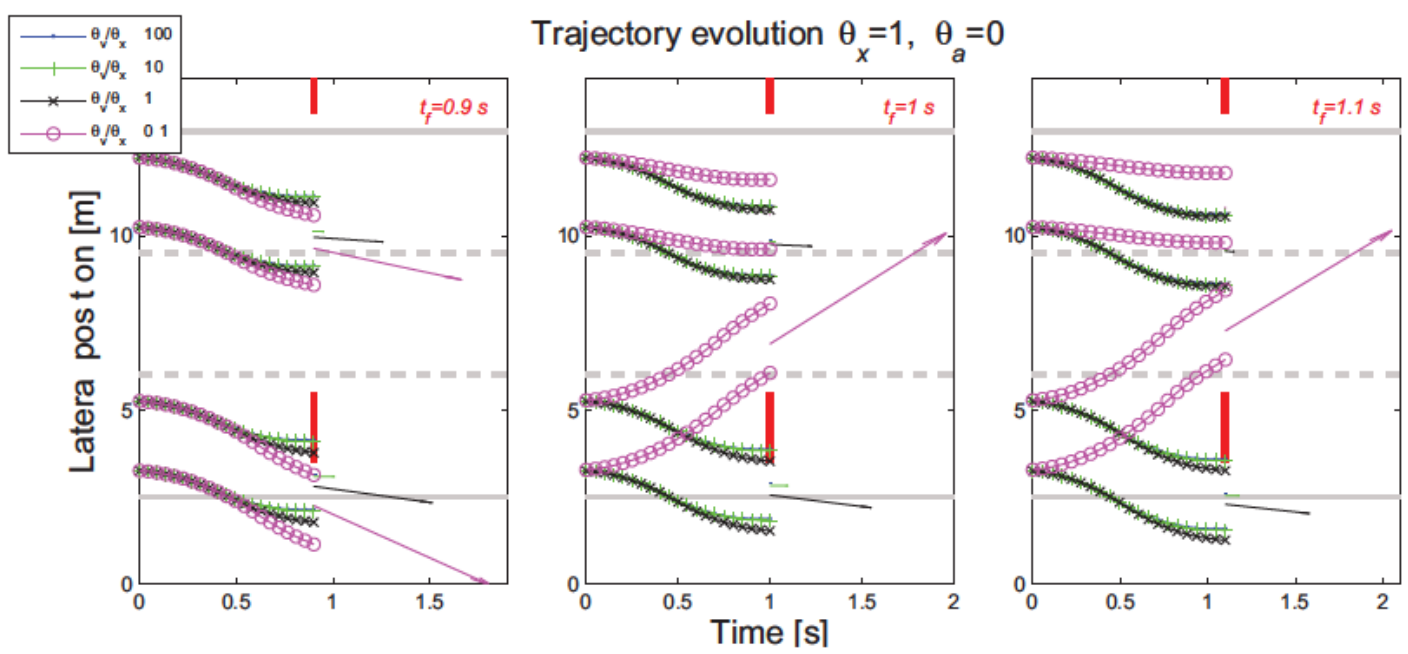

Figure 6.13: Evolution of trajectories for $M=1$ ( $M+2=3$ obstacles), $L=2$ vehicles and $\frac{\theta_{v}}{\theta_{x}}=\{100,10,1,0.1\}, \theta_{a}=0, t_{f} \in\{0.9,1,1.1\} \mathrm{s}$

The mobility profiles of both vehicles are illustrated in Figs. 6.11 and 6.12, showing that for $\theta=[1,0,0]$ the acceleration evolution needs to take the extreme values of the maximum acceleration set for the model $\left(5.5432 \mathrm{~m} / \mathrm{s}^{2}\right)$ to head for the optimum lateral position (Vehicle 1). If the trajectory does not require extreme accelerations, a smooth evolution of the acceleration profile is obtained (Vehicle 2). In general we see that the final lateral speeds of both vehicles are not null (except for the final velocity of Vehicle 2 when the trajectory slightly needs to be changed, particularly for the cases $t_{f}=1$ and $1.1 \mathrm{~s})$.

\subsubsection{Influence of $\theta_{v}$}

The main purpose of this subsection is to show the behavior of trajectories according to the specific configuration of $\theta_{v}$ respect to $\theta_{x}$, namely for the following cases: $\frac{\theta_{v}}{\theta_{x}}=\{100,10,1,0.1\}, \theta_{a}=0$. In Fig. 6.13 we show the evolution of trajectories for the aforementioned cases and for $t_{f} \in\{0.9,1,1.1\}$. Our objective here is to evaluate primarily how Vehicle 1 behaves according to the specific configuration we set. Longitudinal dimensions in the graph are compressed in order to highlight the differences in the lateral motion (specifically for the trajectories). What we can observe, first for Vehicle 1 , is that the higher the ratio $\frac{\theta_{v}}{\theta_{x}}$, the smaller the final lateral inertia at the end of the path (see the corresponding Inertial Vectors). However, the risk of crashing with the obstacles is also higher (it is more difficult to reach the optimum lateral position): 


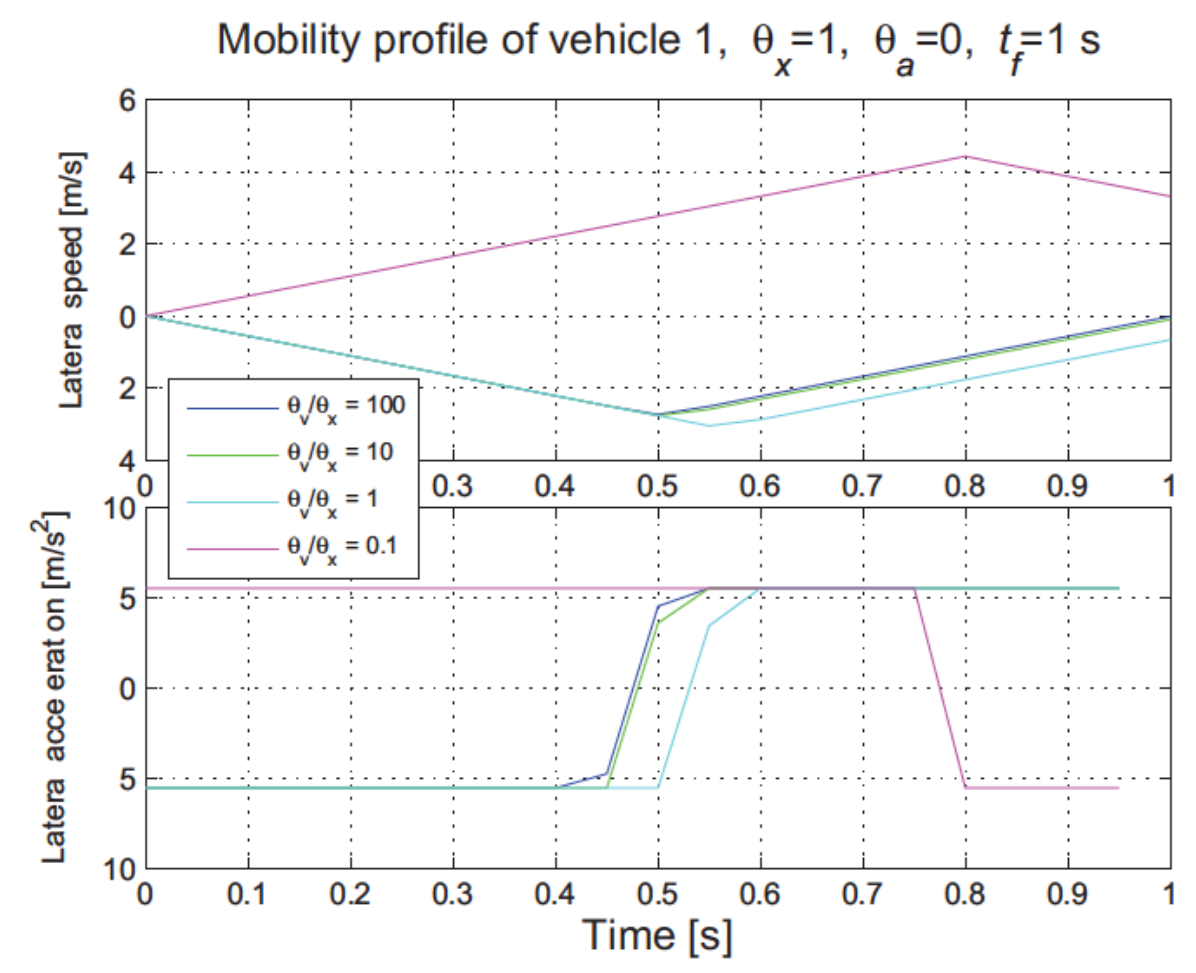

Figure 6.14: Mobility profile of vehicle $1, \frac{\theta_{v}}{\theta_{x}}=\{100,10,1,0.1\}, \theta_{a}=0, t_{f}=1 \mathrm{~s}$

actually collisions happen as can be seen in Fig. 6.13 for all the ratios except $\frac{\theta_{v}}{\theta_{x}}=0.1$, for Vehicle 1. Thus, it is clear that for lower values of $t_{f}$, Vehicle 1 urgently needs to use a lateral acceleration as high as possible in order to skip the collision. Speed and acceleration profiles can be seen in Fig. 6.14, which clearly shows that the higher the $\frac{\theta_{v}}{\theta_{x}}$ ratio, the better the compensation of accelerations in order to reach null lateral inertia at the end of the path (in these particular cases, these acceleration-compensated trajectories show similarities with the VDTs obtained in the work of (90)). The lower this ratio, the more similar is the behavior to the cases of the last subsection (less minimization of lateral speed at the end of the trajectory, at $t_{f}$ ).

Vehicle 2, on the other hand, always heads for the optimum lateral positions with lower values of the Inertial Vector, since its immediate gap is not blocked by any obstacle located directly ahead (Fig. 6.13). The higher the value of $t_{f}$, the smaller will be the modulus and angle of the Inertial Vector, i.e. the less inertial influence at the end of the maneuver. Actually, we can see in the mobility profiles that accelerations take the two extreme values during the time interval to execute the maneuver (Fig. 6.15), thus 


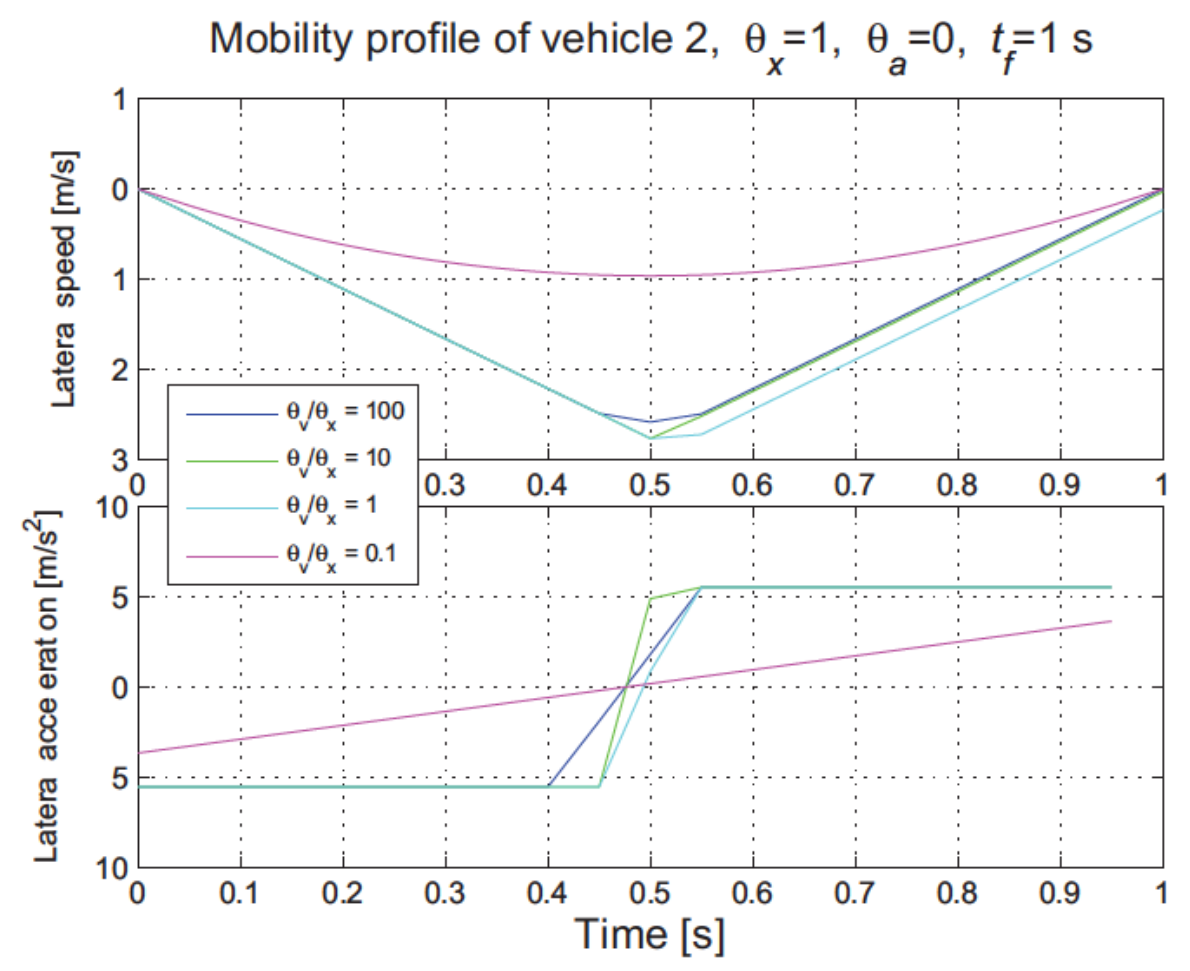

Figure 6.15: Mobility profile of vehicle $2, \frac{\theta_{v}}{\theta_{x}}=\{100,10,1,0.1\}, \theta_{a}=0, t_{f}=1 \mathrm{~s}$

reaching the peak value of the speed at the middle of the time period (see Fig. 6.14).

In summary, including the lateral speed in the functional allows to effectively control the lateral inertia when there is enough time before reaching the position of the obstacles. However, controlling the lateral distance to the obstacles should be given maximum priority (for obvious reasons), which can be flexibly set up with the use of different weights.

\subsubsection{Influence of $\theta_{a}$}

It is usual in the literature to find works $(90,95)$ which study how to develop laws of motion intended to minimize the impact of sudden accelerations on the passengers of vehicles. In this subsection we study how the term corresponding to the $\theta_{a}$ factor can influence the maneuvers under the critical circumstances of the scenario depicted in Fig. 6.9. As well as in the last subsection, we highlight the lateral changes in the mobility by compacting the longitudinal dimension of the road. Taking as reference the value $\theta_{x}=1$, we will represent trajectories for all the combinations of the two sets 


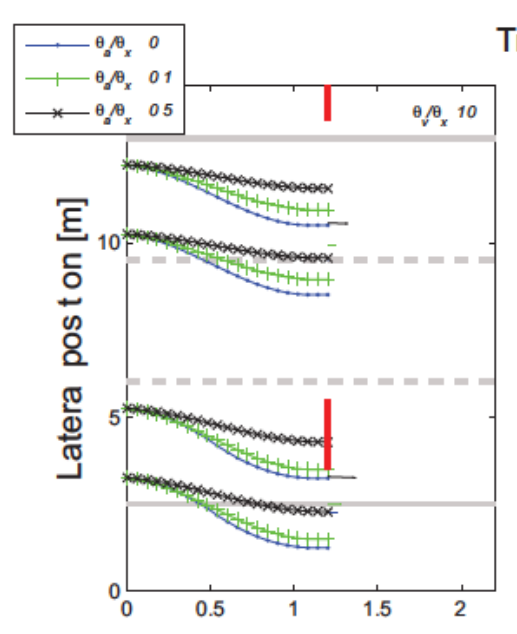

Trajectory evolution, $\theta_{x}=1, t_{f}=1.2 \mathrm{~s}$
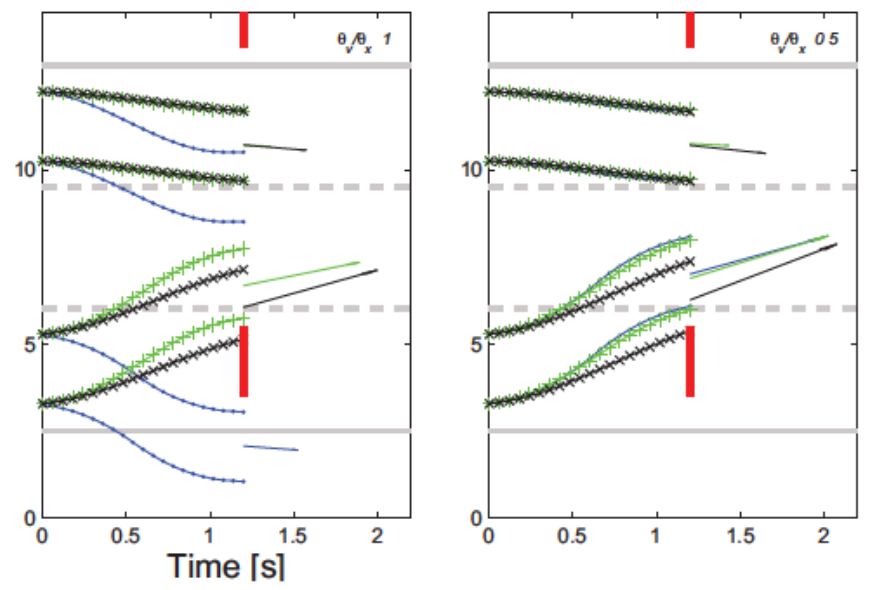

Figure 6.16: Evolution of trajectories for $M=1$ ( $M+2=3$ obstacles), $L=2$ vehicles and $\frac{\theta_{v}}{\theta_{x}}=\{10,1,0.5\}, \frac{\theta_{a}}{\theta_{x}}=\{0,0.1,0.5\}, \theta_{x}=1, t_{f}=1.2 \mathrm{~s}$

$\frac{\theta_{v}}{\theta_{x}}=\{10,1,0.5\}$ and $\frac{\theta_{a}}{\theta_{x}}=\{0,0.1,0.5\}$, for a $t_{f}=1.2 \mathrm{~s}$.

Results in Fig. 6.16 show that with increasing values of the ratio $\frac{\theta_{a}}{\theta_{x}}$, the less brusque every maneuver is. Of course, the dependence of the inertial state of the vehicle at the end of the path due to $\frac{\theta_{v}}{\theta_{x}}$ requires this fraction to be higher so as to reduce the modulus of the Inertial Vector of each trajectory. We can even observe that for certain cases (in particular, look at the case $\frac{\theta_{v}}{\theta_{x}}=1, \frac{\theta_{a}}{\theta_{x}}=0.1$, Fig. 6.16) the trajectory of Vehicle 1 changes the Gap (respect to the case $\frac{\theta_{a}}{\theta_{x}}=0$ ) because the smoothened trajectory does not have any other option but choosing this possibility in order to both minimize distance variance and minimize the sum of the square of accelerations along the path for the given $\theta_{a}$. The problem is that as this ratio becomes higher, the possibilities of crashing are also higher (for example, there is a collision for Vehicle 1 in case $\frac{\theta_{v}}{\theta_{x}}=1$, $\frac{\theta_{a}}{\theta_{x}}=0.5$, Fig. 6.16). Figs. 6.17 and 6.18 show the evolution of accelerations and speeds for Vehicle 1. It can be seen the correspondence with the trajectories of Fig. 6.16, where lateral speeds are smoother with higher values of the fraction $\frac{\theta_{a}}{\theta_{x}}$, as expected.

In summary, again the proposed acceleration component of the functional meets its goal of smoothing the accelerations suffered by the passengers, but it also has to be carefully weighted with respect to the other criteria in order to ensure safety. A more detailed study on the values and ratios of the three weights is left as future work as previously mentioned. 


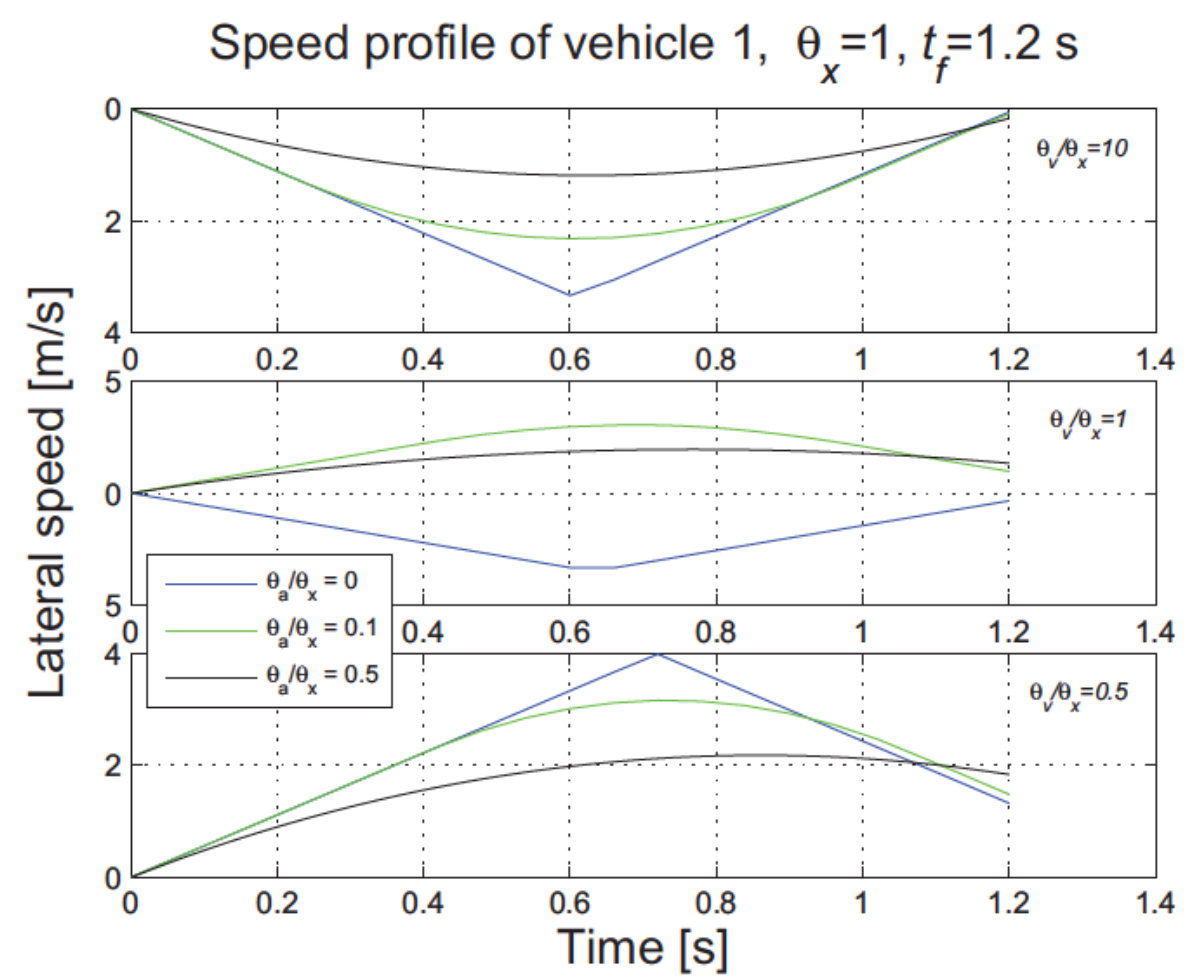

Figure 6.17: Speed profiles of vehicle 1 , for $\mathrm{M}=1, \mathrm{~L}=2$ and $\frac{\theta_{v}}{\theta_{x}}=\{10,1,0.5\}, \frac{\theta_{a}}{\theta_{x}}=$ $\{0,0.1,0.5\}, \theta_{x}=1, t_{f}=1.2 \mathrm{~s}$

\subsection{Conclusions and future work}

In this Chapter we have presented a procedure for the computation of trajectories under critical circumstances like those of Fig. 6.1, in the context of CCA applications for evasive maneuvering. We pose it as an optimal control problem and simplify it by considering only lateral motion, assuming longitudinal speed constant, what we call $x$-axis projection, thus having to solve a one-dimensional trajectory tracing problem. This simplification is brought up as a worst case scenario where vehicles have to share the lateral space to overcome the obstacles appearing ahead. For small values of $t_{f}$ this is reasonable, but it can be expected that the use of brakes in longitudinal motion may increase safety in such dangerous events. We intend to extend the current problem in the future by incorporating longitudinal acceleration and establishing specific patterns of motion for the optimization of the trajectories, while simultaneously keeping an affordable computational cost for the calculation process.

We introduced a performance measure to optimize the routes of vehicles $\left(J_{L, M}\right)$ 


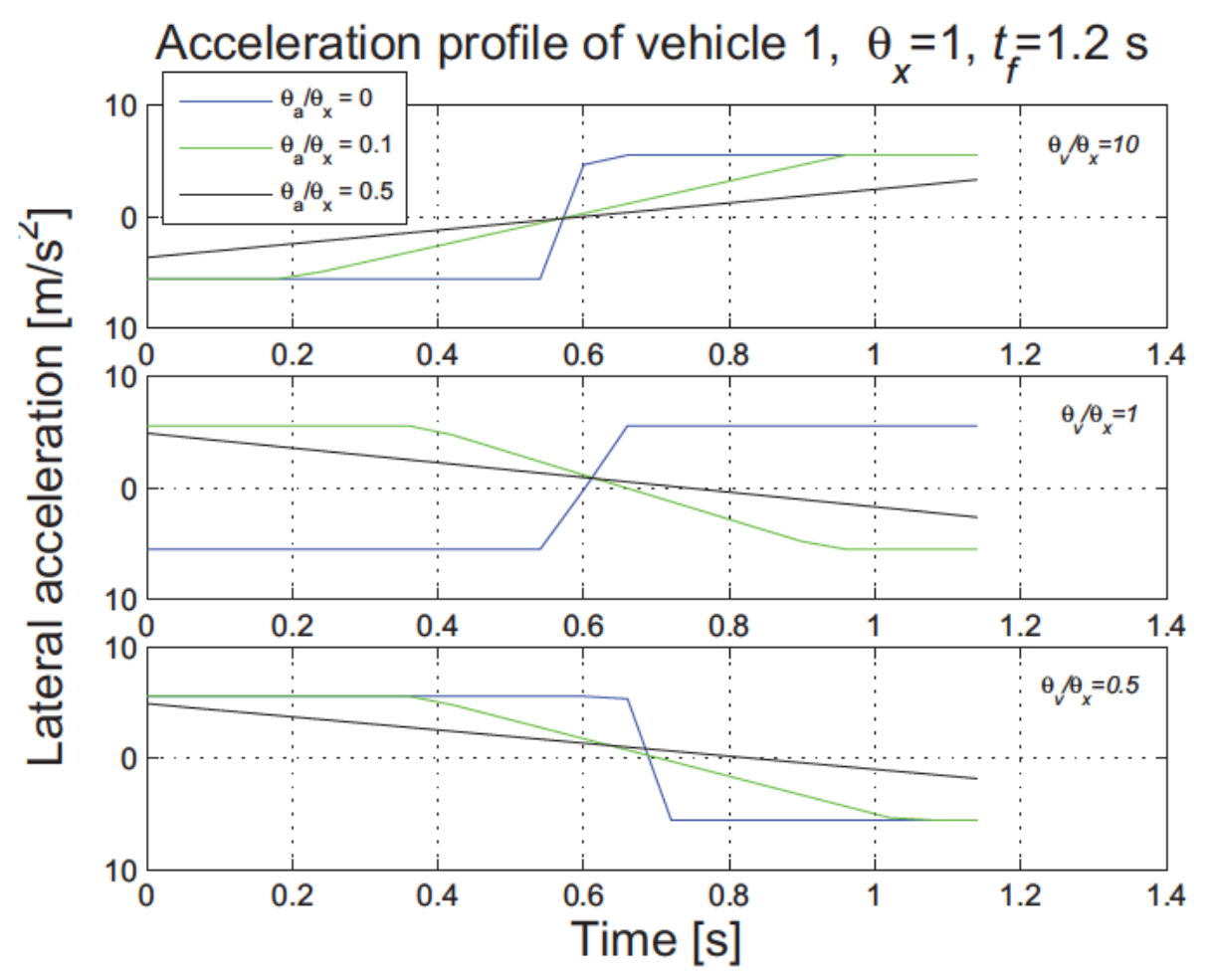

Figure 6.18: Acceleration profiles of vehicle 1, for $M=1, L=2$ and $\frac{\theta_{v}}{\theta_{x}}=\{10,1,0.5\}$, $\frac{\theta_{a}}{\theta_{x}}=\{0,0.1,0.5\}, \theta_{x}=1, t_{f}=1.2 \mathrm{~s}$

according to three different weighted criteria: we can choose to combine the minimization of the lateral distance variance, the minimization of the final lateral speed and the maximum reduction of the total acceleration along the path, by setting the weights $\left[\theta_{x}, \theta_{v}, \theta_{a}\right]$. Results in Section 6.4 show the great flexibility that autonomous vehicles have to trace evasive maneuvers in critical situations according to these three optimization requirements. However, it must be done carefully as some combinations might result in dangerous trajectories. Therefore, a future work is to analyze and optimize the combination of the three parameters $\left[\theta_{x}, \theta_{v}, \theta_{a}\right]$ for different scenarios of application.

Although based on a gradient-descent approach, the Modified Gradient Projection (MGP) method is appropriate to perform the local optimization because the number of subproblems to optimize should not be too large in a real situation, each one converges quickly and does not require too much computing power. However, there is still a number of procedures of the algorithm that can be studied and fine-tuned in order 


\section{COOPERATIVE COLLISION AVOIDANCE BY ACTIVE EVASIVE MANEUVERING}

to optimize the execution time, as for instance, the discretization factor $N$. There is one drawback, however: due to the non-convexity of the problem, the processing time to reach the closest local solution might be too large if the descent rate is too low or because of a lack of convergence. In future works the way the processing step is updated will be profoundly studied.

Finally, we have shown here a centralized version of the algorithm, that is, we assume that perfect position information is available for a central node able to compute the optimal trajectories and deliver them to the rest of vehicles. Developing a distributed version is a future work as well. There are different options for implementing a distributed version to be studied. A straightforward approach could be to select a car to act as coordinator and central node, or since our optimization strategy minimizes separate subfunctionals (with MGP), they can be split among the participating nodes. In any case, additional information exchange procedures have to be incorporated and there are issues to solve. For instance, the execution of cooperative maneuvers needs very low time delays from the first instant when the obstacle is monitored until the maneuvers begin. Indeed, this work was originally conceived to include a communication protocol for a distributed CCA application in situations like the one already described. Hence, other option to advance further in the research associated to this work is to evaluate in detail the maximum admissible time delays for such a CCA application to work properly under most situations. This is a critical issue since the calculation of trajectories is only useful if the associated maneuvers can be started within very short time delays, for obvious reasons.

\section{Nomenclature}

$L \quad$ Number of vehicles.

$M \quad$ Number of intermediate obstacles $(M \geq 0)$.

$N \quad$ Number of steps, discretization factor.

O Number of lanes.

$W_{l} \quad$ Lane width $[\mathrm{m}]$.

$W_{i} \quad$ Width of the internal edge of the road $[\mathrm{m}]$.

$W_{e} \quad$ Width of the external edge of the road [m].

$W \quad$ Total road width: $O \times W_{l}+W_{i}+W_{e}[\mathrm{~m}]$. 
$j \quad$ Subindex to refer to vehicle $j \in\{1, \ldots, L\}$.

$\beta \quad$ Subindex to refer to obstacle $(\beta \in\{1, \ldots, M+2\})$, or gap $(\beta \in\{1, \ldots, M+1\})$.

$k \quad$ Step instant for each trajectory, $k \in\{1, \ldots, N\}$.

$t_{f} \quad$ Maneuverability time interval [s].

$v_{I} \quad$ Longitudinal speed $[\mathrm{m} / \mathrm{s}]$.

$l \quad$ Distance to obstacles $[\mathrm{m}]$.

$\Delta t \quad$ Discretized time lapse $\Delta t=\frac{t_{f}}{N}$.

$x_{j}(t) \quad$ Lateral position of vehicle $j[\mathrm{~m}]$.

$w_{j} \quad$ Width of vehicle $j[\mathrm{~m}]$.

$v_{j}(t) \quad$ Lateral speed of vehicle $j[\mathrm{~m} / \mathrm{s}]$.

$a_{j}(t) \quad$ Lateral acceleration of vehicle $j\left[\mathrm{~m} / \mathrm{s}^{2}\right]$.

$z_{\beta} \quad$ Position of obstacle $\beta[\mathrm{m}]$.

$o_{\beta} \quad$ Width of obstacle $\beta[\mathrm{m}]$.

$X_{0 j} \quad$ Initial lateral position of vehicle $j[\mathrm{~m}]$.

$V_{0 j} \quad$ Initial lateral speed of vehicle $j[\mathrm{~m} / \mathrm{s}]$.

$A_{0 j} \quad$ Initial lateral acceleration of vehicle $j\left[\mathrm{~m} / \mathrm{s}^{2}\right]$.

$v_{I} \quad$ Longitudinal speed $[\mathrm{m} / \mathrm{s}]$.

$c\left(v_{I}\right) \quad$ Function to calculate the maximum lateral acceleration (from the longitudinal speed) $\left[\mathrm{m} / \mathrm{s}^{2}\right]$.

$J_{L, M} \quad$ General performance measure.

$S(t) \quad$ Set of $x$-axis projections of the positions of the $L$ vehicles.

$V \quad$ Set of $x$-axis projections of the positions of the $M+2$ obstacles.

$\gamma_{i}(t) \quad i$-th element of the set $S(t) \cup V$.

$\tilde{\sigma}_{\text {dist }}^{2} \quad$ Distance variance function.

$\sigma_{\text {dist }}^{2} \quad$ Function to calculate the square-sum of lateral distances (adapted distance variance).

$\theta_{x} \quad$ Weighting factor of $f_{x}, f_{m, x}$.

$\theta_{v} \quad$ Weighting factor of $f_{v}$.

$\theta_{a} \quad$ Weighting factor of $f_{a}$.

$R_{L, M+1} \quad$ Number of weak compositions ${ }^{1}$ of $L$ vehicles into $M+1$ gaps.

$m \quad$ Index to represent the $m$-th solution of the weak $(M+1)$-composition of $L$.

\footnotetext{
${ }^{1}$ In the text we will refer to this as composition or combination indistinctively.
} 


\section{COOPERATIVE COLLISION AVOIDANCE BY ACTIVE EVASIVE MANEUVERING}

\footnotetext{
$J_{L, M}^{(q)} \quad$ Subfunctional for the combination $q$.

$\overline{J_{L, M}} \quad$ Minimum value of the performance measure in the reformulation proposal.

$\sigma_{m, \text { dist }}^{2} \quad$ Square-sum of lateral distances for combination $m$ (adapted distance variance).

$G_{m, \beta}^{(L, M)} \quad$ Number of vehicles in gap $\beta$ for the composition $m$ (distribution of $L$ vehicles into $M+1$ gaps).

$\Xi_{m, l}^{(L, M)} \quad$ Number of accumulated vehicles for the first $l$ gaps in combination $m$ (for the distribution of $L$ vehicles into $M+1$ gaps).

$\phi_{m, \beta}(t) \quad$ Square-sum of lateral distances for vehicles between obstacles in gap $\beta$ of combination $m$.
} 


\section{7}

\section{General Conclusions}

\subsection{Main summary}

In this Work we have dealt mainly with the investigation of active policies for Collision Avoidance in vehicular scenarios at risk of accident. We decided to structure this Thesis Document into two differentiated parts, namely the design of applications for i) Cooperative chain Collision Avoidance and ii) Cooperative Collision Avoidance by active evasive maneuvering. During the nineties and the first decade of the 21 st century, the Automobile Industry accelerated the pace in innovation by clearly moving towards a rapidly progressive automation of the driving task for an enhanced driving experience, and a valuable increase in terms of safety. Manufacturers like Opel (55), Audi (106) or Mercedes-Benz (107), have done a great effort by means of experimentation to automatize driving by using Artificial Intelligence in vehicles equipped with advanced sensors and cameras. The main goal of this research is to make vehicles capable enough of operating on normal roads and complete routes with safety and efficacy. On the other hand, by the time the predoctoral stage started, the standard for communications in vehicular environments, WAVE 1609/802.11p, was being finalized (48), with its final draft appearing two years ago, in November 2010.

The obvious tendencies in investigation on next-generation vehicles comprised robotic driverless vehicles on the one hand, and vehicular connectivity by IEEE 802.11p, on the other (16). Besides, at the beginning of the Thesis, some companies not directly related to the Vehicular Industry started to take part in this game, carrying out extensive research on autonomous vehicular mobility as seen in (9). More importantly, even currently a more in-detail interest is being given to the analysis of situations (for 


\section{GENERAL CONCLUSIONS}

robotic assisted vehicles) where vehicles have to react to unpredictable situations like obstacle collision avoidance, pedestrian run-over avoidance, etc (12). Therefore, it is quite reasonable to use communications in self-guided cars as an advanced option to, not only restrict ITS capabilities to singular AI support in isolated cars, but also to provide more means of information which can serve to anticipate risky circumstances more efficiently.

As a result, the main decision of our group was to analyze a very common cause of multiple accidents like was that of chain collision avoidance in platoons of vehicles, and evaluate how the integration of communication protocols for an improvement in the anticipation of events could help for safety. We first analyzed the problem by means of simulation using a specific platform, NCTUns 6.0 (see Appendix A), which could give us a first impression about the benefits of using such technologies in the cited scenarios. With communications operating in platoons, accidents could be significantly reduced, as could be seen in the results of Section 3.3 in Chapter 3. We deduced that under some certain circumstances one-hop communications could be enough to deliver information timely to vehicles subject to risk of colliding, except for cars standing in the middle of the platoon, normally at high intervehicular distances and long chains of vehicles, for which some means of relaying could be not only reasonable, but necessary. Furthermore, we studied the influence of different penetration degrees of the technology in the market, by assessing how it could affect the number of chain collisions. We saw that the performance provided by CcCA applications could be severely reduced if the technological transition stage is not conveniently analyzed, due mainly to the complex situation caused by a heterogeneous mixture of vehicles with and without CcCA support. For this reason, in this case, AI in autonomous vehicles should be effectively prepared to account for these undesired phenomena. To validate our results we also developed a stochastic model which served us to analyze from a mathematical perspective the influence on the number of accidents of different design parameters like the initial speed $V_{i}$, the notification delay $\delta_{i}$, and the braking deceleration $a_{i}$ of the vehicles. Different cases were presented with a stochastic characterization of the corresponding scenarios, with design parameters behaving according to realistic probabilistic distributions chosen for this case. The abstraction of the notification delay $\delta_{i}$ proved to be really useful, since it gave us the opportunity to characterize different communication schemes, which is 
actually ideal to evaluate different communication schemes that could be used in these domains.

In Chapter 4 we tested the performance of three algorithmic solutions for packet relaying in the same scenarios evaluated in Chapter 3. We could understand better the behavior of CcCA applications and the improvement in performance when using multihop communications in those cases in which just one-hop schemes do not suffice. Using relaying would increase channel's occupation by filling it with possibly unnecessary data, but the success ratio in packet reception would also be higher. However, it might imply a higher latency in the end-to-end delay, a factor that can be really counterproductive in this sort of applications, thus justifying the use of one-hop communication schemes when relaying can be redundant. This, however, must be thoroughly studied in real manufacturing, since the choice between both schemes must be adaptive according to the specific scenario where vehicles operate. Therefore, an intelligent device that can analyze the channel in real-time and decide simultaneously which strategy is the best one, is still an open issue that will have to be evaluated in detail by manufacturers.

Chapter 5 introduced the IDM (Intelligent Driver Model) as a means to give a higher realism to simulations in our CcCA application as regards vehicular mobility. By using this strategy we provided vehicular entities with an advanced mobility model that implemented more degrees of freedom for the governing of the kinetic parameters of vehicles, i.e. a vehicle would react not only to the mobility of the front vehicle, but also according to multiple cars ahead. We also presented an additional algorithm for successfully brake management in scenarios of chain collision avoidance by introducing the LBA (Linear Braking Algorithm), which would integrate both safety and comfort in the task of driving. By using the acceleration variance, we made use of a reasonable performance metric that can usefully characterize the process of braking specially in terms of driving comfort. We deduced from this study that car-following models can manage mobility well under general stable circulation, but their performance can be severely degraded under situations like collision avoidance, where vehicles need to react in a non-linear manner to preserve safety (and secondly, comfort) of passengers and the car's body.

In the second part of the Thesis, we decided to focus our research on Cooperative Collision Avoidance (CCA) by evasive maneuvering. In this case, we assumed that vehicles would have in certain situations enough lateral space in scenarios where just 


\section{GENERAL CONCLUSIONS}

braking would not suffice to avoid the collisions. Thus, we developed in Chapter 6 an analytic method to obtain optimum maneuvers in situations where multiple vehicles have to avoid colliding with obstacles blocking their way at relatively short distances. Results showed that using such schemes can be highly beneficial in terms of passenger's safety, and at the same time regulate comfort according to the specific characteristics of the particular case, by controlling lateral acceleration and final lateral speed. A resolution algorithm was proposed to solve the problem by dividing the calculation process into a set of differentiable subproblems that can be treated by gradient-descent-based solvers. Communications aid in these matters, requiring very low latencies regarding end-to-end transmission delays, since vehicles need to execute maneuvers within very short intervals of time. In the Appendices we also included additional information on the networking platform used, NCTUns 6.0 (see Appendix A), specific mathematical operations for the stochastic model of Chapter 3 in Appendix B and the optimum control of evasive maneuvers of Chapter 6 (see Appendix C), and the calculation of optimum trajectories under the same premises of previously referred Chapter by introducing noise variability in the morphology of trajectories in Appendix D. Although out of the scope of the general objective of this Thesis, the latter Appendix showed briefly the need to implement active schemes to correct deviations in automatically calculated maneuvers caused by the presence of Gaussian additive noise.

\subsection{List of publications}

All the publications authored and co-authored by the Doctorand (journals, conference proceedings and book chapters) are listed next, with the most recent ones appearing first, and in descending order of date.

\subsubsection{Journal Papers}

- Tomas-Gabarron, J.B.; Egea-Lopez, E.; Garcia-Haro, J.; "Vehicular trajectory optimization for Cooperative Collision Avoidance at high speeds", IEEE Transactions on Intelligent Transportation Systems (under review, December 2012), (28). 
- Tomas-Gabarron, J.B.; Egea-Lopez, E.; Garcia-Haro, J.; "Optimization of vehicular trajectories under Gaussian noise disturbances", Future Internet, MDPI 2013, 5, pp.1-20 (27).

- Garcia-Costa, C.; Egea-Lopez, E.; Tomas-Gabarron, J.B.; Garcia-Haro, J.; Haas, Z.J.; "A Stochastic Model for Chain Collisions of Vehicles Equipped With Vehicular Communications", IEEE Transactions on Intelligent Transportation Systems, vol.13, no.2, pp.503-518, June 2012, (24).

- Tomas-Gabarron, J.B.; Egea-Lopez, E.; Garcia-Haro, J.; "Aplicaciones y futuro de las redes VANET (II): Servicios y aplicaciones", Mundo Electrónico, Grupo Tecnipublicaciones, Number 416, October 2009, pp.26-31. ISSN 03003787, (In Spanish), (21).

- Tomas-Gabarron, J.B.; Egea-Lopez, E.; Garcia-Haro, J.; "Aplicaciones y futuro de las redes VANET (I): 802.11p, estándar de comunicaciones inalámbricas para redes vehiculares", Mundo Electrónico, Grupo Tecnipublicaciones, Number 410, July 2009, pp.20-25. ISSN 0300-3787, (In Spanish), (20).

- Tomas-Gabarron, J.B.; Garcia-Haro, J.; "Protocolo MAC basado en identificadores para redes Ad-hoc vehiculares", Telecoforum 2008, ISSN 1698-2924, URI http://hdl.handle.net/10317/867, March 2009, (In Spanish), (108).

\subsubsection{Conference Proceedings}

- Tomas-Gabarron, J.B.; Egea-Lopez, E.; Garcia-Haro, J.; "Optimum maneuvering under time constraints for high speed vehicles", IEEE $2^{\text {nd }}$ International Workshop on Vehicular Communications and Networking (VECON 2012) in conjunction with the $8^{\text {th }}$ IEEE International Conference on Wireless and Mobile Computing, Networking and Communications (WiMob 2012), Barcelona, Spain, October 8, 2012, (26).

- Tomas-Gabarron, J.B.; Egea-Lopez, E.; Garcia-Haro, J.; "Evaluating communications and the Intelligent Driver Model in a Context of a Chain Collision Avoidance Application for VANETs", $3^{\text {rd }}$ International Conference on Road Safety and Simulation (RSS 2011), Indianapolis, USA, pp.13-15, September 15, 2011, (25). 
- Murcia, R.; Garcia-Costa, C.; Tomas-Gabarron, J.B.; Egea-Lopez, E.; Garcia-Haro, J.; "Parallelization of a mathematical model to evaluate a CCA application for VANETs", $1^{\text {st }}$ International Conference on Simulation and Modeling Methodologies, Technologies and Applications (SIMULTECH), The Netherlands (2011), (109).

- Tomas-Gabarron, J.B.; Egea-Lopez, E.; Garcia-Haro, J.; Murcia, R.; "Testing viability of relay policies for reactive CCA applications in VANETs", 2010 IEEE $6^{\text {th }}$ International Conference on Wireless and Mobile Computing, Networking and Communications (WiMob), pp.505-512, 11-13 Oct. 2010 doi: 10.1109/WIMOB.2010.5645020, (23).

- Tomas-Gabarron, J.B.; Egea-Lopez, E.; Garcia-Haro, J.; Murcia, R.; "Performance Evaluation of a CCA Application for VANETs Using IEEE 802.11p", 2010 IEEE International Conference on Communications (ICC), pp.1-5, 23-27 May 2010, (22).

- Tomas-Gabarron, J.B.; Egea-Lopez, E.; Garcia-Haro, J.; "Evaluación de mecanismos de priorización en 802.11p con VHDL", VII Jornadas de Ingenieria Telematica, Jitel 2009, ISBN: 978-84-96997-27-1, pp.190-196, Cartagena, 15-17, September 2009, doi: 10.1109/ICCW.2010.5503939,, (In Spanish), (46).

\subsubsection{Book Chapters}

- Garcia-Costa, C.; Tomas-Gabarron, J.B.; Egea-Lopez, E.; Garcia-Haro, Joan.; "Speeding Up the Evaluation of a Mathematical Model for VANETs Using OpenMP", Simulation and Modeling Methodologies, Technologies and Applications Advances in Intelligent Systems and Computing, 2013, Volume 197, Part 2, pp.23-37, DOI: 10.1007/978-3-642-34336-0_2, ISSN: 2194-5357, (110).

\subsection{Future worklines}

The future on automated interconnected vehicles is really promising. Although there have been many inventions and encouraging advances in this domain during the last years, it is now when this field of research attracts the most attention, and when a highest effort is required. To make vehicles intelligent enough to operate safely 
under every single circumstance is the most relevant goal of manufacturers nowadays. As could be described in the introductory part of this Book (Part I), complementing communications and self-guiding capabilities in vehicles is a hard task that still requires much work to be in the end commercially feasible. The Author firmly thinks that research on these matters is still in an initial stage, and has to be continued during the next decade.

As specific lines of continuity to this Work, the Author proposes to simulate the scheme for CCA in evasive maneuvering in some networking simulation platform like NCTUns 6.0, in order to either qualitatively as well as quantitatively evaluate the performance of different protocols to be developed. These referred protocols could be based on a centralized scheme where one vehicle, acting as a leader, could perform the calculations necessary to obtain the optimum routes for vehicles to follow. Other option might be to let these operations be distributed among the participating cars, allowing the parallel computation of trajectories for a better efficiency. These evaluations would require an in-depth study about their latencies in transmission delays for the different proposals, since CCA for evasive maneuvering requires to operate under highly tightening timing constraints.

Another option to study would be to integrate in the calculation of trajectories the natural variability to which maneuvers are subject in reality, as we did in Appendix D, but performing a thorough study of the matter and trying to imitate the most important premises of how recent developments in terms of probabilistic robotics are being used to manage mobility in the advanced autonomous prototypes of some manufacturers like Google (13). As an additional goal, the Author proposes to design an scheme to adapt the CCA (both for CcCA as for evasive maneuvering) to the normal functionality of an autonomous interconnected car for a seamless operation between normal road conditions, and abnormal critical circumstances.

Multiple different goals can be thought for research in collision avoidance, and all covering different multidisciplinary fields of vehicular technology (robotics, VANETs), but for sure, aiming at giving next car generations an added value to guarantee safety as has never been done before. 
7. GENERAL CONCLUSIONS 


\section{Appendix A}

\section{Over the NCTUns Network Simulator}

Network simulators are nowadays essential tools for the design, development and evaluation of communication protocols. The importance of simulation is due primarily to the fact that it allows developers to carry out experiments using a model-based approach that saves time and money. It also gives them much more flexibility than real experiments, since it can be used for example to evaluate network performance under different configurations with remarkable easiness. Simulation results are also easier to handle due to the great simplicity when directly collecting digital data and processing it.

Network simulators, however, have some limitations. A functional network simulator needs to simulate network devices (eg. routers and hosts) and applications that generate network traffic. It is also necessary to provide monitoring programs and set up the configuration parameters of each test. These facts mean that network simulators work according to a model of reality whose functionality might not correspond entirely with the real processes that it recreates. The associated drawbacks are summarized next:

- Network simulator results are not, for obvious reasons, as realistic as those obtained in real experiments under the same circumstances, since to limit the complexity and production costs, most network simulators implement simplified versions of reality (network protocols), that usually lead to results that do not necessarily match reality in all cases and circumstances. 


\section{A. OVER THE NCTUNS NETWORK SIMULATOR}

- Most network simulators do not have the property of extensibility because they are exempt from supporting the UNIX POSIX API ${ }^{1}$. As a result, existing network applications as well as those yet to be developed can not be executed properly to generate traffic for a given simulated network under different OS, nor can evaluate its performance under different network settings. On the contrary, they make use of the API provided by the own simulator and are compiled with it to build a unique, modular and quite complex program that only works in the same computer where compilation takes place.

To avoid these problems, the NCTUns/Estinet team, led by Prof. Shie-Yuan Wang (Department of Computer Science of National Chiao Tung University, Hsinchu in Taiwan) devised a simulation methodology called kernel reentering, which was already used in the first version of the simulator, called Harvard NS. Later, some improvements were made to the original methodology in order to finally implement the simulator and emulator capabilities that NCTUns currently supports $(112,113)$. By using the novel technique of kernel reentering, NCTUns provides a number of advantages over traditional simulators. Whatsoever, it also presents some disadvantages when programming, such as the steep learning curve for anyone who starts programming with NCTUns. Both advantages and drawbacks will be explained in detail in next sections.

\section{A.1 Kernel reentering methodology}

Using a tunnel network interface is the basic approach that summarizes the functionality offered by the reentering simulation methodology of NCTUns. A tunnel network interface available on most UNIX machines, is a pseudo network interface that does not possess a physical network interface assigned to it. However, the functionality of this interface is not different from the one offered by the Ethernet network interface, i.e. an application program to send and receive packets on this pseudo network interface would offer the same functionality as if it did through a physical interface.

Each tunnel interface has a special device file assigned to it, which any application running on NCTUns has access to, basically for writing information related to packets

\footnotetext{
${ }^{1}$ Family of standards which implements a common framework of system calls for extensive compatibility between different operating systems (see reference (111)).
} 

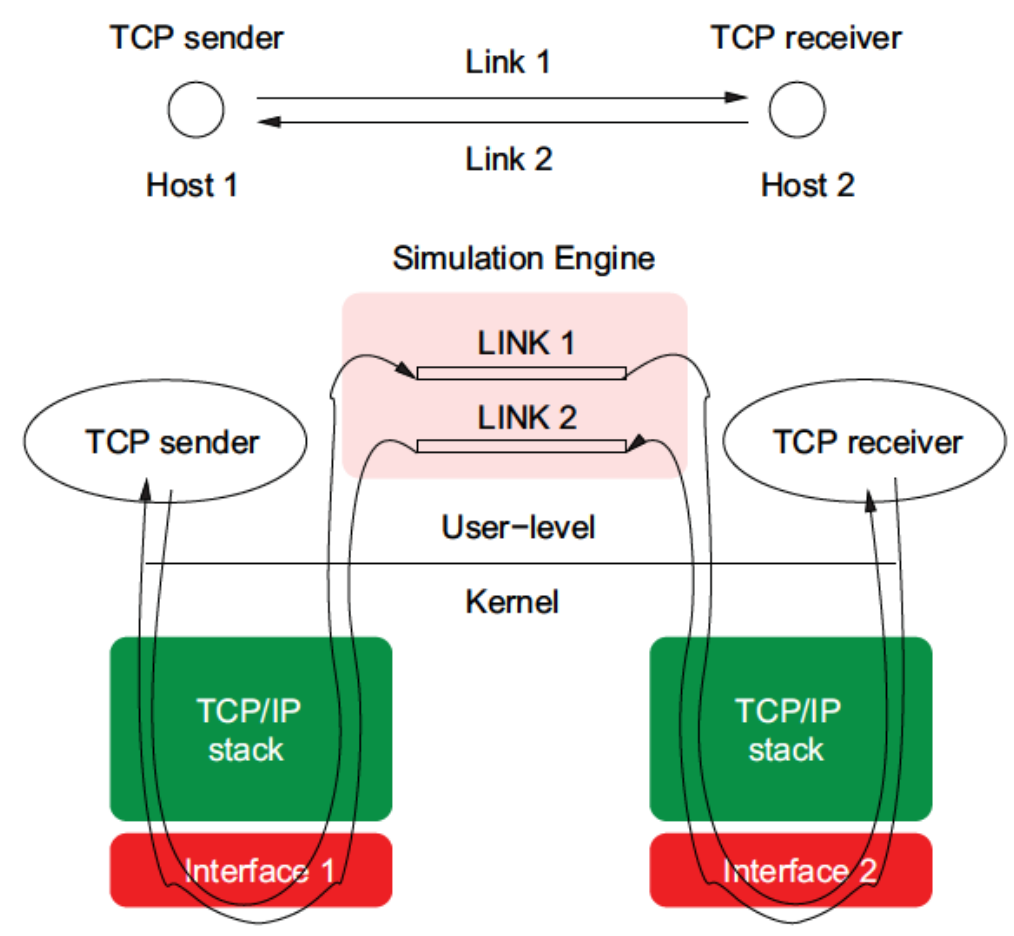

Figure A.1: Kernel reentering methodology of NCTUns

that are sent to other network entities for the purpose of simulation. From the perspective of the kernel module, the packet seems to come from an external communications network, and will come trough the TCP/IP protocol stack just like normal Ethernet packets would do (112). Moreover, if an application program reads a packet from the special device file of the tunnel interface, the first packet in the output queue of the kernel tunnel interface will be copied to the application program. This situation is interpreted by the kernel as if a packet had been sent to a real link without knowing if this transmission had taken place with real Ethernet packets (see Fig. A.1).

\section{A.2 Main characteristics of NCTUns}

In the following list we present the most important features that characterizes NCTUns as a network simulation platform, and the reasons we use to justify its choice as our simulation environment for performing our experiments along the first part of the Thesis.

- NCTUns uses the TCP/IP protocol stack to generate realistic simulation results. 


\section{A. OVER THE NCTUNS NETWORK SIMULATOR}

Thanks to the Kernel reentering methodology of NCTUns, this platform can use the UNIX protocol stack in its own network interface for the generation of very realistic results. In our case, since we will use WSMP as an alternative protocol network layer for the purpose of critical events notification, we will not take advantage of the Kernel reentering methodology provided by the platform (it still remains open as future work to make use of the TCP/IP stack to evaluate performance when generic background traffic is present).

- In NCTUns real UNIX applications can be used as traffic generators (teletraffic) for network performance evaluation. In our case, we will make the implementation of a specific application based on the timely transmission of chain collision alerts ( $\mathrm{CcCA})$.

- It is possible to use configuration tools and real network monitoring services such as ifconfig, netstat, tcpdump, traceroute, etc. available on all UNIX operating systems. In our case we will use a diagnostic tool designed exclusively by us, since our main concern is not to analyze the teletraffic utilizing the network, but until to which extent vehicle's safety can be improved.

- NCTUns supports the simulation of a wide variety of different types of network protocols and network devices. In our case we simulate a Vehicular Ad-hoc Network for emergency braking in CcCA (with the protocol stack architecture WAVE 1609/IEEE 802.11p).

- Due to the discrete event simulation approach of NCTUns, simulation runs execute very quickly (in the general case). Also, simulation results are repeatable, thus bringing us the possibility of evaluating network performance under particular scenarios that might need further analysis.

Unfortunately, NCTUns also has some drawbacks that may entail an increased difficulty when using this platform for the simulation of general computer networks:

- Although NCTUns is open source, it contains a proprietary GUI (Guided User Interface) that the end user can not modify. This somewhat reduces the flexibility of the programmer when declaring new nodes and/or developing new communication protocols. 
- NCTUns does not provide general simulation scripts for the automatic setup of network configuration files. In order to generate the results that we see along the Part I of this book, it was necessary to make a great effort to program a series of Bash/Python scripts that were used to set up network parameters in the VANET under consideration.

- The WSMP protocol we use for the transmission of information in the network layer of the WAVE architecture is not implemented in the Linux kernel. This implies that the advantage of directly using the TCP/IP stack from the network interface (basically, the reentering methodology) is lost due to the fact that WSMP is simulated as if it was a process of the application layer.

- The generation of a large number of sockets (for each virtual interface, each one for each vehicle) to perform simulations and the deficient memory release scheme of NCTUns when a simulation ends, specially after making a remarkable deal of system simulations, occasionally implies that the socket creation process takes a longer time than simulating the network under consideration.

- At the moment of using the NCTUns network simulator (two years ago), it suffered from a drawback that could negatively reduce the number of potential users: the lack of an API (Application Project Interface) documenting all the code generated for the networking platform, greatly increased the programmer's effort to understand the functionality offered by the simulator. Actually, the Author had to investigate the details of the code without having a sufficiently clear description of the architectural implementation of the NCTUns modules.

Fortunately, all these issues were solved during the last two years, by offering a commercial version of NCTUns, renamed Estinet, which provides the same functionality of NCTUns, but accounts for a very useful documentation of API that greatly reduces the effort of the network programmer. Whatsoever, it has become commercial, thus requiring to pay a license that might decrease the number of potential new users.

\section{A.3 CcCA Communications module for NCTUns}

Once we have presented the most important features of NCTUns, we present the main module implemented to support communications in applications for the transmission 


\section{A. OVER THE NCTUNS NETWORK SIMULATOR}

of chain collision alerts.

In the present case, we differentiate between two complementary modules:

- Mobility module: although NCTUns already includes the implementation of different types of mobility models, in our case we developed our own motion dynamics for vehicles driving in convoy under a significant risk of chain collision. We preferred to do this because already available mobility managers in NCTUns are adapted to govern vehicular motion on preestablished roads which must be configured beforehand in the setup files. In our case, we do not want to follow a certain path marked by a road, but analyze how vehicles can react when driving on a straight line and then they react to Cooperative chain Collision Avoidance (CcCA) notifications.

- Communications module: NCTUns brings us the chance to use the WSMP protocol (WAVE Short Message Protocol) as an alternative to the TCP/IP protocol stack for the transmission of safety information between vehicles. Because of the timing requirements set by this safety-related application, using this protocol is advisable in this case. In this context it is necessary to carry out some internal modifications of the original WSMP module ${ }^{1}$ in order to provide bidirectional contact between the communications module and the mobility module, as we will see next.

In our study, the implementation of two-way communication between the two modules is required, mainly for the following reasons:

- In a hypothetical situation where a vehicle collides with an obstacle along its path it is necessary to notify the communications module that it has the responsibility to send information to the following vehicles (in the form of WSMP packets) in order to inform them about this event.

- In the same situation, if a vehicle receives WSMP packets, a reaction to the information presented is foreseen. This requires informing both the communications module and the mobility module, so that the latter can respond to the received information by reducing the vehicle speed as appropriate.

\footnotetext{
${ }^{1}$ The one that came already installed in the NCTUns distribution.
} 
All vehicles will contain these two modules, coded by using the NCTUns APIs and the UNIX sockets libraries that hold the generic functionality of the AF_UNIX sockets, available for implementing communications between different processes on the same machine.

\section{A.3.1 Mobility module}

Due to the enormous complexity of developing mobility models for generic vehicular traffic, we focus on a particular implementation of a mobility model that is adapted to the specific situation of emergency braking for CcCA. In Figs. A.2 and A.3 we show a flowchart explaining the behavior of vehicles according to the specific traffic circumstances in which they are involved as well as a small plot representing the different transition states that each vehicle can contemplate during motion.

All vehicles will behave according to five possible states:

1. Basic circulation: the vehicle drives at a constant speed along its path on the road.

2. Reaction to brake: in a normal braking situation (human assistance), the driver will need a small time interval (between 0.5 and $1 \mathrm{~s}$ ) to start to slow down (from the moment the danger is noticed until the brake pedal is pushed).

3. Braking: once the brake pedal is pressed (in human assisted driving) or due to automatic braking in an autonomous driving scheme, the vehicle begins to decelerate so as to reach null speed in the driveway.

4. Vehicle collided: the vehicle could not stop in time to avoid a collision with the vehicle ahead.

5. Vehicle stopped successfully: the vehicle has successfully managed to stop and therefore it avoided the collision. 


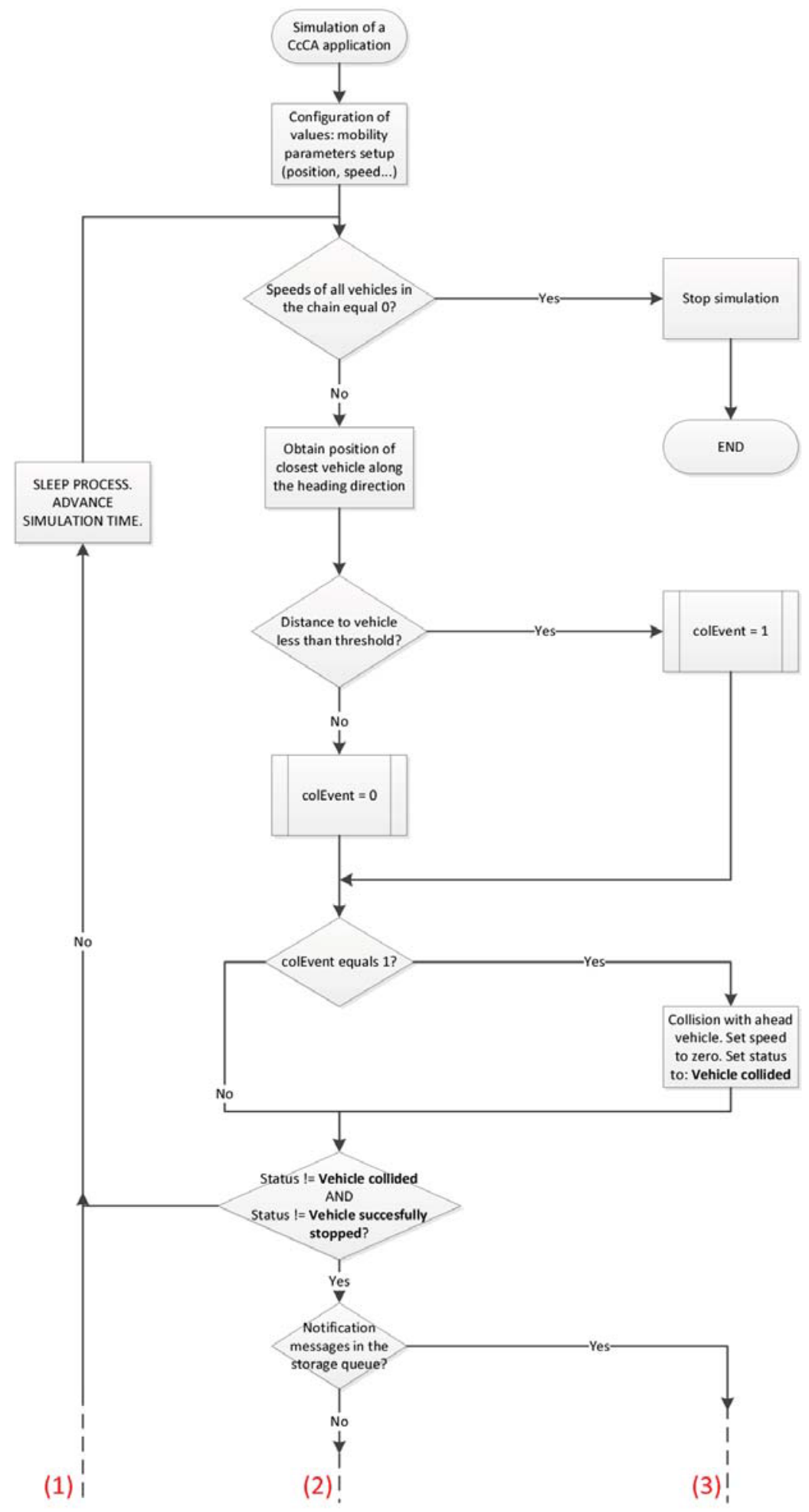

Figure A.2: Communications' module flowchart, part 1 


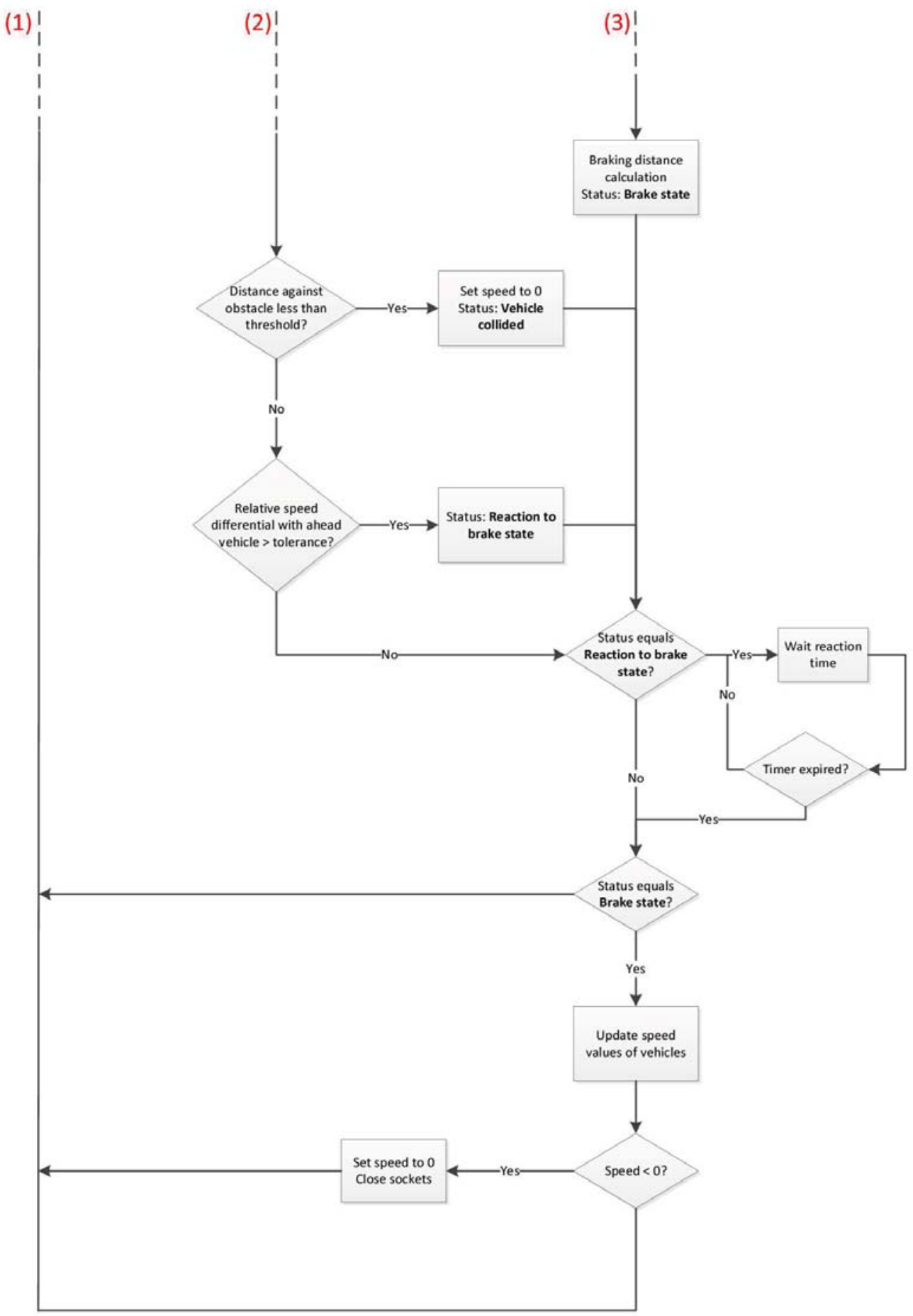

Figure A.3: Communications' module flowchart, part 2 


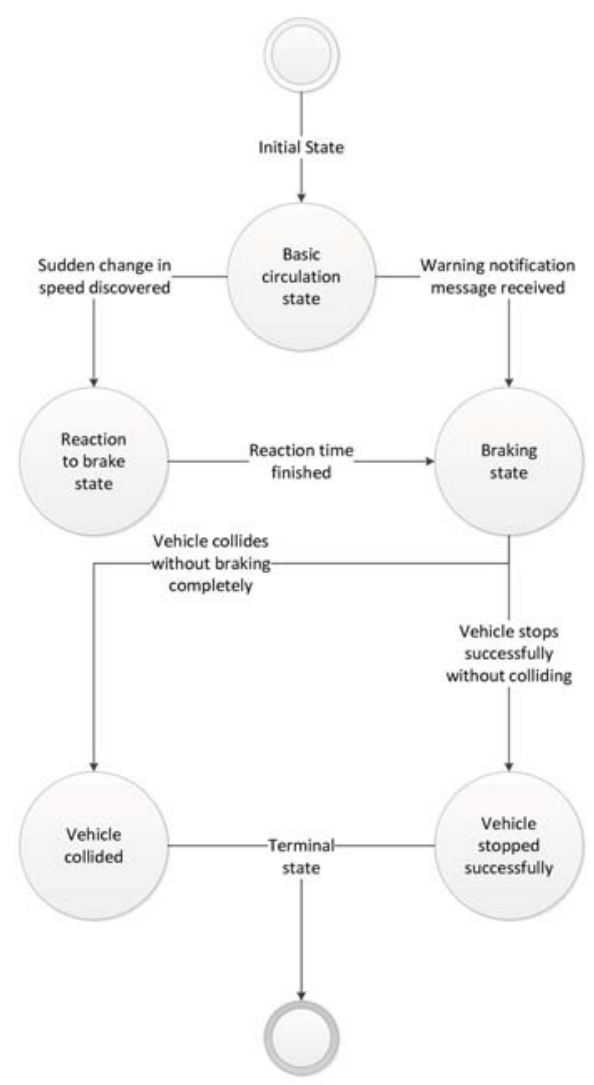

Figure A.4: System's state diagram

As can be seen in the flowchart presented in Figs. A.2 and A.3, first we configure a set of parameters that represents the essential characteristics of mobility of vehicles in this particular scenario: initial driving direction, initial position and initial speed. In our implementation this is achieved by the API implemented in NCTUns for developing user-specific mobility agents, by means of a socket communication scheme that allows sending mobility management commands to the simulation engine.

Then we can observe the logical loop that determines the mobility patterns of the designed model (see the corresponding state's diagram in Fig. A.4). First we evaluate whether the speeds of all vehicles are equal to zero. In this case, the simulation ends to avoid consuming more time. Afterwards it calculates for each vehicle the position of the nearest neighbor in the direction of transit (either ahead or rear). If the distance is less than a certain threshold, we consider this to be collision, so a variable that stores the state of the vehicle (specifically colEvent) is set to 1 ( 0 otherwise). In the following 
step we analyze the value of this variable. If it is equal to 1 , it means that the vehicle is in a state of collision, thus we tell the process governing the mobility of the collided vehicle to stop to save simulation resources. We simply set the vehicle speed to zero and advance the simulation time.

If the vehicle is found in a normal traffic state, that is, neither stopped (by braking successfully without colliding), nor collided, first we evaluate whether there is any message in the mobility queue. Because in our case the only sent message corresponds to alert emergency braking, there is no problem in terms of packet prioritization (as regards the four classes of priority as introduced in the Chapter 2, Part I of this Book). Particularly, all sudden brake warning messages in this implementation contain the following information:

- Position of the collided vehicle that issued the alert: to be used to check whether it is possible to stop in time.

- Timestamp at which the message was sent: to check end to end delay of messages.

- Damaged vehicle identifier: to verify the identity of the vehicle that was involved in the accident.

Should we find such messages in the mobility queue, the receiving vehicle automatically calculates the braking distance that it needs to reach a full standstill stop, according to the equation presented below, obtained from reference (105):

$$
D_{p}=\frac{v_{I} \cdot t_{p}}{3.6}+\frac{v_{I}^{2}}{254\left(i+f_{i}\right)}
$$

Where $v_{I}$ denotes the initial speed in $\mathrm{km} / \mathrm{h}, i$ the road slope coefficient, $t_{p}$ the sum of the perception and the reaction time, and $f_{i}$ representing the longitudinal friction coefficient. The resulting distance (in meters) will be the sum of the distance traveled by the vehicle during the reaction time (first term) and the distance traveled while braking (second term). In our case, we skip the first term in the calculation of $D_{p}$, since we assume the reaction time uniformly distributed between 0.5 and 1 second. As we can see, the relationship between speed and braking distance is squared, implying that two times the speed requires a braking distance multiplied by four. Another important factor to consider is the road friction, if $f_{i}$ is very low due to poor conditions of wheels 


\section{A. OVER THE NCTUNS NETWORK SIMULATOR}

or because of bad weather conditions, the stopping distance may increase considerably, in fact on a icy pavement a car could have a stopping distance of a mile at an average speed of $100 \mathrm{~km} / \mathrm{h}$.

In the case in which the vehicle finds no queued messages in the mobility queue, it checks whether there is any standing obstacle ahead on the road that could become a potential risk of accident. If a vehicle discovers an obstacle blocking the freeway along its path of movement, it will will start to brake. If it collides, it will set the speed to zero, simulating the accident, and send an alert information to following vehicles. Now it will transition to the collision status.

The last part of the algorithm corresponds to a situation where a particular vehicle begins to brake to avoid colliding with the vehicle directly ahead, either because of a too high speed differential, or due to the reception of accident warning notifications. In this case, according to our braking model, we assume that the vehicle decelerates with a speed that decreases linearly with time (constant braking deceleration). Due to the specific implementation of the NCTUns process manager (to switch between different machine processes and advance the simulation time), in our program we have to necessarily set an idle time that the application program allocates for the simulation engine to advance in the simulation time. For this purpose, we have derived a mathematical equation that provides the speed of the vehicle at any time after starting the braking process, regardless of the time duration of the idle period. The expression is:

$$
v\left(t_{k}\right)=v\left(t_{k-1}\right)-\frac{v^{2}\left(t_{k-1}\right)}{2 D_{p}} \cdot\left(t_{k-1}+\frac{S}{2}-t_{\text {stop }}\right) \rho
$$

Where $v\left(t_{k}\right)$ denotes the speed to be set at the current instant, according to the speed at the previous instant $v\left(t_{k-1}\right)$, the previously calculated braking distance $D_{p}$, the sampling interval time of the application (for advancing the simulation time) $S$, the time at which the emergency braking begins $t_{s t o p}$, and the scaling factor of the simulation time $\rho$ (in our program it is expressed in ticks: 1 tick $=100 \mathrm{~ns}$ ).

The derivation of the above expression is explained below. Due to the linear decrease of speed, it will become a value at a future instant which is governed by the expression:

$$
v\left(t_{k}\right)=v\left(t_{k-1}\right)-a \cdot t_{\text {lapse }}
$$


Since the vehicle will need $D_{p}$ meters (according to our mobility model) to fully stop, the following system of equations holds:

$$
\left\{\begin{array}{c}
v_{I}-a \cdot T=0 \\
v_{I} \cdot T-a \cdot \frac{T^{2}}{2}=D_{p}
\end{array}\right.
$$

This system can be understood by realizing that when braking for a time interval of $T$ s (time required to fulfill a complete stop), the car will reach zero speed. Moreover, the second equation expresses the distance $D_{p}$ that is covered during the braking process. If we solve the system of equations we obtain a value for the acceleration that takes the expression:

$$
a=\frac{v_{I}^{2}}{2 D_{p}}
$$

Substituting in the first term we have:

$$
v\left(t_{k}\right)=v\left(t_{k-1}\right)-\frac{v_{I}^{2}}{2 D_{p}} \cdot t_{\text {lapse }}
$$

To give value to $t_{\text {lapse }}$ let us carefully observe Fig. A.5, which represents the evolution of the speed from the moment the vehicle begins to slow down until it completely stops.

As we can see, the simulation engine will dispatch the mobility manager every $S$ time units (ticks), in order to advance the simulation time. Each time the mobility agent is awaken the simulation time will have advanced a time lapse of $S$ ticks, thus needing to conveniently update the speed. However, due to the linear decrease of speed until the vehicle stops, the mobility manager must establish a constant moving speed during the interval when the process sleeps that allows to cover the same distance as if the speed was updated at infinitesimal time periods. Because of that, for obvious reasons we take the speed at the midpoint of each interval of $S$ ticks. This makes $t_{\text {lapse }}$ take the value given by the following expression:

$$
t_{\text {lapse }}=\left(t_{k}+\frac{S}{2}-t_{\text {stop }}\right) \cdot S
$$

Substituting Eq. (A.7) in the second equation of the System (A.4) we will get the value of the speed for successive instants. 


\section{A. OVER THE NCTUNS NETWORK SIMULATOR}

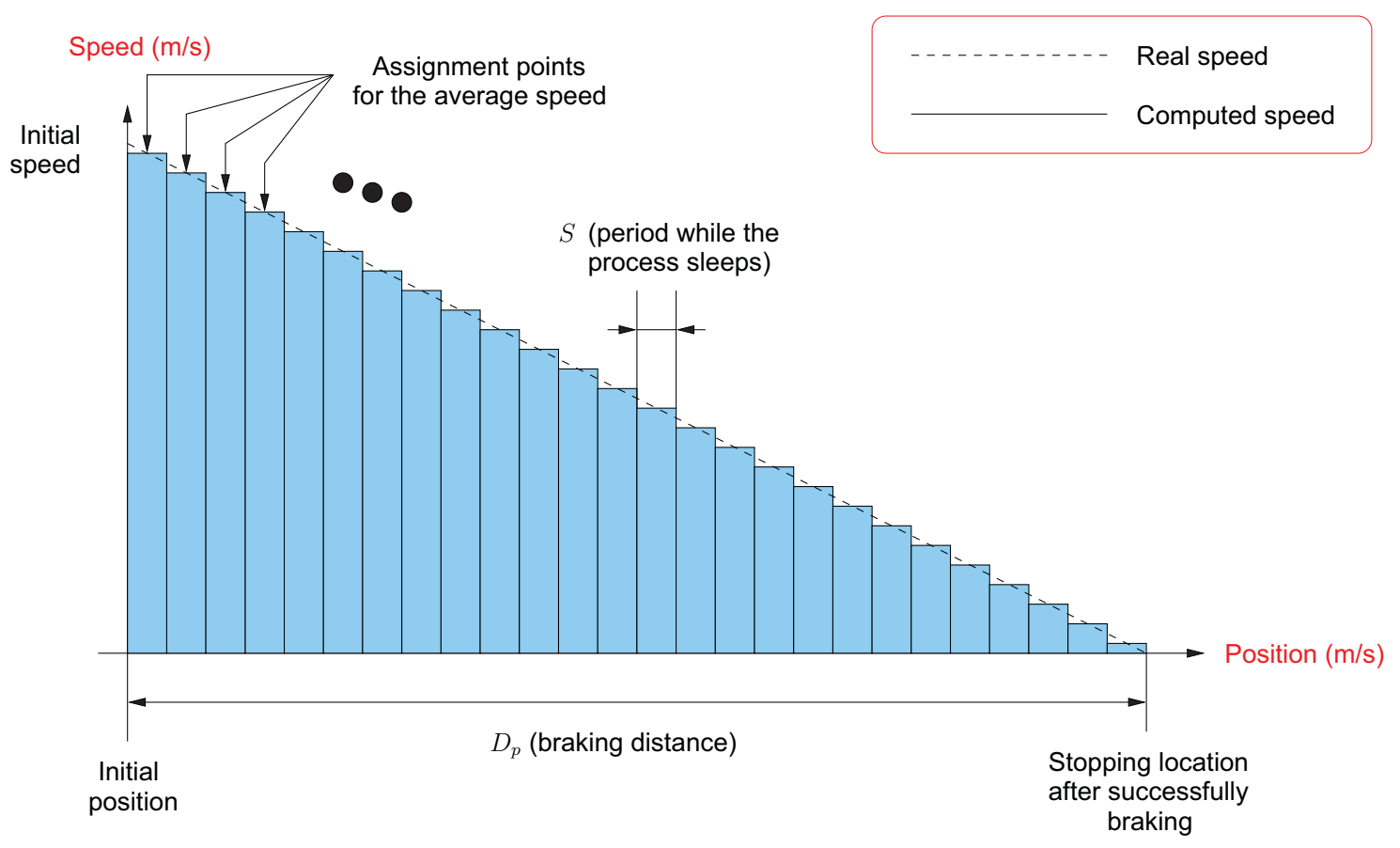

Figure A.5: Sampling period graph for calculating speed during braking

All vehicles will start driving at an average speed that will be one of the representative configuration parameters in simulations. The intervehicular distance will also play an important part in the simulation, since the greater the distance the lower the probability of incurring in chain accidents. Moreover, the transmission power will be decisive on the behavior of vehicles reacting to the reception of warning messages, since it will influence on the end to end delay as well as other performance metrics like throughput. We will also assume for autonomous braking that mobility managers will need an additional processing time of $100 \mathrm{~ms}$ when receiving notifications to start brake, in order to model the necessary processing time of OBUs to execute the braking maneuver.

\section{A.3.2 WSMP Communications' module}

The communications module WSMP (WAVE Short Message Protocol) implements the network layer protocol of the architecture WAVE, using a simplified packet format that reduces overhead for a more efficient packet transmission and processing. This module is already implemented in the NCTUns version we used for simulations, although in the context of our particular application for CcCA, it was necessary to carry out some 
modifications of the code to support bidirectional communications between this protocol and the mobility manager. Particularly, we have defined logical parallel threads to implement bidirectional communication between both modules.

As regards functionality, the WSMP module constantly awaits (when control of the simulation engine is given to the machine processes) the reception of:

- Messages sent by other vehicle reporting a particular issue about traffic safety (specifically, a collision ahead).

- Messages from the mobility management module within the same vehicle, reporting a particular feature of the nearby traffic, because it might have found an obstacle ahead. Therefore, it must inform the other nodes that come behind to conveniently react to this situation.

By implementing these changes in the WSMP module, the system can support bidirectional communication between both the communication and the mobility management modules, allowing us to build our own mobility model supporting active behavior in $\mathrm{CcCA}$ according to the reception of warning messages. 
A. OVER THE NCTUNS NETWORK SIMULATOR 


\section{Appendix B}

\section{Mathematical operations for the stochastic model of Chapter 3}

\section{B.1 Computation of the distance traveled by a vehicle for variable velocity and notification delay}

Let us recall that when velocity and notification delay are not constant the collisions may occur in four different ways: (1) vehicles $C_{i}$ and $C_{i-1}$ have not started to brake; (2) only one of them is braking; (3) both of them are braking; or (4) vehicle $C_{i-1}$ has stopped. Each one of these possibilities results in a different distance to stop, $d_{c_{1}, i}, d_{c_{2}, i}, d_{c_{3}, i}$ and $d_{c_{4}, i}$ respectively that must be weighted by the probability of the event and added to get the average $\overline{l_{i}}$ as in (3.13).

The computation of these distance is as follows:

(1) Collision when the vehicles have not started to brake $\left(d_{c_{1}, i}\right)$. This event may happen if the difference of initial velocities makes the vehicles crash before receiving the warning message ${ }^{1}$.

A time instant $t_{c_{1}, i}\left(s_{i}\right)$ should exist so that

$$
\begin{gathered}
V_{i} t_{c_{1}, i}\left(s_{i}\right)=V_{i-1} t_{c_{1}, i}\left(s_{i}\right)+s_{i}, \\
0 \leq t_{c_{1}, i}\left(s_{i}\right) \leq \min \left\{\delta_{i}, \delta_{i-1}\right\} .
\end{gathered}
$$

\footnotetext{
${ }^{1}$ Let us note that this case implies that the vehicles would collide even if there is no obstacle ahead on the road or the rest of vehicles in the chain are not braking. For instance, a driver notices that his/her actual speed is higher than that of the preceding vehicle but does not reduce it and let his/her car collide. One might think of a situation where bad weather conditions, like a very thick fog, prevent the driver from noticing the risk. In a normal driving situation this case should be highly unlikely but it has to be considered to get a consistent result.
} 


\section{B. MATHEMATICAL OPERATIONS FOR THE STOCHASTIC MODEL OF CHAPTER 3}

Solving (B.1) we obtain

$$
t_{c_{1}, i}\left(s_{i}\right)=\frac{s_{i}}{V_{i}-V_{i-1}} .
$$

Therefore, the distance to stop in this case is $d=V_{i} t_{c_{1}, i}\left(s_{i}\right)$, which is a function of $s_{i}$ (exponentially distributed). Then, the average distance is computed as follows:

$$
d_{c_{1}, i}=\frac{1}{F\left(\sup _{1}\right)-F\left(i n f_{1}\right)} \int_{i n f_{1}}^{\sup _{1}} \frac{V_{i} x}{V_{i}-V_{i-1}} \lambda e^{-\lambda x} d x .
$$

Now, it remains to compute the range of $s_{i}$ where a collision can happen, that is, the integration limits denoted as $i n f_{1}$ and sup $_{1}$ :

- If $V_{i}-V_{i-1} \leq 0$, there is no solution (no collision can occur). Let us define appropriate limits

$$
\begin{aligned}
& i n f_{1}=0, \\
& s u p_{1}=0 .
\end{aligned}
$$

- If $V_{i}-V_{i-1}>0$, then (B.2) holds if and only if $0 \leq s_{i} \leq\left(V_{i}-V_{i-1}\right) \cdot \min \left\{\delta_{i}, \delta_{i-1}\right\}$. Let us define

$$
\begin{aligned}
\text { inf }_{1} & =0, \\
\sup _{1} & =\left(V_{i}-V_{i-1}\right) \cdot \min \left\{\delta_{i}, \delta_{i-1}\right\} .
\end{aligned}
$$

The same procedure is applied for the following cases: the computation of the actual distance traveled, in this case $d=V_{i} t_{c_{1}, i}\left(s_{i}\right)$, where $t_{c_{1}, i}\left(s_{i}\right)=f\left(s_{i}, a, V_{i}, V_{i-1}, \delta_{i}, \delta_{i-1}\right)$, which is multiplied by the exponential pdf and integrated within the appropriate limits, which are also derived.

(2) Collision when only one vehicle is braking $\left(d_{c_{2}, i}\right)$. In this case, the collision event depends on the relative reaction times of the drivers. That is, due to a high reaction time, one of the drivers starts to brake too late.

- If $\delta_{i}=\delta_{i-1}$, then we have to skip to case (3), later in the text, and so let us define

$$
\begin{aligned}
& \text { inf }_{2}=\text { sup }_{1}, \\
& \sup _{2}=\sup _{1} .
\end{aligned}
$$


- If $\delta_{i}<\delta_{i-1}$, then vehicle $C_{i}$ starts to brake before $C_{i-1}$ does.

A time instant $t_{c_{2}, i}\left(s_{i}\right)$ should exist so that

$$
\begin{aligned}
& V_{i} t_{c_{2}, i}\left(s_{i}\right)-\frac{a}{2}\left(t_{c_{2}, i}\left(s_{i}\right)-\delta_{i}\right)^{2}=V_{i-1} t_{c_{2}, i}\left(s_{i}\right)+s_{i}, \\
& \delta_{i} \leq t_{c_{2}, i}\left(s_{i}\right) \leq \delta_{i-1} .
\end{aligned}
$$

Solving this equation, we obtain the following solutions:

$$
\begin{aligned}
t 1_{c_{2}, i}\left(s_{i}\right) & =\frac{V_{i}-V_{i-1}}{a}+\delta_{i}- \\
& -\sqrt{\left(\frac{V_{i}-V_{i-1}}{a}+\delta_{i}\right)^{2}-\delta_{i}^{2}-\frac{2 s_{i}}{a}}, \\
t 2_{c_{2}, i}\left(s_{i}\right) & =\frac{V_{i}-V_{i-1}}{a}+\delta_{i}+ \\
& +\sqrt{\left(\frac{V_{i}-V_{i-1}}{a}+\delta_{i}\right)^{2}-\delta_{i}^{2}-\frac{2 s_{i}}{a}} .
\end{aligned}
$$

The term in the square root is positive if and only if:

$$
s_{i} \leq \delta_{i}\left(V_{i}-V_{i-1}\right)+\frac{\left(V_{i}-V_{i-1}\right)^{2}}{2 a}
$$

It can be proved that (B.12) does not hold for $t 2_{c_{2}, i}\left(s_{i}\right)$, so the only possible solution is $t 1_{c_{2}, i}\left(s_{i}\right)$.

- If $V_{i}-V_{i-1}>a\left(\delta_{i-1}-\delta_{i}\right)$, then (B.12) holds for $t 1_{c_{2}, i}\left(s_{i}\right)$ if and only if

$$
\delta_{i}\left(V_{i}-V_{i-1}\right) \leq s_{i} \leq \delta_{i-1}\left(V_{i}-V_{i-1}\right)-\frac{a}{2}\left(\delta_{i}-\delta_{i-1}\right)^{2} .
$$

Let us define

$$
\begin{aligned}
\inf _{2}= & \delta_{i}\left(V_{i}-V_{i-1}\right), \\
\sup _{2}= & \min \left\{\delta_{i}\left(V_{i}-V_{i-1}\right)+\frac{\left(V_{i}-V_{i-1}\right)^{2}}{2 a},\right. \\
& \left.\delta_{i-1}\left(V_{i}-V_{i-1}\right)-\frac{a}{2}\left(\delta_{i}-\delta_{i-1}\right)^{2}\right\} .
\end{aligned}
$$


- If $0<V_{i}-V_{i-1} \leq a\left(\delta_{i-1}-\delta_{i}\right)$, then (B.12) holds for $t 1_{c_{2}, i}\left(s_{i}\right)$ if and only if

$$
\delta_{i}\left(V_{i}-V_{i-1}\right) \leq s_{i} \leq \delta_{i}\left(V_{i}-V_{i-1}\right)+\frac{\left(V_{i}-V_{i-1}\right)^{2}}{2 a} .
$$

Let us define

$$
\begin{aligned}
& \text { inf }_{2}=\delta_{i}\left(V_{i}-V_{i-1}\right), \\
& \sup _{2}=\delta_{i}\left(V_{i}-V_{i-1}\right)+\frac{\left(V_{i}-V_{i-1}\right)^{2}}{2 a} .
\end{aligned}
$$

- Otherwise, there is no solution, and so let us define

$$
\begin{aligned}
& \inf _{2}=\sup _{1}, \\
& \sup _{2}=\sup _{1} .
\end{aligned}
$$

- If $\delta_{i}>\delta_{i-1}$, then vehicle $C_{i-1}$ starts to brake before $C_{i}$ does.

A time instant $t_{c_{2}, i}\left(s_{i}\right)$ should exist so that

$$
\begin{aligned}
& V_{i} t_{c_{2}, i}\left(s_{i}\right)=V_{i-1} t_{c_{2}, i}\left(s_{i}\right)-\frac{a}{2}\left(t_{c_{2}, i}\left(s_{i}\right)-\delta_{i-1}\right)^{2}+s_{i}, \\
& \delta_{i-1} \leq t_{c_{2}, i}\left(s_{i}\right) \leq \delta_{i} .
\end{aligned}
$$

Solving (B.24), we obtain the following solutions:

$$
\begin{aligned}
& t 1_{c_{2}, i}\left(s_{i}\right)=\frac{V_{i-1}-V_{i}}{a}+\delta_{i-1}- \\
&-\sqrt{\left(\frac{V_{i-1}-V_{i}}{a}+\delta_{i-1}\right)^{2}-\delta_{i-1}^{2}+\frac{2 s_{i}}{a}} \\
& t 2_{c_{2}, i}\left(s_{i}\right)=\frac{V_{i-1}-V_{i}}{a}+\delta_{i-1}+ \\
&+\sqrt{\left(\frac{V_{i-1}-V_{i}}{a}+\delta_{i-1}\right)^{2}-\delta_{i-1}^{2}+\frac{2 s_{i}}{a}}
\end{aligned}
$$

The term in the square root is positive if and only if:

$$
s_{i} \geq \delta_{i-1}\left(V_{i}-V_{i-1}\right)-\frac{\left(V_{i-1}-V_{i}\right)^{2}}{2 a}
$$


It can be proved that (B.25) does not hold for $t 1_{c_{2}, i}\left(s_{i}\right)$, so the only possible solution is $t 2_{c_{2}, i}\left(s_{i}\right)$.

- If $V_{i-1}-V_{i}<0$, then (B.25) holds for $t 2_{c_{2}, i}\left(s_{i}\right)$ if and only if

$$
\delta_{i-1}\left(V_{i}-V_{i-1}\right) \leq s_{i} \leq \delta_{i}\left(V_{i}-V_{i-1}\right)+\frac{a}{2}\left(\delta_{i}-\delta_{i-1}\right)^{2} .
$$

Let us define

$$
\begin{aligned}
\text { inf }_{2} & =\delta_{i-1}\left(V_{i}-V_{i-1}\right), \\
\sup _{2} & =\delta_{i}\left(V_{i}-V_{i-1}\right)+\frac{a}{2}\left(\delta_{i}-\delta_{i-1}\right)^{2} .
\end{aligned}
$$

- If $0 \leq V_{i-1}-V_{i} \leq a\left(\delta_{i}-\delta_{i-1}\right)$, then (B.25) holds for $t 2_{c_{2}, i}\left(s_{i}\right)$ if and only if

$$
\begin{array}{r}
\delta_{i-1}\left(V_{i}-V_{i-1}\right)-\frac{\left(V_{i-1}-V_{i}\right)^{2}}{2 a} \leq s_{i} \leq \\
\leq \delta_{i}\left(V_{i}-V_{i-1}\right)+\frac{a}{2}\left(\delta_{i}-\delta_{i-1}\right)^{2} .
\end{array}
$$

Let us define

$$
\begin{aligned}
& \text { inf }_{2}=\delta_{i-1}\left(V_{i}-V_{i-1}\right)-\frac{\left(V_{i-1}-V_{i}\right)^{2}}{2 a}, \\
& \sup _{2}=\delta_{i}\left(V_{i}-V_{i-1}\right)+\frac{a}{2}\left(\delta_{i}-\delta_{i-1}\right)^{2} .
\end{aligned}
$$

- Otherwise, there is no solution, and so let us define

$$
\begin{aligned}
& i n f_{2}=\sup _{1}, \\
& \sup _{2}=\sup _{1} .
\end{aligned}
$$

(3) Collision when both vehicles are braking $\left(d_{c_{3}, i}\right)$. In this case, both vehicles are aware of the danger and have started to brake but they are not able to avoid the collision, due to their initial speeds and reaction times, and they collide in motion.

A time instant should exist so that

$$
\begin{aligned}
& V_{i} t_{c_{3}, i}\left(s_{i}\right)-\frac{a}{2}\left(t_{c_{3}, i}\left(s_{i}\right)-\delta_{i}\right)^{2}= \\
& =V_{i-1} t_{c_{3}, i}\left(s_{i}\right)-\frac{a}{2}\left(t_{c_{3}, i}\left(s_{i}\right)-\delta_{i-1}\right)^{2}+s_{i}, \\
& \delta_{i} \leq t_{c_{3}, i}\left(s_{i}\right) \leq \frac{V_{i}}{a}+\delta_{i}, \\
& \delta_{i-1} \leq t_{c_{3}, i}\left(s_{i}\right) \leq T_{i-1}\left(\overline{l_{i-1}}\right),
\end{aligned}
$$




\section{B. MATHEMATICAL OPERATIONS FOR THE STOCHASTIC MODEL OF CHAPTER 3}

where $T_{i-1}\left(\overline{l_{i-1}}\right)$ is the time needed by vehicle $C_{i-1}$ to travel the distance $\overline{l_{i-1}}$, and it is calculated by the function:

$$
T_{i}(x)= \begin{cases}\frac{x}{V_{i}}, & \text { if } x \leq V_{i} \delta_{i}, \\ \frac{V_{i}}{a}+\delta_{i}-\sqrt{\frac{2}{a}\left(d_{s, i}-x\right)}, & \text { if } x>V_{i} \delta_{i} .\end{cases}
$$

Solving (B.37), we obtain

$$
t_{c_{3}, i}\left(s_{i}\right)=\frac{s_{i}+\frac{a}{2}\left(\delta_{i}^{2}-\delta_{i-1}^{2}\right)}{\left(V_{i}-V_{i-1}\right)+a\left(\delta_{i}-\delta_{i-1}\right)} .
$$

In order to simplify the notation, we call num $=\frac{a}{2}\left(\delta_{i}^{2}-\delta_{i-1}^{2}\right)$ and den $=\left(V_{i}-\right.$ $\left.V_{i-1}\right)+a\left(\delta_{i}-\delta_{i-1}\right)$

- If den $=0$ there is no solution. Let us define

$$
\begin{aligned}
& \inf _{3}=\sup _{2}, \\
& \sup _{3}=\sup _{2} .
\end{aligned}
$$

- If den > 0, (B.38) and (B.39) hold if and only if

$$
\begin{aligned}
& \text { den } \cdot \max \left\{\delta_{i}, \delta_{i-1}\right\}-\text { num } \leq s_{i} \leq \\
& \leq \text { den } \cdot \min \left\{\frac{V_{i}}{a}+\delta_{i}, T_{i-1}\left(\overline{l_{i-1}}\right)\right\}-\text { num } .
\end{aligned}
$$

And so let us define

$$
\begin{aligned}
\text { inf }_{3} & =\text { den } \cdot \max \left\{\delta_{i}, \delta_{i-1}\right\}-\text { num } \\
\sup _{3} & =\text { den } \cdot \min \left\{\frac{V_{i}}{a}+\delta_{i}, T_{i-1}\left(\overline{l_{i-1}}\right)\right\}-\text { num. }
\end{aligned}
$$

- If den < 0, (B.38) and (B.39) hold if and only if

$$
\begin{aligned}
& \text { den } \cdot \min \left\{\frac{V_{i}}{a}+\delta_{i}, T_{i-1}\left(\overline{l_{i-1}}\right)\right\}-\text { num } \leq s_{i} \leq \\
& \leq \text { den } \cdot \max \left\{\delta_{i}, \delta_{i-1}\right\}-\text { num. }
\end{aligned}
$$

Then, let us define

$$
\begin{aligned}
& \text { inf }_{3}=\text { den } \cdot \min \left\{\frac{V_{i}}{a}+\delta_{i}, T_{i-1}\left(\overline{l_{i-1}}\right)\right\}-\text { num }, \\
& \sup _{3}=\text { den } \cdot \max \left\{\delta_{i}, \delta_{i-1}\right\}-\text { num. }
\end{aligned}
$$


(4) Collision when vehicle $C_{i-1}$ has stopped $\left(d_{c_{4}, i}\right)$. The preceding vehicle has been able to stop safely but a rear collision still occurs.

In this case $s_{i}$ should directly satisfy $s_{i} \leq d_{s, i}-\overline{l_{i-1}}$. We set

$$
\begin{aligned}
\inf _{4} & =\sup _{3} \\
\sup _{4} & =d_{s, i}-\overline{l_{i-1}} .
\end{aligned}
$$

\section{B.2 Computation of the distance traveled by a vehicle for variable velocity and deceleration}

Let us recall that when velocity and deceleration are not constant the collisions may occur in three different ways: (1) vehicles $C_{i}$ and $C_{i-1}$ have not started to brake; (2) both of them are already braking; or (3) vehicle $C_{i-1}$ has stopped, with their respective actual distances to stop $d_{c_{1}, i}, d_{c_{2}, i}$ and $d_{c_{3}, i}$. These distances are computed using the same procedure of Appendix A.

(1) Collision when the vehicles have not started to brake $\left(d_{c_{1}, i}\right)$. This event may happen if the difference of initial velocities makes the vehicles crash before receiving the warning message.

A time instant $t_{c_{1}, i}\left(s_{i}\right)$ should exist so that

$$
\begin{gathered}
V_{i} t_{c_{1}, i}\left(s_{i}\right)=V_{i-1} t_{c_{1}, i}\left(s_{i}\right)+s_{i}, \\
0 \leq t_{c_{1}, i}\left(s_{i}\right) \leq \delta .
\end{gathered}
$$

Solving (B.52) we have

$$
t_{c_{1}, i}\left(s_{i}\right)=\frac{s_{i}}{V_{i}-V_{i-1}} .
$$

Now, it remains to compute the integration limits $i n f_{1}$ and $\sup _{1}$ :

- If $V_{i}-V_{i-1} \leq 0$, there is no solution (no collision can occur). Let us define appropriate limits

$$
\begin{aligned}
& i n f_{1}=0, \\
& \sup _{1}=0 .
\end{aligned}
$$


- If $V_{i}-V_{i-1}>0$, then (B.53) holds if and only if $0 \leq s_{i} \leq \delta\left(V_{i}-V_{i-1}\right)$. Let us define

$$
\begin{aligned}
& \inf _{1}=0, \\
& \sup _{1}=\delta\left(V_{i}-V_{i-1}\right) .
\end{aligned}
$$

(2) Collision when both vehicles are braking $\left(d_{c_{2}, i}\right)$.

A time instant $t_{c_{2}, i}\left(s_{i}\right)$ should exist so that

$$
\begin{aligned}
& V_{i} t_{c_{2}, i}\left(s_{i}\right)-\frac{a_{i}}{2}\left(t_{c_{2}, i}\left(s_{i}\right)-\delta\right)^{2}= \\
& =V_{i-1} t_{c_{2}, i}\left(s_{i}\right)-\frac{a_{i-1}}{2}\left(t_{c_{2}, i}\left(s_{i}\right)-\delta\right)^{2}+s_{i}, \\
& \delta \leq t_{c_{2}, i}\left(s_{i}\right) \leq \min \left\{\frac{V_{i}}{a_{i}}+\delta, T_{i-1}\left(\overline{l_{i-1}}\right)\right\} .
\end{aligned}
$$

In order to simplify the notation, we call $\min =\min \left\{\frac{V_{i}}{a_{i}}+\delta, T_{i-1}\left(\overline{l_{i-1}}\right)\right\}-\delta=$ $\min \left\{\frac{V_{i}}{a_{i}}, T_{i-1}\left(\overline{l_{i-1}}\right)-\delta\right\}$.

- If $a_{i-1}-a_{i}=0$ solving (B.59), we obtain

$$
t_{c_{2}, i}\left(s_{i}\right)=\frac{s_{i}}{V_{i}-V_{i-1}}
$$

- If $V_{i}-V_{i-1} \leq 0$, there is no solution. Let us define

$$
\begin{aligned}
& \inf _{2}=\sup _{1}, \\
& \sup _{2}=\sup _{1} .
\end{aligned}
$$

- If $V_{i}-V_{i-1}>0,($ B.60) holds if and only if

$$
\delta\left(V_{i}-V_{i-1}\right) \leq s_{i} \leq\left(V_{i}-V_{i-1}\right) \cdot \min
$$

And so let us define

$$
\begin{aligned}
& \inf _{2}=\delta\left(V_{i}-V_{i-1}\right), \\
& \sup _{2}=\left(V_{i}-V_{i-1}\right) \cdot \min .
\end{aligned}
$$


- If $a_{i-1}-a_{i} \neq 0$ solving (B.59), we obtain the following solutions

$$
\begin{aligned}
& t 1_{c_{2}, i}\left(s_{i}\right)=\frac{V_{i}-V_{i-1}}{a_{i}-a_{i-1}}+\delta- \\
&-\sqrt{\left(\frac{V_{i}-V_{i-1}}{a_{i}-a_{i-1}}+\delta\right)^{2}-\delta^{2}+\frac{2 s(i)}{a_{i-1}-a_{i}}} \\
& t 2_{c_{2}, i}\left(s_{i}\right)=\frac{V_{i}-V_{i-1}}{a_{i}-a_{i-1}}+\delta+ \\
&+\sqrt{\left(\frac{V_{i}-V_{i-1}}{a_{i}-a_{i-1}}+\delta\right)^{2}-\delta^{2}+\frac{2 s(i)}{a_{i-1}-a_{i}}}
\end{aligned}
$$

The term in the square root is positive if and only if:

$$
\frac{s_{i}}{\left(a_{i-1}-a_{i}\right)} \geq \frac{\delta\left(V_{i}-V_{i-1}\right)}{\left(a_{i-1}-a_{i}\right)}-\frac{\left(V_{i}-V_{i-1}\right)^{2}}{2\left(a_{i-1}-a_{i}\right)^{2}}
$$

First we compute the limits for $t_{1}$ :

If $a_{i-1}-a_{i}>0,\left(\right.$ B.60) does not hold for $t 1_{c_{2}, i}\left(s_{i}\right)$, and so let us define

$$
\begin{aligned}
& \inf _{2 a}=\sup _{1}, \\
& \sup _{2 a}=\sup _{1} .
\end{aligned}
$$

If $a_{i-1}-a_{i}<0$, then

- If $\frac{V_{i}-V_{i-1}}{a_{i}-a_{i-1}} \leq \min$, then (B.60) holds for $t 1_{c_{2}, i}\left(s_{i}\right)$ if and only if

$$
\delta\left(V_{i}-V_{i-1}\right) \leq s_{i} \leq \delta\left(V_{i}-V_{i-1}\right)-\frac{\left(V_{i}-V_{i-1}\right)^{2}}{2\left(a_{i-1}-a_{i}\right)} .
$$

Let us define

$$
\begin{aligned}
\inf _{2 a} & =\delta\left(V_{i}-V_{i-1}\right), \\
\sup _{2 a} & =\delta\left(V_{i}-V_{i-1}\right)-\frac{\left(V_{i}-V_{i-1}\right)^{2}}{2\left(a_{i-1}-a_{i}\right)} .
\end{aligned}
$$


- If $\frac{V_{i}-V_{i-1}}{a_{i}-a_{i-1}}>\min$, then (B.60) holds for $t 1_{c_{2}, i}\left(s_{i}\right)$ if and only if

$$
\begin{aligned}
& \delta\left(V_{i}-V_{i-1}\right) \leq s_{i} \leq \frac{a_{i-1}-a_{i}}{2} \cdot \min ^{2}+ \\
& +\left(V_{i}-V_{i-1}\right) \cdot \min +\delta\left(V_{i}-V_{i-1}\right) .
\end{aligned}
$$

Let us define

$$
\begin{aligned}
\inf _{2 a}= & \delta\left(V_{i}-V_{i-1}\right), \\
\sup _{2 a}= & \min \left\{\delta\left(V_{i}-V_{i-1}\right)-\frac{\left(V_{i}-V_{i-1}\right)^{2}}{2\left(a_{i-1}-a_{i}\right)}\right. \\
& \frac{a_{i-1}-a_{i}}{2} m_{i n}{ }^{2}+\left(V_{i}-V_{i-1}\right) \cdot \min + \\
& \left.+\delta\left(V_{i}-V_{i-1}\right)\right\} .
\end{aligned}
$$

It remains to compute the limits for $t 2_{c_{2}, i}\left(s_{i}\right)$ :

If $\frac{V_{i}-V_{i-1}}{a_{i}-a_{i-1}}>\min ,\left(\right.$ B.60) does not hold for $t 2_{c_{2}, i}\left(s_{i}\right)$, and so let us define

$$
\begin{aligned}
& \inf _{2 b}=\sup _{2 a}, \\
& \sup _{2 b}=\sup _{2 a} .
\end{aligned}
$$

If $\frac{V_{i}-V_{i-1}}{a_{i}-a_{i-1}} \leq \min$, then

- If $a_{i-1}-a_{i}>0$ and $V_{i}-V_{i-1}>0$, then (B.60) holds for $t 2_{c_{2}, i}\left(s_{i}\right)$ if and only if

$$
\begin{aligned}
& \delta\left(V_{i}-V_{i-1}\right) \leq s_{i} \leq \frac{a_{i-1}-a_{i}}{2} \cdot \min ^{2}+ \\
& +\left(V_{i}-V_{i-1}\right) \cdot \min +\delta\left(V_{i}-V_{i-1}\right) .
\end{aligned}
$$

Let us define

$$
\begin{aligned}
\inf _{2 b} & =\delta\left(V_{i}-V_{i-1}\right), \\
\sup _{2 b} & =\frac{a_{i-1}-a_{i}}{2} \cdot \min ^{2}+ \\
& +\left(V_{i}-V_{i-1}\right) \min +\delta\left(V_{i}-V_{i-1}\right) .
\end{aligned}
$$


- If $a_{i-1}-a_{i}>0$ and $V_{i}-V_{i-1}<0$, then (B.60) holds for $t 2_{c_{2}, i}\left(s_{i}\right)$ if and only if

$$
\begin{aligned}
& \delta\left(V_{i}-V_{i-1}\right)-\frac{\left(V_{i}-V_{i-1}\right)^{2}}{2\left(a_{i-1}-a_{i}\right)} \leq s_{i} \leq \\
& \leq \frac{a_{i-1}-a_{i}}{2} \cdot \min ^{2}+\left(V_{i}-V_{i-1}\right) \cdot \min + \\
& +\delta\left(V_{i}-V_{i-1}\right) .
\end{aligned}
$$

Let us define

$$
\begin{aligned}
& \inf _{2 b}=\delta\left(V_{i}-V_{i-1}\right)-\frac{\left(V_{i}-V_{i-1}\right)^{2}}{2\left(a_{i-1}-a_{i}\right)}, \\
& \sup _{2 b}=\frac{a_{i-1}-a_{i}}{2} \cdot m_{i n}^{2}+ \\
& \quad+\left(V_{i}-V_{i-1}\right) \min +\delta\left(V_{i}-V_{i-1}\right) .
\end{aligned}
$$

- If $a_{i-1}-a_{i}<0$ and $V_{i}-V_{i-1}>0$, then (B.60) holds for $t 2_{c_{2}, i}\left(s_{i}\right)$ if and only if

$$
\begin{aligned}
& \frac{a_{i-1}-a_{i}}{2} \cdot \min ^{2}+\left(V_{i}-V_{i-1}\right) \cdot \min + \\
& +\delta\left(V_{i}-V_{i-1}\right) \leq s_{i} \leq \delta\left(V_{i}-V_{i-1}\right)- \\
& -\frac{\left(V_{i}-V_{i-1}\right)^{2}}{2\left(a_{i-1}-a_{i}\right)}
\end{aligned}
$$

Let us define

$$
\begin{aligned}
\inf _{2 b} & =\frac{a_{i-1}-a_{i}}{2} \cdot \min ^{2}+ \\
& +\left(V_{i}-V_{i-1}\right) \min +\delta\left(V_{i}-V_{i-1}\right), \\
\sup _{2 b} & =\delta\left(V_{i}-V_{i-1}\right)-\frac{\left(V_{i}-V_{i-1}\right)^{2}}{2\left(a_{i-1}-a_{i}\right)} .
\end{aligned}
$$

- Otherwise, there is no solution, and so let us define

$$
\begin{aligned}
& \inf _{2 b}=\sup _{2 a}, \\
& \sup _{2 b}=\sup _{2 a} .
\end{aligned}
$$

(3) Collision when vehicle $C_{i-1}$ has stopped $\left(d_{c_{3}, i}\right)$. The preceding vehicle has been able to stop safely but a rear-end collision occurs.

In this case $s_{i}$ should directly satisfy that $s_{i} \leq d_{s, i}-\overline{l_{i-1}}$. We set

$$
\begin{aligned}
& \inf _{3}=\sup _{2} \\
& \sup _{3}=d_{s, i}-\overline{l_{i-1}} .
\end{aligned}
$$


B. MATHEMATICAL OPERATIONS FOR THE STOCHASTIC MODEL OF CHAPTER 3 


\section{Appendix C}

\section{Mathematical operations on Chapter 6}

\section{C.1 Derivation of Eq. (6.42)}

When calculating the partial derivative $\frac{\partial \phi_{m, \beta}(k)}{\partial a_{p}(s)}$, Eq. (6.42), we can find four different situations, depending on how the associated entity $p$ is surrounded (Fig. C.1): a) An obstacle on each side. b) One obstacle on the left and one vehicle on the right. c) One vehicle on the left and one obstacle on the right. d) A vehicle on each side.

If we take Eq. (6.40) and particularize for the first case, we get:

\section{Case a)}

$$
\begin{aligned}
\frac{\partial \phi_{m, \beta}(k)}{\partial a_{p}(s)} & =-2\left(z_{\beta+1}-x_{p}(k)-C_{3}\right) \frac{\partial x_{p}(k)}{\partial a_{p}(s)}+ \\
& +2\left(x_{p}(k)-z_{\beta}-C_{2}\right) \frac{\partial x_{p}(k)}{\partial a_{p}(s)}
\end{aligned}
$$

If we do the same for the other three cases, we have:

Case b)

$$
\begin{aligned}
\frac{\partial \phi_{m, \beta}(k)}{\partial a_{p}(s)} & =-2\left(x_{p+1}(k)-x_{p}(k)-C_{4}(p+1)\right) \frac{\partial x_{p}(k)}{\partial a_{p}(s)}+ \\
& +2\left(x_{p}(k)-z_{\beta}-C_{2}\right) \frac{\partial x_{p}(k)}{\partial a_{p}(s)}
\end{aligned}
$$



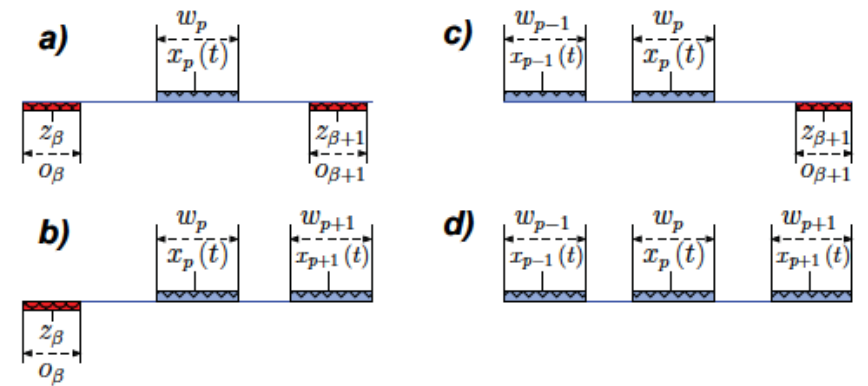

Figure C.1: Particularization cases to obtain the partial derivative $\frac{\partial \phi_{m, \beta}(k)}{\partial a_{p}(s)}$

Case c)

$$
\begin{aligned}
\frac{\partial \phi_{m, \beta}(k)}{\partial a_{p}(s)} & =-2\left(z_{\beta+1}-x_{p}(k)-C_{3}\right) \frac{\partial x_{p}(k)}{\partial a_{p}(s)}+ \\
& +2\left(x_{p}(k)-x_{p-1}(k)-C_{4}(p)\right) \frac{\partial x_{p}(k)}{\partial a_{p}(s)}
\end{aligned}
$$

Case d)

$$
\begin{aligned}
\frac{\partial \phi_{m, \beta}(k)}{\partial a_{p}(s)} & =-2\left(x_{p+1}(k)-x_{p}(k)-C_{4}(p+1)\right) \frac{\partial x_{p}(k)}{\partial a_{p}(s)}+ \\
& +2\left(x_{p}(k)-x_{p-1}(k)-C_{4}(p)\right) \frac{\partial x_{p}(k)}{\partial a_{p}(s)}
\end{aligned}
$$

From Eqs. (C.1-C.4) it is easy to see that we can express Eq. (6.40) in a compact form, Expression (6.42), by expanding as seen in Eq. (6.5) using the equivalences of (6.43), and the result in Eq. (6.41).

\section{C.2 Constraints manipulation for the optimization proce- dure}

In this section we focus on the mathematical manipulations that are necessary to carry out on the constraining relations as regards the Modified Gradient Projection algorithm (MGP, in Chapter 6, Section 6.3.4). We will use matrix notation to express the 
constraints in Eqs. (6.9-6.10). Performing subtle manipulations of the restrictions in discretized form, Eqs. (6.11-C.5) we can rewrite them as:

$$
\begin{aligned}
& \begin{array}{c}
a_{j}(k)+c\left(v_{I}\right) \geq 0 \\
-a_{j}(k)+c\left(v_{I}\right) \geq 0
\end{array} \quad k \in\{0, \ldots, N\}, j \in\{1, \ldots, L\} \\
& x_{1}(k)-\frac{w_{1}}{2} \geq 0 \quad k \in\{0, \ldots, N\} \\
& x_{j+1}(k)-x_{j}(k)-\left(\frac{w_{j}+w_{j+1}}{2}\right) \geq 0 \\
& k \in\{0, \ldots, N\}, j \in\{1, \ldots, L-1\} \\
& x_{L}(k)+\left(W-\frac{w_{L}}{2}\right) \geq 0 \quad k \in\{0, \ldots, N\}
\end{aligned}
$$

Since all the constraints must be expressed as a function of the instantaneous acceleration, we will rewrite them following the next matrix notation:

$$
\begin{gathered}
\qquad\left(\begin{array}{c}
\mathbf{A}_{1} \\
\mathbf{A}_{2} \\
\mathbf{X}
\end{array}\right) \times \mathbf{a}+\left(\begin{array}{l}
\mathbf{V}_{\mathbf{1}} \\
\mathbf{V}_{\mathbf{2}} \\
\mathbf{V}_{\mathbf{3}}
\end{array}\right) \geq\left(\begin{array}{l}
\mathbf{0} \\
\mathbf{0} \\
\mathbf{0}
\end{array}\right) \\
\text { where } \quad \mathbf{A}_{1}, \mathbf{A}_{2} \in \mathbb{R}^{\mathrm{L}(\mathrm{N}+1) \times \mathrm{L}(\mathrm{N}+1)}, \mathbf{V}_{1}, \mathbf{V}_{2} \in \mathbb{R}^{\mathrm{L}(\mathrm{N}+1)} \\
\mathbf{X} \in \mathbb{R}^{\mathrm{L}(\mathrm{N}+2) \times \mathrm{L}(\mathrm{N}+1)}, \mathbf{V}_{3} \in \mathbb{R}^{\mathrm{L}(\mathrm{N}+2)} \\
\mathbf{a} \in \mathbb{R}^{\mathrm{L}(\mathrm{N}+1)}
\end{gathered}
$$

Now let's start defining the matrices $\mathbf{A}_{1}, \mathbf{A}_{2}$ and $\mathbf{X}$, and their respectives $\mathbf{V}$ matrices. $\mathbf{A}_{1}$ and $\mathbf{A}_{2}$ are very easy to define:

$$
\begin{array}{cc}
\mathbf{A}_{1}=\mathbf{I}_{\mathrm{L}(\mathrm{N}+1)} & \mathbf{V}_{1}=\left(\mathbf{c}\left(v_{I}\right)\right) \\
\mathbf{A}_{2}=-\mathbf{I}_{\mathrm{L}(\mathrm{N}+1)} & \mathbf{V}_{2}=\left(\mathbf{c}\left(v_{I}\right)\right)
\end{array}
$$

where $\mathbf{I}_{\mathrm{L}(\mathrm{N}+1)}$ is the $L(N+1)$ identity matrix. $\quad \mathbf{V}_{\mathbf{1}}$ and $\mathbf{V}_{\mathbf{2}}$ are two vectors of length $L(N+1)$ with all the elements equal to the absolute value of the maximum lateral acceleration.

Now, to express position constraints in matrix notation, we will write $\mathbf{X}$ as a block matrix, where each block determines the corresponding coefficients of the instantaneous 
accelerations of vehicle $j$ for the $(N+1)$ time instants in which the trajectory is divided. Each constraint will be referred to the specific row where its respective block is located.

$$
\begin{gathered}
\mathbf{X}=\left(\begin{array}{cccccc}
\mathbf{B}_{1,1} & \mathbf{0} & \mathbf{0} & \cdots & \mathbf{0} & \mathbf{0} \\
\mathbf{B}_{2,1} & \mathbf{B}_{2,2} & \mathbf{0} & \cdots & \mathbf{0} & \mathbf{0} \\
\mathbf{0} & \mathbf{B}_{3,2} & \mathbf{B}_{3,3} & \cdots & \mathbf{0} & \mathbf{0} \\
\vdots & \vdots & \vdots & \ddots & \vdots & \vdots \\
\mathbf{0} & \mathbf{0} & \mathbf{0} & \cdots & \mathbf{B}_{L, L-1} & \mathbf{B}_{L, L} \\
\mathbf{0} & \mathbf{0} & \mathbf{0} & \cdots & \mathbf{0} & \mathbf{B}_{L+1, L}
\end{array}\right) \\
\quad \mathbf{X} \in \mathbb{R}^{(L+1)(N+1) \times L N}
\end{gathered}
$$

Mapping each block of the matrix in Eq.(C.12) to the constraints in (C.5), we finally obtain the matrix structure for the $\mathbf{B}$ sub-matrices. For $\mathbf{B}_{\mathbf{1 , 1}}$ we have:

$$
\mathbf{B}_{1,1}=\left(\begin{array}{ccccccc}
0 & 0 & 0 & \cdots & 0 & 0 & 0 \\
0 & 0 & 0 & \cdots & 0 & 0 & 0 \\
\frac{1}{\xi_{1}} & 0 & 0 & \cdots & 0 & 0 & 0 \\
\frac{2}{\xi_{2}} & \frac{1}{\xi_{2}} & 0 & \cdots & 0 & 0 & 0 \\
\vdots & \vdots & \vdots & \ddots & \vdots & \vdots & \vdots \\
\frac{N-2}{\xi_{N-2}} & \frac{N-3}{\xi_{N-2}} & \frac{N-4}{\xi_{N-2}} & \cdots & \frac{1}{\xi_{N-2}} & 0 & 0 \\
\frac{N-1}{\xi_{N-1}} & \frac{N-2}{\xi_{N-1}} & \frac{N-3}{\xi_{N-1}} & \cdots & \frac{2}{\xi_{N-1}} & \frac{1}{\xi_{N-1}} & 0
\end{array}\right)
$$

For the rest:

$$
\begin{gathered}
\mathbf{B}_{\mathrm{L}+1, \mathrm{~L}}=-\mathbf{B}_{1,1} \\
\mathbf{B}_{\mathrm{n}, \mathrm{n}}=\frac{\mathbf{B}_{1,1}}{\sqrt{2}} \quad n \in\{2, \ldots, L\} \\
\mathbf{B}_{\mathrm{n}, \mathrm{n}-1}=\frac{\mathbf{B}_{L+1, L}}{\sqrt{2}} \quad n \in\{2, \ldots, L\}
\end{gathered}
$$

where

$$
\xi_{k}=\frac{\sqrt{k(k+1)(2 k+1)}}{\sqrt{6}}
$$


If we proceed now to define mathematically $\mathbf{V}_{\mathbf{3}}$ in subvectors, we will have a generic matrix as shown in Eq.(C.20):

$$
\mathbf{V}_{\mathbf{3}}=\left(\begin{array}{c}
\nu_{1} \\
\nu_{2} \\
\nu_{3} \\
\vdots \\
\nu_{L} \\
\nu_{L+1}
\end{array}\right) \in \mathbb{R}^{(L+1)(N+1)}
$$

where

$$
\nu_{j}=\frac{\sqrt{2}}{4 \Delta^{2} t}\left(\begin{array}{c}
2\left(\chi_{0 j}\right)-\omega_{j} \\
2\left(\chi_{0 j}+\vartheta_{0 j} \Delta t\right)-\omega_{j} \\
\frac{2\left(\chi_{0 j}+2 \vartheta_{0 j} \Delta t\right)-\omega_{j}}{\xi_{1}} \\
\frac{2\left(\chi_{0 j}+3 \vartheta_{0 j} \Delta t\right)-\omega_{j}}{\xi_{2}} \\
\vdots \\
\frac{2\left(\chi_{0 j}+(N-1) \vartheta_{0 j} \Delta t\right)-\omega_{j}}{\xi_{N-2}} \\
\frac{2\left(\chi_{0 j}+N \vartheta_{0 j} \Delta t\right)-\omega_{j}}{\xi_{N-1}}
\end{array}\right) \in \mathbb{R}^{N+1}
$$

with

$$
\left.\begin{array}{c}
\chi_{0 j}=X_{0 j}-X_{0 j-1} \\
\vartheta_{0 j}=V_{0 j}-V_{0 j-1} \\
\omega_{j}=w_{j}+w_{j-1}
\end{array}\right\} j \in\{2, \ldots, L\}
$$

For the cases where $i=1$ and $i=L$ we have:

$$
\nu_{1}=\sqrt{2} \nu_{j=1} \quad \nu_{L+1}=\sqrt{2} \nu_{j=L+1}
$$

where

$$
\begin{aligned}
\chi_{01} & =X_{01} \\
\vartheta_{01} & =V_{01} \\
\omega_{1} & =w_{1}
\end{aligned}
$$

and

$$
\begin{gathered}
\chi_{0, L+1}=-X_{0, L} \\
\vartheta_{0, L+1}=-V_{0, L} \\
\omega_{L+1}=-2 W+w_{L}
\end{gathered}
$$




\section{MATHEMATICAL OPERATIONS ON CHAPTER 6}

To derive the matrix (C.12) we need to map each of the $(L+1)$ position constraints of (C.6-C.8) to the B blocks. Among the $(L+1)$ restrictions, there are two related to the positions of either the first (C.6) and the last car (C.8), whose main goal is to limit their positions to the feasible dimensions of the road, i.e. between $x=0$ and $x=W$. The rest of the constraints (C.7) regards the non-overlapping condition that the displacement of vehicles must obey. We will start this derivation by expanding (C.6) using the acceleration-dependent expression of the position on the projected axis in Eq. (6.5).

Expanding (C.6) for all time instants $(k \in\{0, \ldots, N\})$ we get:

$$
X_{01}+k \Delta t \cdot V_{01}+\Delta^{2} t \cdot \sum_{l=0}^{k-2}(k-l-1) \cdot a_{1}(l)-\frac{w_{1}}{2} \geq 0
$$

Rearranging, we group terms in Eq. (C.26) as shown in Eq. (C.9):

$$
\sum_{l=0}^{k-2}(k-l-1) \cdot a_{1}(l)+\left(\frac{X_{01}+k \Delta t \cdot V_{01}-\frac{w_{1}}{2}}{\Delta^{2} t}\right) \geq 0
$$

Before we continue, it is necessary to normalize the coefficients of the instantaneous accelerations (for all $n \in\{1, \ldots, L-1\}$ and $k \in\{0, \ldots, N\}$ ), so that the square of their sum adds to 1 . If we have a look at the term multiplying the accelerations, it is straightforward that applying the coefficient normalization gives the typical square sum of integers factor:

$$
\xi_{k}^{2}=\sum_{l=1}^{k} l^{2}=\frac{k(k+1)(2 k+1)}{6}
$$

Taking the square root of the normalization factor $\xi_{k}^{2}$ and dividing all the terms in (C.27) by $\xi_{k}$, we finally obtain:

$$
\sum_{l=0}^{k-2} \frac{(k-l-1)}{\xi_{k-1}} \cdot a_{1}(l)+\left(\frac{X_{01}+k \Delta t \cdot V_{01}-\frac{w_{1}}{2}}{\xi_{k-1} \Delta^{2} t}\right) \geq 0
$$

From Eq. (C.29) it is easy to map the structure of the matrix block $\mathbf{B}_{1,1}$ in (C.14). It is also easy to prove the correspondence of the independent term of the Ineq. (C.27) with $\nu_{1}$ in $\mathbf{V}_{\mathbf{3}}$ when particularizing Eq. (C.21) for $j=1$. 
The other constraint that limits the position of the last vehicle within the dimensions of the road was introduced in (C.8). Operating with it in the same way as we did for first constraint, we get:

$$
\begin{aligned}
& -\sum_{l=0}^{k-2} \frac{(k-l-1)}{\xi_{k-1}} \cdot a_{L}(l)+ \\
& +\left(\frac{-X_{0 L}-k \Delta t \cdot V_{0 L}+W-\frac{w_{L}}{2}}{\xi_{k-1} \Delta^{2} t}\right) \geq 0
\end{aligned}
$$

From Eq. (C.30) it is easy to see that the coefficients of the acceleration for this case obey $\mathbf{B}_{\mathrm{L}+1, \mathrm{~L}}=-\mathbf{B}_{1,1}$ as was stated in Eq. (C.17). On the other hand, the correspondence between $\nu_{L+1}$ in $\mathbf{V}_{\mathbf{3}}$ and the particularization of $\nu_{j}$ for $j=L+1$ in Vector (C.20) (by using Equalities in (C.25)) is straightforward.

If we focus now on the rest of intermediate, intervehicle non-overlapping constraints (C.7), we have to map:

$$
\begin{gathered}
\mathbf{x}_{n+1}(k)-\mathbf{x}_{n}(k)-\left(\frac{w_{n}+w_{n+1}}{2}\right) \geq 0 \\
n \in\{1, L-1\}, k \in\{0, N\}
\end{gathered}
$$

Using Expressions in (C.23), if we expand Eq. (C.31) using the instantaneous accelerations as independent variables, we get:

$$
\begin{aligned}
& \chi_{0 n+1}+k \Delta t \cdot \vartheta_{0 n+1}+ \\
& +\Delta^{2} t \cdot \sum_{l=0}^{k-2}(k-l-1) \cdot\left(a_{n+1}(l)-a_{n}(l)\right)+ \\
& +\left(\frac{-w_{n+1}-w_{n}}{2}\right) \geq 0
\end{aligned}
$$

Rearranging terms in Eq. (C.32) and isolating the acceleration variables:

$$
\begin{aligned}
& \sum_{l=0}^{k-2}(k-l-1) \cdot\left(a_{n+1}(l)-a_{n}(l)\right)+ \\
& +\left(\frac{\chi_{0 n+1}+k \Delta t \cdot \vartheta_{0 n+1}+\left(\frac{-w_{n+1}-w_{n}}{2}\right)}{\Delta^{2} t}\right) \geq 0
\end{aligned}
$$


We see from the inequalities in (C.33) that to normalize in this case we have the coefficients of the expansion of both $a_{n+1}$ and $a_{n}$, which implies two times the sum of the series in (C.28). We obtain then:

$$
\begin{aligned}
& \sum_{l=0}^{k-2} \frac{(k-l-1)}{\sqrt{2} \xi_{k-1}} \cdot\left(a_{n+1}(l)-a_{n}(l)\right)+ \\
& +\left(\frac{\chi_{0 n+1}+k \Delta t \cdot \vartheta_{0 n+1}+\left(\frac{-w_{n+1}-w_{n}}{2}\right)}{\sqrt{2} \xi_{k-1} \Delta^{2} t}\right) \geq 0
\end{aligned}
$$

Thus, when mapping the inequalities in (C.34) to the $\mathbf{B}$ matrix blocks we obtain what we saw in (C.17) and (C.18), i.e. $\mathbf{B}_{\mathrm{n}, \mathrm{n}}$ and $\mathbf{B}_{\mathrm{n}, \mathrm{n}-1}$. Finally, it is straightforward to prove the correspondence between $\nu_{j}$ for $j \in\{2, \ldots, L\}$ in Vector $\mathbf{V}_{\mathbf{3}}$ (C.20) and the independent terms (between parenthesis) in (C.34) by particularizing for this case. 


\section{Appendix D}

\section{Trajectory optimization under Gaussian Noise disturbances}

\section{D.1 Introduction}

In Chapter 6 we dealt with the cooperative optimization of collision avoidance trajectories for multiple vehicles according to different possible performance criteria. Needless to say, once the optimum evasive maneuvers are determined (here for the sake of simplicity, we disregard the computational time to obtain the trajectories), the vehicles obviously start running the calculated paths. In real cases, the vehicles will have to face undesirable phenomena during the maneuvers' execution which could perturb the previously calculated optimum trajectories and make it divert from the previously calculated paths. This is what in the literature is usually characterized by Random Noise Disturbances (114).

In this Appendix we will extend the initial System of Eqs. (6.1) of Chapter 6 describing vehicular motion to accomplish for this variability in the execution of the maneuvers. Since we are only focusing on the influence of noise on trajectories, we reduce the complexity of the problem to the Scenario shown in Fig. D.1, where there are no intermediate obstacles $(M=0)$, and the size of the crash barriers is reduced to zero. We will also consider the vehicle to have zero length $\left(w_{1}=0 \mathrm{~m}\right)$. The System of Eqs. 6.1 now takes the form (without corrupting noise):

$$
\left\{\begin{array}{l}
\dot{x}_{1}(t)=v_{1}(t) \\
\dot{v}_{1}(t)=a_{1}(t)
\end{array}\right.
$$




\section{TRAJECTORY OPTIMIZATION UNDER GAUSSIAN NOISE DISTURBANCES}

\section{D.2 Performance criteria and formulation extension}

The two main objectives contemplated in this Appendix, which the trajectory should meet during the $t_{f} \mathrm{~s}$, are: i) The maximization of the lateral distance (minimization of the lateral distance variance), and ii) Minimization of the lateral speed at the end of the maneuver. Only two specific particularizations of the performance measure seen in Eq. 6.24 of Chapter 6 for these two objectives are evaluated here ${ }^{1}$ :

- Maximization of the lateral distance and minimization of the final speed at $t_{f}$. This performance metric will be referred to as $J_{D 1}$, and will correspond to the combination of weights $\theta_{x}=1, \theta_{v}=1$ and $\theta_{a}=0$, taking the expression $(L=1$, $\left.M=0, w_{1}=0 \mathrm{~m}\right)$ :

$$
J_{D 1}=x_{1}^{2}\left(t_{f}\right)+\left(W-x_{1}\left(t_{f}\right)\right)^{2}+v_{1}\left(t_{f}\right)
$$

- Maximization of the lateral distance. In this case we will refer to this performance measure as $J_{D 2}$, and it will represent the particularization of Eq. 6.24 for the set of weights $\theta_{x}=1, \theta_{v}=0$ and $\theta_{a}=0\left(L=1, M=0, w_{1}=0 \mathrm{~m}\right)$ :

$$
J_{D 2}=x_{1}^{2}\left(t_{f}\right)+\left(W-x_{1}\left(t_{f}\right)\right)^{2}
$$

We will model the trajectory corrupting phenomena by assuming that an additive Gaussian noise process produces this variability by influencing:

- The shape of the traced path due to possible deviations from the optimum course, see Subsection D.2.1

- The measurement that sensors do on the position and speed at a fixed time $t$, see Subsection D.2.2

Kalman Filter theory has been proven to be a very reasonable option for state estimation and path reconstruction under the aforementioned circumstances. Moreover, it is the core technology which implements basic car motion on autonomous vehicles,

\footnotetext{
${ }^{1}$ Taking into account that we deal here with only $L=1$ vehicle and $M=0$ intermediate obstacles, we can take the formulation for the main performance measure of Section 6.3.2 in Chapter 6, due to not losing differentiability.
} 


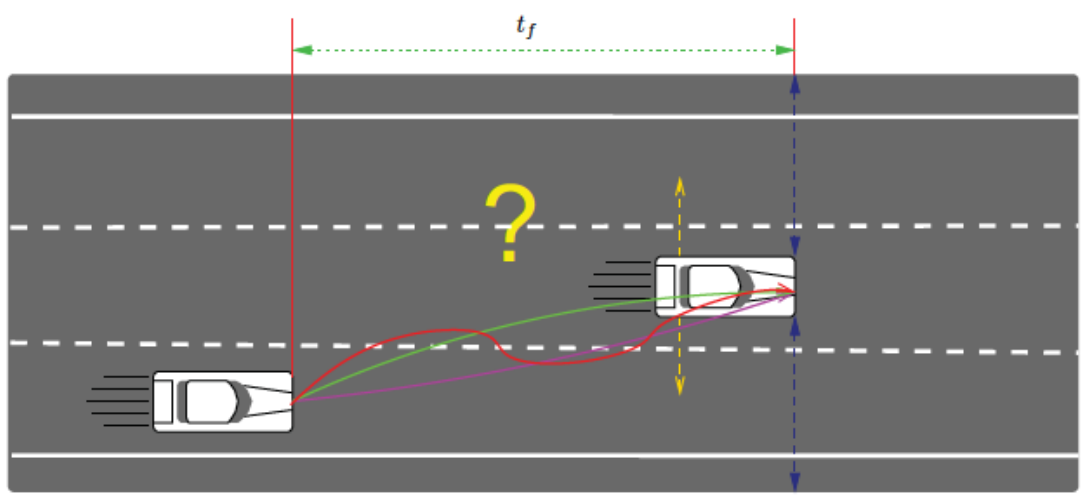

Figure D.1: Scenario considered for the evaluation of Gaussian noise on trajectories

Table D.1: Configuration parameters for the scenario in Appendix D

\begin{tabular}{cc}
\hline Parameter & Value \\
\hline$N$ & 100 \\
$L$ & 1 \\
$M$ & 0 \\
$O$ & 3 \\
$t_{f}$ & $10 \mathrm{~s}$ \\
$v_{I}$ & $33 \mathrm{~m} / \mathrm{s}$ \\
$c v_{I}$ & $5.5432 \mathrm{~m} / \mathrm{s}^{2}$ \\
$W_{l}$ & $6 \mathrm{~m}$ \\
$W_{i}$ & $1 \mathrm{~m}$ \\
$W_{e}$ & $1 \mathrm{~m}$ \\
$W$ & $20 \mathrm{~m}$ \\
$X_{0 j}$ & $5 \mathrm{~m}$ \\
$w_{j}$ & $0 \mathrm{~m}$ \\
$V_{0 j}$ & $0 \mathrm{~m} / \mathrm{s}$ \\
$A_{0 j}$ & $0 \mathrm{~m} / \mathrm{s}^{2}$ \\
$a_{j}^{0}(k)$ & $0 \mathrm{~m} / \mathrm{s}^{2}$ \\
$z_{\beta}$ & $\{0,20\} \mathrm{m}$ \\
$o_{\beta}$ & $\{0,0\} \mathrm{m}^{-2}$ \\
$\theta_{x}$ & $\{1,1\} \mathrm{m}{ }^{-2}$ \\
$\theta_{v}$ & $\{1,0\}(\mathrm{m} / \mathrm{s})^{-2}$ \\
$\theta_{a}$ & $\{0,0\}\left(\mathrm{m}^{-2} / \mathrm{s}^{-3}\right)$ \\
$\sigma_{x}$ & 0.1 \\
$\sigma_{z}$ & $\{0.1,1,5\}$ \\
\hline
\end{tabular}

like the famous Google Car (13). Before discussing in detail the characteristics of the Kalman Filter, we will first express our extended model for the System (D.1) by accounting for the two influencing factors presented earlier:

\section{D.2.1 State variability}

The Kalman Filter model assumes the true state at time $k$ is evolved from the state at $(k-1)$ as expressed by:

$$
\mathbf{x}(k)=\mathbf{F} \cdot \mathbf{x}(k-1)+\mathbf{Y} \cdot \mathbf{u}(k)+\eta(k)
$$

where 


\section{TRAJECTORY OPTIMIZATION UNDER GAUSSIAN NOISE DISTURBANCES}

- $\mathbf{F}$ defines the state transition model applied to the previous state $\mathbf{x}(k-1)$.

- $\mathbf{Y}$ denotes the control-input applied to the controls $\mathbf{u}(k)$.

- $\eta(k)$ is the process noise which is assumed to be drawn from a zero mean multivariate normal distribution with covariance $\mathbf{Q}(k)$ :

$$
\eta(k) \sim N(0, \mathbf{Q}(k))
$$

\section{D.2.2 Measurements variability}

At time $k$ an observation (or measurement) $z(k)$ of the true state $x(k)$ is made according to:

$$
\tilde{\mathbf{x}}(k)=\mathbf{H} \cdot \mathbf{x}(k)+\ell(k)
$$

where $\mathbf{H}$ is the observation model which maps the true state space onto the observed space and $\ell(k)$ is the observation noise which is assumed to be zero mean Gaussian white noise with covariance $\mathbf{R}(k)$ :

$$
\ell(k) \sim N(0, \mathbf{R}(k))
$$

We can observe that in comparison with the initial proposal of System (D.1), we now model our problem as a linear system of equations whose state variables are corrupted by additive Gaussian noise, represented by variable $\eta(k)$ (Gaussian noise process of mean 0 and covariance $\mathbf{Q}(k)$, Eq. (D.5)). Furthermore, we consider that there exists some sort of measurement sensing error, represented by variable $\ell(k)$ (Gaussian noise process of mean 0 and covariance $\mathbf{R}(k)$, Eq. (D.7)).

The position and speed in the extended System (D.4) is described by:

$$
\mathbf{x}(k)=\left[\begin{array}{c}
x \\
\dot{x}
\end{array}\right]
$$

with $\dot{x}$ being the derivative of lateral position respect to time (speed $v(k)$ ) 
Let us now express the $\mathbf{F}, \mathbf{Y}$ and $\mathbf{H}$ matrices by extracting the correspondence of both the controls and state variables with Eqs. (D.5) and (D.7). After some easy operations we get:

$$
\mathbf{F}=\left[\begin{array}{cc}
1 & \Delta t \\
0 & 1
\end{array}\right] \quad \mathbf{Y}=\left[\begin{array}{c}
\Delta t^{2} \\
\Delta t
\end{array}\right] \quad \mathbf{H}=\left[\begin{array}{ll}
1 & 0
\end{array}\right]
$$

Focusing on the covariance matrices regarding process and measurement noise, we get:

$$
\begin{gathered}
\mathbf{Q}=\mathbf{Y} \mathbf{Y}^{\mathrm{T}} \sigma_{x}^{2}=\left[\begin{array}{cc}
\Delta t^{4} & \Delta t^{3} \\
\Delta t^{3} & \Delta t^{2}
\end{array}\right] \sigma_{x}^{2} \\
\mathbf{R}=\mathrm{E}\left[\ell(k) \ell^{T}(k)\right]=\left[\sigma_{z}^{2}\right]
\end{gathered}
$$

From this set of Expressions we are capable now of evaluating how different values of the measurement variance $\sigma_{z}^{2}$ and the state dispersion variance $\sigma_{x}^{2}$ affect the evolution of trajectories ${ }^{1}$. With the Kalman Filter it is possible to minimize the counterproductive effect of both noise processes by using two trajectory regeneration procedures which are enumerated next:

1. Filtering ${ }^{2}$. By means of this process, the Kalman Filter predicts the new values $\mathbf{x}(k)$ of the states taking into account the states history until the instant $(k-1)$.

2. Smoothing. In this second process, the Kalman Filter estimates the new values $\mathbf{x}(k)$ of the states taking into account, apart from the states history until the instant $(k-1)$, the current measurement of the states: $\tilde{\mathbf{x}}(k)$.

We can deduce from the previous two comments that with Smoothing the estimated trajectory will reduce the dispersion respect to Filtering, since we count on more updated information to estimate the current position and speed of the vehicle.

Now it is time to graphically visualize some application examples of the previous concepts. Please, refer to the configuration of parameters in this study, as seen in Table D.1. In the first set of graphs we will show how the trajectory of a vehicle (departing

\footnotetext{
${ }^{1}$ We advise the reader not to get confused here with the functions $\tilde{\sigma}_{\text {dist }}^{2}, \sigma_{\text {dist }}^{2}$ and $\sigma_{m, \text { dist }}^{2}$ from Chapter 6, regarding the distance variance calculation.

${ }^{2}$ Although the term "Kalman Filter" regards all techniques to reduce the influence of noise on the states of a dynamic system, we must not get confused with Filtering, which, as well as Smoothing, describes a specific procedure to fix or reduce trajectory dispersion caused by any noise process.
} 


\section{TRAJECTORY OPTIMIZATION UNDER GAUSSIAN NOISE DISTURBANCES}
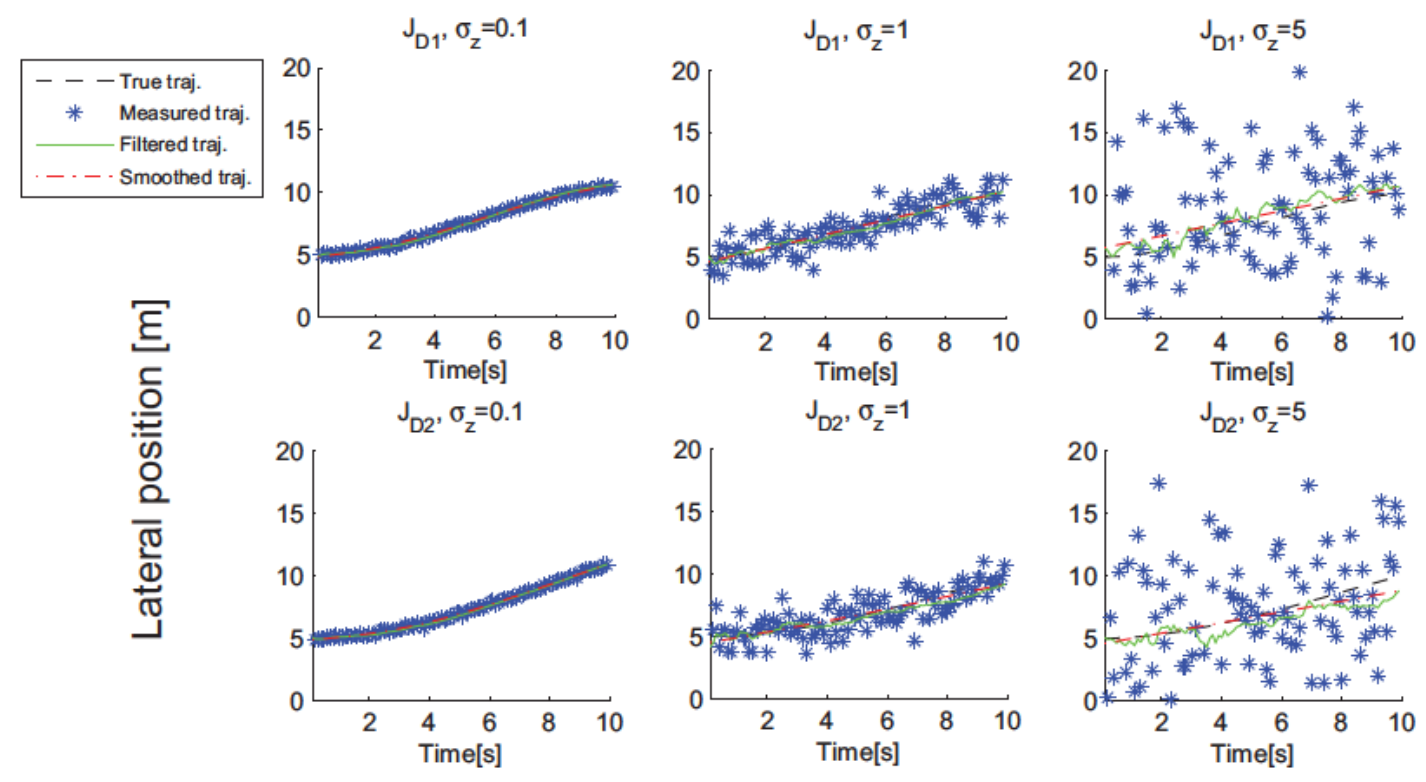

Figure D.2: Kalman Filter effect on trajectories for $J_{D 1}$ and $J_{D 2}$ under measurement noise $\left(\sigma_{z} \in\{0.1,1,5\}\right)$

from a lateral position $x=5 \mathrm{~m}$ and having at most $t_{f}=10 \mathrm{~s}$ to reach the optimum lateral position) is affected by the presence of additive gaussian noise in the states and in the sensor measurements. We will establish a state dispersion variance $\sigma_{x}^{2}=0.1$ for all the examples and will focus only on the influence of noise produced by measurement sensors (measurement variance $\sigma_{z}^{2}$ ). We will include a comparison between the True Trajectory (optimum determined trajectory), the measured trajectory (sensed trajectory while executing the maneuver), and the filtered and smoothed trajectory for the two proposed functionals $J_{D 1}$ and $J_{D 2}$, and for a set of values of the measurement variance $\sigma_{z}^{2}=\{0.1,1,5\}$. If we now draw our attention to Fig. D. 2 we can fairly understand how measurement noise affects the shape of trajectories by producing a dispersion of the optimum path due to the sensed positions along the course (mild dispersion for $\sigma_{z}^{2}=0.1$, medium dispersion for $\sigma_{z}^{2}=1$ and high dispersion for $\sigma_{z}^{2}=5$ ). We can notice that both Filtering and Smoothing reduce the dispersion effect, but Smoothing reduces the still significant variability associated to the filtered trajectory, by using the measurement of the present instant $\tilde{\mathbf{x}}(k)$. Differences between $J_{D 1}$ and $J_{D 2}$, if present, are not clear in Fig. D.2.

Now we turn our attention to the evaluation of the Mean Square Error (MSE) 
between the optimum trajectory, and the measured, filtered and smoothed trajectories for the interval $\sigma_{z}^{2} \in[0,5]$, for both $J_{D 1}$ and $J_{D 2}$. Due to the variability to which the MSE is subject, we have used regression techniques to represent the Degree-2 polynomial which averages the MSE evolution during the evaluated interval. This will make the visualization of graphs easier for comparison purposes.

On the other hand, we will also represent the averaged evolution (with a Degree2 polynomial like with MSE) of the lateral distance respect to the optimum lateral position (which we call LDP) at the last time step $t_{f}$ in order to quantify how different is the final location of the trajectory from the desired position. The associated graph will represent this magnitude for the same interval $\sigma_{z}^{2} \in[0,5]$ as in the last case, and will show the percentage, respect to the total width of the road $W$, of distance far from the optimum lateral position.

If we have now a look at Figs. D.3 and D.4 we will see the evolution of both the MSE and LDP for the functional $J_{D 1}$. For the MSE we can notice that for lower values of the measurement variance $\sigma_{z} \sim 0.1$, sensing noise does not affect the trajectory remarkably, since the measured, filtered and smoothed paths obtain a very low MSE respect to the optimum trajectory. As $\sigma_{z}$ increases it is easily noticeable how necessary it is to use at least Filtering and, if possible, Smoothing in order to correct the path dispersion introduced by noise. Surprisingly, the LDP shows very similar results for both Filtering and Smoothing, since both tend to reach the optimum final position regardless of the evolution of the trajectory. Analyzing now Figs. D.5 and D.6 we can come to the same conclusions as for the $J_{D 1}$ functional. More importantly, we can see that differences between using Filtering and Smoothing for $J_{D 1}$ and $J_{D 2}$ are not as remarkable as to highlight them. This implies that the shape of the traced trajectory does not influence the performance of the Kalman Filter.

\section{D.3 Final remarks}

In this appendix we have carried out a first-step evaluation of how additive Gaussian noise can affect the shape of a single vehicle's trajectory when measurement sensors are affected by such phenomena. We could realize the necessity to use path smoothing techniques such as the Kalman Filter (42), by providing some illustrative results on the performance of this specific tool. In this context, it is the intention of the authors to 


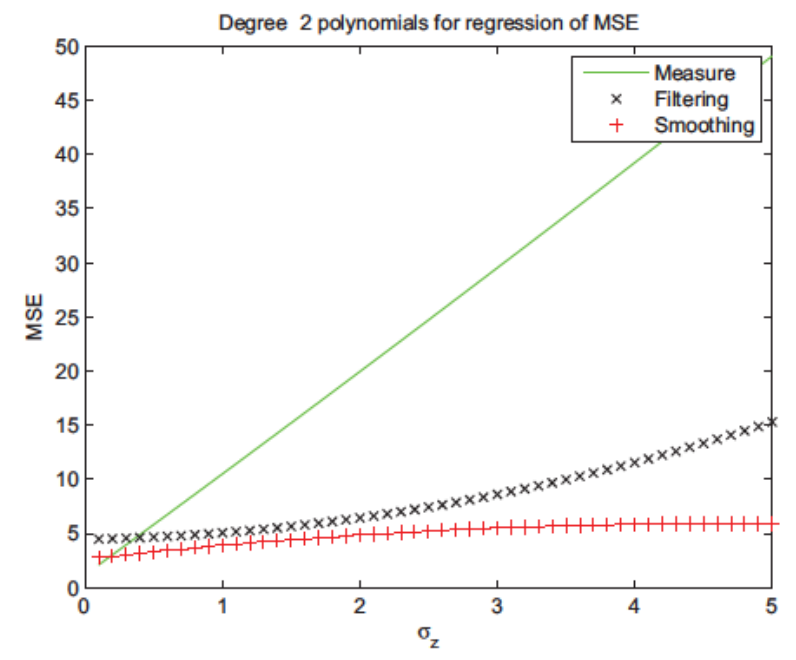

Figure D.3: Degree-2 polynomials for regression of MSE under measurement noise ( $\sigma_{z} \in$ $\{0.1,1,5\})$, for $J_{D 1}$

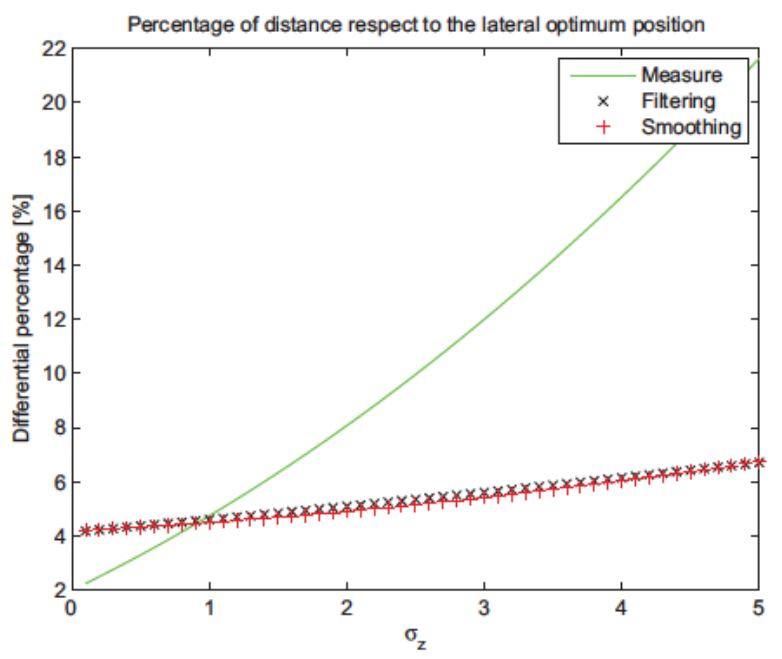

Figure D.4: Percentage of distance respect to the lateral optimum position under measurement noise $\left(\sigma_{z} \in\{0.1,1,5\}\right)$, for $J_{D 1}$

carry out the optimization of trajectories for the general scenario of Chapter 6 where multiple vehicles need to take sudden maneuvering actions in order to avoid one or more obstacles. By introducing noise in the measurement sensors and studying the influence on this sort of critical cooperative actions it will be possible to analyze in detail how vehicles would react to such unpredictable phenomena in realistic situations. 


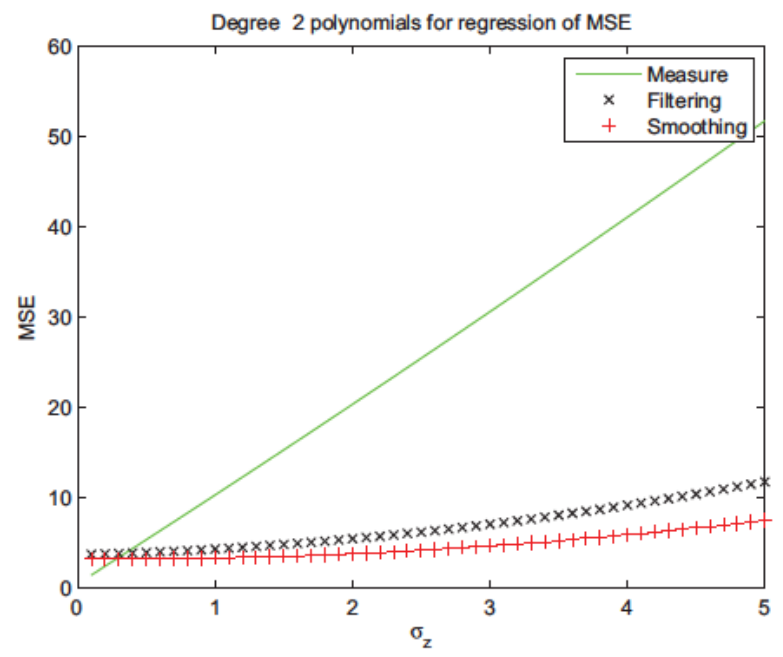

Figure D.5: Degree-2 polynomials for regression of MSE under measurement noise ( $\sigma_{z} \in$ $\{0.1,1,5\})$, for $J_{D 2}$

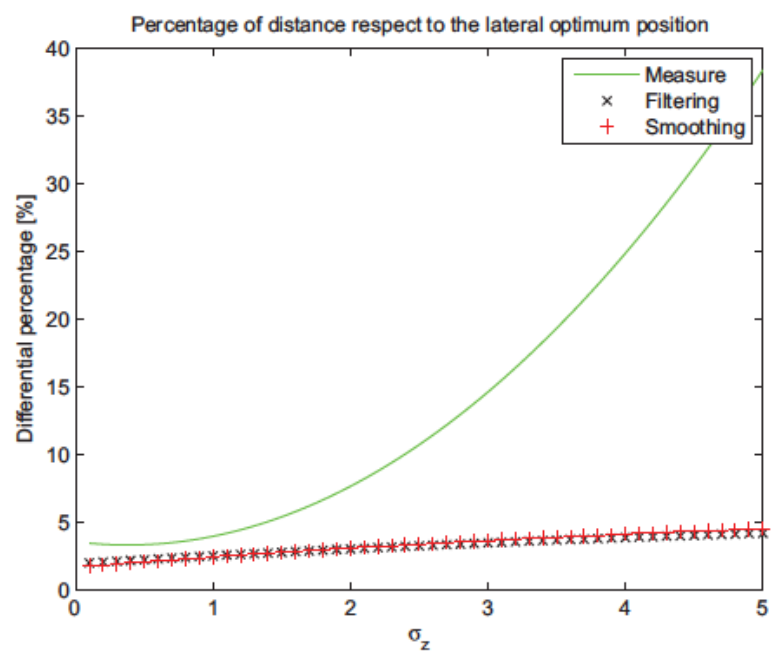

Figure D.6: Percentage of distance respect to the lateral optimum position under measurement noise $\left(\sigma_{z} \in\{0.1,1,5\}\right)$, for $J_{D 2}$ 
D. TRAJECTORY OPTIMIZATION UNDER GAUSSIAN NOISE DISTURBANCES 


\section{References}

[1] International Organization of Motor Vehicle ManufaCTURers. 2010 World Production Statistics. OICA 2010. 3

[2] Specialty Parts. Social Impact of the Car. 2009. 3

[3] R. B. Naumann, A. M. Dellinger, E. Zaloshnja, B. A LaWrence, and T. R. Miller. Incidence and total lifetime costs of motor vehicle-related fatal and nonfatal injury by road user type. Traffic Inj. Prev., 11 (4):353-360, 2010. 4

[4] M. Mscichowski. The Driverless Car. Value Line. 4

[5] M. Williams. PROMETHEUS-The European research programme for optimising the road trans-
port system in Europe. In Driver Information, IEE Colloquium on, pages $1 / 1-1 / 9$, dec 1988. 4, 21

[6] J. Eledath, M. Bansal, G. Kreutzer, A. Das, O. NaroDITSKY, AND W. ENGLish. DARPA Urban Challenge. 2007. 4

[7] J. Alonso RuIz. Maniobras de Conducción Cooperativa entre Vehículos Autónomos. $\mathrm{PhD}$ thesis, Universidad Politecnica de Madrid, Facultad de Informática, Madrid, Spain, 2009. 5, 25

[8] Wireless LAN Medium Access Control (MAC) and Physical Layer (PHY) Specification, 1997. 5, $17,28,33$

[9] J. MARKofF. Google cars drive themselves, in traffic. The New York Times, 10:A1, 2010. 6, 169

[10] H. Fountain. Yes, Driverless Cars Know the Way to San Jose. October 2012. 6

[11] C. Berger. From Autonomous Vehicles to Safer Cars: Selected Challenges for the Software Engineering. Computer Safety, Reliability, and Security, pages $180-189,2012.6$

[12] Technical Infrastructure Google Inc. System Test Engineer, Special Projects, November 2012. 6, 8 170

[13] M. SLOSSON. Google gets first self-driven car license in Nevada. $7,24,175,215$

[14] T. Taleb, K. Ooi, and K. Hashimoto. An Efficient Collision Avoidance Strategy for ITS systems. In Wireless Communications and Networking Conference, 2008. WCNC 2008. IEEE, pages $2212-2217,31$ 2008-april 3 2008. $7,49,63,81$

[15] P. Muhlethaler, A. Laouiti, and Y. Toor. Comparison of Flooding Techniques for Safety Application in VANETs. In Telecommunications, 200\%. ITST' '07. 7 th International Conference on ITS, pages $1-6$, june 2007. 7,49
[16] H. Hartenstein and K. Laberteaux. VANET Vehicular Applications and Inter-Networking Technologies. Intelligent Transport Systems. John Wiley \& Sons, 2009. 7 , $17,18,20,21,25,81,112,169$

[17] S. Biswas, R. Tatchikou, and F. Dion. Vehicle-tovehicle wireless communication protocols for enhancing highway traffic safety Comm. 44(1):74-82, January 2006. 7, 49, 69, 88, 91, 92, 93

[18] A. Kesting. Microscopic Modeling of Human and Automated Driving: Towards Traffic-Adaptive Cruise Control.
Saechsische Landesbibliothek - Staats- und UniversiSaechsische Landesbibliothek - Staats- und
taetsbibliothek Dresden. 7, 64, 104, 108, 115

[19] D.E. KIRK. Optimal Control Theory: An Introduction. Dover books on engineering. Dover, 2004. 7, 133, 154, 155

[20] J.B. Tomas-Gabarron, E. EgEa-Lopez, and J. GarciaHARO. Aplicaciones y futuro de las redes
VANET (I): 802.11p, estándar de comunicaciones inalámbricas para redes vehiculares. Number 410 , pages $20-25$, jul 2009. 10, 173

[21] J.B. Tomas-Gabarron, E. Egea-Lopez, and J. GarciaHaro. Aplicaciones y futuro de las redes VANET (II): Servicios y aplicaciones. Number 411, pages 26-31, oct 2009. 10, 173

[22] J.B. Tomas-Gabarron, E. Egea-Lopez, J. Garcia-Haro, and R. Murcia-Hernandez. Performance Evaluation of a CCA Application for VANETs Using IEEE 802.11p. In Communications Workshops (ICC), 2010 IEEE International Conference on, pages $1-5$, may 2010. 11,174

[23] J.B. Tomas-Gabarron, E. Egea-Lopez, J. Garcia-Haro, AND R.M. HERNANDEZ. Testing viability of relay poli-
cies for reactive CCA applications in VANETs. cies for reactive CCA applications in VANETs. In Wireless and Mobile Computing, Networking and
Communications (WiMob), 2010 IEEE 6th International Conference on, pages 505-512, oct. 2010. 12, 174

24] C. Garcia-Costa, E. Egea-Lopez, J.B. Tomas-Gabarron, J. Garcia-Haro, and Z.J. HaAs. A Stochastic Model for Chain Collisions of Vehicles Equipped With Vehicular Communications. Intelligent Transportation Systems, IEEE Transactions on, 13(2):503-518, june 2012. 12,173

[25] J.B. Tomas-Gabarron, E. EgEa-Lopez, and J. GarciaHARO. Evaluating communications and the IntelHARO. Evaluating communications and the Intelligent Driver Model in a Context of a Chain Col-
lision Avoidance Application for VANETs. Indilision Avoidance Application for VAN
anapolis, IND., USA, sep. 2011. 12, 173

[26] J.B. Tomas-Gabarron, E. Egea-Lopez, and J. GarciaHARO. Optimum maneuvering under time constraints for high speed vehicles. In Wireless and Mobile Computing, Networking and Communication (WiMob), 2012 IEEE 8th International Conference on, oct. $2012.12,173$

27] J.B. Tomas-Gabarron, E. Egea-Lopez, and J. GarciaHARO. Optimization of vehicular trajectories under Gaussian noise disturbances. Future Internet, MDPI, (5):1-20, 2013. 12, 173

[28] J.B. Tomas-Gabarron, E. Egea-Lopez, and J. GarciaHARO. Vehicular trajectory optimization for Cooperative Collision Avoidance at high speeds. IEEE Transactions on Intelligente Transportation Systems, Under review by December 2012. 12, 172

[29] M.H. HeBerT. Intelligent Unmanned Ground Vehicles: Autonomous Navigation Research at Carnegie Mellon. Autonomous Navigation Research at Carnegie Mellon. Kluwe
17,25

[30] S.W. Dellenback. ITS Evolution: Intelligente Vehicles - What is the reality? Southwest Research Institute. 18 
[31] S. Krueger, J. Abele, C. Kerlen, H. Baum, T. Geiler, S. Grawenhoff, J. Schneider, and W.H. Schulz. Exploratory study on the potential socio-economic impact of the introduction of intelligent safety systems in road vehicles. Institute for Transport Economics at the University of Cologne, Tech. Rep. 19

[32] R. O'Toole. Gridlock : why we're stuck in traffic and what to do about it / Randal O'Toole. CATO Institute, Washington, D.C. :, 2009. 19

[33] D.A. Rosen, F.J. Mammano, and R. Favout. An electronic route-guidance system for highway vetronic route-guidance system for highway ve-
hicles. Vehicular Technology, IEEE Transactions on, 19(1): $143-152$, feb 1970. 19

[34] H. KaWASHima. Japanese perspective of driver information systems. Transportation, 17:263-284, 1990. 10.1007/BF02127039. 20

[35] E.D. Dickmanns. An integrated approach to feature based dynamic vision. Computer Vision and Pattern Recognition, 1988. Proceedings CVPR '88., Computer Society Conference on, pages 820-825, 1988. 21

[36] W.J. Gillan. PROMETHEUS and DRIVE: their implications for traffic managers. In Vehicle Navigation and Information Systems Conference, 1989. Conference Record, pages $237-243$, sept. 1989. 21

[37] W. Dabbous and C. Huitema. In PROMETHEUS: Vehicle to Vehicle Communications, Research Report, INRIAto Vehicle Communications, Research Rep
Renault collaboration,, aug 1988. 22

[38] P. Wells and D. Deguire. TAlon: a universal unmanned ground vehicle platform, enabling the mission to be the focus, pages $747-757,2005$, 22

[39] How an autonomous car gets around. The New York Times, oct. 2012. 22, 132

[40] K.E. Olin AND D.Y. TSEnG. Autonomous crosscountry navigation: an integrated perception and planning system. IEEE Expert, 6(4):16 -30, aug. 1991. 22

[41] S. Thrun, W. Burgard, and D. Fox. Probabilistic Robotics (Intelligent Robotics and Autonomous Agents). The MIT Press, 2005. 22, 25

[42] R.E. Kalman. A New Approach to Linear Filtering and Prediction Problems. Journal of Basic Engineering, 82(1):35-45, 1960. 22, 132, 133, 219

[43] H. Hartenstein and K. P. Laberteaux. A tutorial survey on vehicular ad hoc networks. Comm. Mag., 46(6): 164-171, jun 2008. 23,36

[44] J.E. Naranjo, C. Gonzalez, T. de Pedro, R. Garcia, J. Alonso, M.A. Sotelo, and D. Fernandez. AUtopia architecture for automatic driving and maneuvering. In Intelligent Transportation Systems Confervering. In Intelligent Transportation Systems Confer-
ence, 2006. ITSC '06. IEEE, pages $1220-1225$, sept. ence, 2006.

[45] D. M. Lamberson. In An Overview Of The SARTRE Platooning Project, SAE Convergence,, October 2012.

[46] J.B. Tomas-Gabarron, E. Egea-Lopez, and J. GarciaHARO. Evaluación de mecanismos de priorización en 802.11p con VHDL. pages 190-196, sep. 2009. 30,174

[47] Z. Navabi. VHDL: Analysis and Modeling of Digital Systems. McGraw-Hill, Inc., New York, NY, USA, 2nd edition, 1997. 30
[48] IEEE 1609 - Family of Standards for Wireless Access in Vehicular Environments (WAVE), 2011 $33,51,69,91,95,100,169$

[49] S. EICHLer. Performance Evaluation of the IEEE 802.11p WAVE Communication Standard. In Vehicular Technology Conference, 2007. VTC-2007 Fall. 2007 IEEE 66th, pages 2199-2203, 30 2007-oct. 32007. 34,89

[50] C. Campolo, A. Vinel, A. Molinaro, and Y. Koucheryavy. Modeling Broadcasting in IEEE 802.11p/WAVE Vehicular Networks. Communications Letters, IEEE, 15(2): $199-201$, february 2011. 34

[51] M. Torrent-Moreno, D. Jiang, and H. Hartenstein. Broadcast reception rates and effects of priority access in 802.11-based vehicular ad-hoc networks. In Proceedings of the 1st ACM international workshop on Vehicular ad hoc networks, VANET '04, pages 10-18, New York, NY, USA, 2004. ACM. 34, pages $10-18$
$53,81,89,9$

[52] B.S. Gukhool and S. Cherkaoui. IEEE 802.11p modeling in NS-2. In Local Computer Networks, 2008. LCN 2008. 33rd IEEE Conference on, pages $622-626$, oct. 2008. 34

[53] E. Schoch, F. Kargl, M. Weber, and T. Leinmuller Communication patterns in VANETs. Comm. Mag., 46(11):119-125, nov 2008. 35, 36

[54] A.A. CARTER. The status of vehicle-to-vehicle communication as a means of improving crash prevention performance. NHTSA, Tech. Rep, 2005:01 19, 2005. 38

[55] Traffic Sign Recognition, BMW Series 3-Door Retrieved 16/11/2012.39, 169

[56] Mohamed A. Abdel-Aty and A.Essam Radwan. Modeling traffic accident occurrence and involvement. $A c$ cident Analysis and Prevention, 32(5):633 - 642, 2000. 50

[57] J.J. Song, M. Ghosh, S. Miaou, and B. Mallick. Bayesian multivariate spatial models for roadway traffic crash mapping. Journal of Multivariate Analysis, 97(1):246 - 273, 2006. 50

[58] J. Glimm and R.E. Fenton. An accident-severity analysis for a uniform-spacing headway policy. Vehicular Technology, IEEE Transactions on, 29(1):96 - 103, ular Technology, IEEE

[59] K. Taewung and J. Hyun-Yong. Crash Probability and Error Rates for Head-On Collisions Based on Stochastic Analyses. Intelligent Transportation Systems, IEEE Transactions on, 11(4):896 -904, dec 2010. 50,51

[60] A. Touran, M.A. Brackstone, and M. McDonald. A collision model for safety evaluation of autonomous intelligent cruise control. Accident Analysis and Prevention, 31(5):567 - 578, 1999. 50

[61] N. Wisitpongphan, B. Fan, P. Mudalige, V. Sadekar, and O. Tonguz. Routing in Sparse Vehicular Ad Hoc Wireless Networks. Selected Areas in Communications, IEEE Journal on, 25(8):1538 -1556, oct. 2007 $50,65,115$

[62] A. Chakravarthy, S. Kyungyeol, and E. Feron. Preventing Automotive Pileup Crashes in MixedCommunication Environments. Intelligent Transportation Systems, IEEE Transactions on, 10(2):211 225, june 2009. 64

[63] G. Grime And I.S. Jones. Car Collisionsthe Movement of Cars and Their Occupants in Accidents. Proceedings of the Institution of Mechanical Engineers Automobile Division, 184(1):87-136, 1969. 64 
[64] G. Johansson and K. Rumar. Driver's Brake Reaction Times. Human Factors and Ergonomics Society, 13(1):23-27, Feb. 1971. 69

[65] Y. Yang and T.S.P. YUM. Delay distributions of slotted ALOHA and CSMA. Communications, IEEE Transactions on, 51(11):1846 - 1857, nov. 2003. 73

[66] E. Bertolazzi, F. Biral, M. Da Lio, A. Saroldi, and F. TANGO. Supporting Drivers in Keeping Safe Speed and Safe Distance: The SASPENCE Subproject Within the European Framework Programme 6 Integrating Project PReVENT. Intelligent Transportation Systems, IEEE Transactions on, 11(3):525 -538, sept. 2010. 80

[67] J.D. Lee, D.V. McGehee, T.L. Brown, and M.L. Reyes. Collision Warning Timing, Driver Distraction,
and Driver Response to Imminent Rear-End Coland Driver Response to Imminent Rear-End Col-
lisions in a High-Fidelity Driving Simulator. $H u-$ lisions in a High-Fidelity Driving Simulator. Human Factors: The Journal of the Human Factors and
Ergonomics Society, 44(2):314-334, Summer 2002. 80

[68] Y. Kyongsu and J. Chung. Nonlinear brake control for vehicle CW/CA systems. Mechatronics, IEEE/ASME Transactions on, 6(1):17 -25, mar 2001. 80

[69] T. Wada, S. Doi, N. Tsuru, K. Isaji, and H. Kaneko. Characterization of Expert Drivers' Last-Second Braking and Its Application to a Collision Avoidance System. Intelligent Transportation Systems, IEEE Transactions on, 11(2):413-422, june 2010. 80

[70] H.S. TAN and H. Jihua. DGPS-Based Vehicle-toVehicle Cooperative Collision Warning: Engineering Feasibility Viewpoints. Intelligent Transneering Feasibility Viewpoints. Intelligent Trans-
portation Systems, IEEE Transactions on, $\mathbf{7}(4): 415-$ portation Systems,
428 , dec. 2006.81

[71] A.A.K. Jeng, J. Rong-Hong, C. Chien, and C. Tsun-Chieh. Efficient Broadcast Mechanism for Cooperative Collision Avoidance Using Power Control. In Pervasive Systems, Algorithms, and Networks (ISPAN), 2009 10th International Symposium on, pages $266-271$, dec. 2009. 88,89

[72] Y. Fan and S. Biswas. Self-Configuring TDMA Protocols for Enhancing Vehicle Safety With DSRC Based Vehicle-to-Vehicle Communications. Selected Areas in Communications, IEEE Journal on 25(8): $1526-1537$, oct. $2007.88,89$

[73] D. Dhoutaut, A. RÉgis, and F. Spies. Impact of radio propagation models in vehicular ad hoc networks simulations. In Proceedings of the 3rd internaworks simulations. In Proceedings of the 3rd interna'06, pages 40-49, New York, NY, USA, 2006. ACM. 89

[74] V. Desaraju, H.C. Ro, M. Yang, E. Tay, S. Roth, and D. DEL VECCHIO. Partial order techniques for vehicle collision avoidance: Application to an autonomous roundabout test-bed. In Robotics and Automation, 2009. ICRA '09. IEEE International Conference on, pages $82-87$, may 2009. 90

[75] Speed Statistics. TAC (Transport Accident Commission) official webpage, May 2010. 91

[76] A. Kesting, M. Treiber, and D. Helbing. Enhanced intelligent driver model to access the impact of driving strategies on traffic capacity. Royal Society of London Philosophical Transactions Series A, 368:4585-4605, September 2010. 108, 110, 112, 114, $122,124,125$

[77] C. Rudin-Brown and H. Parker. Behavioural adaptation to adaptive cruise control (ACC): implications for preventive strategies. Transportation Research Part F: Traffic Psychology and Behaviour, 7(2):59 $-76,2004.110$
[78] C. Rudin-Brown. 'Intelligent' in-vehicle intelligent transport systems: Limiting behavioural adaptation through adaptive design. Intelligent Transport Systems, IET, 4(4):252-261, december 2010. 110

[79] J. Paunelussen and P.J. Feenstra. Driver Behavior Analysis During ACC Activation and Deactivation in a Real Traffic Environment. Intelligent Transportation Systems, IEEE Transactions on, 11(2):329-338, june 2010. 110, 111

[80] T. Tielert, M. Killat, H. Hartenstein, R. Luz, S. HausBERGER, AND T. BENZ. The impact of traffic-light-tovehicle communication on fuel consumption and
emissions. In Internet of Things (IOT), 2010, pages 1 emissions. In Internet of Thint
$-8,29$ 2010-dec. 1 2010. 111

[81] Nomadic Solutions. Ecogyzer. Retrieved 19/11/2012, 2011. 112

[82] M. Treiber, A. Kesting, and D. Helbing. Delays, inaccuracies and anticipation in microscopic traffic models. Physica A: Statistical Mechanics and its Applications, $\mathbf{3 6 0}(1): 71-88,2006.113$

[83] M. Torrent-Moreno, D. Jiang, and H. Hartenstein. Broadcast reception rates and effects of priority access in 802.11-based vehicular ad-hoc networks. In Proceedings of the 1 st ACM international workshop on Vehicular ad hoc networks, VANET '04, pages 10-18, New York, NY, USA, 2004. ACM. 115

[84] I.M. BERRY. The effects of driving style and vehicle performance on the real-world fuel consumption of U.S. lightduty vehicles. $\mathrm{PhD}$ thesis, Massachusetts Institute of Technology. Dept. of Mechanical Engineering.; Massachusetts Institute of Technology. Technology and Policy Program., Massachusetts, USA, 2010. 116

[85] N. Fatma, L. Christine, and V. Athanasios. Affectively intelligent and adaptive car interfaces. Information Sciences, 180(20):3817 - 3836, 2010. 125

[86] J. Rosen. The Gradient Projection Method for Nonlinear Programming. Part I. Linear Constraints. Journal of the Society for Industrial and Applied Mathematics, 8(1):181-217, 1960. 132, 154

[87] R. Fenton, G. MELociK, And K. Olson. On the steering of automated vehicles: Theory and experiment. of automated vehicles: Theory and experiment.
Automatic Control, IEEE Transactions on, 21(3):306 Automatic Control,
315, jun 1976. 133

[88] R.E. Fenton and I. Selim. On the optimal design of an automotive lateral controller. Vehicular Technology, IEEE Transactions on, $\mathbf{3 7}(2): 108-113$, may 1988 ogy, 133

[89] H. Peng and M. Tomizuka. Optimal Preview Control For Vehicle Lateral Guidance. UC Berkeley: California Partners for Advanced Transit and Highways (PATH) UCB-ITS-PRR-91-16, 1991. 133

[90] Wonshik C. and M. Tomizuka. Lane change maneuver of automobiles for the intelligent vehicle and highway system (IVHS). In American Control Conference, 1994, 3, pages $3586-3587$ vol.3, june-1 july 1994. $133,161,162$

[91] A. Venkatraman and S.P. Bhat. Optimal Planar Turns Under Acceleration Constraints. In Decision and Control, 2006 45th IEEE Conference on, pages $235-240$, dec. 2006. 133

[92] D. Anisi, J. Hamberg, and X. Hu. Nearly time-optimal paths for a ground vehicle. Journal of Control Theory and Applications, 1:2-8, 2003. 133 
[93] L.E. DuBins. On curves of minimal length with a constraint on average curvature and with prescribed initial and terminal positions and tangents. American Journal of Mathematics, 79:497-516, 1957. 133

[94] D.N. Godbole, V. Hagenmeyer, R. Sengupta, and D. SwaROOP. Design of emergency manoeuvres for automated highway system: obstacle avoidance problem. In Decision and Control, 1997., Proceedings of the 36th IEEE Conference on, 5, pages $4774-4779$ vol.5, dec 1997. 133, 134

[95] D. Soudbakhsh, A. Eskandarian, and D. Chichka. Vehicle steering maneuvers with direct trajectory optimization. In Intelligent Vehicles Symposium (IV), 2010 $I E E E$, pages $449-453$, june 2010. 134, 162

[96] N.H. Sledge and K.M. Marshek. Comparison of ideal vehicle lane-change trajectories. In $S A E$ transactions, Society of Automotive Engineers, New York 2010, 106, pages 2024-2027, june 2010. 134

[97] T. SHAMIR. How should an autonomous vehicle overtake a slower moving vehicle: design and analysis of an optimal trajectory. Automatic Control, IEEE Transactions on, 49(4):607-610, april 2004. 134

[98] P. Dingle and L. Guzzella. Optimal emergency maneuvers on highways for passenger vehicles with two- and four-wheel active steering. In American Control Conference (ACC), 2010, pages 5374 -5381, 30 2010-july 2 2010. 134

[99] C. Schmidt, F. Oechisle, and W. Branz. Research on trajectory planning in emergency situations with multiple objects. In Intelligent Transportation Systems Conference, 2006. ITSC' '06. IEEE, pages 988-992, 2006. 134,154

[100] A. Eidehall, J. Pohl, F. Gustafsson, and J. Ekmark. Toward Autonomous Collision Avoidance by Steering. Intelligent Transportation Systems, IEEE Transactions on, 8(1):84-94, march 2007. 134

[101] M. Br annstr om, E. Coelingh, and J. Sjoberg. ModelBased Threat Assessment for Avoiding Arbitrary
Vehicle Collisions. Intelligent Transportation Systems, Vehicle Collisions. Intelligent Transportation Systems,
IEEE Transactions on, 11(3):658 -669, sept. 2010. 135

[102] R.P. Stanley and G.C. Rota. Enumerative Combinatorics: Cambridge studies in advanced mathematics. torics: Cambridge studies in advanced math
Cambridge University Press, 2000. 145, 146

[103] S. Boyd and L. Vandenberghe. Convex Optimization. Cambridge University Press, New York, NY, USA, 2004. 154
[104] G. Reymond, A. Kemeny, J. Droulez, and A. Berthoz. Role of Lateral Acceleration in Curve Driving: Driver Model and Experiments on a Real Vehicle and a Driving Simulator. Human Factors: The Journal of the Human Factors and Ergonomics Society, 43(3):483-495, Fall 2001. 156

105] D.G. de Carreteras. Centro de publicaciones. MinisteRIO DE FOMENTO. Trazado. Instrucción de Carreteras. Norma 3.1-IC., 2011. 157, 187

[106] V. A. Herndon. Reaffirmed Mission for Autonomous Audi TTS Pikes Peak. 169

[107] V. Vijayenthiran. 2013 Mercedes-Benz S-Class To Debut Autonomous Driving System. 169

[108] J.B. Tomas-Gabarron and J. Garcia-Haro. Protocolo MAC basado en identificadores para redes Adhoc vehiculares. In Telecoforum 2008, March 2009. 173

[109] R. Murcia-Hernandez, C. Garcia-Costa, J.B. TomasGabarron, E. Egea-Lopez, and J. Garcia-Haro. Parallelization of a mathematical model to evaluate a CCA application for VANETs. In 1st International Conference on Simulation and Modeling Methodologies, Technologies and Applications (SIMULTECH), July 2011. 174

[110] C. Garcia-Costa, J.B. Tomas-Gabarron, E. Egea-Lopez, AND J. Garcia-Haro. Speeding Up the Evaluation of J. GARClA-HARO. Speeding Up the Evaluation of a Mathematical Model for VANETs Using OpenMP. In NUNO PINA, JANUSZ KACPRZYK, AND JOAQUIM FILIPE, editors, Simulation and Modeling Methodologies,
Technologies and Applications, $\mathbf{1 9 7}$ of Advances in InTechnologies and Applications, 197 of Advances in In-
telligent Systems and Computing, pages 23-37. Springer telligent Systems and Comput
Berlin Heidelberg, 2013. 174

111] IEEE Standard for Information TechnologyPortable Operating System Interface (POSIX)Part 1: System Application Program Interface (API)- Amendment D: Additional Real time Extensions [C Language], 1999. 178

112] W. Shie-Yuan and L. Chin-Che. NCTUns 5.0: A Network Simulator for IEEE 802.11(p) and 1609 Wireless Vehicular Network Researches. In Vehicular Technology Conference, 2008. VTC 2008-Fall. IEEE 68 th, pages $1-2$, sept. 2008. 178,179

113] S.Y. WANG AND C.L. CHOU, NCTUns tool for wireless vehicular communication network researches.
Simulation Modelling Practice and Theory, 17(7):1211 Simulation Modellin

[114] R.L. Stratonovich. Topics in the Theory of Random Noise: Peaks of random functions and the effect of noise on relays, nonlinear self-excited oscillations in the presence of noise. Mathematics and Its Applications Series. CRC PressINC, 1967. 213 


\section{Declaration}

I herewith declare that I have produced this Work without the prohibited assistance of third parties and without making use of aids other than those specified; notions taken over directly or indirectly from other sources have been identified as such. This Work has not previously been presented in identical or similar form to any other Spanish or foreign examination board. The thesis work was conducted under the supervision of Joan García Haro at Universidad Politécnica de Cartagena.

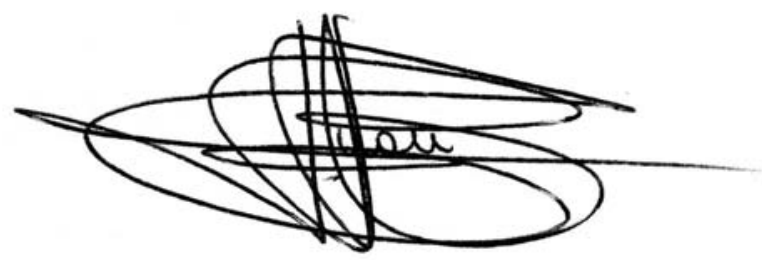

Juan Bautista Tomás Gabarrón

March 2013, Cartagena, SPAIN 